C-A/AP/\#228

February 2006

\title{
Electron Clouds in the Relativistic Heavy Ion Collider
}

Ubaldo Iriso Ariz (University of Barcelona)

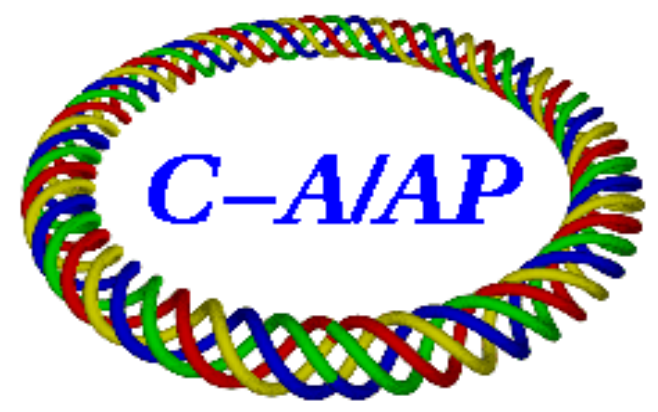

Collider-Accelerator Department Brookhaven National Laboratory Upton, NY 11973 
UNIVERSITAT DE BARCELONA

B

\section{ELECTRON CLOUDS IN THE RELATIVISTIC HEAVY ION COLLIDER}

Ubaldo Iriso Ariz

2005 



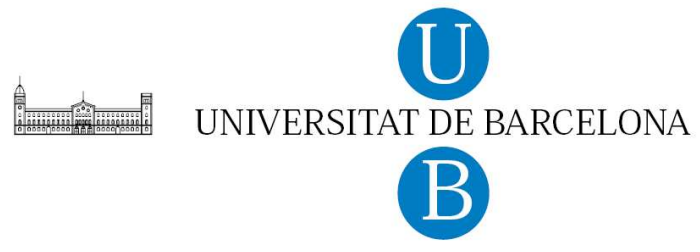

\section{DEPARTAMENT DE FÍSICA APLICADA I ÒPTICA UNIVERSITAT DE BARCELONA \\ Avda. Diagonal 647 \\ E-08028, Barcelona}

Programa de Doctorat: Tècniques Instrumentals de la Física i la Ciència de Materials Bienni: 2000-2002

Director: Prof. Steve Peggs

Co-Director: Prof. Joan Esteve i Pujol

Títol: Electron Clouds in the Relativistic Heavy Ion Collider

Autor: Ubaldo Iriso Ariz

Memòria presentada per a l'obtenció del grau de Doctor

Barcelona, Novembre del 2005 
The work in this thesis has been performed in the Collider-Accelerator Department of the Brookhaven National Laboratory in Upton, New York - 11973 (USA), under the direction of:

Dr. Steve Peggs, Leader for the LHC Accelerator Research Program at Brookhaven National Laboratory and Adjunct Professor in the Physics Department of Stony Brook University (Stony Brook, New York - 11794, USA),

and

Dr. Joan Esteve i Pujol, Professor in the Applied Physics and Optics Department in the University of Barcelona (Avda. Diagonal 645, Barcelona, Spain). 


\section{Abstract}

Inside a radiofrequency cavity, the electric field accelerates electrons (produced by field emission, photo-emission, residual gas ionization, etc) towards the system's chamber wall. Depending on the wall's surface properties and on the electron impacting energy, these primary electrons produce secondary electrons when they hit the chamber wall, which in turn are accelerated if the electric field is at the correct phase. This bouncing back and forth between surfaces is called the electron multipacting, or multipactoring, effect. It was first described by Farnsworth in the 1930's [1]. The name is derived from the term "multiple electron impacts". If the number of emitted electrons per impinging electron, given by the Secondary Electron Yield (SEY) of the wall surface, is greater than unity, the electron density inside the vacuum chamber increases exponentially creating a so-called electron cloud. This usually ends in catastrophic drop in system performance. Depending on the multipacting scenario, the exponential growth is finally limited by the available power and/or space-charge effects.

Inside an accelerator's vacuum chamber, or beam pipe, the radiofrequency electric field is provided by the beam, and the effect is often referred to as Beam Induced Multipacting (BIM). In this case, the electron cloud is defined as an accumulation of electrons inside the beam pipe which, if sufficiently strong, can affect the machine performance by increasing the vacuum pressure, producing beam instabilities, causing beam loss and/or interference in beam diagnostics [2]. The proton storage ring of the Budker Institute of Nuclear Physics (BINP, Novosibirsk) in 1965 [3] and the Ion Storage Ring at CERN in 1972 [4] are perhaps the first machines to suffer from electron clouds. In the 1990's, electron clouds were observed in many accelerators with positively charged particles (the PSR at LANL, the PF at KEK, the PEP-II at SLAC, the SPS at CERN, etc), often acting as a fundamental limit to machine performance resulting from the aforementioned effects. These limitations led accelerator scientists to develop complex computer simulation codes that model the circumstances in which the build up of an electron cloud occurs [5]. The phenomenon is quite sensitive to a host of accelerator parameters including beam bunch intensity, beam bunch spacing, beam dimensions, chamber geometry, and properties of the chamber surface material. If a given threshold is crossed, an electron cloud quickly develops or disappears. The least well known property is the SEY dependence on electron energy, especially for low energy electrons [6]. It plays a crucial role because it determines the number of surviving electrons when the electric field is absent, a condition that occurs regularly during the gap between the passage of two consecutive beam bunches.

In 2001, after one year of operation, the first attempts to fill the Relativistic Heavy Ion Collider (RHIC) at BNL with intense ion beams resulted in intolerable pressure rises inside the vacuum chamber. These unexpected pressure rises limited machine performance to such an extent that design intensities for RHIC ion beams could not be reached [7]. Since RHIC was not equipped with specialized instrumentation for electron cloud detection, any conclusions about their role in these pressure rises were drawn indirectly from beam analysis 
and from the results of computer simulation codes [8]. Therefore, a first priority of this thesis research was to install electron detectors around the RHIC ring, and thus obtain reliable diagnostics for the phenomenon. With these diagnostics, one can further study different mechanisms that might be used to mitigate the multipacting effect. These electron detectors will also provide experimental data from a real machine that can be used to benchmark the results of computer simulation codes.

In a novel approach, this thesis also shows how electron cloud evolution can be modeled by means of simple non-linear maps of the form $\rho_{\mathrm{m}+1}=f\left(\rho_{m}\right)$, where $\rho_{m}$ denotes the electron density inside the vacuum chamber after the passage of beam bunch $m$. This approach leads to an improved intuitive understanding of the evolution of electron clouds as well as a reduction in simulation computational time by seven orders of magnitude. To first order, the function $f\left(\rho_{m}\right)$ is expressed by $\rho_{\mathrm{m}+1}=a \rho_{m}$, where $a$ is the bunch to bunch electron multiplication gain, and $a=1$ sets the threshold for the build up or mitigation of an electron cloud. An analytical expression for $a$ involving the different parameters therefore provides the region in parameter space where RHIC can be reliably operated avoiding the harmful effects of the electron cloud. 


\section{Contents}

Abstract $\quad$ v

1 Introduction $\quad 3$

1.1 The Relativistic Heavy Ion Collider, RHIC . . . . . . . . . . . . . . . 3

1.2 Linear beam dynamics . . . . . . . . . . . . . . . . . . . 5

1.2.1 Magnetic fields and transverse particle motion . . . . . . . . . . 7

1.2.2 Electric fields and longitudinal particle motion . . . . . . . . . . . . 8

1.3 Consequences of a pressure rise . . . . . . . . . . . . . . . . . . . . . . . . . . . . . .

1.3.1 Beam lifetime . . . . . . . . . . . . . . . . 11

1.3.2 Experimental backgrounds . . . . . . . . . . . . . . . . . . . . . . . . . . . . . . .

1.3.3 Emittance growth . . . . . . . . . . . . . . . . 12

2 Electron cloud effect in accelerators $\quad 15$

2.1 Introduction . . . . . . . . . . . . . . . . . . . . . . 15

2.2 Single-bunch regime . . . . . . . . . . . . . . . . . . . . . . . . . . . . . . . . . . .

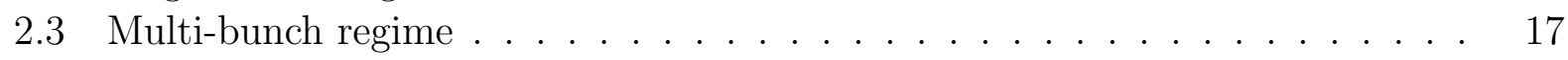

2.4 Electron Cloud at RHIC . . . . . . . . . . . . . . . . . . . . . 18

2.5 Pressure rises in RHIC during Run-2 . . . . . . . . . . . . . . . . 19

3 Electron cloud build-up mechanism $\quad 23$

3.1 Primary electron sources . . . . . . . . . . . . . . . . . . 23

3.2 The electron-bunch interaction . . . . . . . . . . . . . . . . . 24

3.2.1 Cylindrical bunch . . . . . . . . . . . . . . . . . . . . . . . . . . . . . . . . . . 25

3.2.2 Non-uniform bunch charge distribution . . . . . . . . . . . . . . 26

3.3 Secondary electron yield . . . . . . . . . . . . . . . . . . . . . . 28

3.4 Saturation effects . . . . . . . . . . . . . . . . . . . . . . . . . . . . . . . . 32

3.5 Electron cloud computer simulation codes . . . . . . . . . . . . 33

3.5.1 Cylindrical Symmetric Electron Cloud, CSEC . . . . . . . . . . . . 33

3.5.2 Example for RHIC . . . . . . . . . . . . . . . . . . . . . . 34

3.5.3 Average energy at wall impact . . . . . . . . . . . . . 36

4 The RHIC electron detector $\quad 39$

4.1 A Retarding Field Analyzer . . . . . . . . . . . . . . . . . . . . . . . . 39

4.2 Data acquisition and control system . . . . . . . . . . . . . 40 
4.3 Multipacting signal in the electron detector . . . . . . . . . . . . . . . . 41

4.3.1 MADC data acquisition system . . . . . . . . . . . . . . . . . 42

4.4 Image currents in the electron detector . . . . . . . . . . . . . . . . . 43

4.5 Experimental examples . . . . . . . . . . . . . . . . . . . . . . . . . . . . . . . . . . . . 45

4.6 Calibration of the electron detector . . . . . . . . . . . . . . . . 47

4.6.1 Frequency calibration . . . . . . . . . . . . . . 47

4.6 .2 Electron flux to the wall . . . . . . . . . . . . . . . . 48

5 Electron induced molecular desorption in the RHIC beam pipes 51

5.1 Pressure rise due to an electron cloud . . . . . . . . . . . . . . . . 51

5.2 Experimental set up . . . . . . . . . . . . . . . . . 53

5.2.1 Unbaked surface instrumentation . . . . . . . . . . . . . 53

5.2 .2 Baked surface instrumentation . . . . . . . . . . . . . . . 55

5.3 Electron induced desorption of unbaked stainless steel . . . . . . . . . . . . 56

5.4 Electron induced desorption of baked stainless steel . . . . . . . . . . . . . 58

5.5 Electron energy during multipacting conditions . . . . . . . . . . . . . . 60

5.5.1 Experimental results using the electron detector . . . . . . . . . . . 60

5.5.2 Simulation results using CSEC . . . . . . . . . . . . . . . . 61

5.6 Summary . . . . . . . . . . . . . . . . . . . 63

6 Electron clouds during beam injection $\quad 65$

6.1 Introduction . . . . . . . . . . . . . . . . . . 65

6.2 Electron flux and pressure evolution during beam injection . . . . . . . . 66

6.3 Experimental data . . . . . . . . . . . . . . . . . . . 67

6.4 Benchmarking simulations with experimental data . . . . . . . . . . . 70

6.4.1 Experimental values . . . . . . . . . . . . . 70

6.4.2 Simulation results . . . . . . . . . . . . . . . . . 72

$7 \begin{array}{ll}\text { Pressure rise in the experimental regions } & 75\end{array}$

7.1 Two beams in a common beam pipe . . . . . . . . . . . . . . 76

7.2 Experimental results . . . . . . . . . . . . . . . . . . . . . . 78

7.2.1 Electron cloud decay in the interaction regions . . . . . . . . . . . . . 79

7.3 Bunch length influence . . . . . . . . . . . . . . . . . . . 80

7.4 Transition pressure rise . . . . . . . . . . . . . . . . . 81

7.4.1 Transition pressure rise due to electron clouds . . . . . . . . . . . . 82

7.4.2 Beam loss induced desorption . . . . . . . . . . . . . . . 87

8 Possible remedies $\quad 89$

8.1 Bunch pattern . . . . . . . . . . . . . . . . . . . . . . 89

8.1.1 Operational experience using different bunch patterns . . . . . . . . . 90

8.2 Solenoid fields . . . . . . . . . . . . . . . . . . . . . . . 91

8.2.1 Electron motion in the presence of a solenoid field . . . . . . . . . . . 93

8.2.2 Experimental results . . . . . . . . . . . . . . . . . . 94

8.3 Non Evaporable Getter coatings . . . . . . . . . . . . . . . . . . 96 
8.3.1 Motivation for a getter film . . . . . . . . . . . . . . 96

8.3.2 Evaluation of the NEG coating installed at RHIC . . . . . . . . . . . 98

8.4 Future strategies . . . . . . . . . . . . . . . . . . . . . . 99

9 Maps for electron clouds $\quad 103$

9.1 Motivation ........................... 103

9.2 The bunch to bunch evolution . . . . . . . . . . . . . . . . 105

9.3 The map candidates . . . . . . . . . . . . . . . . . . . . . . . . . . . 108

9.4 Minimization of electron density at RHIC . . . . . . . . . . . . . . 110

9.4.1 Simulations for different bunch patterns . . . . . . . . . . . . 112

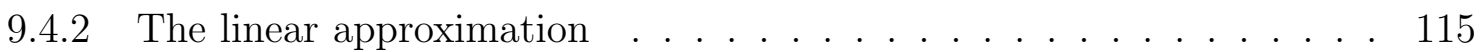

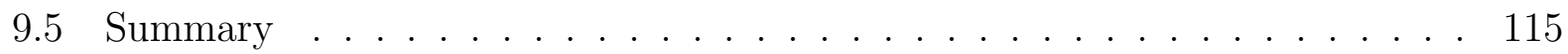

10 The linear map coefficient $\quad 117$

10.1 Introduction . . . . . . . . . . . . . . . . . . . . . . . . . 117

10.1.1 Energy gain and first electron-wall collision . . . . . . . . . . . 118

10.1.2 High energy electron-wall collisions . . . . . . . . . . . . . . . . 120

10.1.3 Low energy electron-wall collisions . . . . . . . . . . . . . . . . 121

10.1.4 Total survival of low and high energy electron-wall collisions . . . . . 122

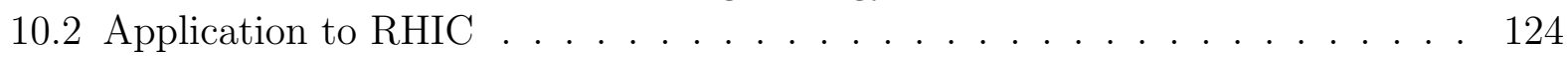

10.3 Comparison with simulation codes . . . . . . . . . . . . . 127

11 Maps for coupled electron and ion clouds 131

11.1 Introduction . . . . . . . . . . . . . . . . . . . . . . . . 131

11.1.1 A vacuum instability . . . . . . . . . . . . . . . . . 131

11.1.2 First and second order phase transitions in RHIC . . . . . . . . . . . 132

11.2 Phase transitions using Maps for Electron Clouds . . . . . . . . . . . . . . 133

11.3 Ion clouds . . . . . . . . . . . . . . . . . . . . . . . . . . . . . . . . . . . . . . . . . . . . . . . . . .

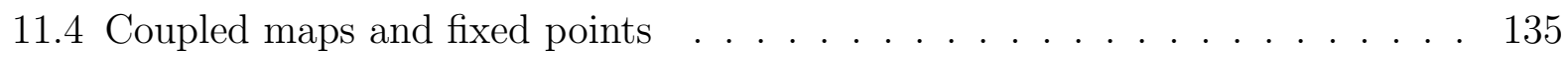

11.4.1 Stability condition . . . . . . . . . . . . . . . 135

11.5 A simple coupled maps model . . . . . . . . . . . . . . . . . . . . . . . . . . . . . . . . . . . . . . . . . . . . .

11.5.1 Numerical application . . . . . . . . . . . . . . . . . . 137

11.6 First order phase transitions and hysteresis . . . . . . . . . . . . . . 139

11.7 Additional dynamical phases . . . . . . . . . . . . . . . . . . . 141

11.8 Discussion . . . . . . . . . . . . . . . . . . . . . . . . . . . . . . . . . 141

12 Conclusions $\quad 145$

$\begin{array}{lr}\text { A Cut-off frequency in a high pass filter } & 149\end{array}$

B Decay of an electron cloud $\quad 151$

C Materials and geometry of the RHIC interaction regions 153 
D Linear motion convergence near a fixed point $\quad 155$

D.1 Elliptical motion . . . . . . . . . . . . . . . . . . . . . . 156

D.2 Hyperbolic motion . . . . . . . . . . . . . . . . . 156

D.3 Summary . . . . . . . . . . . . . . . . . . 157

$\begin{array}{lr}\text { Bibliography } & 158\end{array}$

$\begin{array}{lr}\text { Publications } & 167\end{array}$

$\begin{array}{lr}\text { Acknowledgments } & 169\end{array}$ 


\section{PART I}

\section{INTRODUCTION}





\section{Chapter 1}

\section{Introduction}

\subsection{The Relativistic Heavy Ion Collider, RHIC}

RHIC is a $3.833 \mathrm{~km}$ circumference collider consisting of two superconducting rings that intersect at six interaction regions. The two rings are referred to as "blue" and "yellow". The beam circulates in the clockwise direction in the blue ring and counterclockwise in the yellow ring. RHIC started its Physics operation in 2000, and it is capable of accelerating a variety of species from protons to fully stripped gold or copper ions. The maximum beam energy is $100 \mathrm{GeV} / \mathrm{u}$ for gold and $250 \mathrm{GeV}$ for protons [7]. A sketch of the BNL accelerator complex, showing the RHIC injectors, low energy beam-lines, and location of the interaction regions is shown in Fig. 1.1.

RHIC serves five experiments, STAR, PHENIX, BRAHMS, PHOBOS, and PP2PP. The Solenoidal Tracker at RHIC (STAR) is one of the two large detectors at RHIC and is located in the Interaction Point at 6 o'clock, IP6 - see Fig. 1.1. It is specialized in tracking the thousands of particles produced by each ion collision, and its goal is to obtain a fundamental understanding of the structure of interactions between hadrons [9]. The Pioneering High Energy Nuclear Interaction eXperiment (PHENIX) detector is the other large detector, located in IP8. It is designed specifically to measure direct probes of the collisions such as leptons and photons [10]. The PHOBOS experiment is one of two "small" detectors and is located in the IP10 position of RHIC. It is designed to examine and analyze a very large number of unselected collisions with the premise that rare events will be readily identified [11]. The Broad Range HAdron Magnetic Spectrometer (BRAHMS) is the other small detector at RHIC located at IP2. It provides precise measurements of charged hadrons over a large range of rapidity a transverse momentum [12]. The PP2PP experiment shares the IP2 interaction region with BRAHMS. Its goal is to study proton-proton elastic scattering and therefore participates only in proton runs [13].

The first physics run was during the year 2000 with gold beams. Subsequent runs have taken advantage of the flexibility of RHIC by colliding gold, deuteron, proton, and copper beams, even combining two different species in each ring. For instance, Run-3 used deuteron beams in the blue ring with gold in the yellow ring. This is summarized in Table 1.1.

One figure of merit for a collider is the Luminosity. Suppose a round bunch of $N_{b}$ particles 


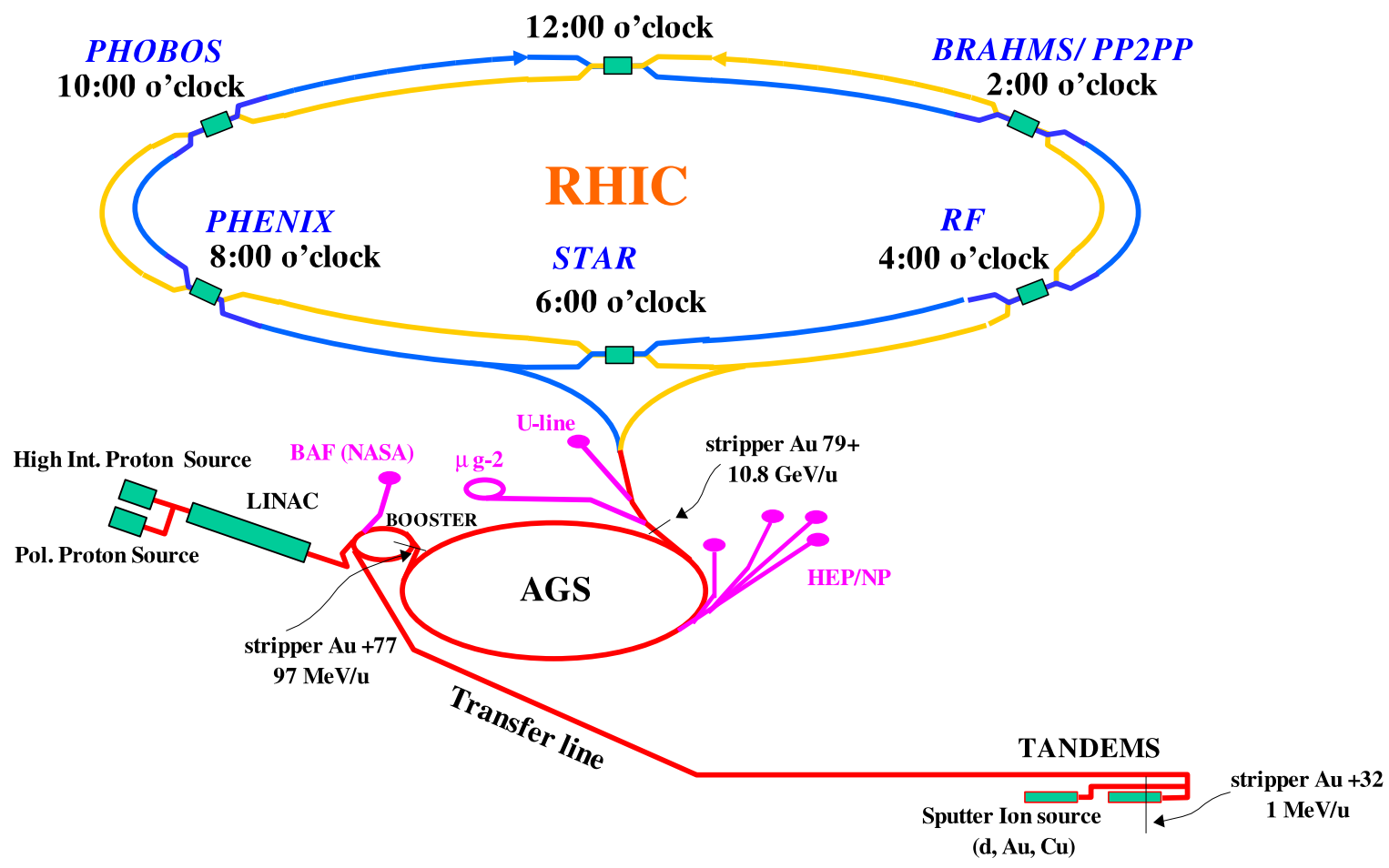

Figure 1.1: Sketch of the hadron accelerator complex at Brookhaven National Laboratory. The path of a Gold ion from its creation until its injection into RHIC is shown.

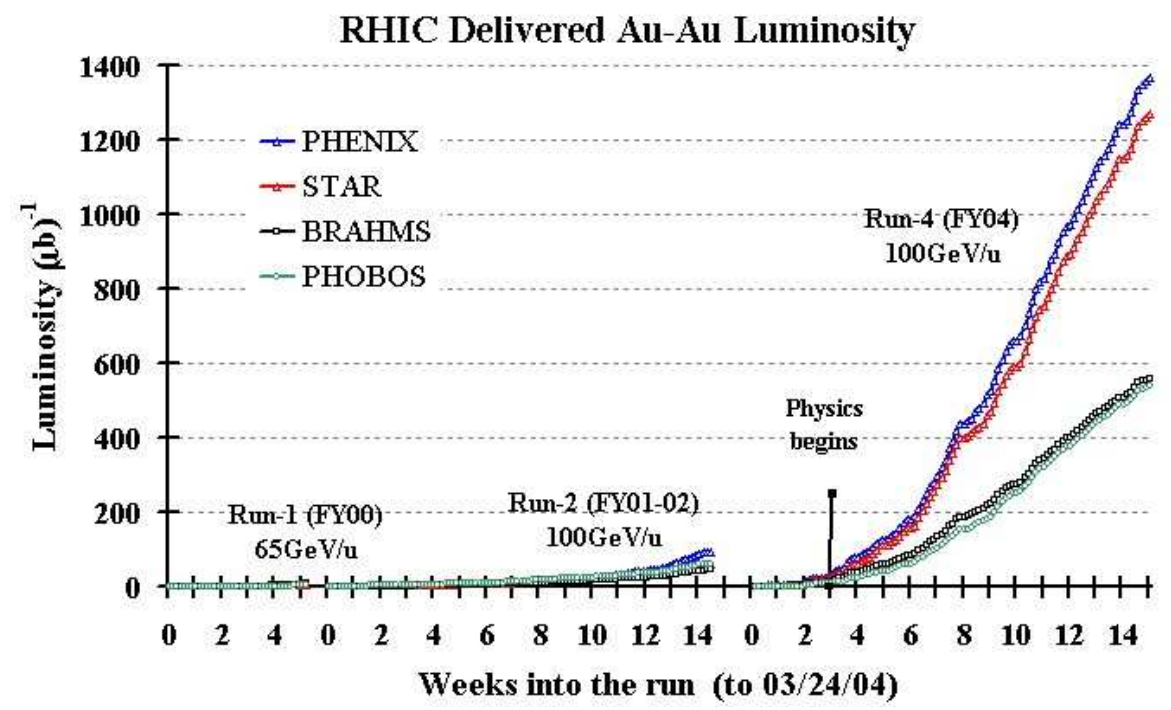

Figure 1.2: Integrated luminosity evolution for the different runs using gold ions in both RHIC rings. Courtesy of W. Fischer. 
Table 1.1: Summary of RHIC parameters during the physics runs to date. By request of the experiments, during Runs 4 and 5 and for a short period ( $\approx 10$ days), the beams were accelerated only up to $31.2 \mathrm{GeV} / \mathrm{u}$. The shown integrated luminosity was delivered to one of the high-luminosity experiments, PHENIX.

\begin{tabular}{|c|c|c|c|c|}
\hline & year & specie(s) & energy $[\mathrm{GeV} / \mathbf{u}]$ & $\begin{array}{c}\text { integrated } \\
\text { luminosity }\end{array}$ \\
\hline Run 1 & 2000 & gold & 65 & $7.3 \mu \mathrm{b}^{-1}$ \\
\hline \multirow{2}{*}{ Run 2 } & 2001 & gold & 100 & $93 \mu \mathrm{b}^{-1}$ \\
& $2001 / 2002$ & proton & 100 & $0.35 \mathrm{pb}^{-1}$ \\
\hline \multirow{2}{*}{ Run 3 } & $2002 / 2003$ & deuteron - gold & 100 & $24.2 \mathrm{nb}^{-1}$ \\
& 2003 & proton & 100 & $2.0 \mathrm{pb}^{-1}$ \\
\hline \multirow{2}{*}{ Run 4 } & $2003 / 2004$ & gold & 100 & $1370 \mu \mathrm{b}^{-1}$ \\
& 2004 & proton & 100 & $3.0 \mathrm{pb}^{-1}$ \\
\hline \multirow{2}{*}{ Run 5 } & $2004 / 2005$ & copper & 100 & $15 \mathrm{nb}^{-1}$ \\
& 2005 & proton & 191.5 & $12.6 \mathrm{pb}^{-1}$ \\
\hline
\end{tabular}

collides head-on with a bunch of identical intensity moving in the opposite direction. The luminosity, $L$ is defined by [14]

$$
L=n \frac{N_{b}^{2}}{\tau_{r} 4 \pi \sigma_{t}^{2}},
$$

where $n$ is the number of colliding bunches per beam, $\tau_{r}$ is the revolution period, and $\sigma_{t}$ is the transverse rms radius of the bunch of particles. Often a more informative way to characterize the collider performance is luminosity integrated over time, expressed in units of inverse barns*. Table 1.1 lists the integrated luminosity during each run to date. Different species have different achievable bunch intensities and sizes. Therefore, the integrated luminosity for gold-gold collisions (Run-2 and Run-4) is expressed in units of $\left[\mu \mathrm{b}^{-1}\right]$, while it is expressed in units of $\left[\mathrm{pb}^{-1}\right]$ for proton-proton collisions. A "historical" evolution of this magnitude during the different runs colliding gold ions is depicted in Fig. 1.2.

\subsection{Linear beam dynamics}

A detailed introduction to accelerator physics can be found in References [14-16], whence the treatment in this work is based. The motion of a particle of charge $q$ in an accelerator is determined by the relativistic extensions to Newton's laws and the Lorentz force,

$$
\frac{d \vec{p}}{d t}=q(\vec{E}+\vec{v} \times \vec{B})
$$

where $\vec{v}$ is the particle velocity, $\vec{E}$ is the electric field (used mainly for particle acceleration and longitudinal confinement), and $\vec{B}$ is the magnetic field (used mainly to steer and focus

$* 1 \mathrm{~b}=1$ barn $=10^{-24} \mathrm{~cm}^{2}$ 


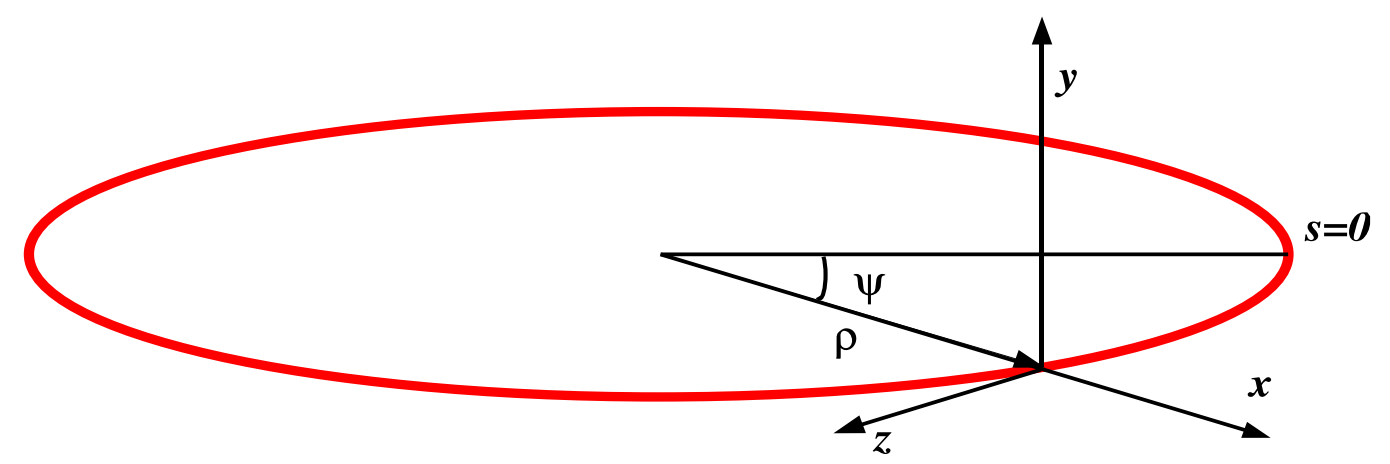

Figure 1.3: Frenet-Serret coordinate system along the design orbit.

the particle motion). The relativistic particle momentum, $\vec{p}$ is expressed as

$$
\vec{p}=\gamma m \vec{\beta} c
$$

where $m$ is the rest mass, $c$ is the speed of light, and $\vec{\beta}$ and $\gamma$ are the relativistic velocity and beam Lorentz factor, respectively, defined as

$$
\begin{aligned}
& \vec{\beta}=\vec{v} / c \\
& \gamma=\frac{1}{\sqrt{1-\beta^{2}}} .
\end{aligned}
$$

To describe the particle's motion, it is useful to define a reference system as shown in Fig. 1.3 based on the design orbit in an ideal circular accelerator of radius $\rho$. The position of a particle may be parameterized in terms of an angle $\psi$, so that the arc length to a different point would be then $\Delta s=\rho \Delta \psi$. The local cartesian coordinate system described in Fig. 1.3 moving along with the beam particle is convenient for parameterization of the motion of a particle traveling on some trajectory slightly different from the design orbit. In this local system, the $z$-axis points in the direction of motion, the $y$-axis points up, and the $x$-axis points radially outward from the center of the circle.

The local radius of the design orbit is determined by the vertical dipole field, $B$ and the particle momentum according to

$$
\rho=\frac{p}{q B},
$$

where $q$ is the particle charge. This relation is used to define the magnetic rigidity as $B \rho=p / q$, or the ratio of momentum to charge. It is written as a single symbol, $(B \rho)$, and for a particle with charge $q=Z|e|$ and atomic mass $A$, it is conventionally calculated using the relation

$$
B \rho \quad[\mathrm{T} \mathrm{m}]=3.33 p \quad[\mathrm{GeV} / \mathrm{c}] A / Z,
$$

in units of $[\mathrm{T} \mathrm{m}]$ when $p$ is expressed in $[\mathrm{GeV} / \mathrm{c}]$. 


\subsubsection{Magnetic fields and transverse particle motion}

The magnetic fields in an accelerator can be expanded in terms of field multipoles. To first order, these fields are:

$$
\begin{aligned}
& B_{x}=\frac{\partial B_{y}}{\partial x} y=-B_{1} y \\
& B_{y}=-B_{0}+\frac{\partial B_{y}}{\partial x} x=-B_{0}+B_{1} x,
\end{aligned}
$$

where $B_{0}$ and $B_{1}$ are the dipole and quadrupole coefficients of the field. With these fields (assuming no longitudinal or horizontal magnetic fields are present), the transverse equations of motion for a reference particle along the accelerator circumference become

$$
\begin{aligned}
x^{\prime \prime}+K_{x}(s) x=0, \quad \text { with } \quad K_{x}(s) & =\frac{1}{\rho(s)^{2}}-\frac{B_{1}(s)}{B \rho} \\
y^{\prime \prime}+K_{y}(s) y=0, \quad \text { with } \quad K_{y}(s) & =\frac{B_{1}(s)}{B \rho},
\end{aligned}
$$

where $x^{\prime}=d x / d s$ (see Fig. 1.3). The focusing functions $K_{x, y}(s)$ stress the analogy with a simple harmonic oscillator, in which the "spring constant" $K$ is now a function of the position $s$. The general equation of motion is then expressed as

$$
u^{\prime \prime}+K_{u}(s) u=0,
$$

where $u$ stands for either $x$ or $y$ in Eqs. 1.8. The function $K_{u}$ is at least periodic ${ }^{\dagger}$ in the accelerator circumference, L. Equation 1.9 is known as the Hill's equation, extensively studied during the $19^{\text {th }}$ century. A solution of this equation reads

$$
u(s)=A_{u} \sqrt{\beta_{u}(s)} \sin \left(\psi_{u}(s)+\psi_{0}\right),
$$

where $A_{u}$ is just a constant depending on the initial conditions, and $\psi(s)$ is the phase advance. The function $\beta_{u}(s)$ modulates the oscillation's amplitude and it is called beta function, $\beta$ function, or the envelope function. It should not be confused with the relativistic speed $\beta=v / c$ in Eq. 1.4.

Inserting Eq. 1.10 into Hill's equation (Eq. 1.9) leads to the definition of another important parameter in accelerator physics, the betatron tune, $Q$ defined as the number of oscillations per revolution around the accelerator;

$$
Q_{u}=\frac{\psi_{u}(L)}{2 \pi}=\frac{1}{2 \pi} \int_{s_{0}}^{s_{0}+L} \frac{d s}{\beta_{u}(s)} .
$$

Equation 1.11 suggests that the $\beta$ function can be interpreted as the local wavelength of the oscillation. Depending on the literature, the tune of the accelerator is denoted by $\nu$ or $Q$. Other important parameters related to the $\beta$ function are:

$$
\begin{aligned}
& \alpha(s)=-\frac{\beta^{\prime}(s)}{2} \\
& \gamma(s)=\frac{1+\alpha(s)^{2}}{\beta(s)},
\end{aligned}
$$

\footnotetext{
${ }^{\dagger}$ Often, accelerators are designed with higher periodicity to ease design and operation
} 
which are collectively known as the Courant-Snyder, or Twiss parameters. Again, note that $\gamma(s)$ is not the relativistic $\gamma$ in Eq. 1.4. The importance of these parameters lies on the fact that at any location around the ring $s$, a trajectory in the phase space $\left(u, u^{\prime}\right)$ has an area bounded by a curve

$$
J=\gamma(s) u^{2}+2 \alpha(s) u u^{\prime}+\beta(s) u^{\prime 2},
$$

where $J$ is the single particle action, a constant of motion. It is also called the Courant-Snyder invariant. It is useful to characterize the beam by a particle that has an action that encloses a given fraction of the beam. For example, for a gaussian distributed beam this is the area in phase space containing the $34 \%$ of the beam particles. This is called the unnormalized rms emittance, $\epsilon_{\text {rms }}$. A widely used convention is

$$
\epsilon=6 \pi \epsilon_{\mathrm{rms}},
$$

known as the $95 \%$ emittance of the beam. That is, $95 \%$ of the beam particles in a gaussian beam have an action smaller than $\epsilon$. The rms transverse beam size is given by ${ }^{\ddagger}$

$$
\sigma_{t}(s)=\sqrt{\epsilon_{\mathrm{rms}} \beta(s)} .
$$

Note that the variables $\left(u, u^{\prime}\right)$ are not canonically conjugate, as opposed to $\left(u, p_{u}\right)$ (particle position and momentum). The relation between $p_{u}$ and $u^{\prime}$ is

$$
u^{\prime}=\frac{p_{u}}{p}=\frac{p_{u}}{\gamma_{r} \beta_{r} m c},
$$

where the subscript $r$ has been introduced in $\gamma_{r}$ and $\beta_{r}$ to denote their relativistic meaning. The factor $\beta \gamma$ increases when the beam is accelerated, causing a decrease in $u^{\prime}$ and hence reducing $\epsilon$. The normalized emittance,

$$
\epsilon_{n}=\gamma_{r} \beta_{r} \epsilon,
$$

is invariant under acceleration, and eases the comparison of emittances at different energies. In terms of the normalized emittance, the rms beam size is given by

$$
\sigma_{t}=\sqrt{\frac{\epsilon_{n} \beta}{6 \pi \beta_{r} \gamma_{r}}} .
$$

\subsubsection{Electric fields and longitudinal particle motion}

So far only the influence of the magnetic field in Eq. 1.2 has been considered. In the following, a brief review of the physics involved in the acceleration process and longitudinal capture/stability is presented by means of the electric fields. The active accelerating elements are the radio frequency $(\mathrm{rf})$ cavities, characterized by the frequency $\omega_{\mathrm{rf}}$ of the

\footnotetext{
${ }^{\ddagger}$ Dispersive terms and/or energy spread have been neglected in this analysis for convenience, and thus are not included in Eq. 1.15. For a more thorough analysis, see Refs. [14-16].
} 
longitudinal electric field oscillations and the peak voltage $V$. As for the transverse motion in Section 1.2.1, a more detailed description can be found in [14-16], on which the treatment in this section is based. Next and unless otherwise stated, the symbols $\beta$ and $\gamma$ used in the following refer to the relativistic velocity and Lorentz factor (Eq. 1.4), not to the Twiss parameters.

\section{Motion of the synchronous particle}

The fractional deviation of the particle's path length divided by the fractional deviation of the momentum is called the momentum compaction factor,

$$
\alpha_{p}=\frac{d L / L}{d p / p}=\frac{p d L}{L d p},
$$

where $L$ is the circumference of the design orbit. The angular frequency of a particle circulating in a synchrotron is:

$$
\omega=\frac{2 \pi}{\tau}=\frac{2 \pi \beta c}{L}
$$

where $\tau$ is the revolution period. Differentiation of Eq. 1.20 yields

$$
\frac{d \tau}{\tau}=\frac{d L}{L}-\frac{d \beta}{\beta}=\left(\alpha_{p}-\frac{1}{\gamma^{2}}\right) \frac{d p}{p} .
$$

The expression within parentheses is usually written as

$$
\eta \equiv \frac{1}{\gamma_{t}^{2}}-\frac{1}{\gamma^{2}}
$$

and it is called the slip factor. Notice that at a particular energy $\gamma=1 / \sqrt{\alpha_{p}}=\gamma_{t}$, the slip factor $\eta$ is zero. This is called the transition energy, whence the subscript $t$. Equation 1.22 yields a relation between the fraction revolution period deviation with the fractional momentum deviation given by

$$
\frac{d \tau}{\tau}=\eta \frac{d p}{p}
$$

Consider an rf cavity producing a wave of frequency $\omega_{\text {rf }}$, and assume that it is an integer multiple of a particle's angular revolution frequency $\omega_{0}$, such that

$$
\omega_{\mathrm{rf}}=h \omega_{0},
$$

for some integer $h$, which is called the harmonic number. The subscript 0 in Eq. 1.24 has been added to denote the synchronous particle, that is, a particle that crosses the rf cavity gap on every turn when the rf phase is $\psi_{s}$ and the voltage across the gap is $V \sin \psi_{s}$. The energy gained by the synchronous particle per revolution is

$$
\Delta E_{s}=q V \sin \psi_{s}
$$


However, not all the particles are synchronized with the rf field. So, it is useful to define the physical quantities of a particle relative to the synchronous one. Defining the relative phase difference $\phi=\psi-\psi_{s}$, and the difference in energy as $\Delta E=E-E_{s}$, the equations of motion for a particle with respect to the synchronous particle are:

$$
\begin{aligned}
\frac{d \phi}{d t} & =\frac{\eta \omega_{\mathrm{rf}} \tau}{\beta^{2} E_{s}} \Delta E \\
\frac{d \Delta E}{d t} & =e V\left(\sin \phi-\sin \phi_{s}\right) .
\end{aligned}
$$

For small phase oscillations, when $\sin \phi \approx \phi$, the two Eqs. 1.26 are merged in one,

$$
\frac{d^{2} \phi}{d t^{2}}+\left(2 \pi Q_{s}\right)^{2} \phi=0,
$$

where $Q_{s}$ is the synchrotron tune,

$$
Q_{s}=\sqrt{-\frac{\eta \omega_{\mathrm{rf}} \tau q V \cos \phi_{s}}{4 \pi^{2} \beta^{2} E_{s}}} .
$$

\section{Buckets and bunches}

The phase oscillations described in Eq. 1.26 are represented in Fig. 1.4. The unsynchronized generic particle B (which arrives at the rf station with a phase difference $\phi$, left) receives a kick larger than the synchronous particle. At the next rf station, the particle will arrive before the synchronous particle and it will receive a lower energy kick. This translates into a rotation around the synchronous particle on the longitudinal phase space plot (Figure 1.4, right). For a given set of rf parameters (voltage and harmonic number), unsynchronized particles within a certain area in phase space will undergo stable motion about the synchronous particle. The phase space trajectory of the particle that encloses the maximum area for stable motion is called the separatrix (Fig. 1.4, right), which defines the rf bucket. Because of this longitudinal stability, particles inside the bucket rotate around the synchronous particle and constitute a bunch.

These buckets exist as soon as the rf voltage is applied to the cavities. The number of buckets is given by $\omega_{\mathrm{rf}} / \omega_{s}=h$, the harmonic number (Eq. 1.24). In principle, one can fill as many of these buckets with bunches as one wishes. However, Table 1.3 shows that RHIC has an harmonic number of 360 with a bucket length of 35.6 ns. Since the injection kickers have a rise time of $90 \mathrm{~ns}[7]$, and bunches are transferred one-by-one, injection is only possible every third bucket. This means that the maximum number of bunches (filled buckets) allowed is 120. The second limitation is due to the abort kickers, which need a gap of $\approx 1 \mu$ s (about 30 buckets) in order to rise to full current. These two constraints limit the maximum number of bunches injected in RHIC to 110, with a minimum spacing between bunches of $107 \mathrm{~ns}$. 

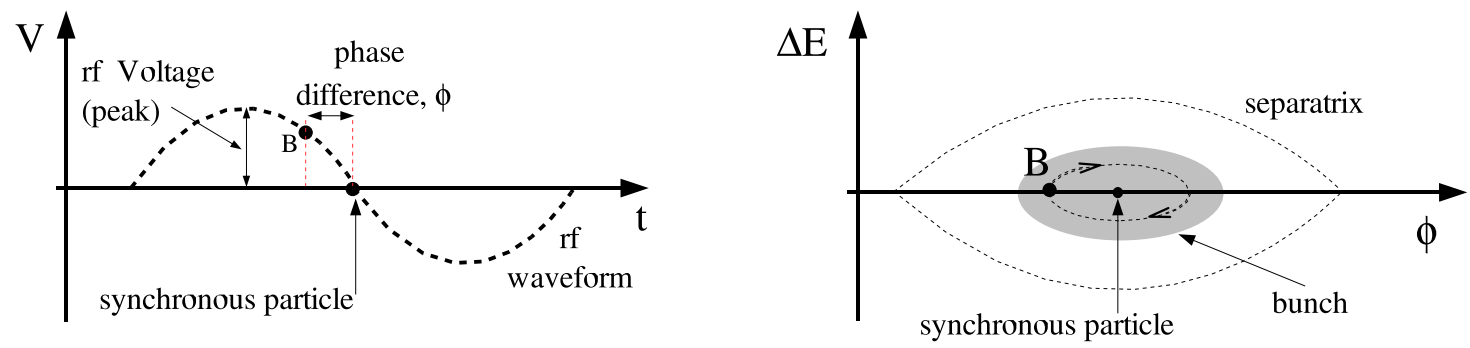

Figure 1.4: Bunch, bucket, separatrix. This is a non-accelerating bucket $\phi_{s}=0$.

\subsection{Consequences of a pressure rise}

One of the major concerns in this work is high pressure inside the RHIC vacuum chamber. When the pressure exceeds the $10^{-5}$, the ion pumps do not work any more and all the pumping speed can be lost. Below this limit, other detrimental consequences exist.

\subsubsection{Beam lifetime}

A beam with initial intensity $I_{0}$ circulating for a time $t$ inside an accelerator decays exponentially like

$$
I=I_{0} e^{-n \sigma_{\text {tot }} \beta c t}
$$

where $I_{0}$ is the initial beam intensity, $\sigma_{\text {tot }}$ is the total cross section for all beam-gas interactions, and $n$ is the gas molecule density. The gas density is related to the pressure through $n=P / k T$, where $\mathrm{k}$ is the Boltzman's constant and $T$ is the system temperature. The beam intensity lifetime is given by

$$
\tau=\frac{k T}{\beta c \sigma_{\mathrm{tot}} P}
$$

When there are many processes of comparable significance, the cross sections are added:

$$
\sigma_{\mathrm{tot}}=\sigma_{1}+\sigma_{2}+\cdots
$$

and the total lifetime is then obtained adding the inverse of the individual lifetimes,

$$
1 / \tau=1 / \tau_{1}+1 / \tau_{2}+\cdots
$$

The possible processes affecting the beam lifetime in an accelerator include inelastic interactions (bremsstrahlung, electron capture/loss, nuclear reactions) or elastic ones (coulomb scattering) [17]. Based on the relative cross sections, the ones affecting RHIC the most are the elastic single Coulomb scattering processes [18]. The cross sections depend on the beam ion species and rest gas composition. They are calculated in a "billiard ball" model:

$$
\sigma_{N}=\pi r^{2}, \text { with } \quad r=r_{0}\left(A_{b}^{1 / 3}+A_{t}^{1 / 3}\right),
$$


where $r_{0}=1.2 \times 10^{-15} \mathrm{~m}$ is the Fermi radius, and $A_{b}$ and $A_{t}$ are the atomic masses for the beam and the target rest gas molecule, respectively.

Table 1.2 lists the cross section values and beam lifetime assuming a different gas composition for the cold and warm regions. For the warm sections, the gas composition is assumed to be a $90 \% \mathrm{H}_{2}, 5 \% \mathrm{CO}$, and $5 \% \mathrm{CH}_{4}$. For the cold regions, it is assumed to be $100 \%$ of Helium. Since the lifetime scales as $\langle P\rangle^{-1}$, it is easy to see that for an average warm pressure $\langle P\rangle=5 \times 10^{-7}$ Torr, the beam gold lifetime decreases to $\approx 0.6$ hours.

Table 1.2: Relevant parameters for the lifetime calculations.

\begin{tabular}{|l|c|c|c|c|c|}
\hline \hline & $\mathrm{T}(\mathrm{K})$ & length $(\mathrm{m})$ & $\langle P\rangle$ (Torr) & $\sigma_{N}$ (barns) & $\tau$ (hours) \\
\hline warm & 300 & 760 & $5 \times 10^{-10}$ & 4.7 & 600 \\
cold & 4 & 3100 & $10^{-11}$ & 2.7 & 242 \\
\hline \hline
\end{tabular}

\subsubsection{Experimental backgrounds}

Unwanted background noise in the experimental detectors due to beam-residual gas interactions puts the most stringent requirements on RHIC vacuum [19]. Assuming an interaction region of length $l$, the number of interactions per unit of time, or rate $R$, produced by a beam with intensity $I$ is:

$$
R=\frac{P}{k T} \sigma_{N} l I / e,
$$

where $e$ is the absolute value of an electron charge. The beam-beam rates are $R_{b}=\sigma_{b, b} L$, where $L$ is the luminosity and $\sigma_{b, b}$ is the beam-beam cross section. Calculating $\sigma_{b, b}$ with Eq. 1.33, for a typical luminosity value of $10^{27} \mathrm{~cm}^{-2} \mathrm{~s}^{-1}$, and a typical pressure of $5 \times 10^{-10}$ Torr, and assuming an interaction length $l=20 \mathrm{~m}$, the ratio $R / R_{b, b} \approx 1$ (both in the $\mathrm{kHz}$ range). Thus, although the calculated beam lifetime is about $600 \mathrm{~h}$ for an average pressure of $5 \times 10^{-10}$ Torr in the warm regions, it is desired to reduce that value by more than one order of magnitude in the interaction regions. These requirements can be relaxed if the experimental detectors are appropriately shielded, or if larger beam-gas background rates are tolerable.

\subsubsection{Emittance growth}

Finally, elastic Coulomb scattering causes the transverse emittance of the beam to grow. The growth rate for gold beams at $\gamma=100$ is [18]

$$
\frac{d \epsilon_{n}}{d t} \approx 10^{-4} \mathrm{~mm} \mathrm{mrad} / \text { hour }
$$

for both warm and cold sections (using the average pressures in Table 1.2). This value is insignificant compared with the growth due to intrabeam scattering, which is measured in to be about $0.1 \mathrm{~mm} \mathrm{mrad} /$ hour [20]. 
Table 1.3: Major RHIC design parameters for gold ions, and protons. After a tune scan, betatron tunes of $(28.72,28.73)$ were used after Run 4 for protons only.

\begin{tabular}{|c|c|c|c|c|c|}
\hline & & & $\overline{\text { Gold }}$ & Protons & Copper \\
\hline parameter & symbol & unit & value & value & value \\
\hline mass number & $A$ & - & 197 & 1 & 63 \\
\hline atomic number & $Z$ & - & 79 & 1 & 29 \\
\hline number of ions/bunch & $N_{b}$ & $10^{9}$ & 1 & 100 & 4.5 \\
\hline $\begin{array}{l}\text { number of bunches/ring } \\
\text { circumference }\end{array}$ & $\bar{C}$ & $\begin{array}{c}- \\
\mathrm{m}\end{array}$ & \multicolumn{3}{|c|}{$\begin{array}{l}\text { variable, from } 28 \text { to } 110 \\
3833.85\end{array}$} \\
\hline $\begin{array}{l}\text { energy per beam injection } \\
\text { store }\end{array}$ & $E$ & $\mathrm{GeV} / \mathrm{u}$ & $\begin{array}{l}10.8 \\
100\end{array}$ & $\begin{array}{c}28.3 \\
100 \& 190\end{array}$ & $\begin{array}{l}12.6 \\
100\end{array}$ \\
\hline transition energy & $\gamma_{t}$ & $\mathrm{GeV} / \mathrm{u}$ & \multicolumn{3}{|c|}{22.89} \\
\hline $\begin{array}{l}\text { magnetic rigidity injection } \\
\text { store }\end{array}$ & $B \rho$ & $\mathrm{T} \mathrm{m}$ & $\begin{array}{c}81.1 \\
839.5\end{array}$ & $\begin{array}{c}81.1 \\
339.5\end{array}$ & $\begin{array}{c}81.1 \\
724.6\end{array}$ \\
\hline betatron tune $\begin{array}{l}\text { horizontal } \\
\text { vertical }\end{array}$ & $\begin{array}{l}Q_{x} \\
Q_{y}\end{array}$ & - & \multicolumn{3}{|c|}{$\begin{array}{l}28.19 \\
28.18 \\
\end{array}$} \\
\hline$\beta$ function at crossing $\begin{array}{l}\text { injection } \\
\text { store }\end{array}$ & $\beta^{*}$ & $\mathrm{~m}$ & \multicolumn{3}{|c|}{$\begin{array}{c}10 \\
1\end{array}$} \\
\hline harmonic number $\begin{array}{l}\text { injection } \\
\text { store }\end{array}$ & $h$ & - & $\begin{array}{c}360 \\
2520\end{array}$ & $\begin{array}{l}360 \\
360\end{array}$ & $\begin{array}{c}360 \\
2520\end{array}$ \\
\hline $\begin{array}{l}\text { rf voltage } \\
\text { storection }\end{array}$ & $V$ & MV & $\begin{array}{c}0.3 \\
6\end{array}$ & $\begin{array}{c}0.3 \\
3\end{array}$ & $\begin{array}{c}0.3 \\
6\end{array}$ \\
\hline $\begin{array}{ll}\text { rf frequency injection } \\
\text { store }\end{array}$ & $\omega_{\mathrm{rf}}$ & $\mathrm{MHz}$ & $\begin{array}{c}28.15 \\
198\end{array}$ & $\begin{array}{l}28.15 \\
28.15\end{array}$ & $\begin{array}{c}28.15 \\
198\end{array}$ \\
\hline $\begin{array}{l}\text { synchrotron freq. } \\
\text { injection } \\
\text { store }\end{array}$ & $Q_{s}$ & $\mathrm{~Hz}$ & $\begin{array}{l}120 \\
333\end{array}$ & $\begin{array}{c}55 \\
388\end{array}$ & $\begin{array}{l}145 \\
270\end{array}$ \\
\hline $\begin{array}{c}\text { injection } \\
\text { bunch area } \\
\text { store }\end{array}$ & $S_{95 \%}$ & $\mathrm{eV} \mathrm{s} / \mathrm{u}$ & $\begin{array}{l}0.5 \\
1.1\end{array}$ & $\begin{array}{l}0.5 \\
1.2\end{array}$ & $\begin{array}{l}0.7 \\
1.0\end{array}$ \\
\hline normalized emittance & $\epsilon_{n}$ & $\mathrm{~mm}$ mrad & $10 \pi$ & $20 \pi$ & $10 \pi$ \\
\hline energy spread & $\Delta E / E$ & - & \multicolumn{3}{|c|}{$<10^{-3}$} \\
\hline
\end{tabular}





\section{Chapter 2}

\section{Electron cloud effect in accelerators}

Electric fields present a vacuum chamber may accelerate electrons (produced by field emission, photo-emission, residual gas ionization, etc) towards the wall chamber surface. If the bombarding electrons acquire enough energy, they produce secondary electrons when they hit the chamber wall, which in turn are accelerated by the electric field normal to the surface. These electrons may bombard again another surface and emit secondary electrons. If the appropriate combination of surface properties and electric fields are fulfilled, this bouncing back and forth between surfaces develops an electron multiplication, or multipacting effect, which creates a cloud of electrons inside the vacuum chamber. The electron cloud in accelerators is defined as an accumulation of electrons inside the beam pipe which, if sufficiently strong, can affect the machine performance by increasing the vacuum pressure, producing emittance growth, causing beam loss, cryogenic heat load or interference of the beam diagnostics [2].

\section{$2.1 \quad$ Introduction}

In the field free regions of accelerators, the electric field producing the multipacting is provided by the beam*, so that the two components influencing the electron accumulation are the beam and the chamber surface characteristics. Among this, the most relevant is the Secondary Electron Yield (SEY, or $\delta$ ) of the chamber material and its dependence on the impinging electron energy. An example of this dependence is shown in Fig. 2.1. For a given electron, more than one secondary electron are produced if the electron energy is larger than $E_{1}$ and smaller than $E_{2}$. Motivated by the predicted detrimental consequences on the future LHC collider at CERN, the electron cloud has been studied using RF test benches in laboratory set-ups $[21,22]$. However, the conditions in an accelerator are different. Next, the electron cloud characterization depending on the accelerator beam parameters is shown. The electron cloud is mainly classified into two different regimes: single-bunch and multi-bunch regime.

*For the regions with electric or magnetic elements (such as RF cavities, bending magnets, et cetera), the field becomes a combination of beam and the external field for each case. 


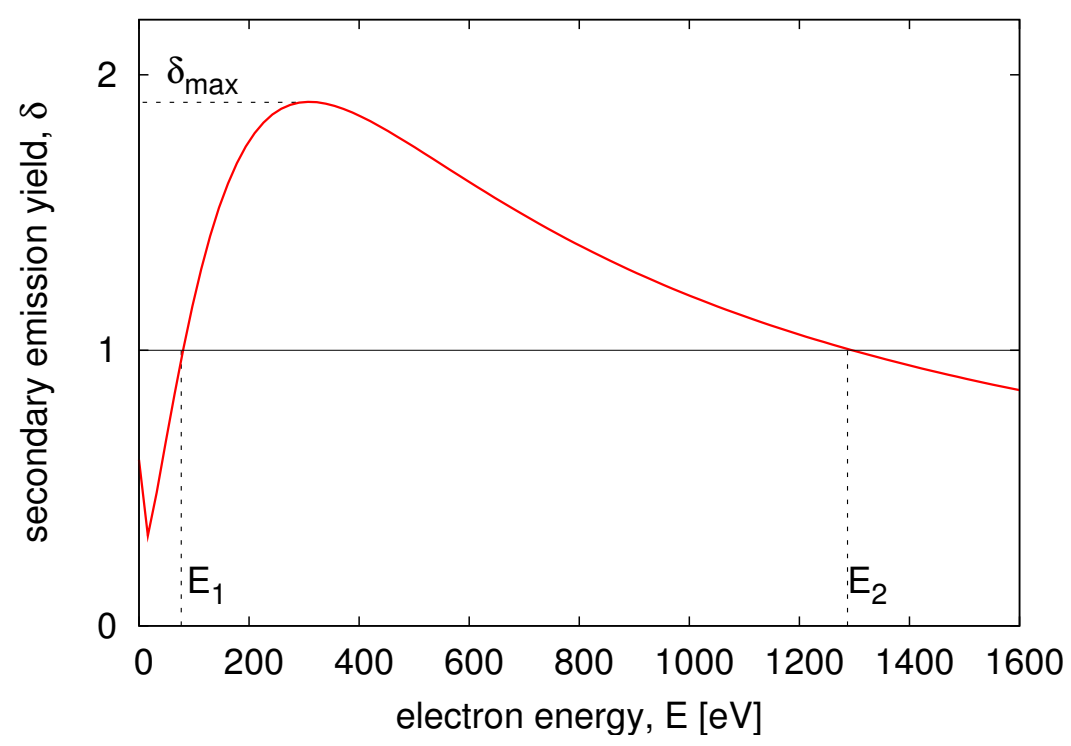

Figure 2.1: Typical behaviour of the secondary electron yield as a function of the impinging electron energy. The behaviour changes for different materials, with different $\delta_{\max }, E_{1}, E_{2}$, etc.

\subsection{Single-bunch regime}

The single-bunch regime occurs if the length of the (positively charged) bunch is long enough to sustain multiple passes of electrons. It is also called trailing edge multipacting. Electrons are attracted by the beam potential, and their motion is characterized by the electron bouncefrequency [23],

$$
\omega_{e}=c \sqrt{2 \pi r_{e} \lambda_{p}}
$$

where $r_{e}$ is the classical electron radius, and $\lambda_{p}$ is the volume density of the beam. After the passage of the bunch density peak, electrons are released and accelerated by the part of the beam of decreasing density (trailing edge of the beam density distribution, see Fig. 2.2). The number of electrons inside the chamber grows dramatically upon such trailing edge multipacting, as observed at the PSR and expected in the SNS ring [24]. The build-up is typically not sensitive to the bunch spacing, and it critically depends on the length of the bunch and the variations in its longitudinal density, which determine the energy gain and the multipacting duration. Upon acceleration by the beam, the electron energy is typically below the keV level. Associated with the electron cloud build up, pressure rises, and single-bunch, transverse instabilities are observed [25]. 


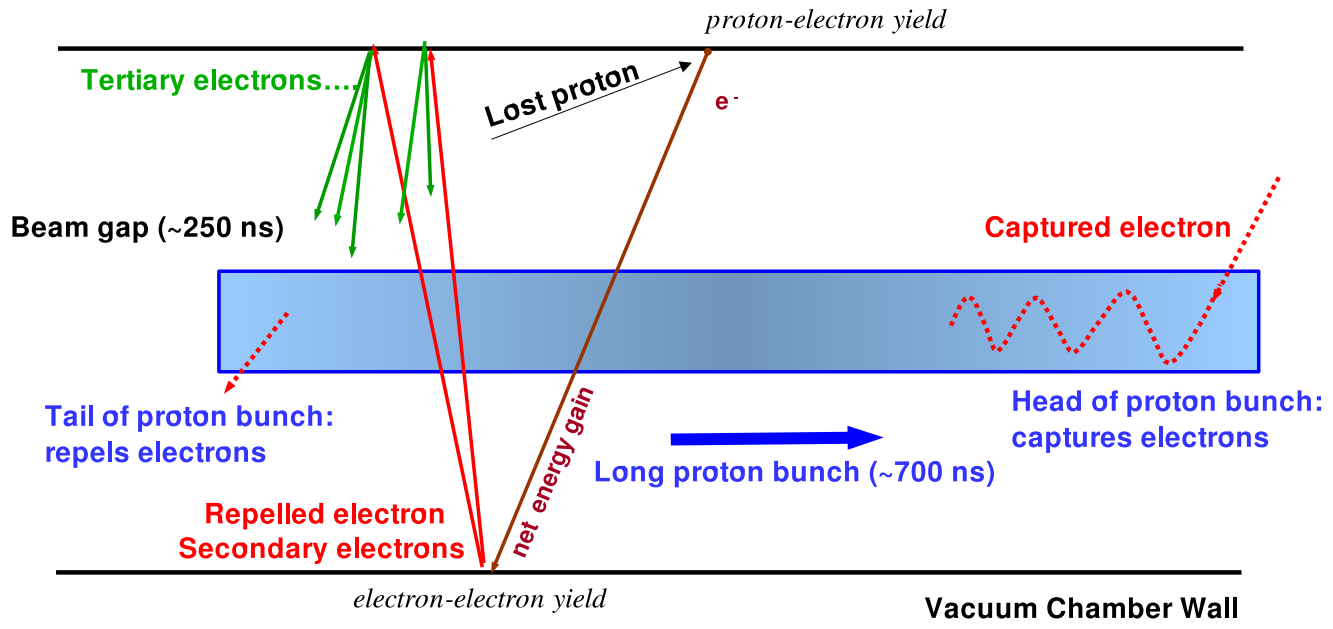

Figure 2.2: Single-bunch multipacting schema at the PSR. Courtesy of J. Wei [25].

\subsection{Multi-bunch regime}

In this case, the electron accumulation occurs under the passage of successive positively charged bunches $[2,4,26]$. The primary electrons can be created by photoelectrons (produced from synchrotron radiation), ionization of residual gas, and when stray particles hit the chamber walls. These primary electrons gain energy due to the interaction with the passing bunch, or under successive bunch passages. A multipacting effect can develop if two conditions are fulfilled:

- the energy gain is such that it can generate more than one secondary electron after impinging the chamber wall. In Fig. 2.1, this is at an energy larger than $E_{1}$, and smaller than $E_{2}$.

- the survival time of these secondary electrons is comparable to the bunch spacing.

This mechanism was first described as beam-induced multipacting (BIM) in Ref. [4]. For a round chamber of radius $b$, BIM was historically presented as almost a resonance: the time by which the electrons cross the vacuum pipe is in synchronism with the bunch passages, which leads to $[4,26]$

$$
N_{b} \leq \frac{b^{2}}{r_{e} s_{b}},
$$

where $N_{b}$ is the bunch population, and $s_{b}$ is the bunch spacing (in units of length). However, Eq. 2.2 is far too stringent. Since most of the secondary electrons travel with very low energy, and in addition, the SEY for this low energy is close to unity, their survival time can be very long. Thus electron clouds occur even for bunch spacings much larger than that suggested by Eq. 2.2. Electron clouds are observed in machines with short bunch spacings, $\left(s_{b} / c \approx 4-8 \mathrm{~ns}\right.$, like with the positron beams in KEK-B and PEP-II), and long bunch spacings 


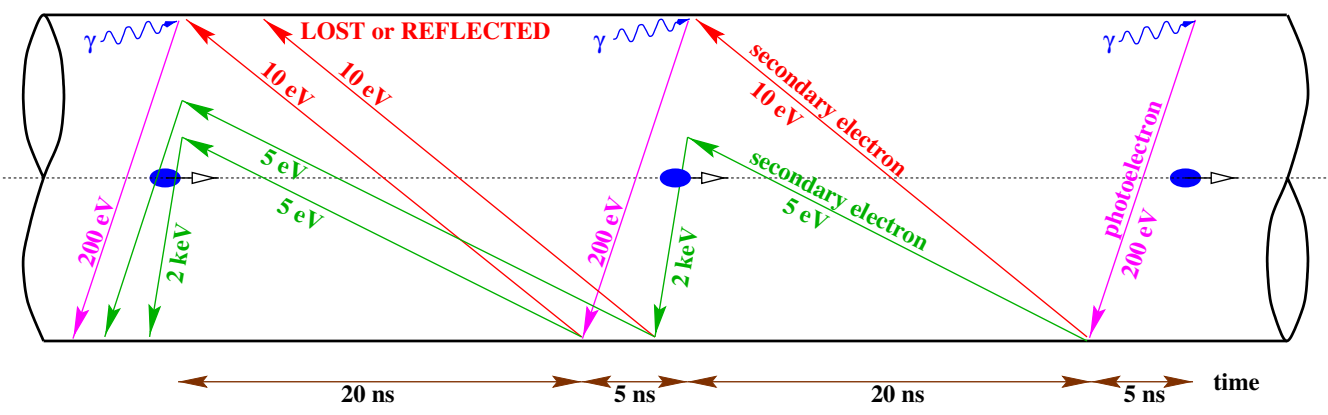

Figure 2.3: Multipacting schema in the LHC. Courtesy of F. Ruggiero.

$\left(s_{b} / c \approx 25\right.$-200 ns, like with the hadron beams in SPS or RHIC). An interesting result is that, due to the purely electromagnetic nature of the interaction driving the electron cloud mechanism, electron clouds have also been found with electron beams at the APS [27], albeit at a more modest level compared to the positron beams in the same machine.

\subsection{Electron Cloud at RHIC}

The electron cloud in RHIC falls primarily in the multi-bunch regime, although it is also categorized in an intermediate regime for sharing some attributes of both categories $[8,28,29]$. The process is depicted in Fig. 2.4. Primary electrons are produced either by beam ionization of the residual gas, or by beam lost particles striking the chamber wall at grazing incident angles [30]. If an electron is close to the wall, in most cases it only changes its kinetic energy without noticeably changing its position during a bunch passage. On the other hand, a primary electron near the beam will be trapped inside the bunch potential, performing similar oscillations as in the single-bunch regime. Its final energy gain depends on its initial transverse position, bunch population, and bunch profile. This situation was called "kick" (for electrons that do not change its position) and "autonomous" (for electrons trapped inside the bunch) in Ref. [31], as it will be discussed in Chapter 3.

If the two conditions expressed in Section 2.3 are fulfilled, an electron cloud develops. Figure 2.4 shows that the source of primary electrons in RHIC comes from the electron yield by beam ions striking the surface wall, and beam-gas ionization. The multiplication depends on beam parameters (like bunch spacing, bunch population, bunch length, etc), and chamber characteristics (beam pipe radius, SEY, reflection probability at low energy, et cetera). It should be stressed that for RHIC, and many other machines with electron clouds, the secondary electron emission process has a more significant effect on the overall electron density than the primary source mechanism $[8,32]$. 


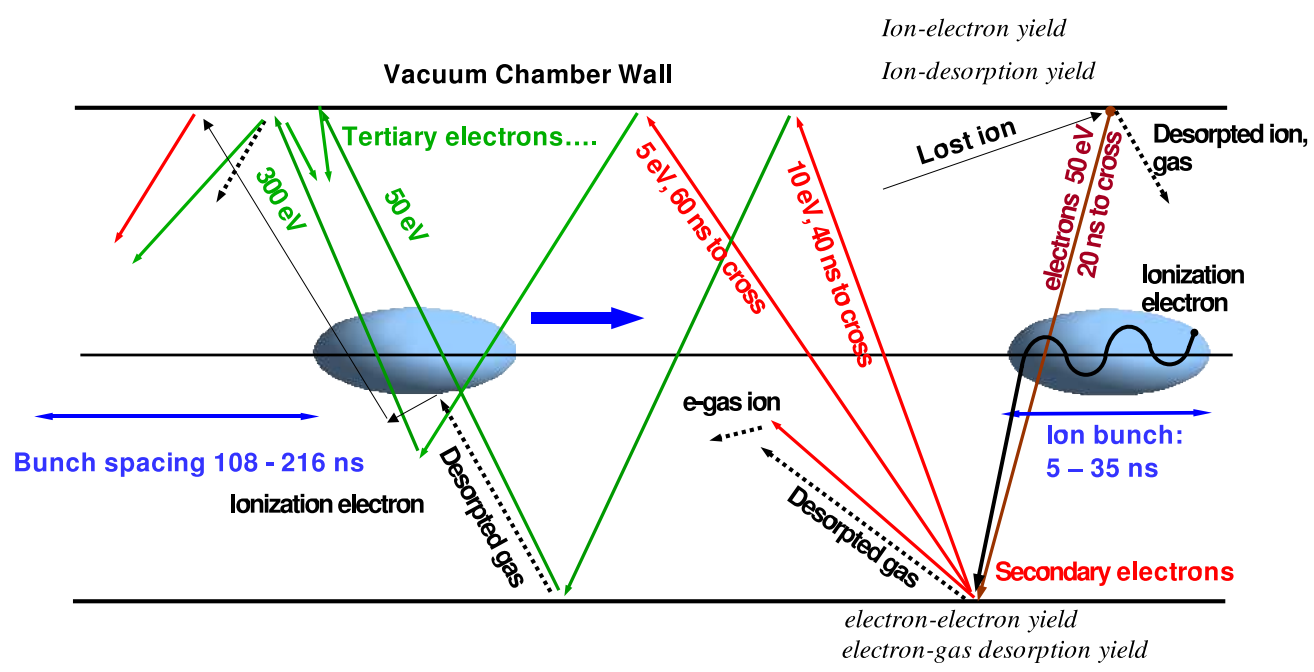

Figure 2.4: RHIC multipacting schema. Primary electrons are typically produced either by beam ions striking the chamber wall, or by beam ionization of the residual gas, and are accelerated by the bunch potential.

\subsection{Pressure rises in RHIC during Run-2}

During Run-2 in 2001, the number of ions per bunch was continually increased up to the design value of $10^{9}$ at the end of the run. Operation with both 56 and 110 bunches/beam led to intolerable vacuum pressure rises in the warm bore [33-35]. Figure 2.5 shows an sketch of RHIC with a zoom for half of its sextants (bottom plot). In both the yellow and blue rings, intolerable pressure rises are concentrated in the two warm bores: the $34 \mathrm{~m}$ between the triplets and the "Q4" magnet, and the $\approx 17 \mathrm{~m}$ in the experimental region. Up to 2002, no pressure rises or heat loads were detected in the cold bores.

Figure 2.6 shows a typical evolution of the pressure rise during a high beam intensity injection. In the first attempt, a total of 39 bunches spaced 107 ns apart with $0.75 \times 10^{9}$ $\mathrm{Au}$ ions/bunch are injected into the blue ring, producing a violent pressure rise up to $7 \times 10^{-7}$ Torr. When the bunch spacing is doubled (from 107 to $214 \mathrm{~ns}$ ), the injection is completed and the pressure rise in the single (blue) beam pipe reaches "only" the $10^{-8}$ Torr level. When the yellow beam is injected, the pressure in the common beam pipes grows by about 4 orders of magnitude up to the vacuum trip limit of $10^{-5}$ Torr, at which the beam is dumped. The pressure sampling during Run- 2 is relatively slow ( 5 seconds per sample). The pressure rises in Fig. 2.6 are sensitive to the bunch intensity and spacing [34], a common electron multipacting signature [2,32] (as will be seen in Chapters 3 and 10). A second mechanism related to molecular desorption induced by beam loss is also suggested in Ref. [34]. This possibility is further addressed in Chapter 7.

Since no dedicated electron detectors were available in 2001, the role of electron clouds in the pressure rise during Run-2 was first investigated through beam measurements. Figure 2.7 shows the coherent tune measured along a train of 110 bunches spaced by $107 \mathrm{~ns}$ in the 


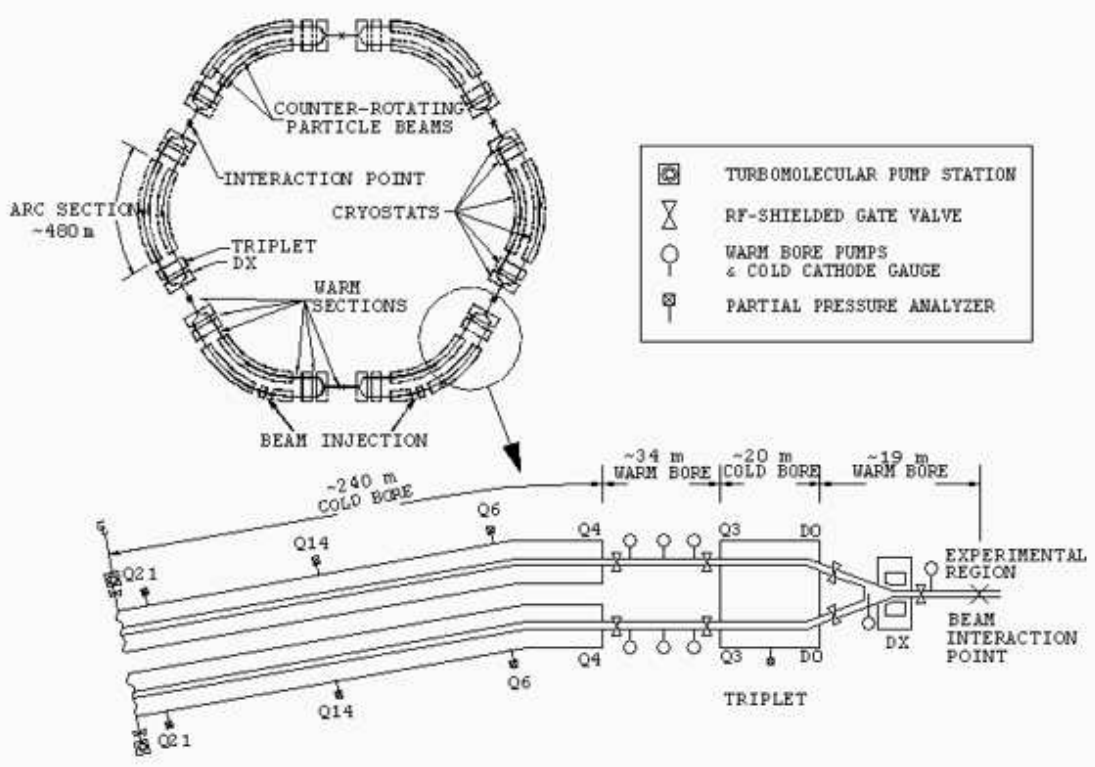

Figure 2.5: The top plot shows a sketch of the RHIC collider [7]. The bottom plot corresponds to a zoom for half of the machine sextants marking the location of the vacuum components. The machine has a six-fold symmetry and the zoomed structure is repeated along the circumference.

yellow ring. In this case, the injection of 110 bunches with an average intensity of $3 \times 10^{10}$ protons/bunch produces a horizontal and vertical tune shift of about $2 \times 10^{-3}$. The electron cloud density $\rho_{e}$ (in units of $\left[\mathrm{m}^{-3}\right]$ ) is estimated from the coherent tune shift along the bunch train by $[36]$

$$
\Delta Q_{\mathrm{x}, \mathrm{y}}=\rho_{e}\left(\frac{r_{p} Z}{\gamma A}\right) \frac{h_{\mathrm{x}, \mathrm{y}} \beta_{\mathrm{x}, \mathrm{y}} L^{\prime}}{h_{x}+h_{y}}
$$

where $h_{\mathrm{x}, \mathrm{y}}$ is the chamber aperture, $\beta_{\mathrm{x}, \mathrm{y}}$ is the average $\beta$ function in the horizontal/vertical direction, and $L^{\prime}$ refers to the length of the ring in which electron cloud is present.

The estimated electron cloud densities using Eq. 2.3 are compared with simulation results for RHIC in Ref. [8], whose calculations predict electron cloud densities in the range 0.2 to $2 \mathrm{nC} / \mathrm{m}$. Electron cloud densities of the same order of magnitude are reproduced using simulations, although the beam intensities are slightly larger in the simulations than in the measurements, indicating either that some physics is missing in the model, or that there is an insufficient knowledge of the wall surface parameters. Simulation results are very sensitive to the variation of many parameters [8]. This method had been previously applied in other machines, like KEK $[37,38]$ and the SPS $[39,40]$.

The sensitivity of the pressure rise to bunch intensity and spacing [34,35], and the tune shift analysis and simulations [8] point to the electron cloud effect as the driving mechanism for the observed pressure rises. The influence of other mechanisms, either directly related to 


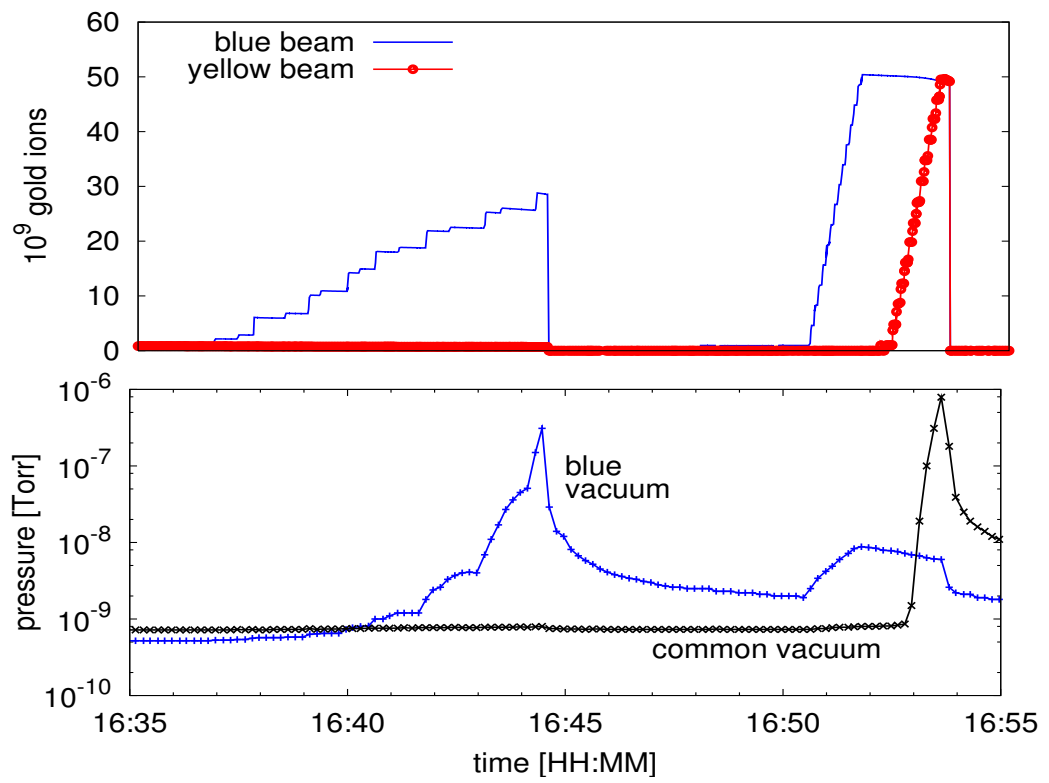

Figure 2.6: Two attempts to inject high intensity beams during fill 1797 (in 2001). In the first attempt (at 16:40), only 39 bunches with a bunch spacing of $107 \mathrm{~ns}$ can be injected in the blue ring because of the large pressure rise (bottom plot). By doubling the bunch spacing to $214 \mathrm{~ns}$, the pressure in the blue vacuum pipes is two orders of magnitude lower, and both beams can be filled.

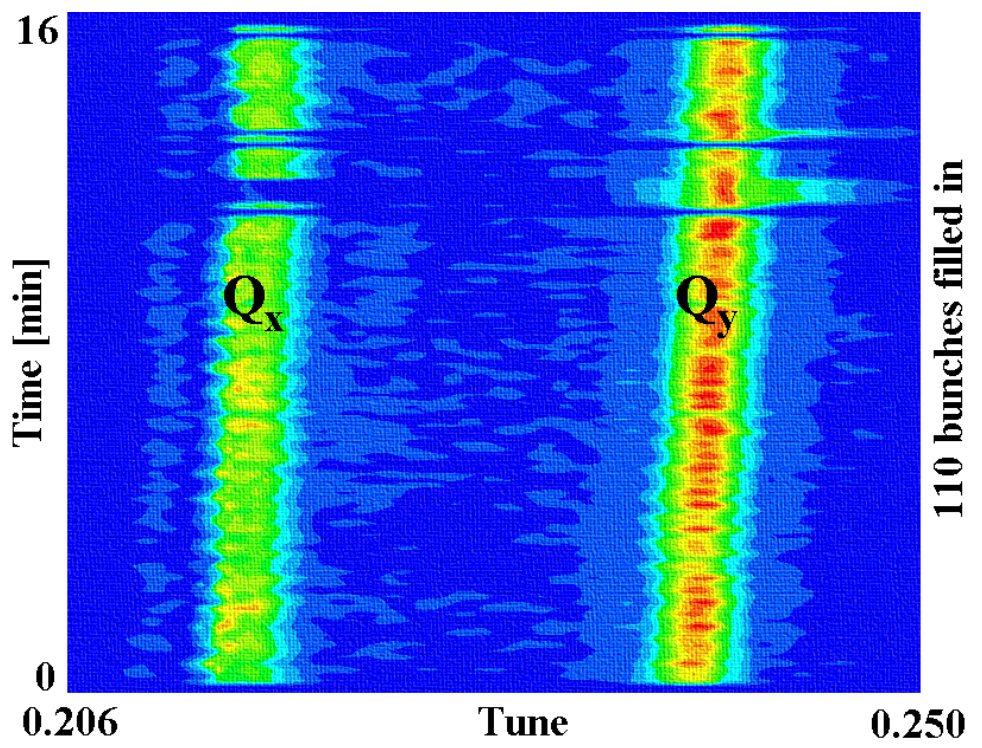

Figure 2.7: Coherent tunes measured along the bunch train of 110 bunches with 107 ns bunch spacing. Both the horizontal $\left(Q_{x}\right)$ and vertical $\left(Q_{y}\right)$ tunes increase as the bunches are being injected due to the increase of electron density. Figure courtesy of W. Fischer [8]. 
the electron cloud itself or to the pressure rise, had to be properly analyzed with dedicated instrumentation.

In particular, a crucial instrument installed for Run-3 is the electron detector presented in Chapter 4. With this detector, a value for the electron induced molecular desorption coefficient is inferred in Chapter 5, and the electron cloud computer simulation codes are benchmarked with experimental data in Chapter 6. Chapter 7 reports the difference between single and common beam pipes, and Chapter 8 shows some cures against the electron multipacting in accelerators.

A new way to treat the electron cloud through the use of maps is introduced in Chapter 9, rendering results otherwise difficult to obtain. For a given surface and beam characteristics, Chapter 10 shows how to calculate the effective secondary emission yield of the surface and its relation with the maps for electron clouds. Finally, Chapter 11 shows the possibility of treating coupled electron and ion clouds using simple maps and the interesting results it yields. 


\section{Chapter 3}

\section{Electron cloud build-up mechanism}

This chapter contains an overview of the physics involved in the build up of an electron cloud. The first problem to be addressed is the interaction between an electron and a passing bunch in absence of external electromagnetic fields. Electron multiplication in the wall surface is then introduced by means of the Secondary Electron Yield (SEY or $\delta$ ) of the beam pipe wall, and the electron cloud build up is finally stopped due to the space charge effects of the cloud itself. However, so far there is not an analytical process for merging these processes, and the electron cloud build up is currently studied by means of several computer simulation codes. A description of one of these codes, CSEC, is given at the end of the Chapter.

\subsection{Primary electron sources}

Primary electrons are mainly generated by three processes: photoelectrons, ionization of the residual gas, and electrons generated when lost beam particles hit the chamber walls. For RHIC (as for all hadron machines but the LHC) the photoelectrons are not a significant source of primary electrons because the synchrotron radiation is small. The dominant sources of primary electrons in RHIC are the ionization of residual gas and electron production off the wall from stray beam particles. The rate of primary electrons per bunch passage per unit length created by beam-gas interactions is

$$
\frac{d N_{e}}{d l}=\sigma N_{b} \frac{P}{k T}
$$

where $\sigma$ is the residual gas ionization by the beam particles, $N_{b}$ is bunch population, $P$ is the vacuum pressure, $k$ is the Boltzmann constant and $T$ is the system temperature.

For beam protons or ions impacting on the wall, the electron generation rate (number of electrons per incident particle) is [30]

$$
\frac{d N_{e}}{d N_{b}} \approx \frac{\Lambda}{\cos \theta}\left(\frac{d E}{d x}\right),
$$

where $d E / d x$ is the electronic stopping power, $\theta$ is the incident angle, and $\Lambda$ is a constant depending on the material and the beam particle. This yield ranges between $\approx 200$ for protons 
Chapter 3. Electron cloud build-up mechanism

and $\approx 20000$ for gold particles at grazing incident angles $\theta \approx 89.6^{\circ}$. For angles larger than $\theta=89.8^{\circ}$, Eq. 3.2 is not valid because saturation is observed.

\subsection{The electron-bunch interaction}

Consider a circular cross section beam pipe with a highly relativistic positive ion bunch $(\beta=v / c \approx 1)$ moving in the longitudinal direction, $z$ (see Fig. 3.1). The Hamiltonian (expressed in cylindrical coordinates) describing the motion of an electron with mass $m_{e}$ and charge $-e$ is given by:

$$
H=\frac{p_{r}^{2}}{2 m_{e}}+\frac{p_{\theta}^{2}}{2 m_{e} r^{2}}+\frac{\left[p_{z}-e \beta_{e} V(r, z-\beta c t) / c\right]^{2}}{2 m_{e}}-e V(r, z-\beta c t),
$$

where $\beta_{e}=v_{e} / c$ is the relativistic speed of the electron, and $V(r, z-\beta c t)$ is the potential created by the bunch charge distribution. In order to simplify Eq. 3.3, it is convenient to study the contribution of the different parts in the Hamiltonian.

First, since the multipacting electrons are not relativistic $\left(v_{e} \ll \mathrm{c}\right)$, the term $e \beta_{e} V(r, z-\beta c t) / c$ can be neglected, as well as any bunch related magnetic effects on the electron dynamics. Second, it follows from classical electrodynamics [41], that the electric field for a highly relativistic proton bunch is Lorentz contracted to a cone with an rms angle of order $1 / \gamma$ in the direction perpendicular to the beam's motion, where $\gamma$ is the relativistic bunch's Lorentz. Thus, as a first approximation, the influence of the longitudinal kick produced by the beam can be neglected because its effect is small.

If the angular momentum $p_{\theta}$ is taken to be zero, the Hamiltonian simply becomes

$$
H=\frac{p_{r}^{2}}{2 m_{e}}-e V(r, z)
$$

The radial time evolution is followed using the well-known Hamilton equation [42]:

$$
\frac{\partial p_{r}}{\partial t}=-\frac{\partial H}{\partial r}=e \frac{\partial V(r, z)}{\partial t}
$$

To evaluate the potential $V(r, z)$, take a beam pipe wall with a perfectly conducting surface. The potential follows the Poisson equation

$$
\nabla^{2} V=-\rho / \epsilon_{0}
$$

From Gauss' theorem, assuming both a cylindrically symmetric beam-pipe and bunch distribution, this potential is given by

$$
V(r, z)=\frac{1}{\epsilon_{0}} \int_{0}^{r} r^{\prime} \rho\left(r^{\prime}, z\right) \ln \frac{r^{\prime}}{r} d r^{\prime}
$$

where $\rho(r, z)$ is the beam's spatial charge distribution. This charge distribution is factorized as:

$$
\rho(r, z)=\lambda_{b}(z) f(r)
$$




\subsubsection{Cylindrical bunch}

First, consider the naive but informative case in which the bunch takes the form of an infinitely long and uniformly charged cylinder of radius $\sigma_{r}$ and constant linear density $\lambda_{b}$. This approximation is valid for RHIC bunches because, considering the bunch shape is modeled by gaussian curves in the longitudinal and transverse plane, the rms bunch length $\sigma_{z}$ is significantly larger than the transverse size, $\sigma_{r}$. The linear charge density is then

$$
\lambda_{b}=\frac{Z e N_{b}}{\sqrt{2 \pi} \sigma_{z}},
$$

where $N_{b}$ is the number of charged particles in the bunch, and $Z$ is the charge ion state. In this case, the potential in Eq. 3.7 takes the following form,

$$
V(r)= \begin{cases}-\frac{\lambda_{b}}{4 \pi \epsilon_{0} \sigma_{r}^{2}} r^{2} & ; \quad \text { for } r \leq \sigma_{r} \\ -\frac{\lambda_{b}}{2 \pi \epsilon_{0}}\left(\ln \frac{r}{\sigma_{r}}+\frac{1}{2}\right) & ; \quad \text { for } r \geq \sigma_{r} .\end{cases}
$$

The dependence on $z$ is not present in this approximation. It is possible to distinguish two regimes in the electron-bunch interaction as a function of the initial electron position, $r$. One corresponds to the electrons outside the beam core that are nearly stationary during a bunch passage. The other corresponds to electrons that get trapped within the beam core. The latter regime has a similar potential to that of the harmonic oscillator.

S. Berg [31] defined these regimes as "kick", (electrons in the beam pipe do not get inside the bunch during a bunch passage), and "autonomous" (electrons in the beam pipe perform harmonic oscillations). The critical radius $r_{C}$ (see Fig. 3.1) separating these two regimes is defined as the radial distance for which the time for the bunch to pass is equal to a quarter* of the oscillation period. It can be calculated as [43]

$$
r_{C} \approx 2 \sqrt{Z N_{b} r_{e} \sigma_{z} \sqrt{2 / \pi}}
$$

where $r_{e}=e^{2} /\left(4 \pi \epsilon_{0} m_{e} c^{2}\right)=2.82 \times 10^{-15} \mathrm{~m}$ is the classical radius of the electron.

From the Hamilton Eqs. 3.5, the radial momentum gained by an electron is:

$$
\Delta p_{r} \approx e \frac{\partial V(r, z)}{\partial r} \Delta t_{b}
$$

where $\Delta t_{b}$ is the time it takes for the bunch to pass.

"Kicked" electrons initially close to the beam pipe wall gain an energy:

$$
\Delta E_{\mathrm{wall}}=2 m_{e} c^{2}\left(\frac{Z N_{b} r_{e}}{b}\right)^{2}
$$

${ }^{*}$ In a harmonic oscillator, the maximum energy is gained when the test particle executes a quarter oscillation. 
where $b$ is the beam pipe radius. For an electron created at a time $t_{0}$ after the head of the bunch passed by, the momentum gain in Eq. 3.12 has to be scaled with a factor $\left(\Delta t_{b}-t_{0}\right) / \Delta t_{b}$.

"Autonomous" electrons gain an energy that depends on the exact period of the oscillation, the time that the particle is subjected to the force, and its energy at the start of the oscillation. The electron oscillation frequency is

$$
\omega_{e}=c \sqrt{\frac{2 \pi Z r_{e} N_{b}}{\sigma_{r}^{2} \sigma_{z}}},
$$

similar to Eq. 2.1. See Table 3.1 for typical RHIC values. For a stationary electron initially at a distance equal to the transverse bunch size $\sigma_{r}$, the maximum energy gain is

$$
\Delta E_{\mathrm{bunch}}=\frac{1}{2} m_{e} \omega_{e}^{2} \sigma_{r}^{2}=\frac{1}{2} m_{e} c^{2} \frac{Z r_{e} N_{b}}{\sigma_{z}}
$$

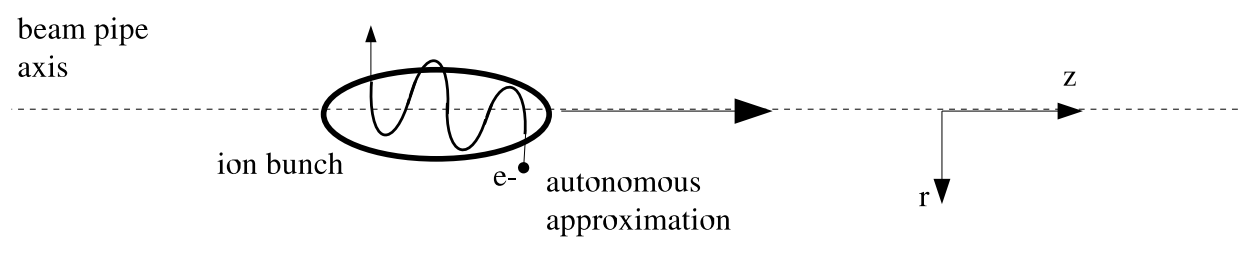

critical radius

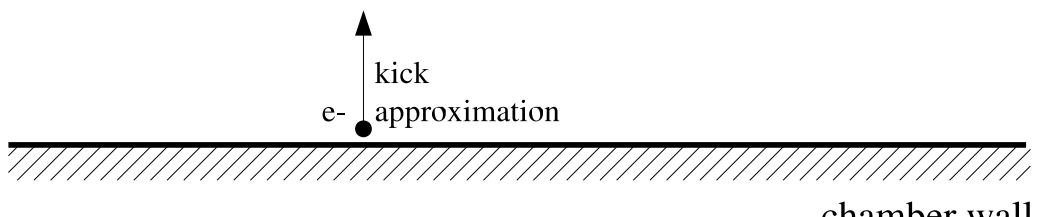

Figure 3.1: Electron-bunch interaction regimes following Berg's formalism in a cylindrical symmetric beam pipe [31].

\subsubsection{Non-uniform bunch charge distribution}

More realistic scenarios consider that:

- the electrons are not stationary, but move during the bunch passage.

- the bunch charge spatial distribution is longitudinally and transversely non-uniform. 
A solution to the first consideration was first derived by O. Gröbner in [26]. Both considerations are included in Ref. [31], whose procedure follows in this section. A longitudinal Gaussian distribution, and three different distributions in the transverse plane are considered: cylindrical, Gaussian, and a parabolic profile expressed by

$$
\lambda_{b}(t)=\left\{\begin{array}{lll}
\lambda_{b}\left[1-\left(\frac{c t}{\sigma_{l}}\right)^{2}\right]^{3} & ; & \text { for }|c t| \leq \sigma_{l} \\
0 & ; & \text { for }|c t| \geq \sigma_{l}
\end{array}\right.
$$

where $2 \sigma_{l}$ is the full bunch length, and $\lambda_{b}$ is the peak line bunch density. Equation 3.16 resembles a Gaussian beam profile smoothly truncated at $c t= \pm \sigma_{l}$ (the equivalent Gaussian rms length is $\sigma_{z} \approx \sigma_{l} / 3$ ).

The longitudinal and transverse distributions are factorised as in Eq. 3.8. The expression for the average energy gain of a uniform distribution of electrons in a circular beam pipe in the autonomous approximation is given by:

$$
\Delta E_{\mathrm{aut}}=m_{e} c^{2}\left(\frac{Z N_{b} r_{e}}{b}\right)^{2} \frac{4}{\pi}\left(\ln \frac{r_{C}}{c_{0} \sigma_{r}}-\frac{1}{2}\right)
$$

where $c_{0}=1.05$ is just a numerical constant [43]. For the kick approximation (outside the radius $r_{C}$ ), the average energy gain is:

$$
\Delta E_{\mathrm{kick}}=m_{e} c^{2}\left(\frac{Z N_{b} r_{e}}{b}\right)^{2} 4 \ln \frac{b}{r_{C}} .
$$

However, Eqs. 3.17 and 3.18 do not always hold for the RHIC case because the critical radius $r_{C}$ becomes comparable to the beam-pipe radius, $b$ (see Table 3.1). Thus, particles initially in the kick regime enter the autonomous regime during the bunch passage. In this case, the average energy deposited into the wall is $[31,43]$

$$
\Delta E_{\mathrm{int}}=m_{e} c^{2} \frac{Z N_{b} r_{e}}{\sqrt{2 \pi} \sigma_{z}}\left(\ln \frac{b}{c_{0} \sigma_{r}}-\frac{1}{2}\right)
$$

Note that Eq. 3.19 includes all the dependencies associated with the bunch parameters. The average energy deposited into the wall increases linearly with the bunch intensity, $N_{b}$, and decreases inversely with the bunch length, $\sigma_{z}$. Although the dependence is weaker due to the logarithmic function, the energy gained increases with the beam pipe radius, $b$, and decreases with the transverse beam size, $\sigma_{r}$.

The energy gained by an electron during a bunch passage depends mainly on its radial position. Figure 3.2 shows the energy gain as a function of its initial radial position for the gold parameters in Table 3.1, considering non-stationary electrons and three transverse bunch distributions: cylindrical, Gaussian, and parabolic. In all cases, the longitudinal distribution is considered to be Gaussian. Note that, consistent with Eq. 3.19, the peaks are a factor of $\sim 4$ larger for store conditions due to the $\sim 4$ times smaller bunch length. No significant differences are found between the Gaussian and parabolic distributions. 
Table 3.1: List of typical RHIC parameters during Run-3, Run-4, Run-5. The average energy gain is computed using Eq. 3.19.

\begin{tabular}{|c|c|c|c|c|c|}
\hline parameter & symbol & unit & $\begin{array}{l}\text { protons }(\mathrm{p}) \\
\text { injection/store }\end{array}$ & $\begin{array}{c}\text { gold }(\mathrm{Au}) \\
\text { injection/store }\end{array}$ & $\begin{array}{l}\text { copper }(\mathrm{Cu}) \\
\text { injection/store }\end{array}$ \\
\hline charge state & $Z$ & - & 1 & 79 & 29 \\
\hline bunch intensity & $N_{b}$ & - & $10^{11}$ & $10^{9}$ & $6 \times 10^{9}$ \\
\hline rms bunch length & $\sigma_{z}$ & $\mathrm{~m}$ & $1.5 / 0.5$ & $1.9 / 0.5$ & $1.9 / 0.5$ \\
\hline rms bunch radius & $\sigma_{r}$ & $\mathrm{~mm}$ & 2 & 2 & 2 \\
\hline chamber radius & $b$ & $\mathrm{~cm}$ & 6 & 6 & 6 \\
\hline critical radius & $r_{C}$ & $\mathrm{~cm}$ & $3.7 / 2.1$ & $3.7 / 2.0$ & $5.5 / 2.8$ \\
\hline electron oscillation freq. & $\omega_{e}$ & $\mathrm{MHz}$ & $90 / 160$ & $75 / 140$ & $100 / 210$ \\
\hline average energy gain & $\Delta E_{\mathrm{int}}$ & $\mathrm{eV}$ & $110 / 330$ & $70 / 260$ & $150 / 570$ \\
\hline
\end{tabular}
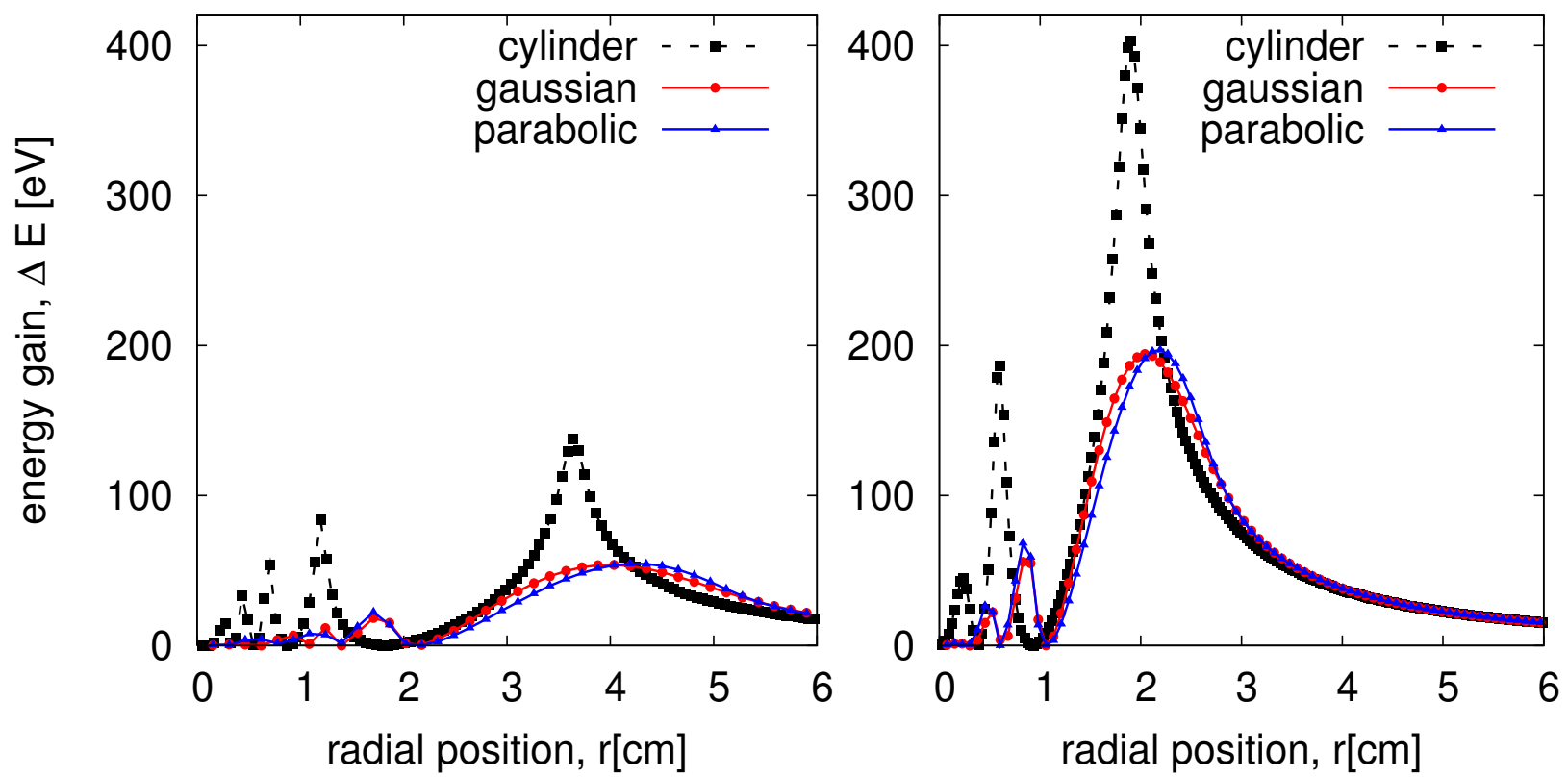

Figure 3.2: Energy gain as a function of the initial radial electron position using three different transverse bunch profiles (cylindrical, Gaussian, and parabolic), applied to the RHIC case under injection (left) and store (right) conditions.

\subsection{Secondary electron yield}

Electron multiplication in the beam-pipe wall is a key ingredient for electron cloud formation. This is parameterized by the Secondary Emission Yield of the chamber surface wall, usually represented by either $\delta$ or SEY, which gives the ratio of emitted secondary electrons per 
incident electron:

$$
\delta=\frac{I_{s}}{I_{p}}
$$

where $I_{s}$ is the measured outgoing electron current (due to secondaries) and $I_{p}$ is the incident electron flux.

Conventionally, three components to the secondary electron flux are defined based on the energy at which the secondaries are emitted [44-46]. The left part in Fig. 3.3 shows a sketch of the secondary emission process, while the right plot shows the contribution of the three components for the case of an incident electron beam of energy $E_{p}=300 \mathrm{eV}$ on a stainless steel surface. The three components are:

1. Elastically backscattered electrons at the wall surface, $I_{\mathrm{el}}$. These electrons are emitted with an energy equal to the energy of the incident particle.

2. True secondaries, $I_{\mathrm{ts}}$, which come from electrons that penetrated few tens of nanometers into the material. Their emission energy is a few $\mathrm{eV}$.

3. Rediffused electrons, $I_{\text {rdf }}$, referring to the electrons that are reflected back after several collisions inside the bulk material. They are emitted at intermediate energies.

The total secondary electron current is the sum of the three components, $I_{s}=I_{\mathrm{ts}}+I_{\mathrm{el}}+I_{\mathrm{rdf}}$, and therefore, $\delta=\delta_{\mathrm{ts}}+\delta_{\mathrm{el}}+\delta_{\mathrm{rdf}}$. However, there is no fundamental distinction between the backscattered and rediffused physical processes. Often no distinction is considered between "rediffused" and "true secondaries" for energies below $\approx 50 \mathrm{eV}$, all electrons being called by the latter name $[43,47]$. The total yield is then taken to be the addition of these two components:

$$
\delta=\delta_{t}+\delta_{r}
$$

where $\delta_{t}$ denotes the yield of true secondaries, and $\delta_{r}$ the reflected yield. This notation is used below unless otherwise stated.

The secondary emission yield is a strong function of the primary electron energy $E$. Several studies have been performed to determine $\delta(E)$ in recent years [48-51]. A typical expression for an electron hitting the wall with normal incidence is based on the Furman and Pivi model [46],

$$
\begin{aligned}
\delta_{r}(E) & =\left(\delta_{0}-\delta_{\infty}\right) e^{-E / E_{r}}+\delta_{\infty}, \\
\delta_{t}(E) & =\delta_{\max }^{*} \frac{s\left(E / E_{\max }\right)}{s-1+\left(E / E_{\max }\right)^{s}}
\end{aligned}
$$

where $\delta_{\max }^{*}$ corresponds to the maximum value of $\delta_{t}(E), E_{\max }$ is the energy at which this maximum takes place, and $\delta_{0}$ corresponds to the backscattering probability for an electron in the energy limit $E \rightarrow 0$. Similarly, the value of $\delta_{\infty}$ corresponds to the backscattering probability for an electron at energy $E \rightarrow \infty$. The parameters $E_{r}$, and $s$ are just fitting parameters. Often, the parameter $\delta_{0}$ is also called reflectivity. Usually, a given surface is characterized by $\delta_{\max }=\delta_{\max }^{*}+\delta_{\infty}$, and $E_{\max }$. 

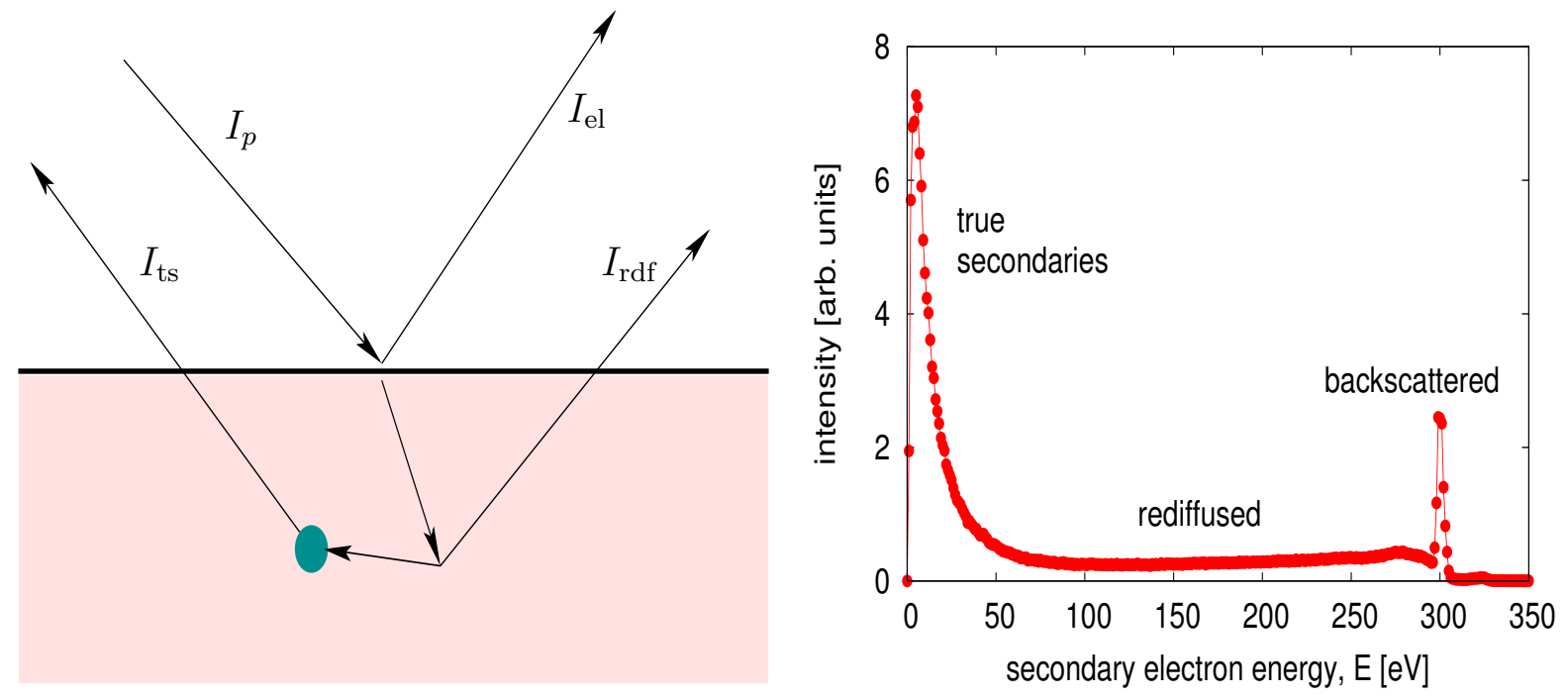

Figure 3.3: Left: sketch showing the different components defining the secondary electron emission following notation in [46]. The blob emphasizes a nontrivial interaction yielding true-secondary electrons. Right: measured energy distribution curves for unbaked and unconditioned stainless steel at normal incidence with $300 \mathrm{eV}$ primary electrons. The distribution shows three different components, corresponding to the true secondaries, rediffused, and elastically reflected electrons $\left(I_{\mathrm{ts}}, I_{\mathrm{rdf}}\right.$, and $I_{\mathrm{el}}$ on the left hand side plot). Data courtesy of R. Kirby and M. Furman [46, 48].

Figure 3.4 depicts the behaviour of the total $\delta(E)$ (red line), and the contributions of the reflected electrons (bold black line) and the true secondaries (dashed line) as described by Eq. 3.22 and 3.23. The blue markers show experimental data for an unbaked and unconditioned stainless steel surface. The fit optimally reproduces the experimental data for

$$
\begin{array}{llrl}
E_{r} & =60 \mathrm{eV}, & s=1.5, & \delta_{\infty}=0.15, \\
\delta_{0}=0.7, & E_{\max }=300 \mathrm{eV}, & \delta_{\max }=1.9 ;
\end{array}
$$

consistent with literature values $[24,46]$.

Since a large fraction of the electrons present in the electron cloud have an energy of a few $\mathrm{eV}$, the value of $\delta_{0}$ becomes a key ingredient (as will be seen in Chapters 6 and 10). Unfortunately, due to technical limitations there is a significant uncertainty in the SEY measurements for low energy electrons $[6,47]$. For instance, the experimental data do not go below $20 \mathrm{eV}$ in Fig. 3.4. This is the main unresolved issue in the secondary emission process. Low energy electrons are not lost when they hit the wall but are reflected back with large probability $\left(\delta_{0}\right.$ ranges between 0.5 and 1 ), hence increasing the electron survival time. Figure 3.5 depicts recent results for the kinetic energy distributions for secondary electrons [47]. As the primary electron energy decreases, the backscattered peak gains more and more relevance.

The SEY also depends on the angle at which the electrons strike the chamber wall. For 


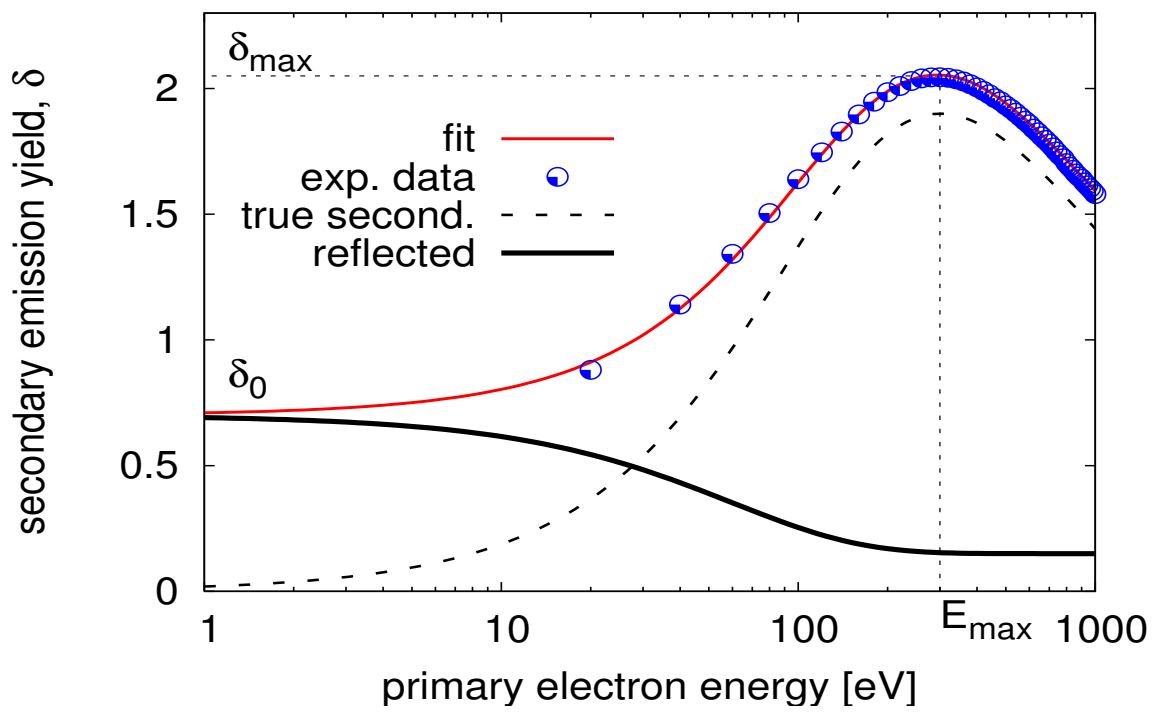

Figure 3.4: Secondary emission yield as a function of the primary incident electron. The blue circles are experimental data provided by R. Kirby, whereas the red line is a fit based on Eqs. 3.22 and 3.23. The dashed line and the bold line correspond to the contribution from true secondaries and reflected electrons, respectively.

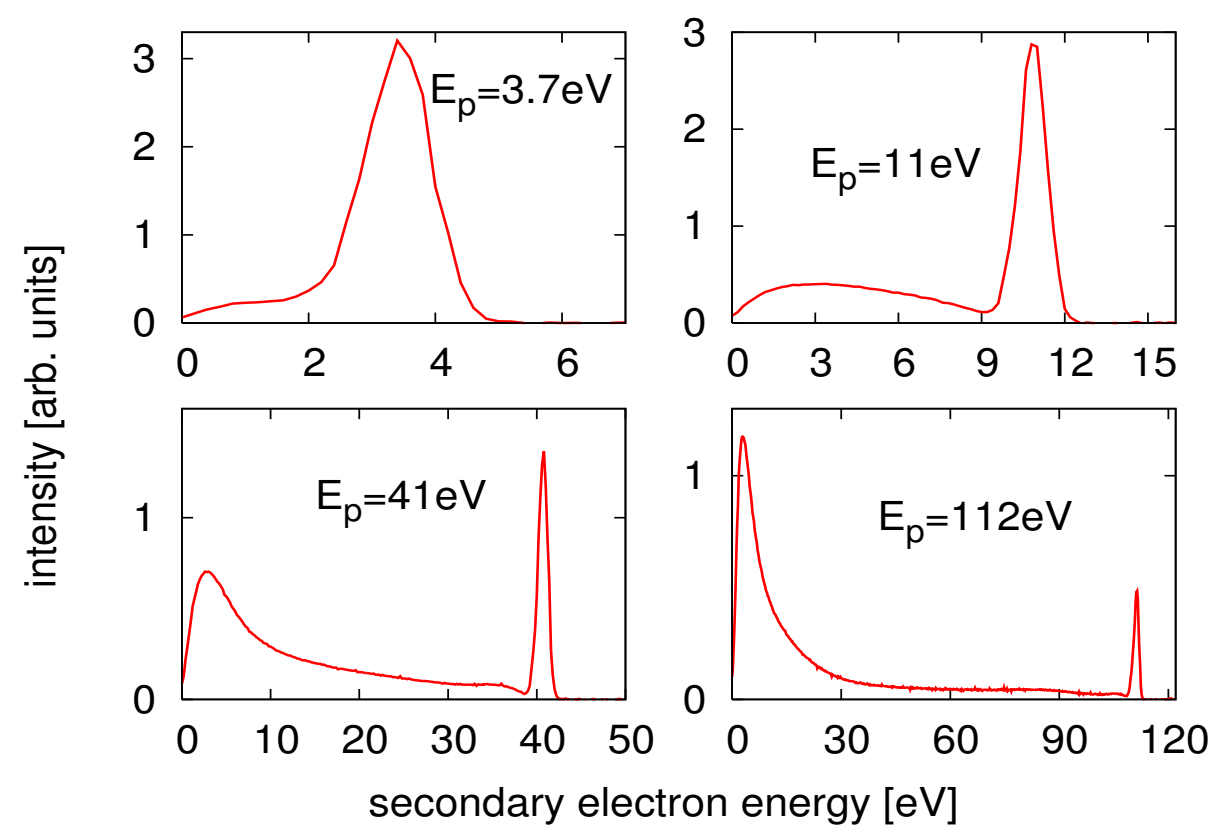

Figure 3.5: Measured energy distribution curves of a fully scrubbed Copper surface for different primary electron energies at normal incidence. Courtesy of R. Cimino and G. Bellodi [47,52]. 
non-normal incidence, the entire $\delta(E)$ in Eq. 3.21 is multiplied by [24]

$$
\delta(E, \theta)=\delta(E) \exp ^{\alpha_{p}(1-\cos \theta)}
$$

where the angle $\theta$ is taken to the surface's normal ( $\theta=0$ indicates perpendicular incidence), and $\alpha_{p} \approx 0.5$ is a fitting parameter $[24,46]$.

\subsection{Saturation effects}

The previous sections showed how the primary electrons are created (Section 3.1), accelerated to the beam pipe due to the bunch interaction (Section 3.2), and multiplicated as a function of the chamber SEY (Section 3.3). However, the electron multiplication is not unlimited. The electron cloud build up saturates when the electron losses balance the electron generation rate [2]. Depending on the bunch charge, $N_{b}$ and if multipacting occurs, this can be (roughly) estimated for two regimes [53]:

1. For low bunch charges, saturation takes place when the average neutralization density is reached, i.e. average electric field on the wall is zero. This leads to a linear electron density of

$$
\lambda_{\text {sat }}=N_{b} / s_{b},
$$

where $s_{b}$ is the distance between bunches ${ }^{\dagger}$.

2. For large bunch charges, saturation occurs when the energy at which the secondary electrons are emitted, $\left\langle E^{\prime}\right\rangle$ is not large enough to penetrate into the space charge field of the cloud. In this case, the linear saturated density is [53]

$$
\lambda_{\mathrm{sat}}=\frac{\pi\left\langle E^{\prime}\right\rangle}{m_{e} c^{2} r_{e}} .
$$

Note that the first regime shows a direct linear dependence on the bunch population, $N_{b}$, while the second does not. The transition occurs at [53]

$$
N_{\text {trans }}=\frac{\left\langle E^{\prime}\right\rangle s_{b}}{m_{e} c^{2} r_{e}} .
$$

References $[2,53]$ consider $\left\langle E^{\prime}\right\rangle=E_{\text {sec }}$, independent of the bunch intensity, bunch spacing, et cetera. Thus, the conclusion is that if multipacting occurs, the saturated electron density first increases with the bunch population, until it reaches the transition value given by Eq. 3.27, above which it stays approximately constant. For RHIC, with bunch spacings $s_{b}=32 \mathrm{~m}(108 \mathrm{~ns})$ and considering a $\left\langle E^{\prime}\right\rangle=E_{\mathrm{sec}}=8 \mathrm{eV}$, this transition occurs at $N_{\text {trans }}=1.7 \times 10^{11}$ protons $/$ bunch.

\footnotetext{
${ }^{\dagger}$ Note that this is a very crude approximation, since no dependence is shown with the SEY, beam pipe radius, et cetera
} 


\subsection{Electron cloud computer simulation codes}

The electron cloud ingredients discussed in the previous sections do not lead to an analytical (nor trivial) prediction whether multipacting occurs or not. Several computer codes have been (and still are being) successfully developed over the last years to simulate an electron cloud build-up for almost arbitrary conditions, i.e. beam parameters, beam pipe geometries, beam pipe wall surface properties, et cetera. The final result is sensitive to small changes in one parameter [8]. A comparison among the different codes is shown at Ref. [54]. For this thesis, I mostly used CSEC (Cylindrically Symmetric Electron Cloud), originally written by M. Blaskiewicz for studies at the PSR. First descriptions of the code can be found in $[8,24]$.

\subsubsection{Cylindrical Symmetric Electron Cloud, CSEC}

CSEC assumes an electron cloud that is cylindrically symmetric within a round, straight vacuum chamber. The source of primary electrons is introduced externally: the user can choose the number of primary electrons and their initial location, either at the wall (from beam lost particles striking the wall at grazing incident angles), or in the beam core (from beam gas ionization). This generation rate must be estimated by the user, for example from Eqs. 3.1 and 3.2, respectively.

The spatial distribution of the electron cloud is modeled as the sum of macro cylindrical concentric shells serving as macroparticles or macroelectrons, whose charge is much larger than that of a single electron. The CSEC user sets a minimum and a maximum for the macroparticle charge in the input file, according to a balance between the accuracy and the required CPU time. The macroparticle shells can have an angular momentum to take into account non perpendicular electron-wall collisions. A machine turn is divided into different time steps, or number of slices per turn. This number has to be carefully chosen to optimally reproduce the (macro)electron motion inside the bunch fields. In addition to the beam bunch fields, the electron space-charge field is also taken into account. Since CSEC assumes a cylindrical symmetry, the beam and macroelectron image charges are neglected. This is not legitimate if one wishes to study electron clouds inside dipole fields, offset beams, or non-circular vacuum chambers.

Analogous to Eq. 3.10, at each time step CSEC computes the acceleration of the macroparticle $j$, with radius $r_{j}$ due to the shell $k$ by [8]

$$
\ddot{r}_{j}=2 r_{e} c^{2} \lambda_{k} \frac{r_{j}}{d^{2}+r_{j}^{2}}
$$

where $\lambda_{k}$ is the electron line density of shell $k$, and $d$ is a "smoothing length" to avoid divergences at $r=0$. This length is typically slightly smaller than the beam size. The electric field due to the ion beam has a similar form, replacing $\lambda_{k}$ by the bunch density, which follows a profile given by Eq. 3.16.

Whenever a macroparticle hits the wall, it is reemitted as either elastically reflected or true secondary (Eqs. 3.22 and 3.23), and its charge is changed according to the value of the SEY, computed as a function of its angle and energy. As shown in Fig. 3.3, right, 


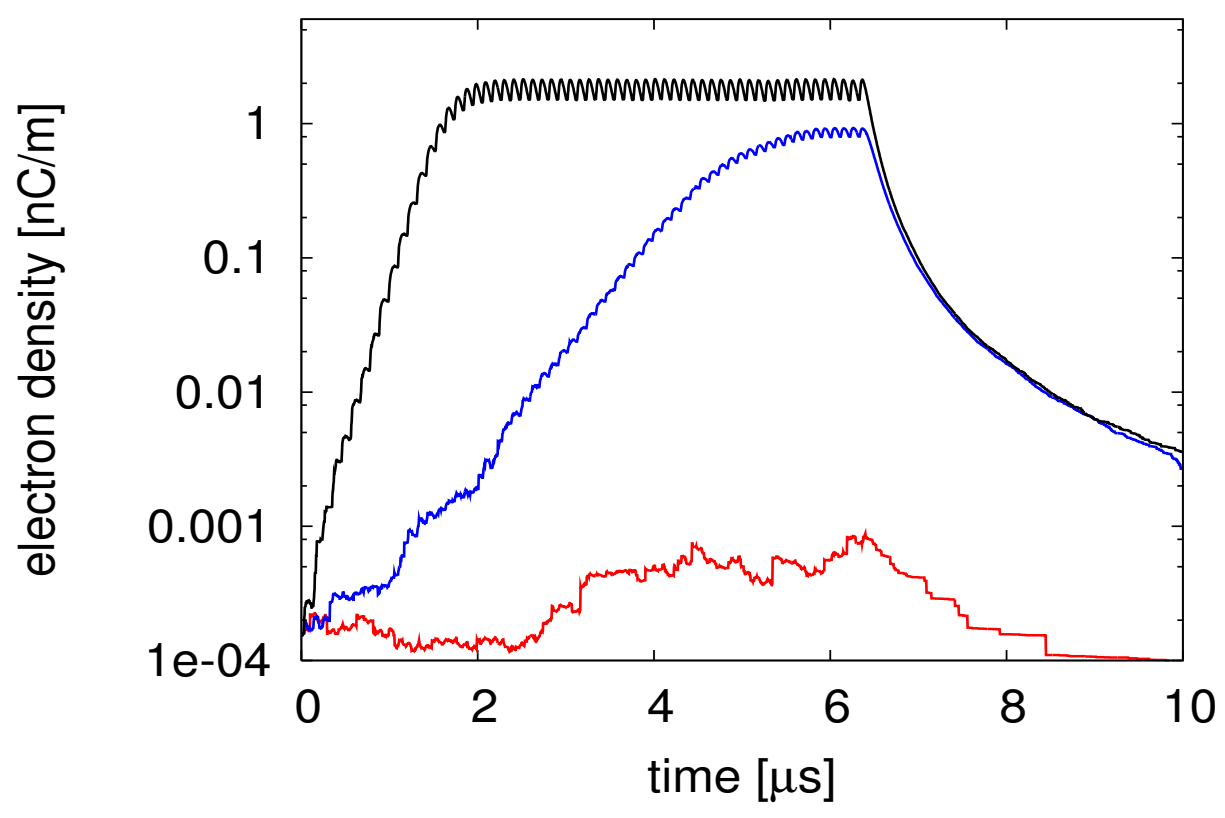

Figure 3.6: Simulated electron cloud build up using CSEC for three different bunch intensities, $N_{b}=4$ (bottom trace, in red), $N_{b}=8$ (middle trace, in blue), and $N_{b}=12 \times 10^{10}$ protons $/$ bunch (top trace, in black). For all cases, a bunch pattern of 60 consecutive bunches spaced by $107 \mathrm{~ns}$ is used. For the lowest bunch charge, the electron cloud does not build up, for $N_{b}=8$, and $12 \times 10^{10}$ protons/bunch the electron density grows exponentially $\sim 5$ orders of magnitude until it reaches a saturated value.

for a (primary) macroparticle hitting the wall with energy $E_{0}$, the energy of the emitted (secondary) macroparticles, $E^{\prime}$, depends on whether it is elastically reflected $\left(E^{\prime}=E_{0}\right)$, rediffused (at intermediate energies), or true secondary $\left(E^{\prime} \approx 8 \mathrm{eV}\right)$. In the latter case, the energy distribution follows a Lorentzian form centered at $E_{\mathrm{sec}}$ and width $\sigma_{\mathrm{sec}}$. Typical values for stainless steel are $E_{\mathrm{sec}} \approx 8 \mathrm{eV}$, and $\sigma_{\mathrm{sec}} \approx 4.5 \mathrm{eV}[46]$.

\subsubsection{Example for RHIC}

Figure 3.6 shows three different electron cloud evolution simulations with three different bunch intensities, $N_{b}=4,8$, and $12 \times 10^{10}$ protons/bunch. Table 3.2 contains the physical parameters, used as input parameters for CSEC. The electron density starts with approximately $2 \times 10^{-4} \mathrm{nC} / \mathrm{m}$, which is set as an input parameter in CSEC (parameter $\rho_{c e}$ in Table 3.2). For a bunch population of $4 \times 10^{10}$ protons/bunch, the electron density does not build up from turn to turn.

For $N_{b}=8 \times 10^{10}$ protons/bunch, an exponential build up is observed until space charge effects limit the growth, and a saturated value is achieved at approximately $0.7 \mathrm{nC} / \mathrm{m}$ after $6 \mu \mathrm{s}$. The growth time is shorter for $N_{b}=12 \times 10^{10}$ protons/bunch, and the saturated value is larger (approximately $1.5 \mathrm{nC} / \mathrm{m}$ ). Note that the saturated values are of the same 
order of magnitude as predicted by Eq. 3.25, yet the ratio between saturated values does not correspond to the ratio between bunch intensities,

$$
0.7 / 1.5 \neq 8 \times 10^{10} / 12 \times 10^{10}
$$

as predicted by Eq. 3.25. This is not a contradiction, since the electron cloud build up is sensitive to many parameters not included in Eq. 3.25, which is only an approximation.

For $N_{b}=12 \times 10^{10}$ protons/bunch, the non-straight decay curve in Fig. 3.6 (note the logarithmic vertical scale) for $t>6 \mu$ s indicates that the decay is not governed by a single exponential regime. Typically, the decay is governed by two regimes [55]: in the first, the electron density decays rapidly due to the cloud temperature (a consequence of the space charge, which repels electrons towards the vacuum chamber). In the second (for weak space charge forces), the electrons bounce back and forth across the chamber with low energies $(\sim 5 \mathrm{eV})$, so the decay is mainly dominated by the reflectivity of the surface chamber (parameter $\delta_{0}$ in Eq. 3.22) and the chamber radius (see Appendix B).

Table 3.2: List of input parameters for electron cloud simulations in Fig. 3.6.

\begin{tabular}{|c|c|c|c|}
\hline parameter & symbol & unit & value \\
\hline bunch spacing & $s_{b}$ & ns & 107 \\
\hline number of bunches & $\ldots$ & $\ldots$ & 60 \\
\hline full bunch length & $\sigma_{l}$ & ns & 15 \\
\hline bunch shape parameter & $n$ & $\ldots$ & 3 \\
\hline bunch population & $N_{b}$ & $10^{10}$ protons & $4 / 8 / 12$ \\
\hline rms beam radius & $\sigma_{r}$ & $\mathrm{~mm}$ & 2.4 \\
\hline pipe radius & $b$ & $\mathrm{~mm}$ & 60 \\
\hline initial electron density & $\rho_{c e}$ & $\mathrm{pC} \cdot \mathrm{m}^{-1}$ & 0.2 \\
\hline electrons generated/bunch & $\ldots$ & $\ldots$ & 20000 \\
\hline electron generation radius & $\ldots$ & $\mathrm{mm}$ & 60 \\
\hline longitudinal slices per turn & $\cdots$ & $\cdots$ & 108000 \\
\hline macro-particles, initially & $\ldots$ & $\ldots$ & 25 \\
\hline smoothing length & $d$ & $\mathrm{~mm}$ & 1.0 \\
\hline reflection probability for $E \rightarrow 0$ & $\delta_{0}$ & $\ldots$ & 0.6 \\
\hline reflection probability for $E \rightarrow \infty$ & $\delta_{\infty}$ & $\ldots$ & 0.2 \\
\hline maximum SEY & $\delta_{\max }$ & $\cdots$ & 2.2 \\
\hline energy at maximum SEY & $E_{\max }$ & $\mathrm{eV}$ & 310 \\
\hline reflection energy & $E_{r}$ & $\mathrm{eV}$ & 60 \\
\hline energy of secondary emitted electrons & $E_{\mathrm{sec}}$ & $\mathrm{eV}$ & 8.9 \\
\hline distribution width of secondary electrons & $\sigma_{\mathrm{sec}}$ & $\mathrm{eV}$ & 4.5 \\
\hline SEY exponent & $s$ & $\ldots$ & 1.83 \\
\hline angular parameter & $\alpha_{p}$ & $\ldots$ & 0.5 \\
\hline
\end{tabular}




\subsubsection{Average energy at wall impact}

Figure 3.7 illustrates the average energy at which the electrons strike the chamber wall between two bunch passages, using CSEC and the input parameters listed in Table 3.2 for two different bunch intensities in RHIC: $N=4 \times 10^{10}$ (red circles) and $N=12 \times 10^{10}$ protons (blue squares). Electrons receive a boost during the bunch passage (black trace), but the (average) energy decreases almost exponentially until the next bunch arrives. The right hand plot in Fig. 3.7 shows the corresponding SEY (or $\delta$ ) for the average energy depicted in Fig. 3.7 (left). For $N=4 \times 10^{10}, \delta<1$ and no electron cloud builds up (as seen in Fig. 3.6). For $N=12 \times 10^{10}, \delta>1$ when the electrons receive the bunch's acceleration, but it then decreases exponentially, and the electron cloud consistently builds up (as seen in Fig. 3.6).
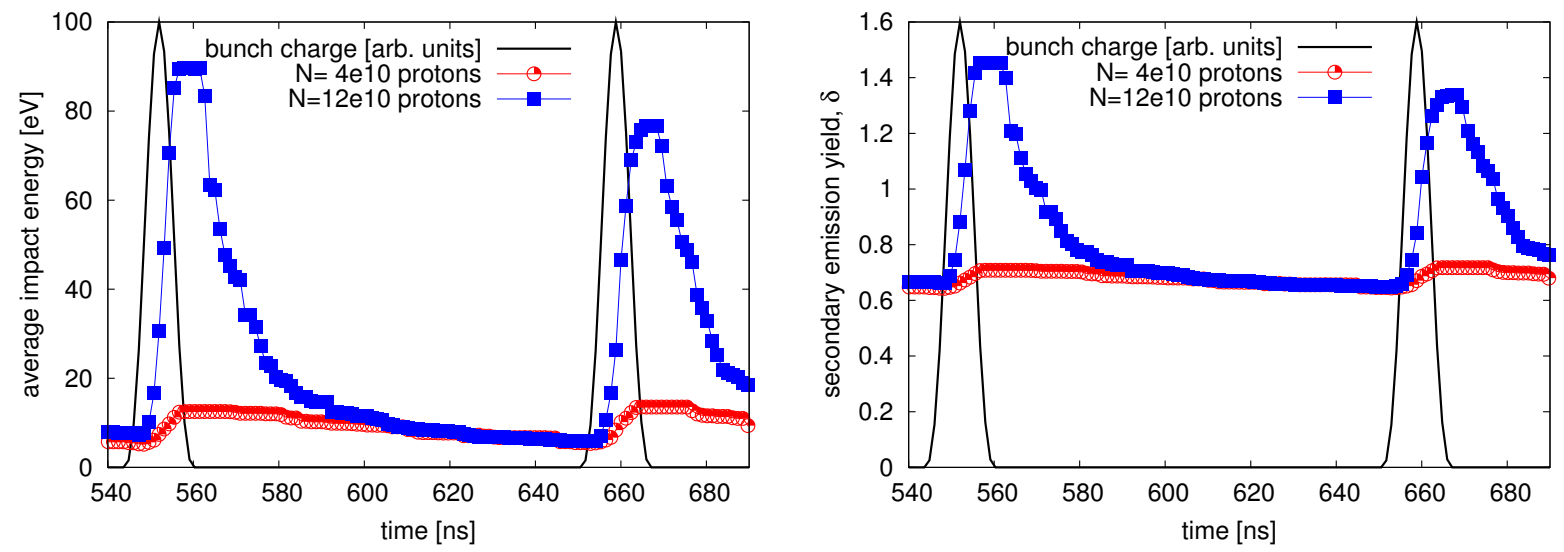

Figure 3.7: Energy at which the electrons strike the wall between two bunch passages (left) and the corresponding SEY (right). Electrons receive a boost from the bunch passage, and they rapidly dissipate into a slow motion. 


\section{PART II}

\section{EXPERIMENTAL OBSERVATIONS}





\section{Chapter 4}

\section{The RHIC electron detector}

One of the main concerns of this work is to obtain a proper diagnostics for the pressure rises limiting machine operation since Run-2 (2001/2002). Since the first analysis showed the pressure rises are compatible with molecular desorption induced by electron multipacting, a first priority was to install devices able to detect these electrons. Before Run-3, up to 15 electron detectors (ED) were installed in the RHIC ring, 11 of them designed "ad hoc" for RHIC. This Chapter describes the RHIC electron detector.

\subsection{A Retarding Field Analyzer}

The working principle of the detector is shown in Fig. 4.1. This detector is often called Retarding Field Analyzer (RFA) because it is able to analyze the energy of the multipacting electrons by means of an retarding electrostatic field. The RHIC ED is based on the first PSR design [56], although similar detectors have also been installed in other machines, like APS [57], SPS [58] and BEPC [59]. In RHIC, the RFA is mounted on a $12 \mathrm{~cm}$ inner diameter tee, perpedincular to the vacuum pipe.

The top grid ("Grid 0" in Fig. 4.1) is welded to the beam pipe and acts as an RF shield. Its transparency $\left(T_{0}\right)$ is fixed to $23 \%$ in order to decrease the effect of the image currents (see Section 4.4) without interfering with the multipacting process.

The middle grid ("Grid 1" in Fig. 4.1, with $T_{1}=80 \%$ ) can be biased to different voltages $\left(V_{\text {grid }}\right)$ acting as an electron energy filter; electrons with energy lower than $\left|e V_{\text {grid }}\right|$ cannot traverse Grid 1 and are not collected. This is why it is also called the "filter grid".

The bottom grid ("Grid 2" in Fig. 4.1, with $T_{2}=80 \%$ ) is held at $-10 \mathrm{~V}$ by a DC battery. As shown in Fig. 4.1, it was originally conceived to repel back the secondary electrons produced at the collector. However, after the installation of the electron detectors, a larger signal is observed when this grid is grounded. Thus, this grid is grounded unless otherwise stated.

The collector can be biased either positively or negatively $\left(V_{\text {col }}\right)$ to check the presence of either electrons or ions (although it is very hard to detect ions due to their large inertia). The signal produced by the electrons hitting the collector is finally carried to the data acquisition system. 

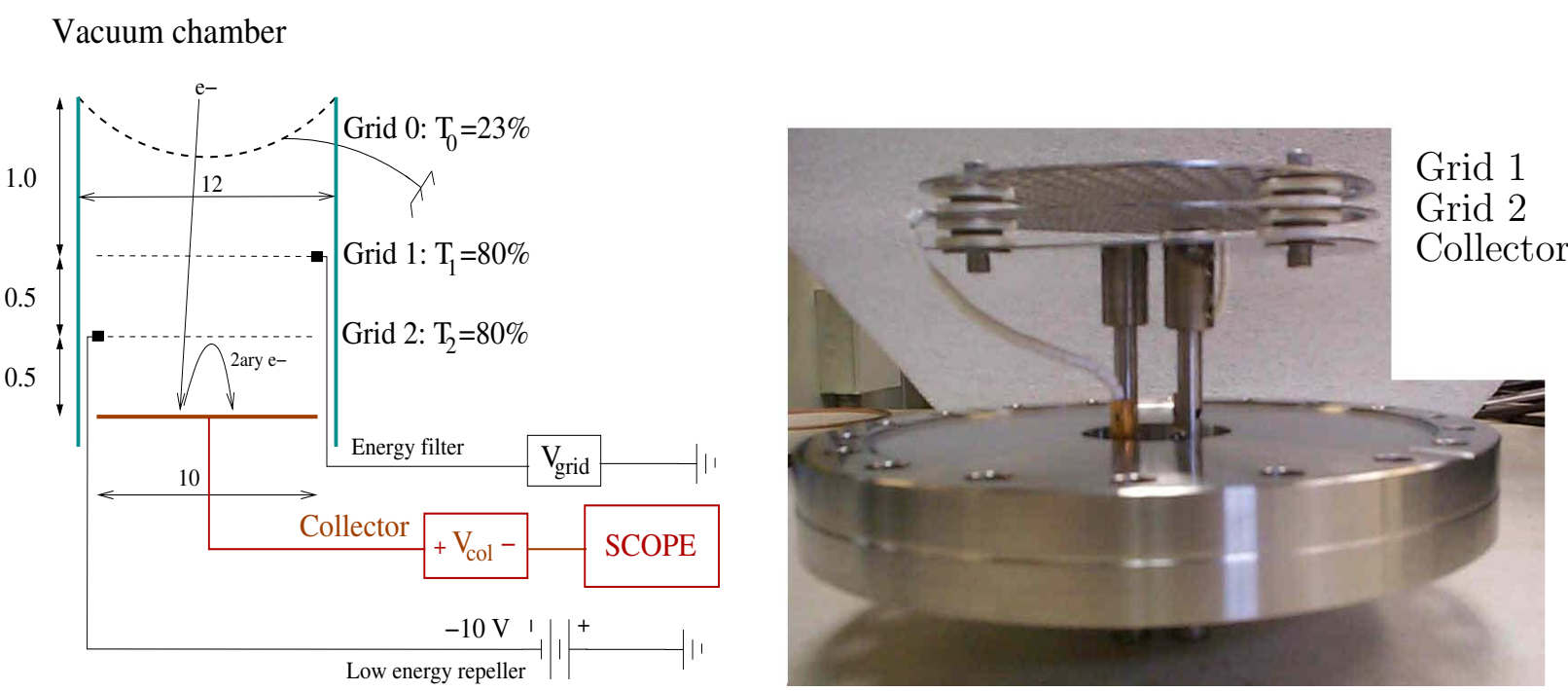

Figure 4.1: RHIC electron detector layout (left) and photograph (right). On the left, the layout shows a front view and the beam travels in the vacuum chamber in the direction perpendicular to the paper. Distances are given in $\mathrm{cm}$ and are not to scale.

\subsection{Data acquisition and control system}

The system for control and data acquisition is shown in Fig. 4.2. The ED is AC coupled to the amplifier Sonoma 310, with a gain $\mathrm{G}=32 \mathrm{~dB}$, and a bandwidth of $[10 \mathrm{kHz}-1 \mathrm{GHz}]$. Due to the small signals detected, two amplifiers in series were usually connected for a total gain of $\mathrm{G}=64 \mathrm{~dB}$. The final low frequency cut-off of the system is determined by the $R L C$ circuit, designed and implemented $a d$ hoc by the C-AD Instrumentation group* to protect the amplifier when the collector is biased to different DC voltages. The value of the capacitor, $C$, is not the same for all the electron detectors, thus the low frequency cut-off changes (see Appendix A). Some of the electron detectors were equipped with neither the $R L C$ circuit nor the amplifier for testing purposes. The electronic changes at the EDs are updated through the web at [60].

The electron detectors are installed in RHIC Sectors 12 and 2. Currently RHIC has 16 electron detectors in total, but only two scopes are allocated for data readout, each scope with four channels. This means that only the signals from 8 electron detectors can be logged at the same time. One scope is for the electron detectors at sector 12, the second scope is for the electron detectors at sector 2. The choice of a given ED is made using the radio frequency multiplexer (denoted as "MUX" in Fig. 4.2). The Multiplex Analog to Digital Converter card (MADC in Fig. 4.2), is used to obtain the electron signal with a sampling rate of $720 \mathrm{~Hz}$. The power supply is used to polarize "grid 1" at different voltages and to allow the measurement of an energy spectrum.

* Many thanks to D. Gassner and J. Gullotta 


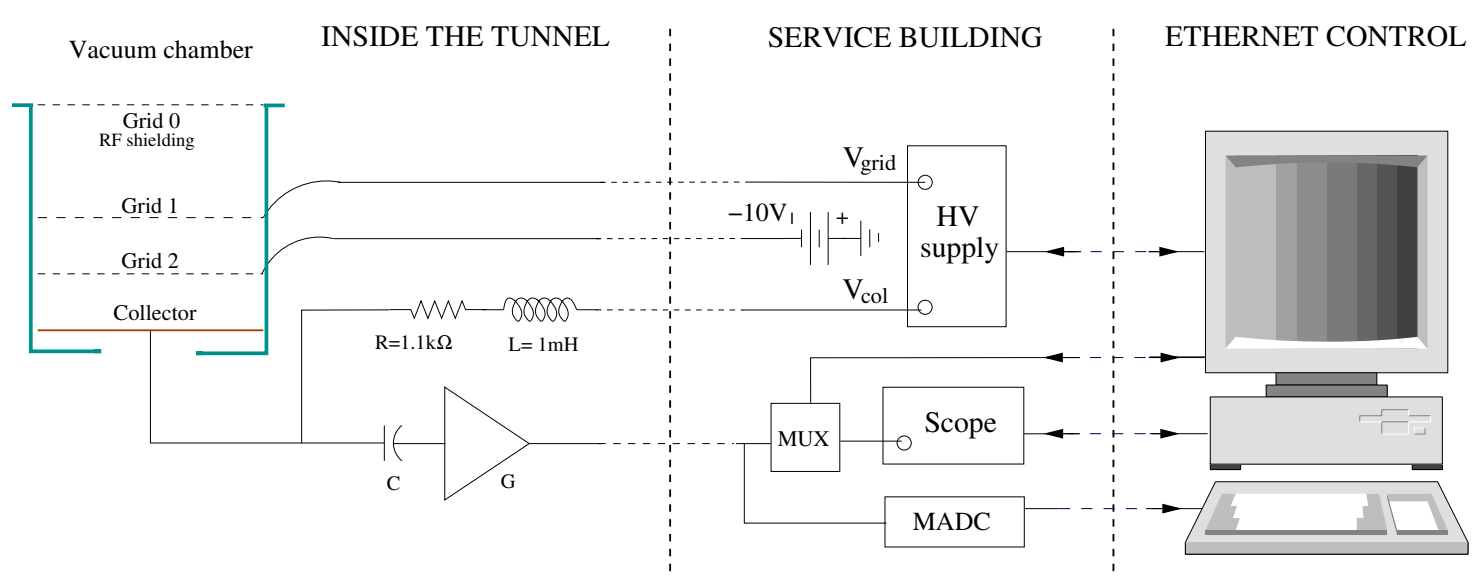

Figure 4.2: RHIC electron detector layout. Part of the electron flux hitting the chamber wall arrives at the collector plate. The signal is then magnified using the amplifier (marked with a $G$ ) and transmitted to the scope. "Grid 1" is used to filter the energy and bias the collector plate using the High Voltage supply. The capacitor between the collector plate and the amplifier protects the latter device from DC current coming from the bias voltage. The capacitor is not the same for all EDs, but a typical value is $\mathrm{C}=100 \mathrm{nF}$.

\subsection{Multipacting signal in the electron detector}

The ED is AC coupled to the system electronics (see Fig. 4.2). An AC coupled system differentiates the signals below the low frequency cut-off, $f_{\text {low }}$, which is determined by the capacitor $C$ for each ED. Typical values for these capacitors are $C=100 \mathrm{nF}$ and $10 \mathrm{nF}$, from which the frequency cut-off is about $f_{\text {low }}=30 \mathrm{kHz}$ and $300 \mathrm{kHz}$, respectively - see Appendix A.

The ED electronics has been modelled using commercial electronic software PSPICE [61]. The analogous circuit is shown in Fig. 4.3 without the amplifier, which only represents a multiplication factor. For simplicity, the circuit model assumes an impedance source $Z_{\text {source }} \rightarrow \infty$, and no parasitic resistances. To study how the multipacting signal is affected by the system electronics, the simulated bunch to bunch electron flux using CSEC ( $\left.I_{\mathrm{CSEC}}\right)$ is introduced as the current source in Fig. 4.3. The output of interest is the voltage that decays in the scope resistor ( $V_{\text {scope }}$ in Fig. 4.3).

The result is shown in Fig. 4.4: the top plot shows the evolution of $I_{\mathrm{CSEC}}$ during two turns, the bottom plot shows $V_{\text {scope }}$ during the same period. The DC part of the signal is lost, so the result is a signal that averages null over its period due to the AC coupling. Note that the the build up and decay times are not affected by the system electronics, as it corresponds to frequencies much larger than $f_{\text {low }}$. The multipacting build up frequency in RHIC is typically around $1 \mathrm{MHz}$, the multipacting decay frequency is around $5 \mathrm{MHz}$. This is consistent with the high pass filter analysis in Appendix A. Secondly, note that the peak to peak signal ratio is not attenuated by the system electronics and follows Ohm's law:

$$
V_{\mathrm{scope}}^{p p}=Z_{\mathrm{scope}} \times I_{\mathrm{CSEC}}^{p}=50 \times 0.8=40 \mathrm{~V} .
$$




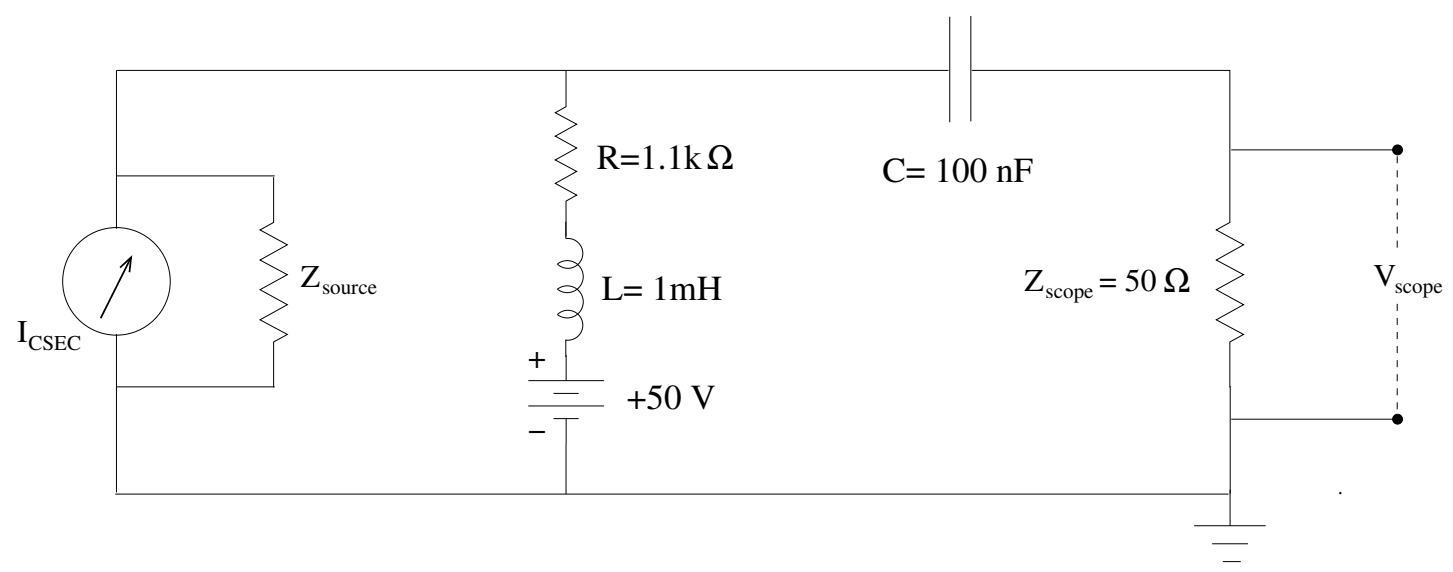

Figure 4.3: Electronic circuit representation to study the ED response to a multipacting signal. The multipacting signal is introduced as a current source that is given by the computer simulation code CSEC. The output under study is the voltage in the scope resistor $\left(V_{\text {scope }}\right)$.

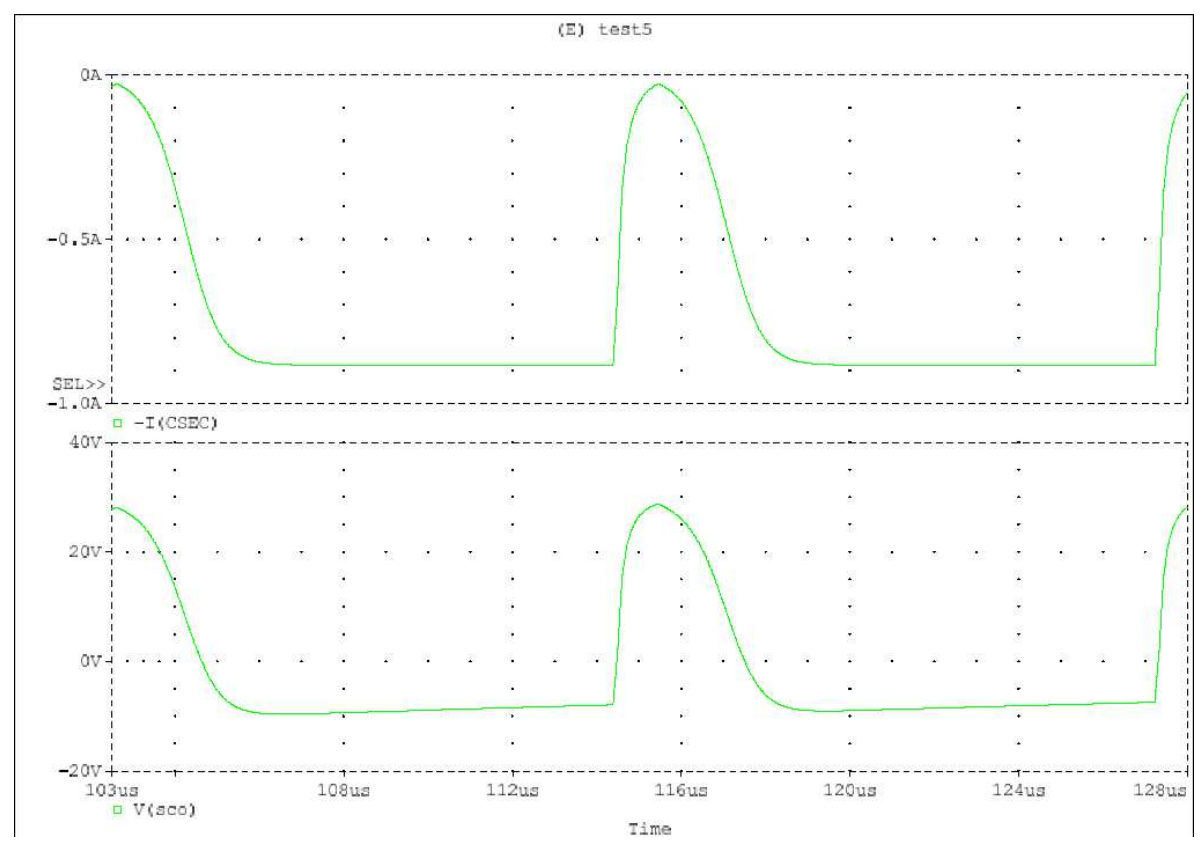

Figure 4.4: Simulated electron signal at the collector using a CSEC input (top plot), and the corresponding scope voltage according to the PSPICE simulations. The peak to peak signal is maintained and follows Ohm's law for the equivalent circuit shown in Fig. 4.3.

\subsubsection{MADC data acquisition system}

As seen in Fig. 4.2, the MADC card is placed before the Multiplexer that is used to select the signal on the scope. The MADC card takes 720 samples/second and sends them to the ethernet network. By logging the maximum and minimum values of these 720 samples 
each second one can qualitatively follow the electron cloud evolution at $1 \mathrm{~Hz}$ for any ED, even those that are not connected to the scope. The MADC acquisition system is used for applications where just the relative values are important, for instance, the measurement of the energy spectrum. Figure 4.5 compares the signal maximum and minimum logged every second $(1 \mathrm{~Hz})$ using the MADC (inner traces) with the maximum and minimum obtained using the scope (outer points). Even though the more reliable signal from the scope is larger than the one registered by the MADC, one can see that they exhibit similar behaviour.

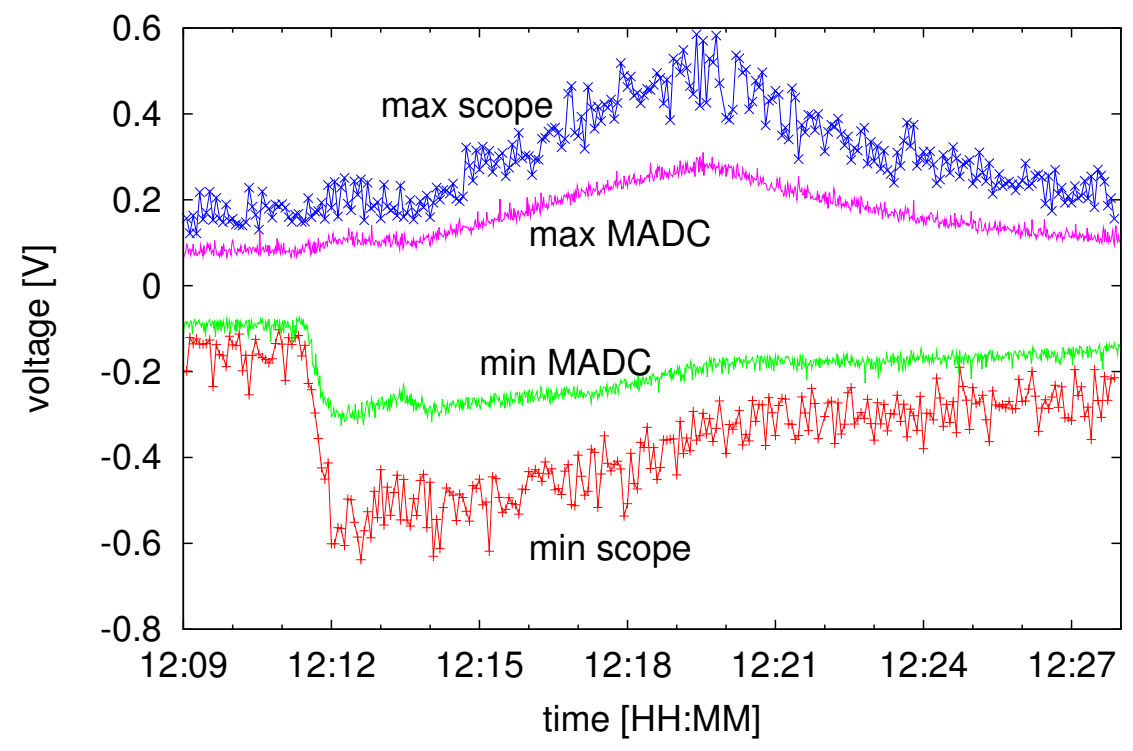

Figure 4.5: Maximum and minimum signals obtained using the scope (top and bottom traces) compared with the maximum and minimum obtained using the MADC (inner traces). Although the values are different, they exhibit a similar behaviour.

\subsection{Image currents in the electron detector}

One of the main noise sources in the ED are the image currents produced by the beam bunch. The ED can be considered as a button pickup electrode, similar to the ones used for Beam Position Monitoring (BPM) [62]. The 23\% transparency grid ("Grid 0" in Fig. 4.1) designed for the RF shielding attenuates the signal, but it does not totally suppress it.

For a bunch charge $e N$ in a Gaussian profile with rms bunch length $\sigma_{z}$, the voltage seen by the button pick up is [62]:

$$
V(t)=\frac{e N}{\sqrt{2 \pi}} \frac{\pi r^{2} Z}{2 b \beta c} \frac{t}{\sigma_{z}^{3}} e^{-\frac{t^{2}}{2 \sigma_{z}^{2}}},
$$

where the time origin is conveniently chosen at the maximum of the bunch Gaussian distribution. Note that Eq. 4.2 is basically a doublet (derivative from a Gaussian profile), with 

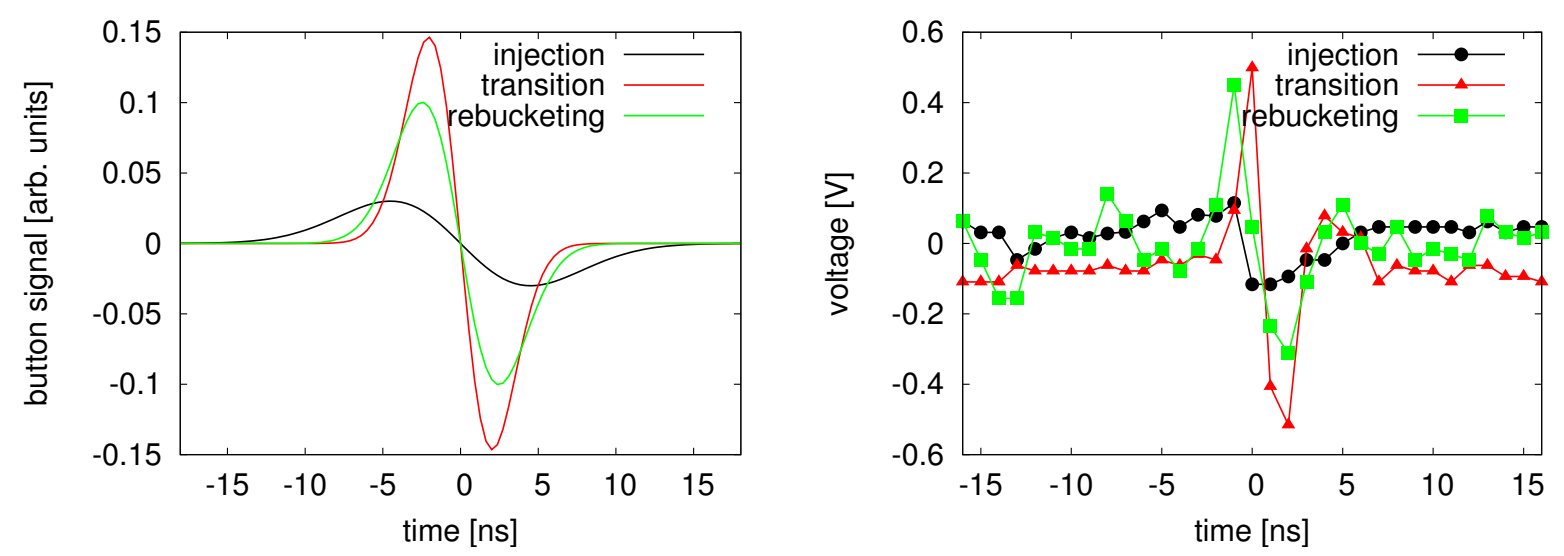

Figure 4.6: Left: Theoretical doublet seen by a button pick up for the three different bunch lengths in Table 4.1. Right: snapshot produced by a bunch passage at three different beam conditions (injection, transition, store).

maxima at $t= \pm \sigma_{z}$, whose values depend linearly on the bunch charge population $N$ and inversely proportional to the square of the bunch length, $\sigma_{z}^{2}$. That is to say, the peak to peak voltage produced by a Gaussian beam in this pick up button scales like:

$$
V_{\mathrm{pp}} \propto \frac{N}{\sigma_{z}^{2} \beta} .
$$

The right hand side plot in Fig. 4.6 shows the experimental data (1 sample/ns) taken with the electron detector at injection, two seconds before transition, and after rebucketing conditions during Fill \#2760. Although the ED has a shield to attenuate the image currents effect, Fig. 4.6 (right) shows that the signal ratio given by Eq. 4.3 is conserved within a reasonable error range. It is worth mentioning that when the beams cross the transition energy the rms bunch length is measured to be as short as $1.5 \mathrm{~ns}$ [63] (instead of the $2.1 \mathrm{~ns}$ in Fig. 4.6). Since the longest bunch length is $\approx 20 \mathrm{~ns}$, this source of noise is strongly noticeable for frequencies above $50 \mathrm{MHz}$.

Table 4.1: Average rms length for deuteron fill \# 2760 at injection, close to transition crossing, and after rebucketing conditions, and corresponding peak to peak signal in the ED at BO2. The

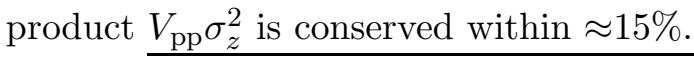

\begin{tabular}{lccc}
\hline \hline conditions & rms bunch length & peak-to-peak ED signal & product \\
$\ldots$ & $\sigma_{z}(\mathrm{~ns})$ & $V_{\mathrm{pp}}(V)$ & $V_{\mathrm{pp}} \sigma_{z}^{2}\left(\mathrm{Vns}^{2}\right)$ \\
\hline injection & 4.5 & 0.23 & 4.7 \\
transition & 2.1 & 1.01 & 4.4 \\
rebucketing & 2.5 & 0.79 & 4.8 \\
\hline \hline
\end{tabular}

Table 4.1 lists the average rms bunch length $\sigma_{z}$ measured by the Wall Current Monitor (WCM), the peak to peak ED signal, $V_{\mathrm{pp}}$, and the product $V_{\mathrm{pp}} \sigma_{z}^{2}$. This product follows 
Eq. 4.3 within an error bar of $\approx 15 \%$. Equation 4.2 has been represented in Fig. 4.6 (left) for a fixed bunch charge and the three different bunch lengths listed in Table 4.1 (fill \#2760).

Equation 4.2 refers to a centered bunch in the vacuum chamber. As the beams are accelerated and cross the transition energy, the beam orbit suffers significant offset displacements, and the doublets in Fig. 4.6 can be significantly distorted.

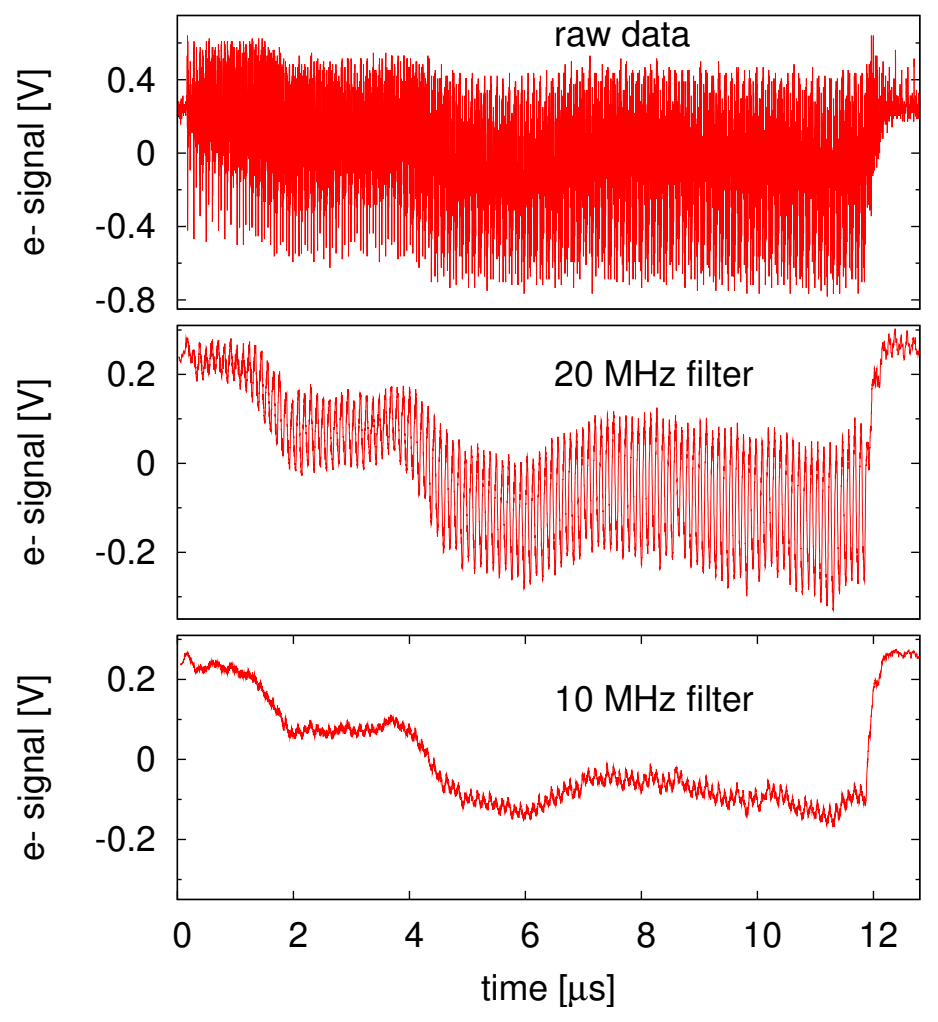

Figure 4.7: ED signal using the scope. The raw data (top plot) do not show any clear signal, but after applying a $20 \mathrm{MHz}$ (middle plot) and $10 \mathrm{MHz}$ filter (bottom plot), the build up and decay of an electron cloud is visible.

\subsection{Experimental examples}

1. A typical electron signal including the effect of the image currents is shown in Fig. 4.7. The raw data is sampled at $1 \mathrm{GS} / \mathrm{s}$ and is shown in the top plot. It is shown in the middle plot after a numerical $20 \mathrm{MHz}$ smoothing filter. Finally, the same data is shown in the bottom plot after a numerical $10 \mathrm{MHz}$ smoothing filter. Note that it is very hard to distinguish any electron cloud formation using the raw data (1 sample/ns) due to the noise produced by image currents, reflections in the cable, et cetera. Instead, by smoothing the data with a linear moving average $20 \mathrm{MHz}$ filter, the signal becomes more clear and one can distinguish the build up and decay of the electron cloud, and can even see oscillations due to the bunch passages. By applying a $10 \mathrm{MHz}$ filter, this 
oscillation is lost, but the information on how the signal builds up and decays, as well as the value at saturation is maintained. This is (partially) overcome using the $20 \mathrm{MHz}$ filter option in the scope. Nonetheless, often a numerical $10 \mathrm{MHz}$ filter is used to get rid of all the noise due to frequencies above those correspoding to the shortest bunch spacing (recall from Table 1.3 that this is $107 \mathrm{~ns}$ ).

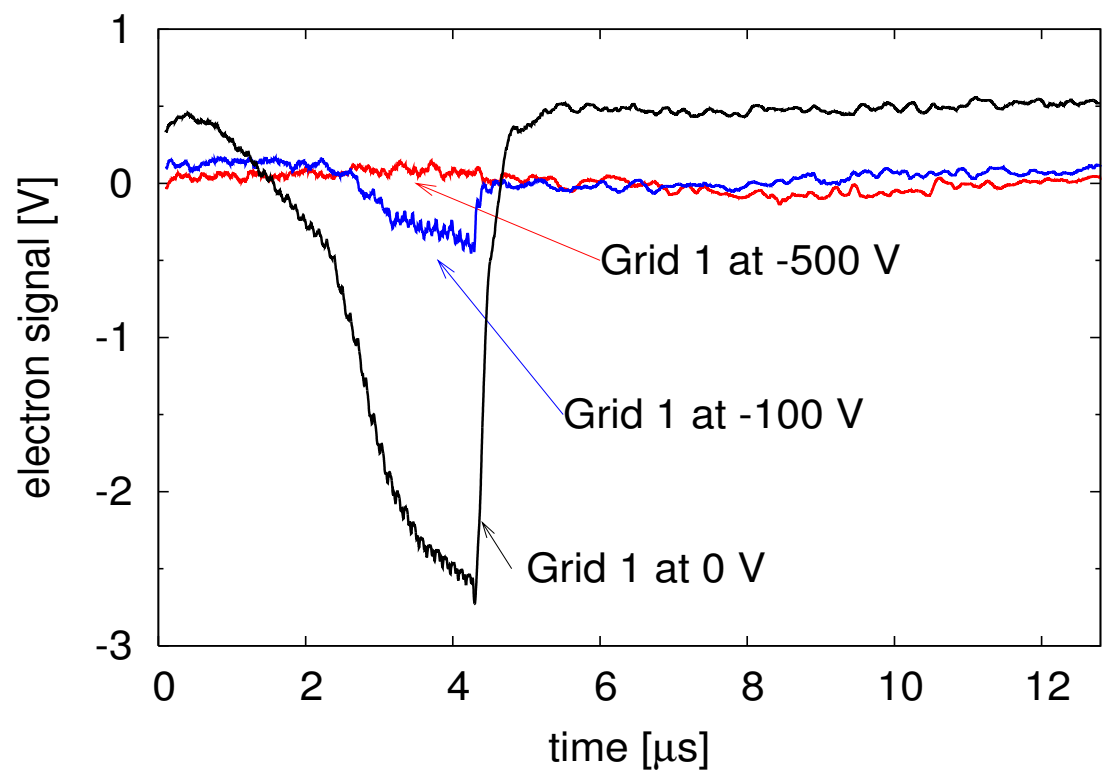

Figure 4.8: Effect of polarizing "grid 1" at different voltages. The electron signal decreases with negative "grid 1" voltages because fewer electrons can reach the collector plate. The signal disappears when the grid voltage is at $-500 \mathrm{~V}$.

2. Figure 4.8 shows three electron signals with different "grid 1" voltages. The three signals have been smoothed with a numerical $10 \mathrm{MHz}$ filter and they correspond to the same fill and the same beam conditions (43 bunches injected $107 \mathrm{~ns}$ apart with an average bunch intensity of $N_{b}=1.6 \times 10^{11}$ protons). When the grid is not polarized ( $V_{\text {grid }}=0 \mathrm{~V}$, black trace), an electron cloud build up is detected. The signal starts with values around $0.5 \mathrm{~V}$, and its minimum value is about $-2.5 \mathrm{~V}$. When the grid is polarized with $V_{\text {grid }}=-100 \mathrm{~V}$ (blue trace), the signal only goes from $\sim 0.1 \mathrm{~V}$ to about $-0.4 \mathrm{~V}$, and for $V_{\text {grid }}=-500 \mathrm{~V}$, the signal disappears. In all cases, the average is null, yet the peak to peak signal significantly decreases by applying a negative voltage to "grid 1". This shows that the signal is due to electron bombardment, and that the signal differentiation is due to the AC coupling of the electron detector.

3. Figure 4.9 shows a Fourier transform of the raw signal in Fig. 4.7. Note the strong components of the multipacting signal at frequencies around the typical ED cutoff frequencies, 30 and $300 \mathrm{kHz}$ - see Appendix A. 
FFT on EC signal

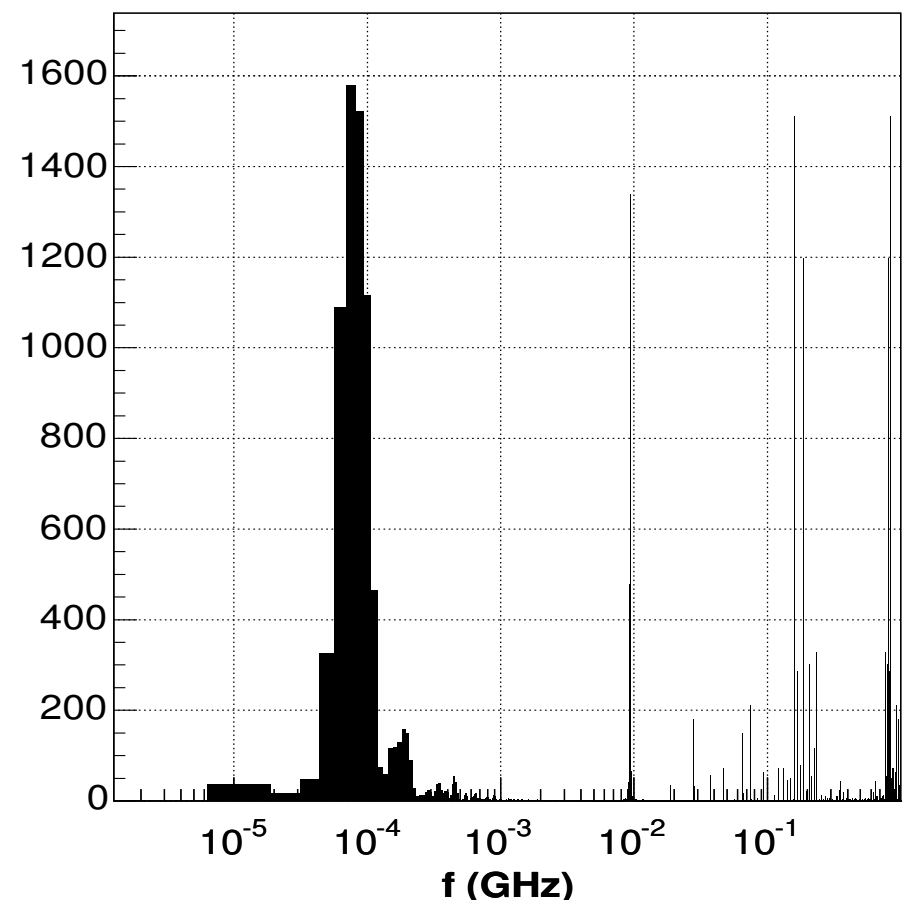

Figure 4.9: Fast Fourier Transform of the raw ED signal (top plot in Fig. 4.7). Strong signal components are comprised between 30 and $300 \mathrm{kHz}$, coinciding with the typical low frequency cut-offs of most of the electron detectors in RHIC.

\subsection{Calibration of the electron detector}

The electron flux into the wall is the quantity one wishes to obtain from the RHIC ED. Two calibrations are required for inferring this quantity from a given ED voltage. One ensures that the signal is not attenuated as a function of the frequency due to the system electronics, cable length, et cetera. The second involves the relation between the voltage and flux into the wall.

\subsubsection{Frequency calibration}

Using a Signal Generator, different sine-wave signals with different frequencies were introduced in the ED system inside the tunnel (at the collector plate, see Fig. 4.2), and measured at the scope inside the service building (see Fig. 4.2). The total cable length is $\approx 150 \mathrm{~m}$. From the peak to peak measurement ratio between the signal generator and the scope value, the frequency calibration shown in Fig. 4.10 is established. This shows that the $3 \mathrm{~dB}$ frequency limit bandwidth is at $50 \mathrm{MHz}$. From this, and in agreement with the PSPICE simulations, the conclusion is that the peak to peak signal at the scope is not affected by the electronics of the system or the cable length. Thus the peak to peak values shown in Figs. 4.7, 4.8 are reliably taken to be the same as the ones at the ED location. 


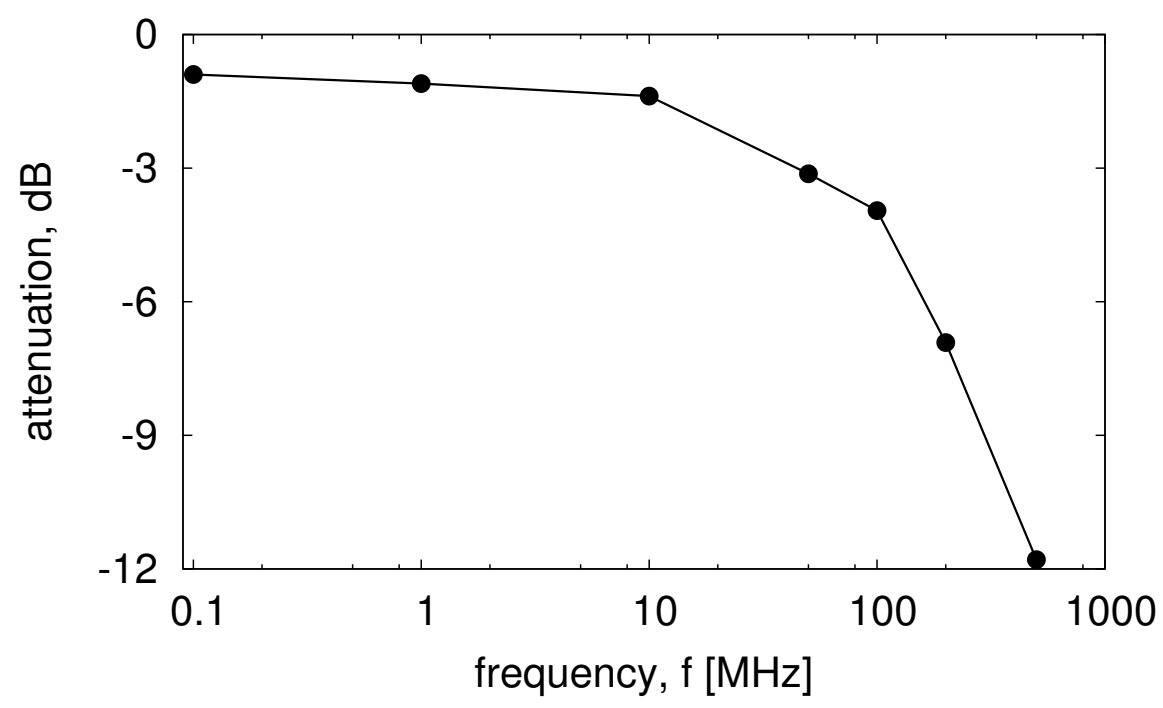

Figure 4.10: Bandwidth calibration of the ED. The measured $3 \mathrm{~dB}$ limit is at about $50 \mathrm{MHz}$ and ensures that signals with lower frequencies are not attenuated by the system electronics.

Alternatively, a frequency calibration for the ED electronics without including the cable length but using a Network Analyzer is shown in Appendix A. The upper frequency limit is only $1 \mathrm{MHz}$, where the attenuation due to the cable length is usually not noticeable.

\subsubsection{Electron flux to the wall}

The electron flux into the wall is used to benchmark the output of simulation codes, and to relate it with the pressure (see Chapters 5 ). For a given signal in the scope, $V$, the flux into the wall per unit area is

$$
\frac{d I}{d A}=\frac{V}{Z G A_{\mathrm{ED}} T_{\mathrm{eff}}}
$$

where $A_{\mathrm{ED}}$ is the surface area of the detector, $Z$ is the line impedance, $G$ is the amplifier gain, and $T_{\text {eff }}$ is the effective transparency of the detector. In principle, assuming no electrons are lost when traversing the path between grid 0 and the collector (see Fig. 4.1) the effective transparency would be estimated as $T_{\text {eff }}=T_{0} T_{1} T_{2} \approx 15 \%$. However this ideal relation does not take into account the electron energy: low energy electrons have a larger probability of being lost between the grids than high energy ones. This effect is calibrated using the test chamber setup shown in Ref. [64]. By means of an electron gun, monoenergetic electron fluxes are sent at different energies to the electron detector. The ratio between the flux at the gun and the flux collected in the electron detector gives the effective transparency. Figure 4.11 shows the results for electron energies $E=5,20,50,100$, and $500 \mathrm{eV}$. By varying the filter grid bias, $V_{\text {filter }}$, one can see that the output is reasonably flat until the filter bias 
reaches approximately the energy of the electron beam, that is, when $\left|e V_{\text {filter }}\right| \approx E$. More details about the calibration process can be found in Refs. [64,65].

Since the energy spectrum of the multipacting electrons at RHIC is expected (and, as seen in Chapter 5, is measured) to have mainly low energy electrons, a good average value for the effective transparency is taken to be $T_{\text {eff }}=5 \% \pm 1 \%$. Therefore, taking into account the typical values of $\mathrm{Z}=50 \Omega, A_{\mathrm{ED}}=78 \mathrm{~cm}^{2}, \mathrm{G}=1600$ (equivalent to $64 \mathrm{~dB}$ ), for a given voltage the corresponding electron flux per unit area is

$$
\frac{d I}{d A}\left[\frac{\mu \mathrm{A}}{\mathrm{cm}^{2}}\right] \approx 3.2 \mathrm{~V}[\mathrm{~V}]
$$

This holds for the signals seen in this chapter (for example, Figs. 4.7, and 4.8).

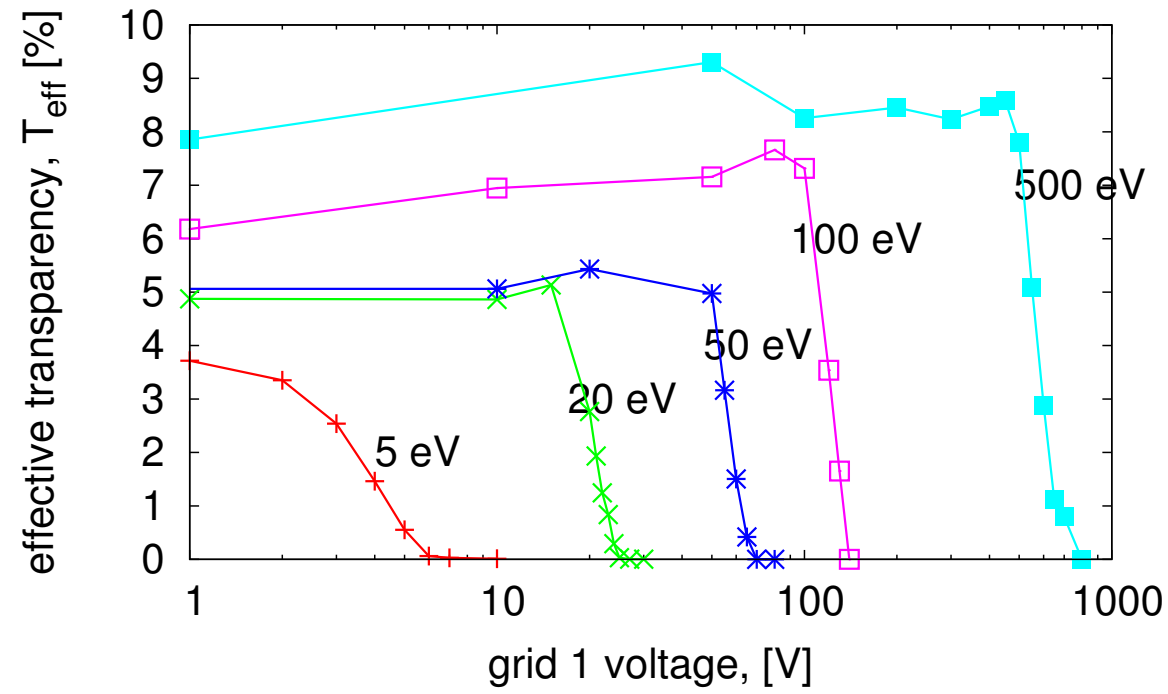

Figure 4.11: Effective transparency of the ED for different electron energies [64]. The grid voltage (horizontal axis) is plotted in absolute value on a logarithmic scale. 



\section{Chapter 5}

\section{Electron induced molecular desorption in the RHIC beam pipes}

The pressure rise dependence on the electron flux to the wall produced after an electron cloud build up is described in this Chapter. The pressure rise and the electron flux are related through the molecular desorption coefficient from electron bombardment. This coefficient is inferred from the analysis of the electron detector and pressure gauge signals, both for baked and unbaked stainless steel. Since the molecular desorption coefficients strongly depend on the energy at which the electron hits the wall, an electron energy spectrum is presented and compared with simulated energy spectra.

\subsection{Pressure rise due to an electron cloud}

Assuming the beam pipe is a periodic structure with vacuum pumps of pumping speed $2 S$ spaced by the distance $2 L$, the pressure distribution along the longitudinal position $z$ between two vacuum pumps is [66]:

$$
P(z)=q\left[\frac{2 L z-z^{2}}{2 c}+\frac{L}{S}\right] \quad, \quad \text { for } 0 \leq \mathrm{z} \leq 2 \mathrm{~L} .
$$

Here the $z$ origin is placed at one of the vacuum pumps, $c$ is the specific molecular conductance of the vacuum chamber, and $q$ is the specific linear outgassing rate. Consider electron cloud situations in which the outgassing rate due to an electron flux $d I / d l$ (in units of $[\mathrm{A} / \mathrm{m}]$ ) exceeds by a wide margin the thermal outgassing rate. In the absence of magnetic fields and assuming a regular and homogeneous chamber, one can further consider the electron flux to be constant throughout the beam pipe. The outgassing rate then does not depend on the longitudinal beam pipe position $z$, and it can be expressed by

$$
q=\eta_{e} \frac{k T}{e} \frac{d I}{d l},
$$

where $e$ is the absolute value of the electron charge, $k$ is Boltzman constant, $T$ is the temperature, and $\eta_{e}$ is the electron induced molecular desorption coefficient of the beam pipe wall, that is, the number of desorbed molecules per impinging electron. 
As seen in Chapter 3, the electron flux $d I / d l$ is not constant in time. Figure 5.1 shows a typical evolution of the electron flux during a RHIC turn in a simulation using CSEC (see Section 3.5). The simulation is for a bunch train of 110 bunches with $8 \times 10^{10}$ protons/bunch followed by a 10 bunch abort gap. The result is numerically smoothed with a $10 \mathrm{MHz}$ filter, which is also used in measurements with the electron detectors. Initially the electron flux grows exponentially, and it saturates after $\approx 8 \mu$ s due to space charge effects [67]. During the abort gap, the electron flux decays rapidly. This behaviour repeats at every turn. Since the time constant of the vacuum pumps is a few seconds [68], the pressure evolution cannot be followed within one turn. The pressure responds then to the time averaged flux over one turn,

$$
\left\langle\frac{d I}{d l}\right\rangle_{\tau}=\frac{1}{\tau} \int_{0}^{\tau} \frac{d I(t)}{d l} d t
$$

where $\tau$ is the revolution period, and $d I(t) / d l$ is the instantaneous electron flux. Using Eqs. 5.1 and 5.2, the pressure due to an electron cloud at a given position $z$ is

$$
P(z)=P_{0}+\eta_{e} \frac{k T}{e}\left\langle\frac{d I}{d l}\right\rangle_{\tau}\left[\frac{2 L z-z^{2}}{2 c}+\frac{L}{S}\right]
$$

where $P_{0}$ is the static pressure.

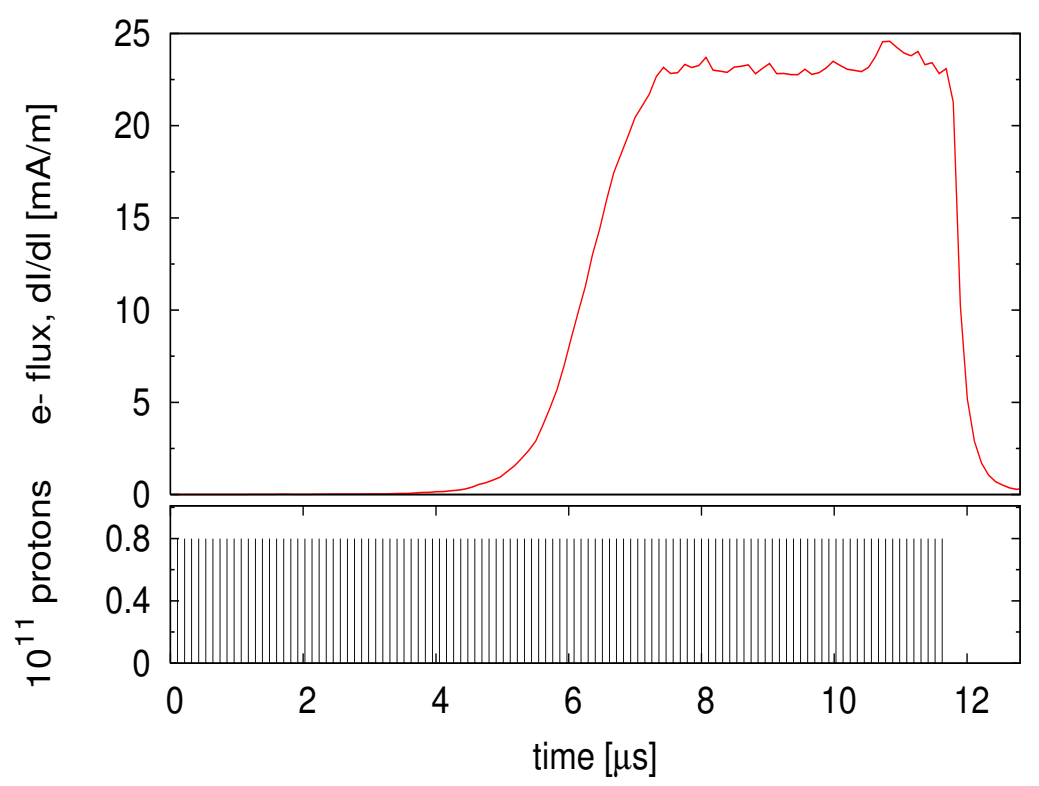

Figure 5.1: The top plot shows a typical evolution of the electron flux during a revolution when RHIC is filled with a train of 110 bunches followed by a 10 bunches abort gap (bottom plot). This is a simulation using CSEC with an intensity of $8 \times 10^{10}$ protons/bunch. The pressure is proportional to the time averaged electron flux in one turn, not to the peak value.

Equation 5.4 shows that, at a given location $z$ the final pressure is directly proportional to the electron desorption coefficient, $\eta_{e}$. This coefficient depends on the energy of the striking 
electron, the surface material, and the accumulated dose on the surface. For electrons below $100 \mathrm{eV}$, only a small amount of data exists in the literature. This is unfortunate, since the energy of the multipacting electrons for RHIC conditions falls within this range. For stainless steel, data for energies as low as $300 \mathrm{eV}$ are found in Ref. [69]. For OFHC Copper and energies as low as $20 \mathrm{eV}$, data can be found in Ref. [70]. The desorption coefficient also changes depending on the released gas. Since the experimental set-up in RHIC does not have a Residual Gas Analyzer to investigate the pressure composition, all calculations are done for $C O$ at room temperature [68]. The pumping speed is obtained from the manufacturer specifications (see Table 5.1).

The following Section tests whether Eq. 5.4 holds for the RHIC case. Then, from the analysis of the experimental data, the desorption coefficient and its time evolution is obtained. This analysis is performed for two different locations, with two different surfaces, and during two different runs. The single beam vacuum chamber at "BO2" during 2003 was unbaked stainless steel. The common beam pipe at "IR12" during 2004 was baked stainless steel.

\subsection{Experimental set up}

\subsubsection{Unbaked surface instrumentation}

During 2003, approximately $60 \%$ of the warm RHIC beam pipes were baked. Beam injection is inhibited when the pressure at any location in the ring reaches an unacceptable level. These limits were first approached within the $40 \%$ of the unbaked regions, where the electron multipacting thresholds are lower than in the baked regions. The pressure rises in the unbaked regions prevented that multipacting conditions in baked regions were reached. In this situation, only the electron detector in an unbaked region (labeled as BO2) is easily exposed to electron clouds, and only a few electron clouds were detected in the baked regions. Therefore, the analysis during 2003 uses the instrumentation in the unbaked single beam pipe shown in Fig. 5.2, which is considered as the periodic structure mentioned in Section 5.1. The vacuum pump and pressure gauge are at the same location, the electron detector is $8 \mathrm{~m}$ away. Since the beam pipe between the ED and the vacuum pump/gauge is made of the same material, one can assume that the electron flux is the same throughout the entire region. In this situation, the pressure at the gauge "pw3.2" $(z=0)$ is related to the electron detector signal using Eq. 5.4,

$$
P(z=0)=P_{0}+\eta_{e} \frac{k T}{e}\left\langle\frac{d I}{d l}\right\rangle_{\tau} \frac{L}{S} .
$$

The same result is found if we place the origin $(z=0)$ at pw3.1 because of the symmetric properties of Eq. 5.1. The flux into the wall $d I / d l$ is rewritten in terms of flux per unit area, $d I / d A$ using the beam pipe radius, $b$, as

$$
\frac{d I}{d l}=\frac{d I}{d A} 2 \pi b,
$$




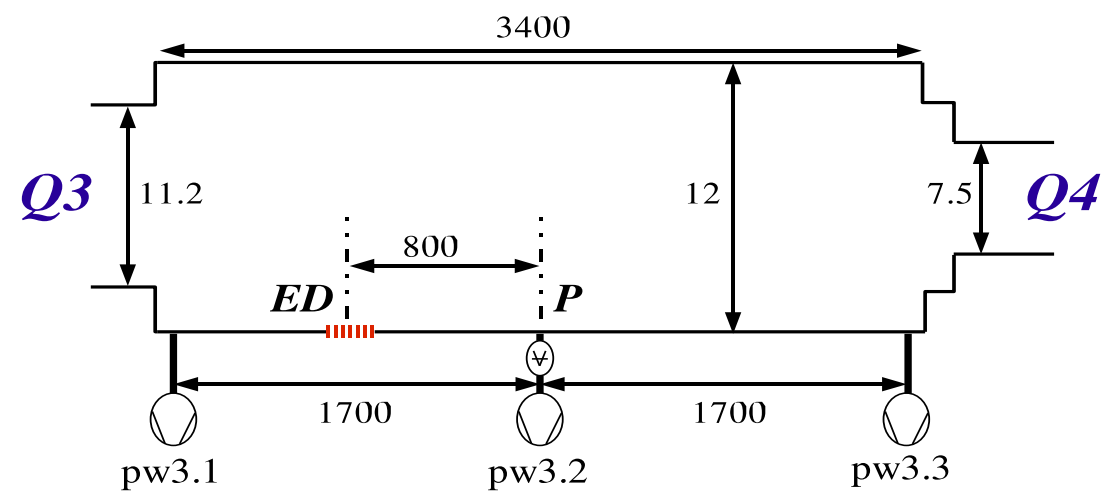

Figure 5.2: Geometry of the single beam pipe BO2, made of stainless steel and unbaked during 2003. The electron detector location (marked with $E D$ ) is about $8 \mathrm{~m}$ away from the pressure gauge and ion pump, whose location is marked with $P$. Distances are given in $\mathrm{cm}$ and are not to scale.

Using Eqs. 4.4 and 5.6, the time averaged over one turn electron flux into the wall $d I / d l$ is related to the time averaged over one turn electron detector voltage $\langle V\rangle_{\tau}$ by

$$
\left\langle\frac{d I}{d l}\right\rangle_{\tau}=\frac{2 \pi b}{Z G A_{\mathrm{ED}} T_{\mathrm{eff}}}\langle V\rangle_{\tau}
$$

where, as in Chapter $4, A_{\mathrm{ED}}$ is the area of the electron detector at the beam pipe wall, $Z$ is the line impedance, $G$ is the amplifier gain, and $T_{\text {eff }}$ is the effective transparency of the electron detector. $T_{\text {eff }}$ depends on the electron energy, and a good average value is $5 \%$ (see Fig. 4.11). For a given voltage, and taking into account Eq. 4.5 and the parameters in Table 5.1, the corresponding electron flux per unit area is

$$
\frac{d I}{d A}\left[\frac{\mu \mathrm{A}}{\mathrm{cm}^{2}}\right] \approx 3.2 V[\mathrm{~V}]
$$

The electron flux is obtained from the RHIC electron detector described in Chapter 4. Typically, the ED takes a snapshot of the electron signal during one turn every 4 seconds. The signal has a time resolution of $1 \mathrm{~ns}$, and it is smoothed with a $10 \mathrm{MHz}$ filter. As seen in Chapter 4, the electron detector is $\mathrm{AC}$ coupled to the system electronics. To calculate a nonzero average over one turn the baseline is shifted by the maximum value in the electron detector snapshot. The average over one revolution is then calculated by

$$
\langle V\rangle_{\tau}=\frac{1}{\tau} \int_{0}^{\tau} V(t) d t=\frac{1}{N} \sum_{i=1}^{N}\left[V_{i}-V_{\max }\right],
$$

where $N$ is the number of samples in one revolution. Using Eqs. 5.5 and 5.7, the pressure as a function of the electron detector voltage is expressed by

$$
P=P_{0}+\eta_{e} \frac{k T}{e} \frac{L}{S} \frac{2 \pi b}{Z G A_{\mathrm{ED}} T_{\mathrm{eff}}}\langle V\rangle_{\tau} .
$$




\subsubsection{Baked surface instrumentation}

Approximately $80 \%$ of the warm beam pipes in RHIC were baked by 2004, including the aforementioned section BO2. During the polarized proton run, electron clouds were often detected in the common beam pipe, named as "IR12". As will be seen in Chapter 7, two beams in opposite directions traversing a common beam pipe produce either larger bunch intensities than in the single beam pipes, or shorter bunch spacings than the ones in the single beam pipes. Therefore, electron cloud thresholds in the common beam pipe regions are more easily created than in the single beam pipes. Data is taken using the instrumentation in the common (and baked) beam pipe shown in Fig. 5.3. The electron detector, vacuum pump, and pressure gauges are only $0.3 \mathrm{~m}$ apart. All this instrumentation is assumed to be at the same location, and use Eq. 5.4 for $z=0$. Thus, the calculation of the pressure as a function of the voltage in the ED again follows Eq. 5.10 using the values for baked surfaces in Table 5.1.

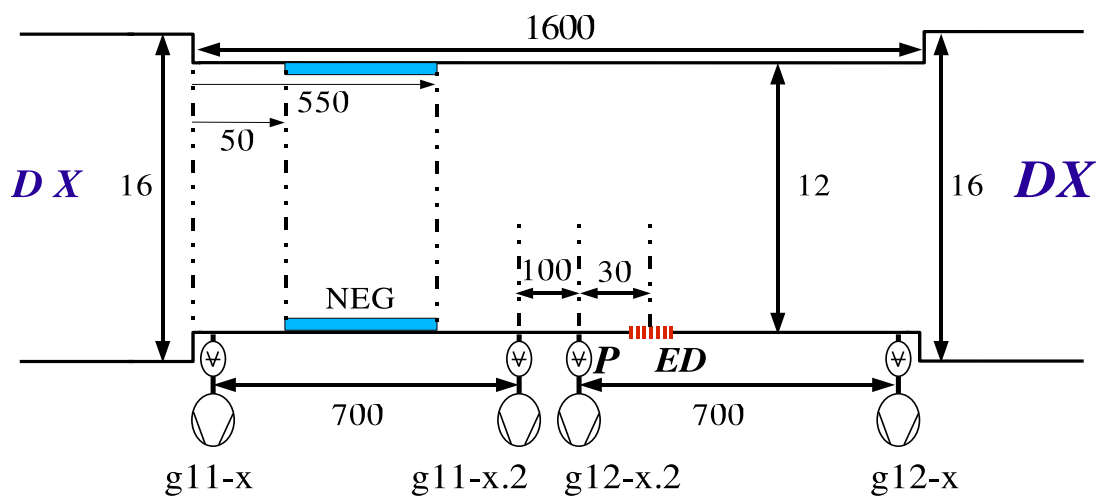

Figure 5.3: Geometry of the common beam pipe IR12, made of baked stainless steel. The electron detector location (marked $E D$ ) is only $0.3 \mathrm{~m}$ away from the pressure gauge and vacuum ion pump, whose location is marked $P$. Distances are given in $\mathrm{cm}$ and are not to scale.

Table 5.1: Parameters used to estimate the electron desorption coefficient for the baked and unbaked surface.

\begin{tabular}{|l|c|c|cc|}
\hline \hline parameter & symbol & unit & $\begin{array}{c}\text { value unbaked } \\
\text { location }\end{array}$ & $\begin{array}{c}\text { value baked } \\
\text { location }\end{array}$ \\
\hline distance between pumps & $2 L$ & $\mathrm{~m}$ & 17 & 7 \\
pumping speed for $C O$ & $2 S$ & $\mathrm{l} / \mathrm{s}$ & 140 & 270 \\
beam pipe radius & $b$ & $\mathrm{~cm}$ & \multicolumn{2}{|c|}{6} \\
impedance line & $Z$ & $\Omega$ & \multicolumn{2}{|c|}{50} \\
amplifier gain & $G$ & - & \multicolumn{2}{|c|}{1600} \\
electron detector area & $A_{\mathrm{ED}}$ & $\mathrm{cm}^{2}$ & \multicolumn{2}{|c|}{78} \\
effective transparency & $T_{\mathrm{eff}}$ & $\%$ & \multicolumn{2}{|c|}{5} \\
\hline \hline
\end{tabular}




\subsection{Electron induced desorption of unbaked stainless steel}

Figure 5.4 shows an example of experimental data. The top plot shows the time evolution of the pressure at the gauge pw3.2 (red line, right vertical axis), and the electron signal averaged over one turn (black dots, left vertical axis, calculated using Eq. 5.9) as beam is injected (bottom plot). At about $16 \mathrm{~h} 17 \mathrm{~m} 30 \mathrm{~s}$, the injection is interrupted and the beam is dumped due to the large pressure in the RF cavities.
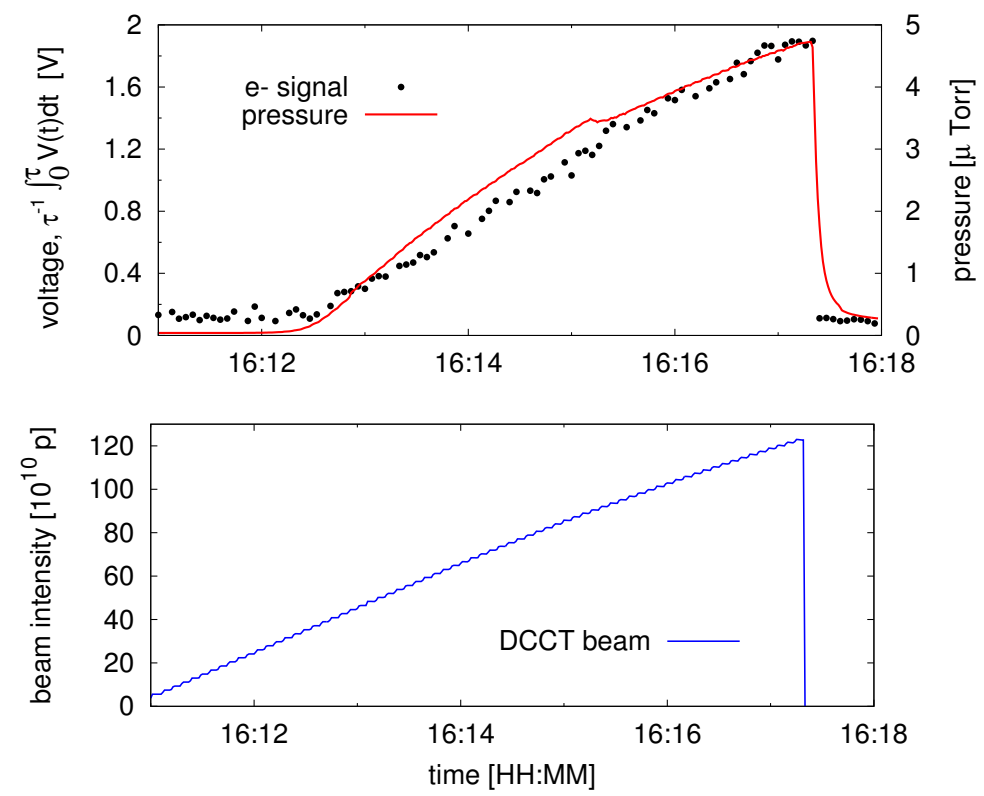

Figure 5.4: Pressure and eletron signal evolution (top plot), as the blue beam is being injected (bottom plot). Pressure and electron signal follow a similar evolution.

A linear relation between the pressure readings and the electron signal averaged over one turn is confirmed in Fig. 5.5, which validates the initial assumption in Eq. 5.4, and supports the idea of electron clouds as the driving mechanism for the pressure rises in RHIC. This linear relation has been also found in other accelerators $[71,72]$. The black line in Fig. 5.5 shows the result of a linear regression applied to the red points,

$$
P=A+B\langle V\rangle_{\tau}
$$

where $A$ and $B$ are the fitting coefficients. The independent term $A$ is given by the static pressure and the electron detector signal baseline. It follows that the desorption coefficient, $\eta_{e}$ is derived from the fit coefficient $B$ through

$$
\eta_{e}=B \frac{e}{k T} \frac{S}{L} \frac{Z G A_{\mathrm{ED}} T_{\mathrm{eff}}}{2 \pi b}
$$




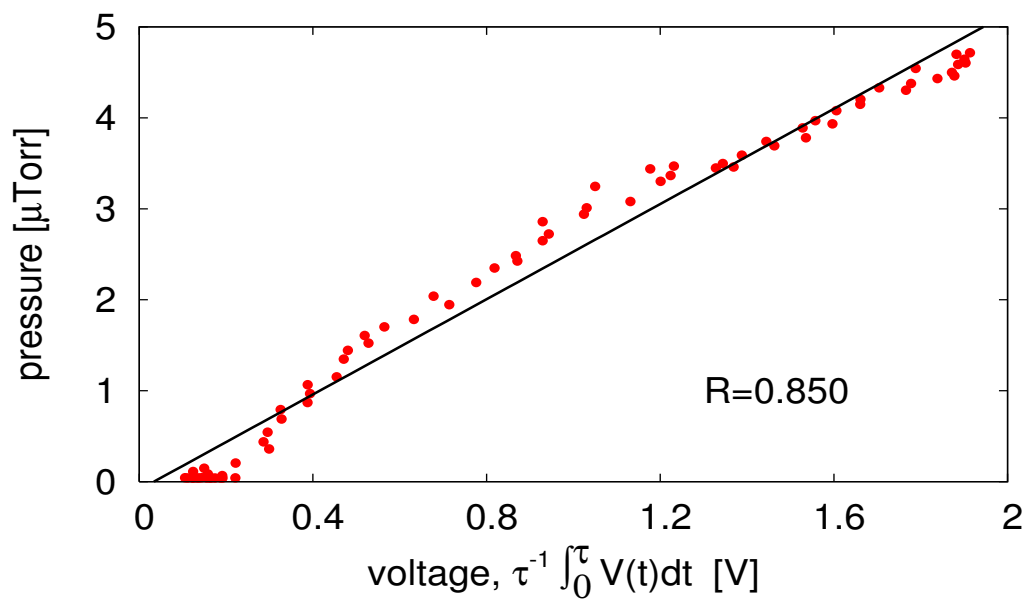

Figure 5.5: Linear fit (black line) to the experimental data (red points) from Fig. 5.4. The fit includes all the data until the beam is dumped. The linear relation between the pressure and the ED voltage $\langle V\rangle_{\tau}$ is confirmed, in this case, for an unbaked stainless steel surface.

For the case in Fig. 5.4, the correlation coefficient is $R=0.850$, the error in $B$ is $2 \%$, and the desorption coefficient $(C O$ equivalent $)$ is $\eta_{e}=0.01 \pm 0.005 \%$ molecules/electron. The error in $\eta_{e}$ stems from the uncertainty in the pressure reading and pumping speed values [68]. The injection shown in Figs. 5.4, and 5.5 took place at the end of the run, after the surface was conditioned by electron bombardment for several weeks during operation.

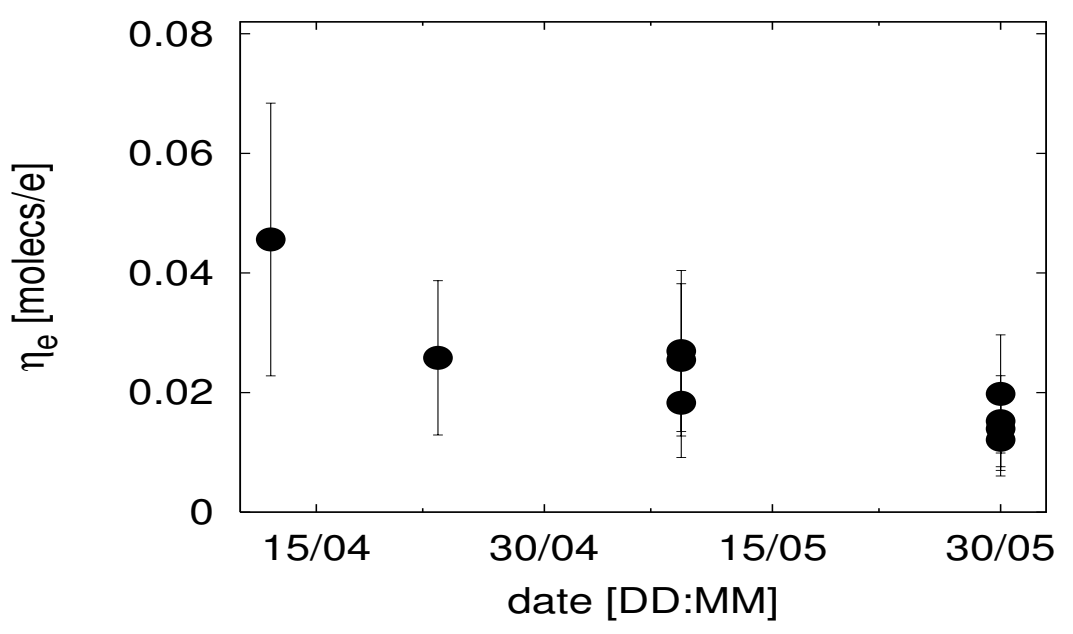

Figure 5.6: Summary of all calculated desorption coefficients for the unbaked surface BO2. The error bar (50\%) stems from the uncertainty in the pumping speed and vacuum pressure readings. A slight decrease of the desorption coefficient with time is noticeable due to the scrubbing effect. 
This measurement of the desorption coefficient was performed for all the fills during 2003 that produced electron detector signals above the noise level. Figure 5.6 shows the evolution of the calculated desorption coefficient until the end of the run. As expected, this coefficient decreases with time due to the bombardment dose. In about 6 weeks, $\eta_{e}$ decreased by almost a factor of 5. An estimate of the total bombardment dose is difficult. The signal-to-noise ratio of the electron detector does not allow electron signals to be obtained under about $0.15 \mathrm{~V}$, even though pressure rises are observed.

\subsection{Electron induced desorption of baked stainless steel}

An example of an electron cloud, and its correlation with the pressure in IR12 is shown in Fig. 5.7. Once injection into the blue ring has finished (blue line, bottom plot), the electron cloud is triggered after approximately 36 bunches are injected in the yellow ring (red line, bottom plot), and both the pressure (red trace, top plot) and the electron signal (black points, top plot) start increasing at the same rate. Note the "swing" in both the electron signal and the pressure evolution (top plot), as the bunch length (denoted with blue and red squares for the blue and yellow beam, respectively) swings during the ramp (bottom plot). Shorter bunches produce electrons with larger striking energies at the wall, which translates into a larger secondary emission yield and a larger flux into the wall.
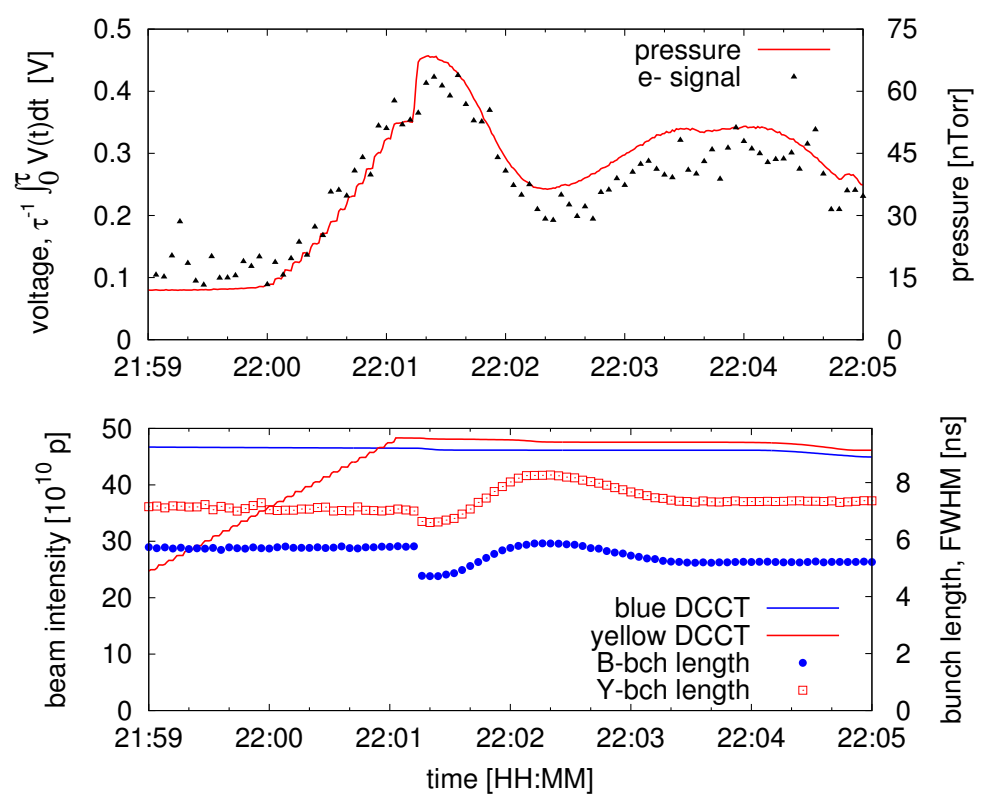

Figure 5.7: Dynamic pressure evolution and electron signal (top plot), as the blue beam is being injected (bottom plot) for fill 5201. At the beginning of the energy ramp (22:01:20) the rf voltage is raised leading to shorter bunches. 
Again, the correlation between pressure and electron detection is confirmed when plotting the electron detector voltage versus the pressure (see Fig. 5.8). For the particular case in Fig. 5.8, the calculated desorption coefficient is $\eta_{e}=0.004 \pm 0.002 \%, \mathrm{R}=0.942$, and the error in $\mathrm{B}$ is $3.7 \%$.

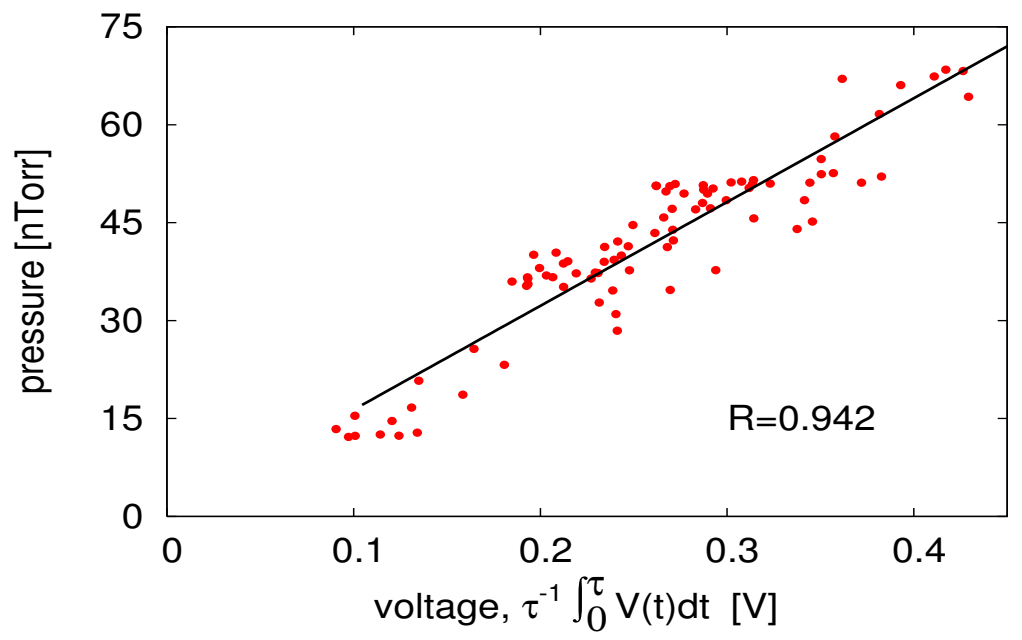

Figure 5.8: Linear fit (black line) to the experimental points (red dots) shown in Fig. 5.5. As for the unbaked surface, a linear relation between the electron signal and the pressure is confirmed.

Figure 5.9 shows the analysis for all the fills that produced electron signals in IR12 during 2004. Note that no scrubbing effect is seen. It is possible that the chamber is sufficiently conditioned by baking, or that the low energy electrons do not lead to observable conditioning. This could also be due to the presence of the hydrogen jet installed to measure beam polarization. The jet injects an extra gas load, and runs almost continously to measure the beam polarization [73]. If one understands the scrubbing as a "cleaning" effect, the injection of gas can cancel this effect. The average and standard deviation of the measurement shown in Fig. 5.9 are $\eta_{e}=0.004 \pm 0.001$ molecules/electron. Compared with the unbaked surface, this is about one order of magnitude larger before the scrubbing performed during the six weeks of machine operation.

A direct comparison between the desorption coefficient obtained from this analysis and from the literature is difficult because it involves different surfaces, different electron energy ranges, bombardment doses at different energies, et cetera. Reference [69] reports on the variation of the stainless steel desorption coefficient with the temperature, fixing the incident electron energy at $300 \mathrm{eV}$. A fiducial value for $C O$ is $\eta_{e}=0.01$ molecules/electron. For the "as received" OFHC Copper reported in Ref. [70], the $C O$ desorption coefficient decreases from $\eta_{e}=0.05$ molecules/electron for $300 \mathrm{eV}$ electron energies to $\eta_{e}=0.005$ molecules/electron at $20 \mathrm{eV}$, and Ref. [74] shows that this value can decrease by 3 orders of magnitude after a proper bombardment dose. All in all, the conclusion is that results for both baked and unbaked surfaces agree in their order of magnitude with laboratory measurements $[69,70,74]$. 


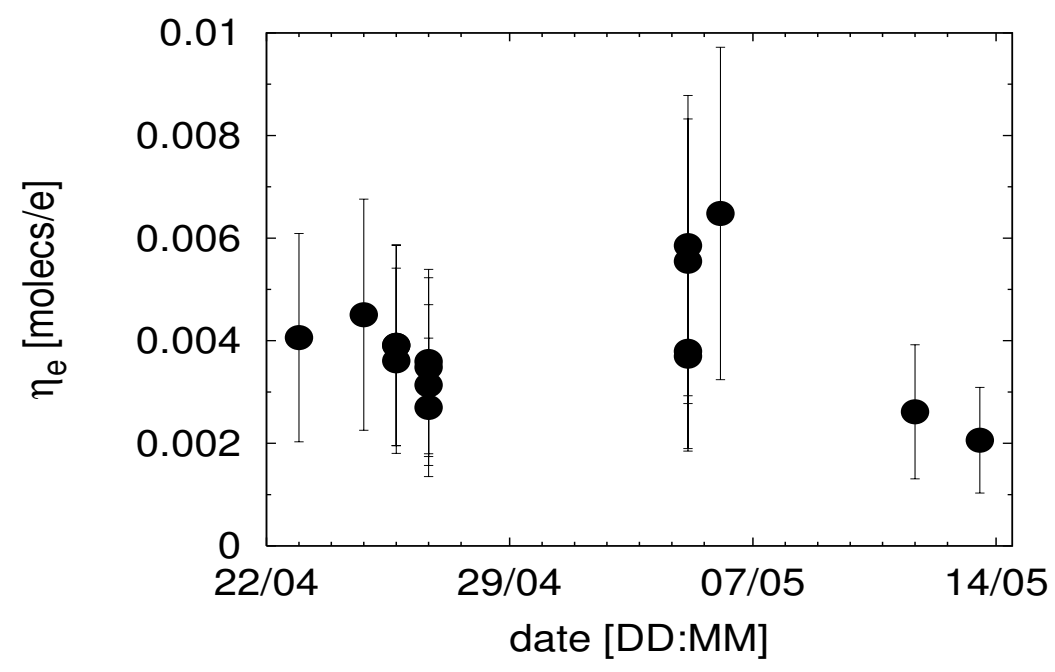

Figure 5.9: Summary of all calculated desorption coefficients for the baked staineless steel surface at IR12 during 2004. The error bar is about $50 \%$ of the calculated value due to uncertainty in the pumping speed and vacuum pressure readings. The average and rms are $0.004 \pm 0.001$ molecules/electron.

\subsection{Electron energy during multipacting conditions}

\subsubsection{Experimental results using the electron detector}

The desorption coefficient strongly depends on the energy of the electron when it strikes the beam-pipe wall. The energy spectrum of the cloud electrons was measured during a fill for the unbaked surface (see Fig. 5.2). The measurement was taken during a beam experiment with bunch intensities ranging from $N_{b}=1.4$ to almost $2 \times 10^{11}$ protons, about twice as large as the operational bunch intensities at the time.

Several energy sweeps were carried out using the instrumentation in Fig. 4.1. These sweeps consist in ramping "grid 1" from 0 to $-500 \mathrm{~V}$ (with $-500 \mathrm{~V}$, no electron flux is detected). The peak ED signal is proportional to the number of electrons whose energy is larger than $\left|e V_{\text {grid }}\right|$. In this way an integrated spectrum is obtained. The right hand side plot in Fig. 5.10 shows the absolute peak electron signal as a function of "grid 1 " voltage $\left(\left|V_{\text {grid }}\right|\right)$ for two different sweeps (curves with red dots and black triangles). As expected, an increase in the grid voltage reduces the peak electron signal. The signal rapidly decreases between 0 and almost $100 \mathrm{~V}$, and it is close to the noise level when the grid voltage (in absolute value) is larger than $300 \mathrm{~V}$.

The derivatives of each curves provide the flux at the particular energy $\left|e V_{\text {grid }}\right|$. The normalized electron flux at a given energy (or grid voltage) is calculated using the central differences method, where the bins with index $i \pm 2$ are used (instead of the usual $i \pm 1$ ) to 
minimize noise oscillations:

$$
\frac{1}{N_{e}} \frac{d N_{e}\left(E_{i}\right)}{d E}=\frac{1}{V_{0}} \frac{V_{\mathrm{i}+2}-V_{\mathrm{i}-2}}{E_{\mathrm{i}+2}-E_{\mathrm{i}-2}},
$$

where $E_{i}=\left|e V_{\text {grid }}\right|$, and $V_{0}$ is the electron signal for a null "grid 1" bias voltage, $V_{\text {grid }}=0 \mathrm{~V}$. In this case, the peak electron signals are obtained through the MADC card (see Section 4.3.1) because its sampling rate $(1 \mathrm{~Hz})$ is faster than the scope mode $(0.25 \mathrm{~Hz})$, and because the MADC is synchronized to the power supply in "grid 1". Attenuations in the MADC processing are not important because Eq. 5.13 only accounts for relative values.

The right hand side plot in Fig. 5.10 shows the normalized electron flux calculated from the integrated spectra (left hand side plot) using Eq. 5.13. The two main features are the large peak of low energy electrons $(\approx 10 \mathrm{eV})$, and a spectrum extending to about $300 \mathrm{eV}$. Since the noise in the ED is around $0.15 \mathrm{~V}$, the upper energy limit for the energy spectrum cannot be concluded definitely.
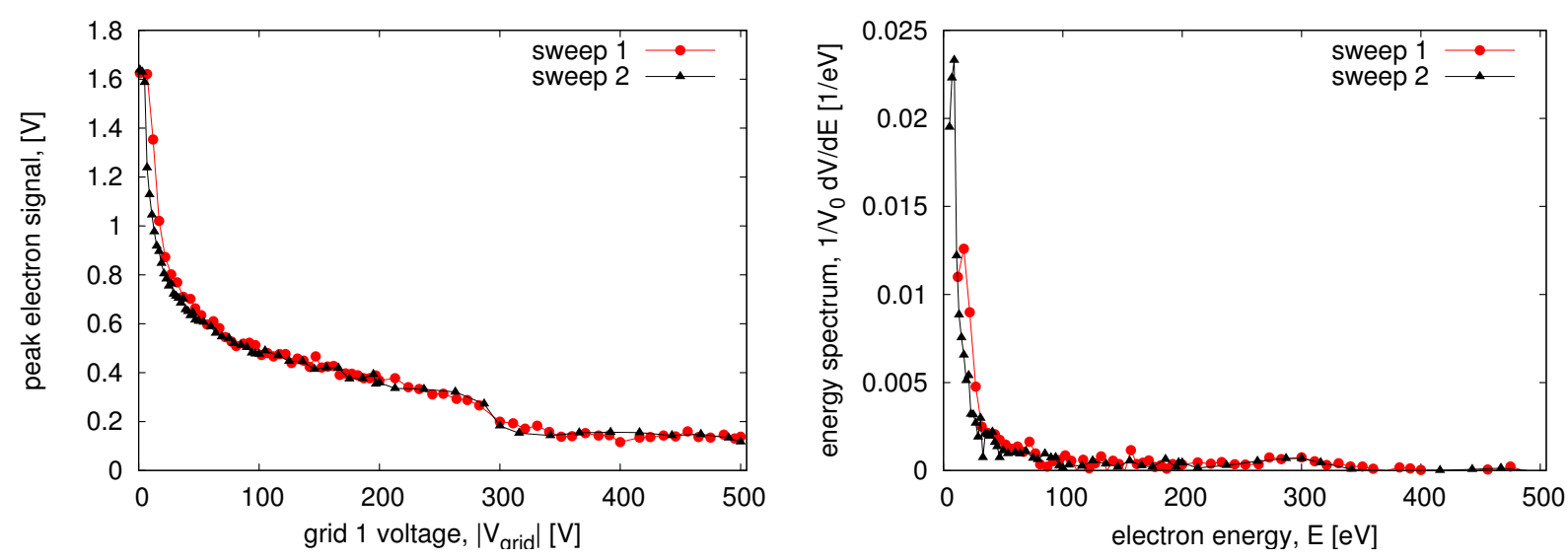

Figure 5.10: Two measured integrated energy spectrum (left hand side plot), and the derived electron distribution (right hand side plot) for energies between 0 and $500 \mathrm{eV}$. The amplitude of the noise oscillations is about $0.15 \mathrm{~V}$.

\subsubsection{Simulation results using CSEC}

Table 5.2 lists the main beam parameters with which the energy spectrum was taken. The electron cloud behaviour has been reproduced using the code CSEC (see Section 3.5) to compare the electron energy spectrum obtained in measurement and simulation. A key ingredient for all simulations is the Secondary Electron Yield (SEY) as a function of the primary energy. In this case, CSEC follows the parameterization and data found in Ref. [46], whose main parameters are listed in the second part of Table 5.2. These parameters have not been measured in situ, thus their values are unknown. For illustrative purposes, the same case is simulated with two different values of the maximum SEY: $\delta_{\max }=1.65$ (left plot in Fig. 5.11), and $\delta_{\max }=2.05$ (right plot in Fig. 5.11). In both cases, a scan in the bunch intensities is performed from $N_{b}=1.4$ to $2 \times 10^{11}$ protons, in steps of $0.2 \times 10^{11}$ protons. For an easy comparison, the plot include the two experimental results from Fig. 5.10. 
It is remarkable that, for all tested bunch intensities $N_{b}$, the energy spectrum shape is approximately the same for both $\delta_{\max }=1.65$ or $\delta_{\max }=2.05$ (specially for energies larger than $\approx 100 \mathrm{eV}$ ). This is an indication that the energy spectrum in RHIC mainly depends on the beam parameters. The simulated energy spectrum is not far off from the experimental data, although the agreement could be better. The comparison between experimental and simulated electron energy spectra is summarized as follows:

- Both the experimental and the simulated results show a large peak of low energy electrons. While the experimental spectrum peaks at about $10 \mathrm{eV}$, the simulated spectrum peaks at $0 \mathrm{eV}$. For low energies the transparency decreases (see Fig. 4.11). Thus, although electrons below $\approx 5 \mathrm{eV}$ hit the inner part of the chamber surface, their experimental detection is difficult.

- For bunch intensities between 1.6 and $2.0 \times 10^{11}$ protons, the spectra in Fig. 5.11 extends to about $300 \mathrm{eV}$, as do the experimental spectra. The reliability of the experimental results for high energy electrons (about $\approx 300 \mathrm{eV}$ ) is low because the flux signal at these energies is similar to the noise level.

Table 5.2: List of beam parameters during the energy spectrum measurement. The second part of the Table shows the SEY parameters used in the CSEC simulations, described in Ref. $[8,46]$.

\begin{tabular}{|l|c|c|c|}
\hline \hline parameter & symbol & unit & value \\
\hline number of bunches & $\mathrm{n}$ & $\ldots$ & 60 \\
average bunch population & $N_{b}$ & protons & $1.6 \times 10^{11}$ \\
bunch spacing & $s_{b}$ & $\mathrm{~ns}$ & 107 \\
full bunch length & $2 \sigma_{z}$ & $\mathrm{~ns}$ & 15 \\
rms bunch radius & $\sigma_{r}$ & $\mathrm{~mm}$ & 2.0 \\
chamber radius & $b$ & $\mathrm{~mm}$ & 60 \\
revolution time & $\tau$ & $\mu s$ & 12.8 \\
\hline maximum SEY & $\delta_{\max }$ & $\ldots$ & 1.65, and 2.05 \\
reflection probability for $E \rightarrow 0$ & $\delta_{0}$ & $\ldots$ & 0.6 \\
reflection probability for $E \rightarrow \infty$ & $\delta_{\infty}$ & $\ldots$ & 0.15 \\
energy at maximum SEY & $E_{\max }$ & $\mathrm{eV}$ & 305 \\
reflection energy & $E_{r}$ & $\mathrm{eV}$ & 60 \\
energy of secondary emitted electrons & $E_{\mathrm{sec}}$ & $\mathrm{eV}$ & 8.9 \\
distribution width of secondary electrons & $\sigma_{\mathrm{sec}}$ & $\mathrm{eV}$ & 5 \\
SEY exponent & $s$ & $\ldots$ & 1.83 \\
\hline \hline
\end{tabular}

No electron energy spectrum could be taken using the instrumentation in the baked surface due to technical difficulties with the power supply of the grid voltage. Nonetheless, the energy spectrum measured and simulated in Fig. 5.11 gives a hint for the low RHIC surface conditionning: energetic electrons are more efficient at scrubbing the surface than low energy electrons. This statement is valid for both for the electron desorption coefficient $\eta_{e}$, and for they SEY [70,75]. Usually, for the same beam conditions the electron fluxes in 
baked surfaces are smaller, and thus, unobservable conditioning of its surface (as seen in Fig. 5.9) is not surprising.
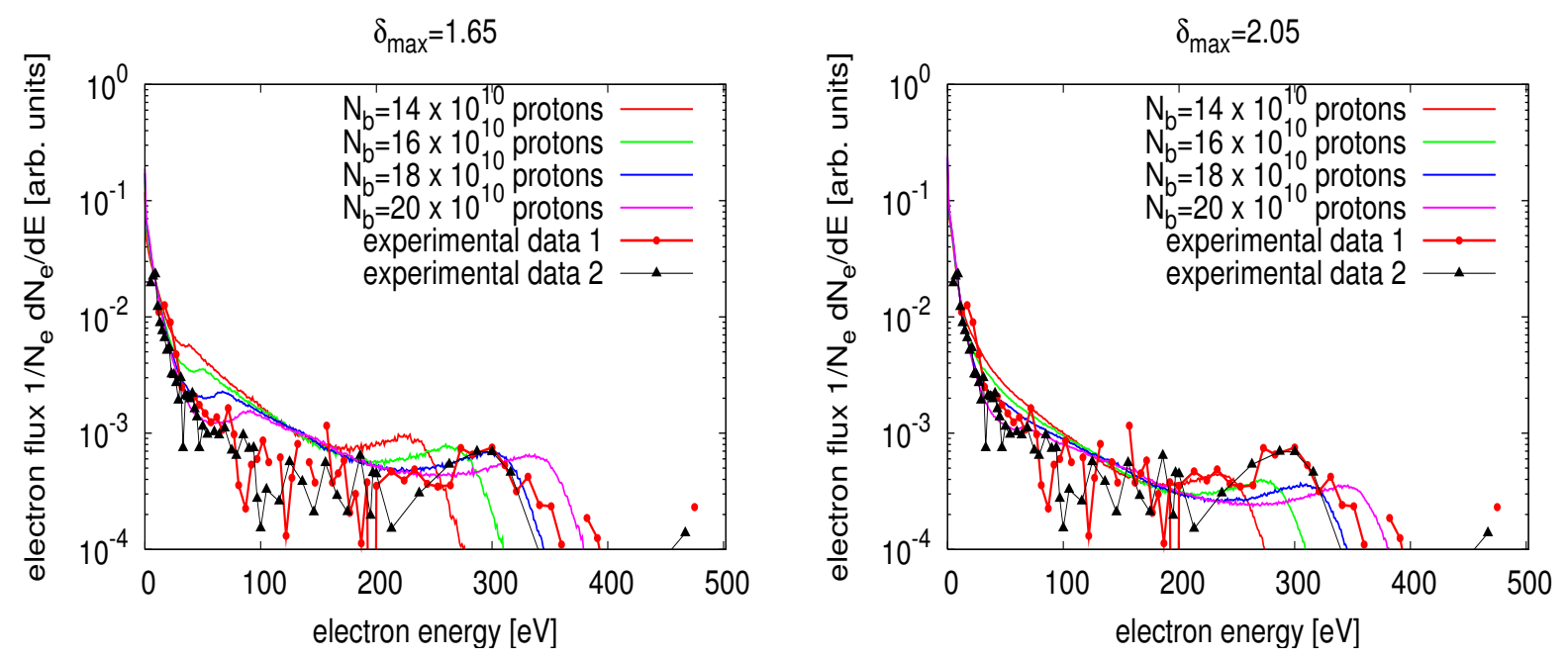

Figure 5.11: Energy spectrum for $\delta_{\max }=1.65$ (left) $\delta_{\max }=2.05$ (right) and different bunch intensities. The spectrum shape is not significantly affected by the surface status, but it depends on the beam parameters (bunch intensity, length, etc).

\subsection{Summary}

A linear relation between the pressure and the electron flux into the wall due to an electron cloud has been observed. The electron desorption coefficient $\eta_{e}$ is inferred from the analysis of the experimental data. For unbaked stainless steel and assuming $C O$ equivalent presure, this value is about 0.05 molecules/electron at the beginning of the run, and decreases to 0.01 molecules/electron after 6 weeks of machine operation due to scrubbing. For baked stainless steel, this value is around 0.005 molecules/electron, and no scrubbing effect is noticeable. For unbaked stainless steel, a measure of the energy spectrum shows a large peak around $10 \mathrm{eV}$. The spectrum extends to at least $300 \mathrm{eV}$. This is in a general good agreement with the spectrum obtained using CSEC simulations. 



\section{Chapter 6}

\section{Electron clouds during beam injection}

This chapter is devoted to the machine limitations produced by electron clouds while the beam is injected into the RHIC ring. These limitations consist of large pressure rises in the warm sections, and have been observed with all species ( $\mathrm{Au}, \mathrm{d}, \mathrm{p}$, and $\mathrm{Cu}$ ). In the cold regions, pressure rises (or gas density increases) occurred only in few instances and have not (yet) limited the machine operation. Simulations show beam instabilities require electron densities about two orders of magnitude larger than the ones usually produced in RHIC [28]. Beam instabilities driven by electron clouds were observed only in Run 5 with unusually large $\mathrm{Cu}$ bunch intensities when the beams crossed the transition energy [76]

Bunches are injected into RHIC one by one, or four at a time, up to a maximum of 110 [7]. This allows to study the evolution of the electron flux and pressure as the beam bunches are being injected. Injection of 110 bunches lasts at least 30 seconds. The user can halt the injection of the next bunch(es) at any time for as long as it is necessary. This Chapter presents the pressure evolution as the beam bunches are being injected, and benchmarks the electron detector snapshots against simulation results.

\subsection{Introduction}

Chapter 5 showed that the pressure, $P$, due to an electron cloud is proportional to the time averaged electron flux to the wall, $\langle d I / d l\rangle$. It is convenient to use the average bunch-to-bunch electron flux expressed in terms of the bunch number $m$,

$$
\phi(m)=\frac{1}{s_{b}} \int_{m}^{(m+1) s_{b}} \frac{d I(t)}{d l} d t
$$

where $s_{b}$ is the time between bunches. If $M$ is the total number of possible bunches, then

$$
\langle\phi\rangle_{M}=\frac{1}{M} \int_{0}^{M} \phi(m) d m=\frac{1}{\tau} \int_{0}^{\tau} \frac{d I(t)}{d l} d t=\left\langle\frac{d I(t)}{d l}\right\rangle_{\tau} .
$$

Using Eq. 5.5 and 6.2, the evolution of the pressure as a function of the injected bunches is

$$
P=P_{0}+\eta_{e} \frac{L k T}{e S}\left[\frac{1}{M} \int_{0}^{M} \phi(m) d m\right],
$$


where $P_{0}$ is the pressure in static conditions, $2 S$ is the pumping speed of the vacuum pump, $2 L$ is the distance between pumps, $k$ is Boltzmann constant, $T$ corresponds to the temperature, and $\eta_{e}$ is the electron desorption coefficient. Note that only the time dependence is considered (through the bunch passage). The spatial distribution along the beam pipe can be inferred using Eq. 5.4. For simplicity and following the assumptions in Chapter $5^{*}$, it has been assumed that $z=0$.

\subsection{Electron flux and pressure evolution during beam injection}

To illustrate the relevant issues of an electron cloud build up as a function of the bunch passage $m$, a typical evolution of the bunch-to-bunch electron flux to the wall during one turn is shown in Fig. 6.1. The maximum number of bunches that can be injected into RHIC (not counting the abort gap) is $\mathrm{M}=120$. The red circles correspond to a simulation result using CSEC (see Chapter 3.5) for a bunch train of 110 bunches with $10^{11}$ protons/bunch. The observed initial exponential growth going to a saturated value is well fitted (black line in Fig. 6.1) using [77]

$$
\phi(m)=\phi_{s} \frac{e^{\left(m-m_{0}\right) / B}}{1+e^{\left(m-m_{0}\right) / B}},
$$

where $\phi_{s}$ represents the electron saturated flux, $m_{0}$ is the bunch corresponding to a flux $\phi_{s} / 2$, and $B$ controls the rise time. These parameters depend on the beam and wall surface parameters.

Following Eqs. 6.3 and 6.4, the pressure as the beam bunches are being injected into the ring is

$$
P(m)=P_{0}+A_{P} \ln \left[1+e^{\left(m-m_{0}\right) / B}\right]
$$

where the constant $A_{P}$ has been introduced:

$$
A_{P}=\eta_{e} \frac{L k T}{e S} \frac{\phi_{s}}{M B} .
$$

Note that both the electron flux, Eq. 6.4, (and consequently, the pressure, Eq. 6.5) show two different regimes:

1. For $m \ll m_{0}$ (and $m_{0} / B \gg 1$, as it is seen a posteriori) the factor $e^{\left(m-m_{0}\right) / B} \ll 1$, and the electron flux is

$$
\phi(m)=\phi_{s} \frac{e^{\left(m-m_{0}\right) / B}}{1+e^{\left(m-m_{0}\right) / B}} \approx \phi_{s} e^{\left(m-m_{0}\right) / B},
$$

while the pressure becomes

$$
P(m)=P_{0}+A_{P} \ln \left(1+e^{\left(m-m_{0}\right) / B}\right) \approx P_{0}+A_{P} e^{\left(m-m_{0}\right) / B} .
$$

Both the electron flux and the pressure exhibit an (initial) exponential growth.

${ }^{*}$ The beam pipe is considered as a periodic structure of vacuum pumps of pumping speed $2 S$ spaced by a distance $2 L$, with $z=0$ at the pump locations. 


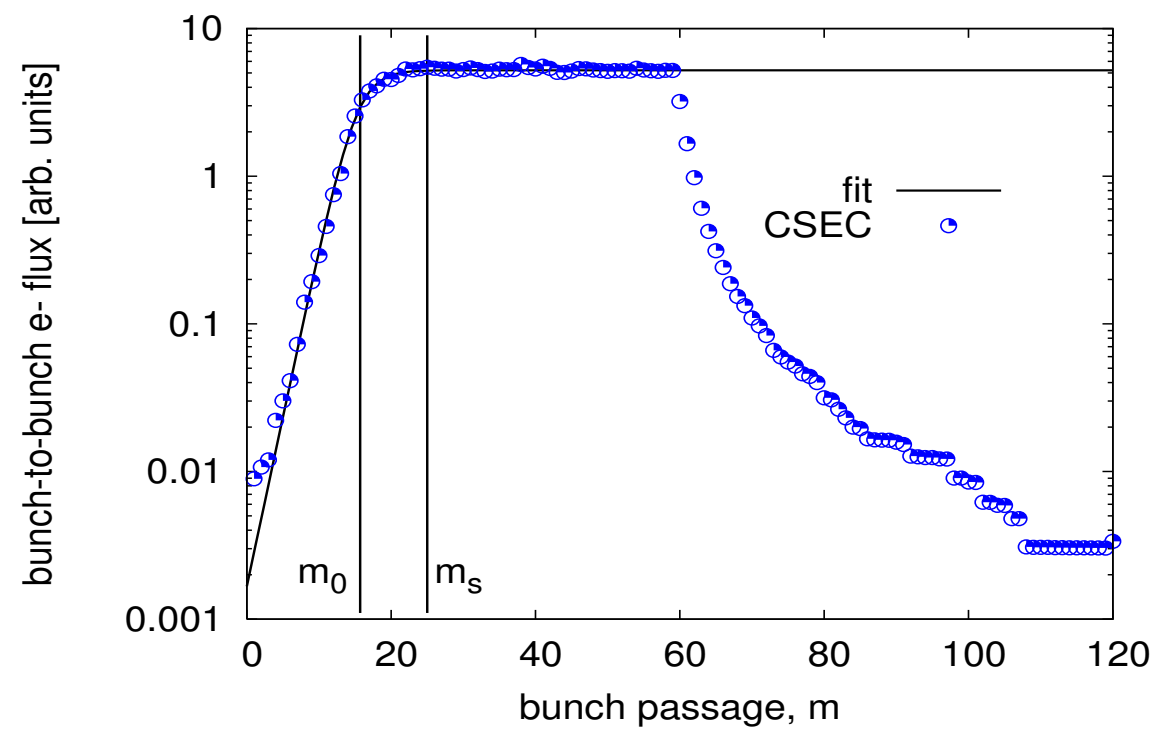

Figure 6.1: Average bunch-to-bunch electron flux evolution in a RHIC beam pipe after injection of 110 bunches spaced $107 \mathrm{~ns}$ apart during a RHIC revolution. With this bunch spacing, a RHIC revolution is equivalent to 120 bunch passages. A saturation level is reached after about 25 bunches.

2. For $m \gg m_{0}$, (and $m / B \gg 1$, as it is seen a posteriori) the factor $e^{\left(m-m_{0}\right) / B} \gg 1$, and the flux is

$$
\phi(m)=\phi_{s} \frac{e^{\left(m-m_{0}\right) / B}}{1+e^{\left(m-m_{0}\right) / B}} \approx \phi_{s}
$$

while the pressure becomes

$$
P(m)=P_{0}+A_{P} \ln \left(1+e^{\left(m-m_{0}\right) / B}\right) \approx P_{0}+A_{P}\left(m-m_{0}\right) / B .
$$

As it corresponds to the integral of a constant electron flux (Eq. 6.9), the pressure $P(m)$ (Eq. 6.10) exhibits a linear dependence on the bunch passage $m$.

Consistent with these two regimes, the flux in Fig. 6.1 first shows exponential growth (for $0<m<m_{0}$ ), followed by a constant regime due to the saturation effects (for $m \gg m_{0}$ ). Next, it will be shown that the experimental data (both for the electron flux and the pressure) exhibits these two regimes. For this, it is interesting to note that after $m_{s}>m_{0}$ bunches are injected, the bunch-to-bunch electron flux reaches a saturated value until the abort gap is reached. For the example in Fig. $6.1, m_{0}=16$ and saturation occurs at $m_{s}=25$.

\subsection{Experimental data}

An interesting event occurred during fill \#3460 (April 2003), when 110 bunches spaced by 107 ns with an average bunch intensity of $N_{b}=8 \times 10^{10}$ protons were injected into the RHIC 
ring. The top plot in Fig. 6.2 shows the time evolution of the pressure at a gauge $8 \mathrm{~m}$ away from the ED (red line, right vertical axis), and the electron signal averaged over one turn (black dots, left vertical axis). This is calculated averaging each of the one-turn snapshots taken by the ED, as shown in Chapter 5 - Eq. 5.9. Figure 4.7 shows an ED snapshot during this fill.

The sampling rate in Fig. 6.2 is $1 \mathrm{~Hz}$ for the pressure, and $0.25 \mathrm{~Hz}$ for the ED readings. The beam injection is shown in the bottom plot. Note that injection is temporarily halted for about two minutes after 45 bunches $(12 \mathrm{~h} 12 \mathrm{~m})$. Injection resumed $(12 \mathrm{~h} 14 \mathrm{~m})$ and finally finished at $12 \mathrm{~h} 20 \mathrm{~m}$. As the beam decays, both the electron signal and the pressure signal decay at a similar rate. The electron flux $\phi=d I / d l$ is directly proportional to the voltage in the ED - Eq. 4.4. The linear relation between the pressure readings and the electron signal averaged over one turn is also confirmed in Chapter 5.
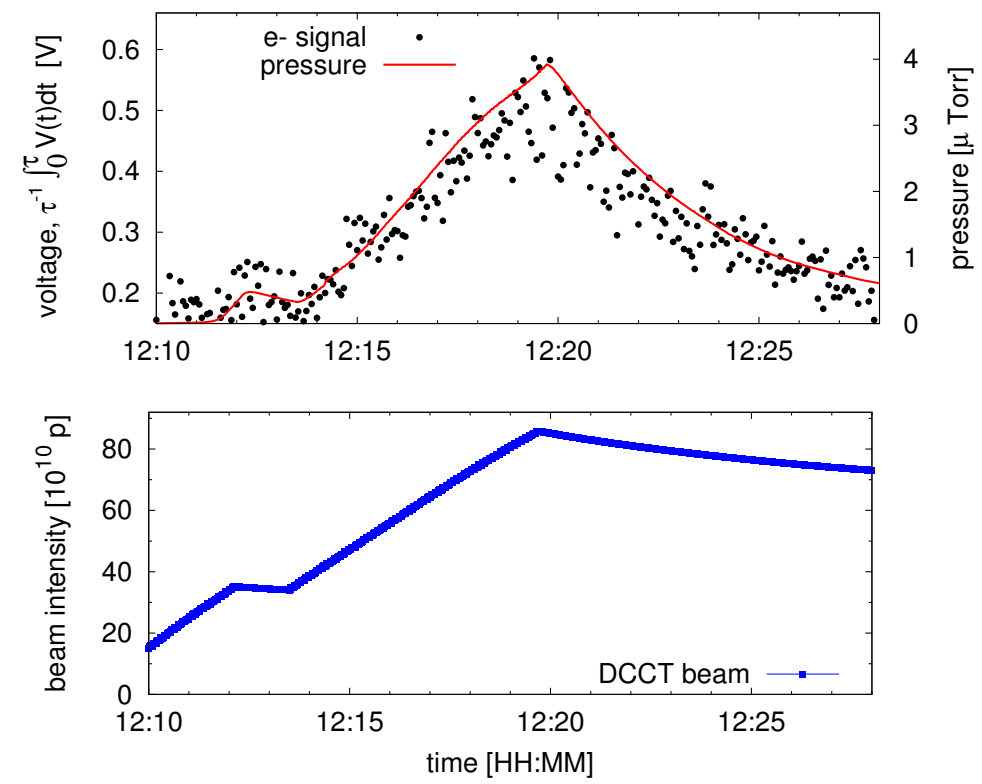

Figure 6.2: Dynamical pressure evolution and electron signal (top plot), as the blue beam is being injected (bottom plot) for fill 3460. Pressure and electron signal follow a similar evolution.

Equation 6.5 can be used to test pressure rise observations for electron cloud characteristics. Figure 6.3 shows the evolution of the pressure as a function of the DCCT beam during fill \#3460 at two different locations: in the BO2 section (blue dots), and in the baked stainless steel region BO10 (red points). The black line in both cases corresponds to a fit to the experimental data following Eq. 6.5. Unlike BO2, no EDs are installed in BO10. The good agreement of the fit with the experimental data supports the notion of electron clouds as the cause of the pressure rise also seen at BO10. Note that the beam intensity is directly proportional to the number of injected bunches $m$ (shown in the top horizontal axis in Fig. 6.3). The two different regimes corresponding to Eqs. 6.8 and 6.10 are noticeable for both BO2 and BO10. 


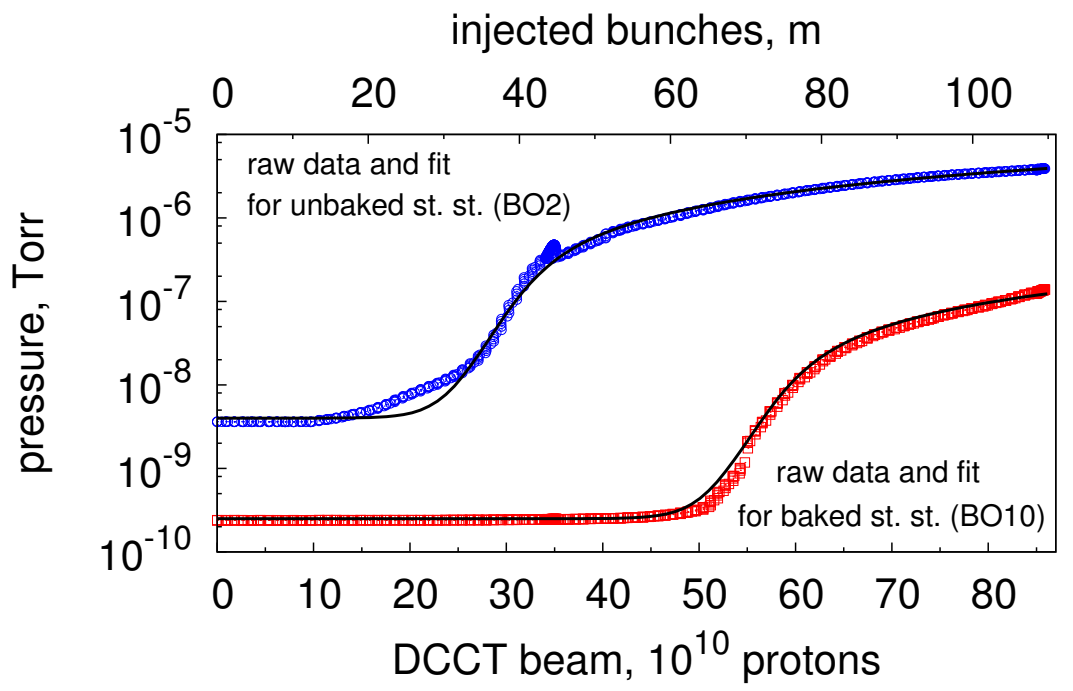

Figure 6.3: Pressure dependence as a function of the DCCT beam intensity and/or number of injected bunches (top horizontal axis) at two different regions. The red points correspond to the raw data at $\mathrm{BO} 2$, the blue points at BO10. The black line correspond to the fit following Eq. 6.5. Both cases show two different regimes, corresponding to a first exponential growth and a second linear regime.

For BO2, between beam intensities from 0 to $\approx 35 \times 10^{10}$ protons, the pressure grows exponentially two orders of magnitude (from $4 \times 10^{-9}$ to $5 \times 10^{-7}$ Torr). As mentioned earlier, at this point (at $12 \mathrm{~h} 12 \mathrm{~m}$ in Fig. 6.2), the injection was temporarly halted. This approximately coincides with the time at which the flux into the wall reaches a saturated value (see Fig. 4.7). After that, the pressure shows the expected linear dependence when $m>m_{s}>m_{0}$. The pressure stops growing after bunch 110 is finally injected. The fit has some discrepancy between 15 and $25 \times 10^{10}$ protons, probably due to the irregular bunch intensity, and/or the coexistance of other dynamic pressure effects, which are not considered in the fit. The fit results are shown in Table 6.1. It is surprising that the factor $B$ is similar for both cases. Both Table 6.1 and Fig. 6.3 show that fewer bunches are required to trigger electron clouds in unbaked surfaces $\left(m_{0}=37.5\right.$ vs 72.5$)$, and that pressure rises are around two orders of magnitude lower $\left(A_{P}=3 \times 10^{-7} / 9 \times 10^{-9} \approx 30\right)$. From Eqs. 6.5 and 6.6 , this can be due to lower electron fluxes $\phi_{s}$, lower electron desorption coefficients $\eta_{e}$, or both.

Overall, the agreement between the experimental data and the fit shows that electron clouds are responsible for the pressure rise during a beam injection. This is very useful when the electron flux remains below the noise level of the electron detector, or for regions where no electron detectors are installed. If the saturated level is reached and the pressure follows the linear regime, Eq. 6.5 can be used to estimate the maximum pressure a bunch train of $M$ bunches will produce just by injecting $m>m_{s}$ bunches.

Note that if the combination of bunch intensity, bunch length, secondary electron yield, 
Table 6.1: Fit results for Fig. 6.3. Results for the unbaked surface at BO10 show a smaller pressure rise, and a slower rise time (given by the larger $m_{0}$ ).

\begin{tabular}{lcc|c}
\hline \hline parameter & unit & $\begin{array}{c}\text { BO2 } \\
\text { value }\end{array}$ & $\begin{array}{c}\text { BO10 } \\
\text { value }\end{array}$ \\
\hline$m_{0}$ & $\ldots$ & 37.5 & 72.5 \\
$\mathrm{~B}$ & $\ldots$ & 2.0 & 2.1 \\
$A_{P}$ & torr & $3 \times 10^{-7}$ & $9 \times 10^{-9}$ \\
$P_{0}$ & torr & $3.6 \times 10^{-9}$ & $2.5 \times 10^{-10}$ \\
\hline \hline
\end{tabular}

electron desorption coefficient, et cetera, produces a pressure rise above the vacuum limit $\left(\approx 10^{-5}\right.$ Torr [68]), beam injection has to be interrupted. The analysis following the pressure as a function of the injected bunches is easily performed at RHIC because bunches can be injected one by one. At the SPS $[39,40,58]$ the LHC beam is injected in batches of 72 bunches, and therefore it is difficult to observe behaviour as shown in Fig. 6.3.

\subsection{Benchmarking simulations with experimental data}

It is worth comparing the output of the existing computer codes for electron cloud simulations with experimental data to check whether the observations can be reproduced with the current status of the codes. I tried to reproduce the electron signal behaviour obtained with the ED data from fill \#3460, whose characteristics are listed in the first part of Table 6.2. Figure 6.4 (left) shows, as an example, two snapshots of the electron cloud signal collected in the ED (top plot) and the bunch intensity (bottom plot) as read by the WCM. Between $t=1.8$ and $3.8 \mu \mathrm{s}$, the bunch intensity decreases from $8 \times 10^{10}$ to about $5.5 \times 10^{10}$ protons/bunch, causing the electron signal to interrupt its build up. In the snapshot at 12:19:52 the signal stays more or less constant. At 12:20:00 a slightly decrease is noticeable. This is arguably related to large ED noise. Two quantities are reproduced using CSEC: the peak to peak signal (which remains acceptably constant), and the decay time after the last bunch. Another characteristic is the growth time. However, because of the irregular bunch intensities, this is not a very reliable observable from the experimental data. The evolution of both quantities throughout the fill is analyzed in the following.

\subsubsection{Experimental values}

The left hand side plot in Fig. 6.5 shows the evolution of the peak to peak value observed in the ED assuming a transparency of 5\%. The ED at BO2 has a low frequency cut-off $f_{\text {low }}=32 \mathrm{kHz}$. Note that after the trigger of the electron cloud (slightly before 12:12:00), the flux stays more or less constant ranging between $\approx 2.5$ and $\approx 3.5 \mu \mathrm{A} / \mathrm{cm}^{2}$. However, two noise sources are present. In the absence of a multipacting (before 12:12), the noise level induced by the beam is about $1 \mu \mathrm{A} / \mathrm{cm}^{2}$. Secondly, the lack of knowledge of the electron energy spectrum indicates that the effective transparency can change by about a factor of 

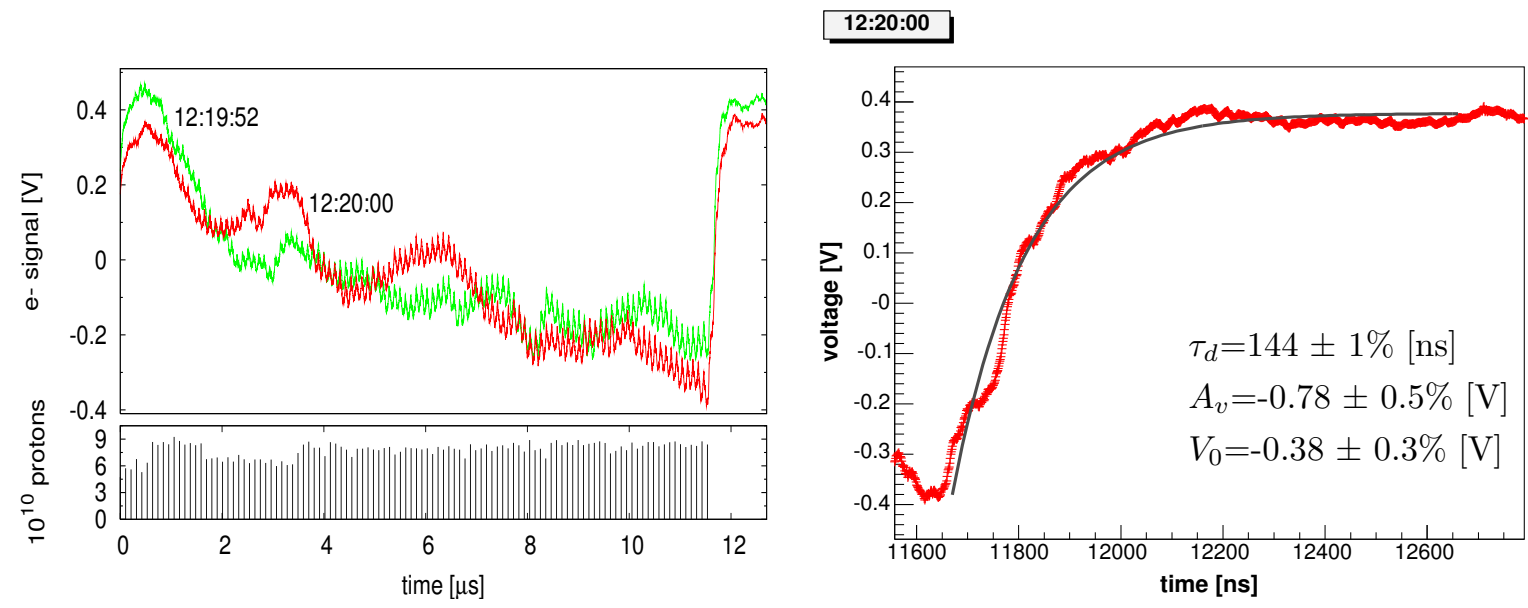

Figure 6.4: Left. Two ED snapshots only 4 seconds apart (top plot) and bunch intensity (bottom plot) for a RHIC revolution $(12.8 \mu \mathrm{s})$ during fill 3460 . Note the oscillations along the signal due to the ED noise. Right. Example of the fit using Eq. 6.11 to the decay of the electron flux after the last bunch has passed by.

2 (see Fig. 4.11). All in all, it is assumed that the saturated value is reproduced within a factor of 2 .
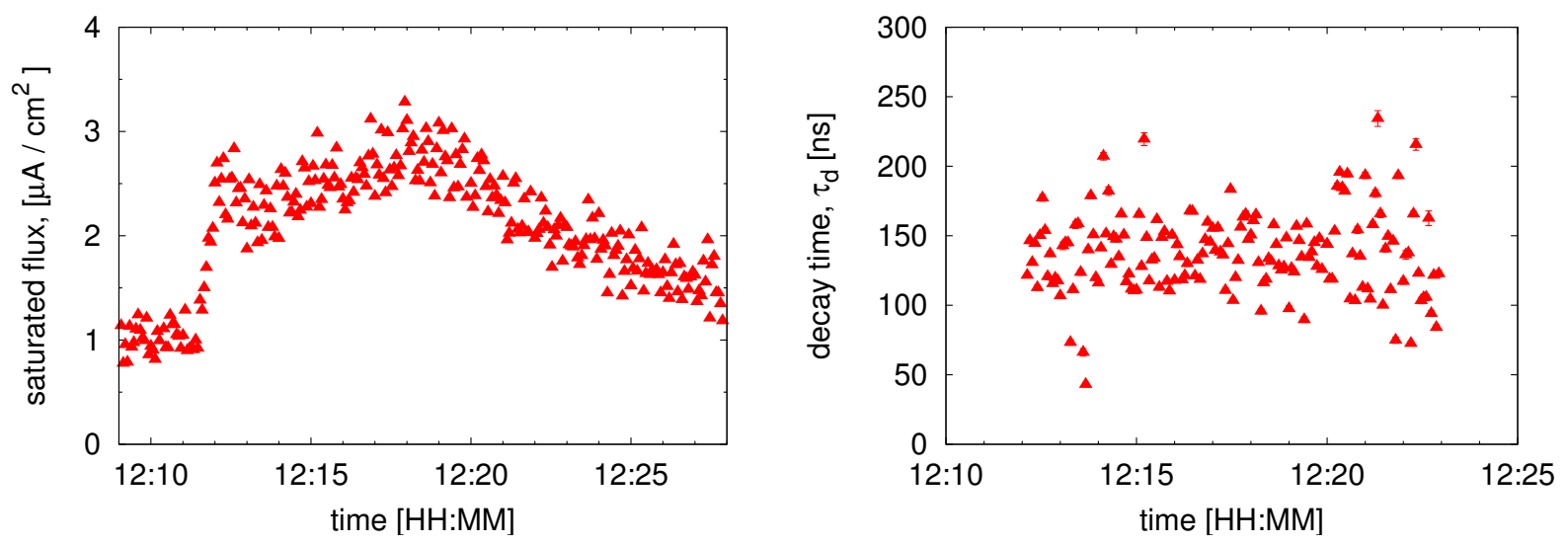

Figure 6.5: Evolution of the saturated flux (left) and decay time (right) during fill \#3460.

The right hand side plot in Fig. 6.4 shows an example of the fit to the experimental values for the snapshot at 12:20:00. The signal in the electron detector decays after the last bunch passage in the form:

$$
V(t)=A_{v} e^{-\left(t-t_{M}\right) / \tau_{d}}-V_{0},
$$

where the offset $V_{0}$ is produced by the electronics of the system, $A_{v}$ is a fitting parameter, and $\tau_{d}$ indicates the decay time. Appendix B shows that the ideal evolution of the cloud density shows two exponential regimes. In this case, the decay time obtained using the ED 
refers to the first regime, since the second regime shows very low energy fluxes and very low energy electrons, which are hardly distinguishable from the ED noise. Since $\tau_{d}$ is in the order of some hundreds of ns, this is not affected by the signal differentiation (low frequency cut off is $32 \mathrm{kHz}$ ). The time $t_{M}$ corresponds to the last bunch passage, and it is obtained from the WCM data. This fit is applied to each of the snapshots during fill \#3460. The evolution of the calculated time decay throughout the fill is shown in Fig. 6.5, right. The fitting is only performed for those snapshots showing a clear signal, between 12:12:00 and 12:23:00. The histogram in Fig. 6.6 shows the average and rms values for the decay time:

$$
\tau_{d}=140 \pm 30 \mathrm{~ns} .
$$

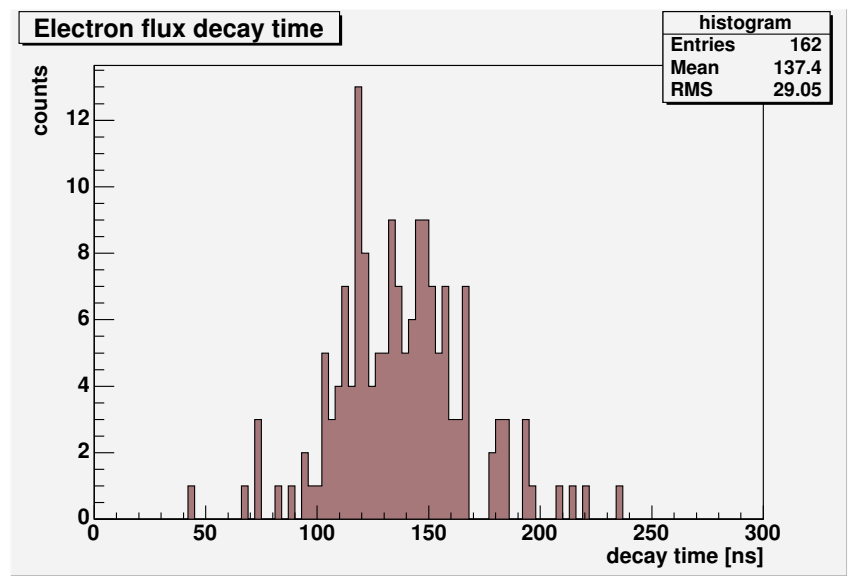

Figure 6.6: Histogram with the calculated decay times.

\subsubsection{Simulation results}

As seen in Section 3.3 one of the main uncertainties in the electron cloud simulation codes stems from the Secondary Electron Yield parameterization. This involves no less than six parameters (see Eqs. 3.22 and 3.23). The electron cloud formation is sensitive to many of them. It is interesting to fix the wall surface parameters to values similar to those obtained in the literature [46], and slightly sweep the most significant ones, $\delta_{\max }$ and $\delta_{0}$, comparing the saturated flux and the decay time with those obtained experimentally. These values, together with the beam conditions, are introduced as input parameters of the CSEC code, and are shown in Table 6.2. The code is modified to be able to reproduce the irregular bunch intensities along the bunch train, as observed in Fig. 6.4.

The left hand side plot in Fig. 6.7 shows three examples of the evolution of the simulated electron flux to the wall for $\delta_{\max }=1.7,1.9$, and 2.1. The data is numerically smoothed using a $10 \mathrm{MHz}$ filter, to avoid the electron bunch oscillations and reproduce the experimental data. Note that between 2 and $4 \mu s$ the build up is interrupted due to the lower bunch intensities, as it is observed in the experimental data (see Fig. 6.4, left). Figure 6.7, right, shows the decay time fit for the case corresponding to $\delta_{0}=0.6$ and $\delta_{\max }=2.0$. Since the decay of 
Table 6.2: List of input parameters for the electron cloud CSEC simulations.

\begin{tabular}{|c|c|c|c|}
\hline parameter & symbol & unit & value \\
\hline bunch spacing & $s_{b}$ & ns & 107 \\
\hline number of bunches & $\ldots$ & $\ldots$ & 110 \\
\hline pipe radius & $b$ & $\mathrm{~mm}$ & 60 \\
\hline number of turns & $\ldots$ & $\ldots$ & 2 \\
\hline full bunch length & $\sigma_{l}$ & ns & 15 \\
\hline bunch population & $N_{b}$ & $10^{10}$ protons & $5.5-8.5$ \\
\hline rms beam radius & $\sigma_{r}$ & $\mathrm{~mm}$ & 2.4 \\
\hline reflection probability for $E \rightarrow 0$ & $\delta_{0}$ & $\ldots$ & scan $[0.5-0.7]$ \\
\hline maximum SEY & $\delta_{\max }$ & $\ldots$ & $\operatorname{scan}[1.6-2.4]$ \\
\hline reflection probability for $E \rightarrow \infty$ & $\delta_{\infty}$ & $\ldots$ & 0.2 \\
\hline energy at maximum SEY & $E_{\max }$ & $\mathrm{eV}$ & 300 \\
\hline reflection energy & $E_{r}$ & $\mathrm{eV}$ & 60 \\
\hline energy of secondary emitted electrons & $E_{\mathrm{sec}}$ & $\mathrm{eV}$ & 8.9 \\
\hline distribution width of secondary electrons & $\sigma_{\mathrm{sec}}$ & $\mathrm{eV}$ & 4.5 \\
\hline
\end{tabular}

an electron cloud shows a combination of two exponential decays, the fit is only performed for $400 \mathrm{~ns}$ after the last bunch passage. See Appendix B for an illustration of these two regime decays. In the first, the cloud decays quickly due to the space charge effects and the reminiscences of the bunch passage. In the second, the electrons move slowly and their dissipation rate depends mainly on the elastic reflection probability of the chamber surface for these low energies (i.e., parameter $\delta_{0}$ in Eq. 3.23).
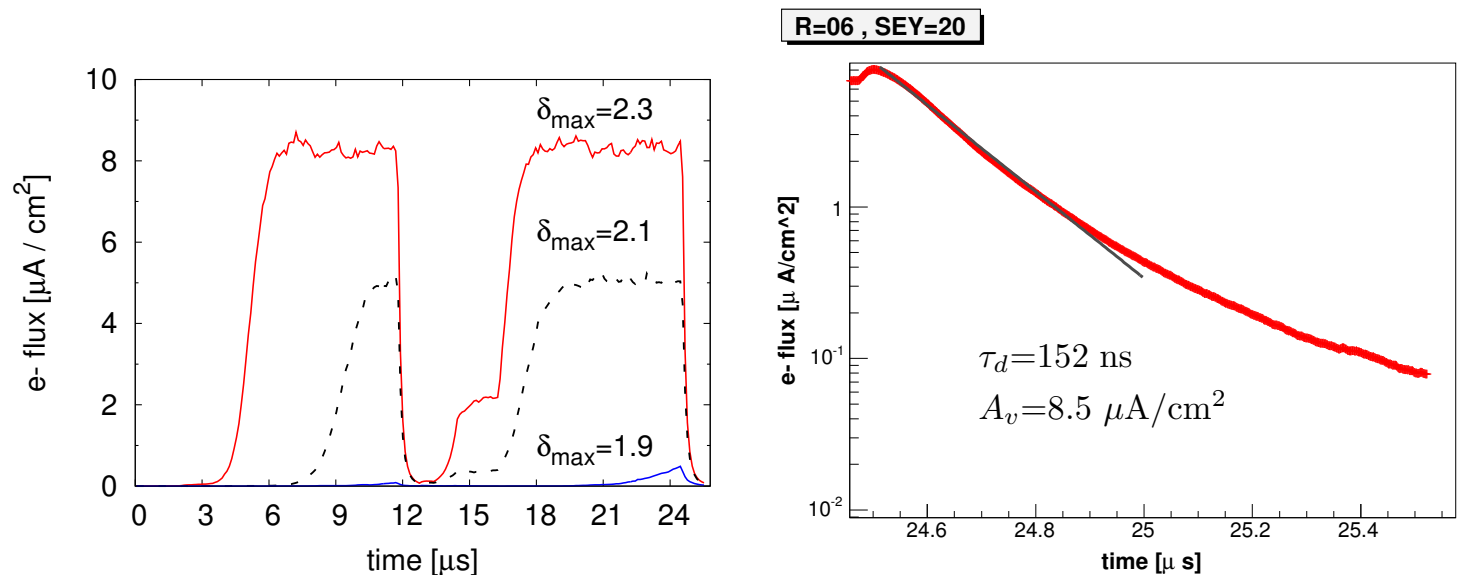

Figure 6.7: The left hand side plot shows the evolution of the electron flux as a function of time for three different $\delta_{\max }\left(1.9,2.1\right.$, and 2.3), while keeping $\delta_{0}=0.6$. The right hand side plot depicts an example of the fit (black line) to the simulation results during $400 \mathrm{~ns}$ after the last bunch for $\delta_{\max }=2.0, \delta_{0}=0.6$. The saturated flux and the decay time are compared with the values of the experimental data (Fig. 6.5). 
Figure 6.8 shows the peak electron flux in the simulation as a function of the maximum value of the secondary emission yield, $\delta_{\max }$ for three different values of $\delta_{0}: \delta_{0}=0.5$ (red trace), $\delta_{0}=0.6$ (black trace), and $\delta_{0}=0.7$ (blue trace). The left hand side plot in Fig. 6.8 shows the calculated decay times for the same set of $\delta_{0}$, and $\delta_{\max }$. Note that for $\delta_{\max }<1.9$, the calculated decay times (from CSEC simulations) are larger than those measured experimentally (see Fig. 6.6). For an easy comparison with the experimental values, these have been marked with a horizontal bold line in Fig. 6.8, while the limits of the error margin are marked with two dashed horizontal lines.

Combinations compatible with both the experimental saturated fluxes and the decay times Fig. 6.5 are listed in Table 6.3. Good SEY parameterizations combine $\delta_{0} \in[0.5,0.6]$, and $\delta_{\max } \in[2.0,2.3]$. Laboratory measurements in surface physics literature for stainless steel keep $\delta_{\max }$ around $2.1[46,50]$. Thus, the conclusion is that for the RHIC unbaked stainless steel surfaces, CSEC optimally reproduces the observations if $\delta_{0} \approx 0.55$, and $\delta_{\max } \approx 2.1$.

However, it should be noted that other codes, using different SEY parameterizations, might not agree with these values. It is interesting to observe that in this range of parameters, the decay time using CSEC during the first exponential decay is almost independent of $\delta_{0}$ (the reflection probability for electron energies $E \rightarrow 0$ ).

Table 6.3: Combination of SEY parameters whose CSEC output is compatible with the experimental results. The rest of the SEY parameters are listed in Table 6.2.

\begin{tabular}{|c|c|}
\hline \hline reflectivity at low energy & maximum secondary electron yield \\
\hline$\delta_{0}$ & $\delta_{\max }$ \\
\hline 0.5 & $2.2<\delta_{\max }<2.5$ \\
0.6 & $1.9<\delta_{\max }<2.3$ \\
0.7 & $1.5<\delta_{\max }<1.9$ \\
\hline \hline
\end{tabular}
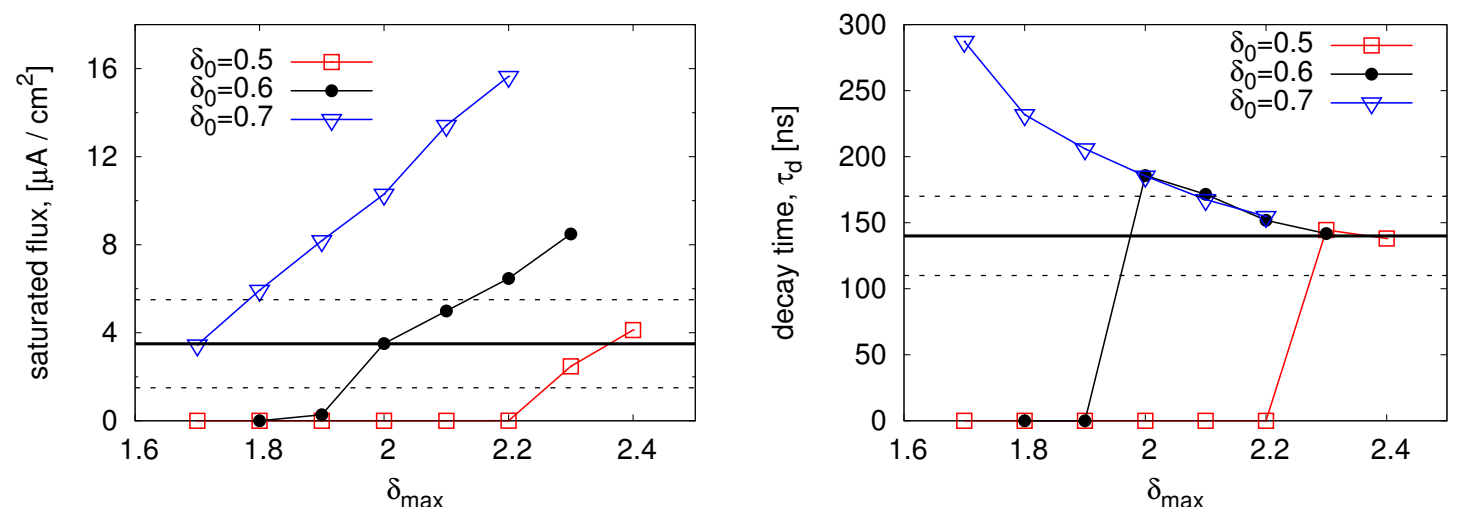

Figure 6.8: Saturated flux (left) and time decays (right) calculated after CSEC simulations as a function of the maximum secondary emission yield, $\delta_{\max }$, and for three different reflectivities: $\delta_{0}=0.5$ (blue triangles), $\delta_{0}=0.6$ (black dots), and $\delta_{0}=0.7$ (red squares). The experimental results are marked with the bold horizontal line $\left(\phi_{\text {sat }}=3.5 \mu \mathrm{A} / \mathrm{cm}^{2}\right.$ and $\left.\tau=140 \mathrm{~ns}\right)$. The experimental error range is indicated with dashed lines. 


\section{Chapter 7}

\section{Pressure rise in the experimental regions}

The beams for Physics fills that were injected into the RHIC rings during Runs 3 to 5 avoided the electron cloud conditions at injection that were seen in the previous Chapter 6 . Nonetheless, local pressure rises are observed when the deuteron, gold, or copper beams are accelerated, reaching a maximum when the beams cross the transition energy. Furthermore, the pressure usually rises again after "rebucketing", an RF gymnastics process by which the bunch length is shortened to about 5 ns full parabolic bunch length. These pressure rises are more pronounced in the Interaction Regions (IRs) where both beams travel in a common beam pipe. Figure 7.1 shows a typical pressure rise at IR10 (where the PHOBOS experiment is located) during a Physics fill in the Au-Au Run 4.
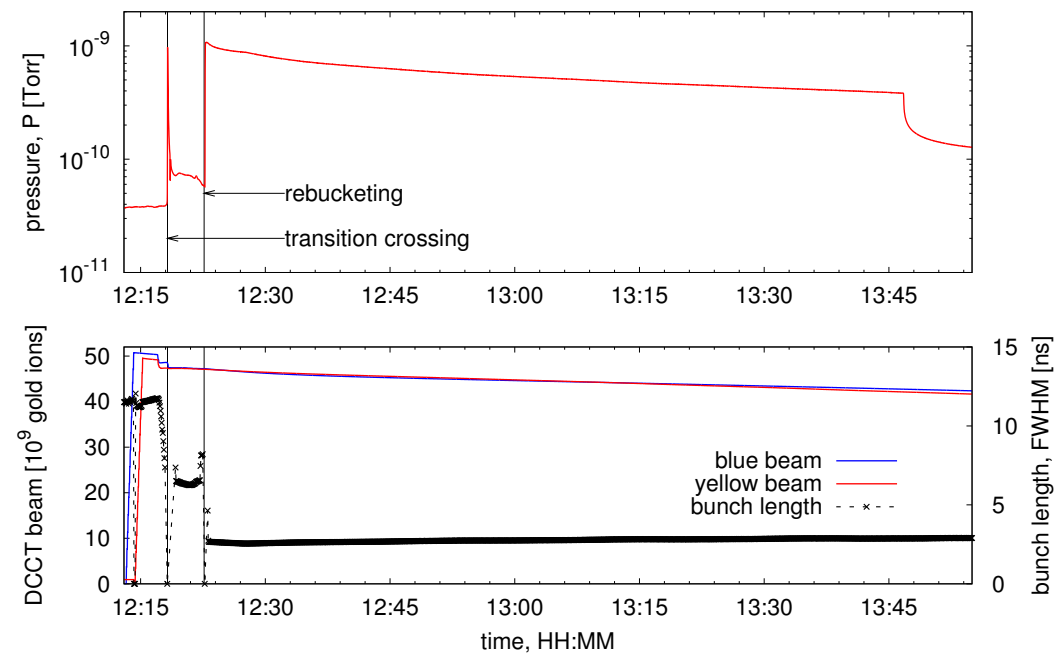

Figure 7.1: Typical intensities and bunch length (bottom), and pressure evolution (top) in IR10 during acceleration (left vertical line), transition crossing (middle vertical line), and rebucketing (right vertical line) during fill \#4535 in Run 4. 


\subsection{Two beams in a common beam pipe}

A common beam pipe refers to the vacuum chamber in the experimental regions, where the two beams (blue and yellow) travel in opposite directions. Depending on the longitudinal position along the beam pipe in the interaction regions, uneven (and compared with the bunch spacings in the single beam pipe, shorter) bunch spacings are created between the two beams. The situation is shown in Fig. 7.2 for two beams colliding at an arbitrary location $z_{0}$. Placed at a distance $\Delta z=z-z_{0}$ and still in the common beam pipe, a yellow bunch is separated by

$$
s_{b 1}=2|\Delta z|
$$

from a blue bunch, which in turn is at

$$
s_{b 2}=s_{b}-2|\Delta z|
$$

from the next yellow bunch passage, where $s_{b}$ is the bunch spacing for a single beam. The situation is symmetric for $-\Delta z$, swapping yellow bunches with blue bunches. Note that the distance between a blue and a yellow bunch passage, $s_{b 1}$, only depends on the distance, $\Delta z$, and it does not depend on the bunch spacing of a single beam $s_{b}$.
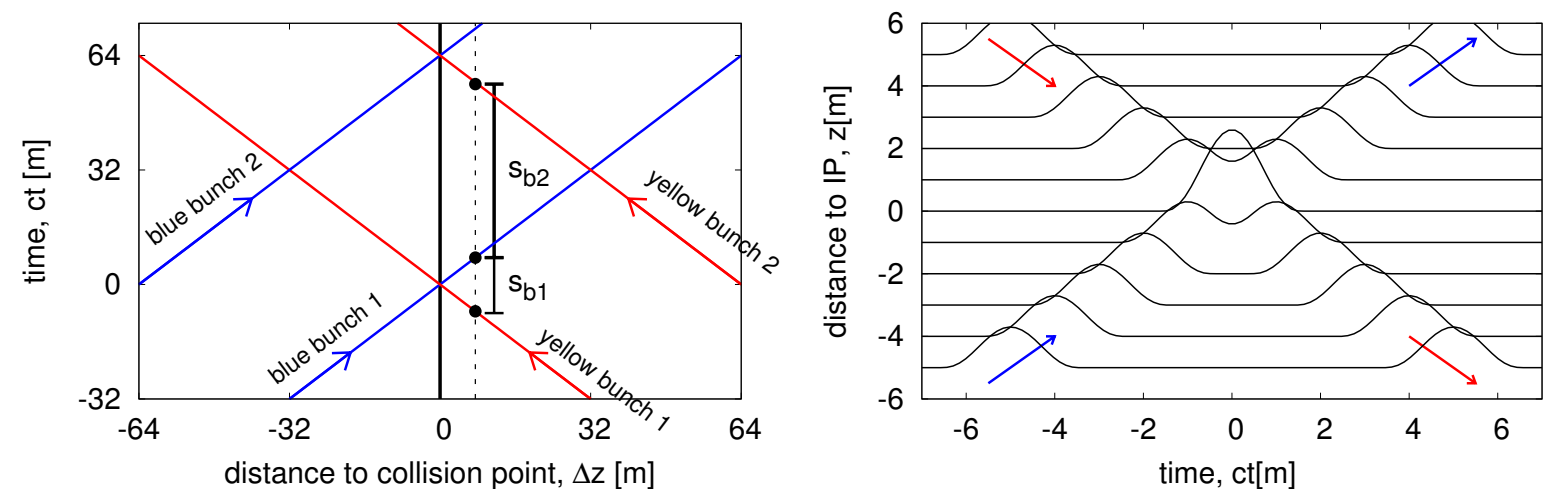

Figure 7.2: Left: Bunch spacings at a distance $z=7 \mathrm{~m}$ from the IP when the blue and yellow beams are colliding. The bunch spacing in a single beam pipe in this case is $s_{b}=64 \mathrm{~m}$ (214 ns). Right: Mountain range plot between $\pm 6 \mathrm{~m}$ from the IP considering a full width bunch profile of both blue and yellow beams $2 \sigma_{l}=1.8 \mathrm{~m}$, showing the different bunch profiles, spacings and intensities created between the two beams.

For simplicity, in the following it is assumed that $z_{0}$ is the middle point in the common beam pipe, or Interaction Point (IP). This is equivalent to say that the beams are cogged. The beams are anti-cogged during injection to avoid unwanted collisions, and they are cogged when the store conditions are reached to provide collisions to the experiments. When the beams are cogged, the bunch spacing at the IP is the same as for a single beam, but the bunch intensity doubles. For example, for a single beam bunch spacing of $64 \mathrm{~m}$ (214 ns) and 
placed at $7 \mathrm{~m}$ from the IP (dotted line at Fig. 7.2, left), the two different bunch spacings seen at this location are $s_{b 1}=14 \mathrm{~m}(47 \mathrm{~ns})$ and $s_{b 2}=50 \mathrm{~m}$ (167 ns). If the bunch spacing in a single beam is halved to $s_{b}=32 \mathrm{~m}, s_{b 1}=14 \mathrm{~m}$ as well, but $s_{b 2}=18 \mathrm{~m}$.

The situation is slightly different when the bunch length is comparable to the bunch spacing $s_{b 1}$. Consider the longitudinal bunch profile described by Eq. 3.16 with a total bunch length $2 \sigma_{l}$. At a distance $\left|z-z_{0}\right|<\sigma_{l}$, the bunch spacing $s_{b 1}<2 \sigma_{l}$, and the combination of the blue and yellow bunch traveling in the opposite directions is regarded as a single bunch with a "camel like" profile, as shown in Fig. 7.2, right. When $z=0$, the bunch intensity is doubled and the bunch spacing is the same as in a single beam pipe.

Thus, the presence of two beams in a common beam pipe not only produces different bunch spacings as a function of the location, $z$, but also different bunch profiles and bunch intensities. This produces an electron flux that varies as a function of the beam pipe location, $z$. When two beams are circulating in a common beam pipe in opposite directions, this combination of bunch spacings/length/intensities makes the IR more prone to electron clouds than the single beam pipes (as long as the rest of chamber parameters are similar - SEY, geometry, et cetera).

Figure 7.3 shows the saturated flux along the common beam pipe at IR10 using the parameters listed in Table 7.1. Consistent with similar studies (see Ref. [78]) the electron cloud builds up in the wings of the IRs, and decreases towards the middle of it, when the combination of bunch spacings is such that $s_{b 1}<2 \sigma_{l}$. Note that in the latter case, this is similar to a single beam pipe situation with bunch spacing $s_{b}$ and a bunch that is changed either in length, intensity, or both.
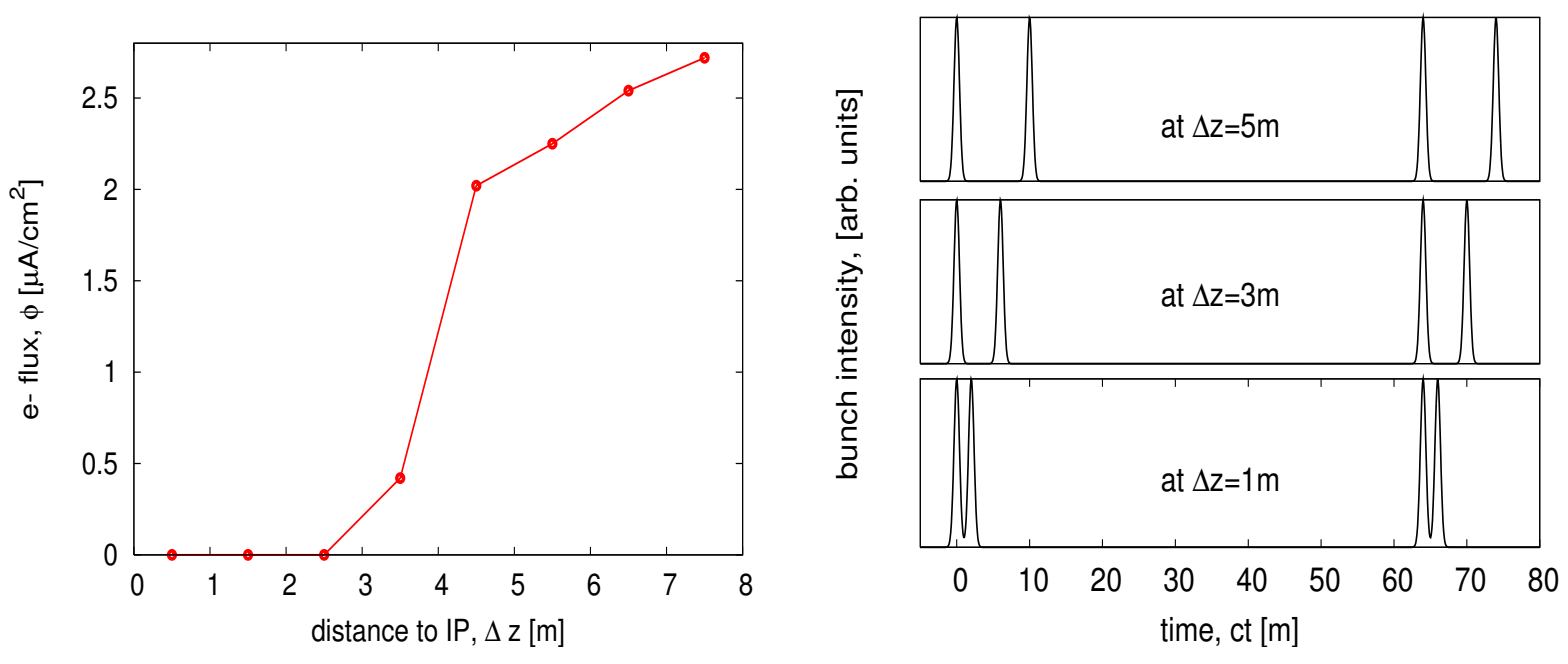

Figure 7.3: Left: Peak electron flux obtained using CSEC in a common beam pipe in RHIC during rebucketing conditions and as a function of the longitudinal beam pipe position. The flux decreases in the middle of the beam pipe (where the beams collide). Right: Three different bunch spacings/profiles corresponding to the simulations on the left hand side plot. 


\subsection{Experimental results}

Figure 7.4 shows an experimental snapshot of an electron cloud build up using the electron detector in the interaction region IR12 (see Fig. 5.3). During fill \#5201, 56 proton bunches per ring spaced by $s_{b}=64 \mathrm{~m}$ with an average bunch intensity of $8.4 \times 10^{10}$ protons/bunch were injected in the machine. Figure 5.7 shows the evolution of the pressure and electron detector (ED) flux in IR12 as the beam is injected.

The proton bunches do not cross the transition energy in RHIC, and their bunch length variation during the fill is not as significant as for the ion beams, but stays around $6 \mathrm{~ns}$ FWHM - see Fig. 5.7. Figure 7.4 illustrates how the electron cloud builds up when the two beams are present. In this particular snapshot, the beams are uncogged, that is to say, there is a relative phase offset of three buckets. According to this offset phase, the colliding position is at $z_{0}=12 \mathrm{~m}$ from the middle of the IR - outside the common beam pipe- so that parasitic collisions are avoided. The ED is located at the middle of the $\mathrm{IR}^{\dagger}, z=0$, so the two bunch spacings are, according to Eqs. 7.1 and 7.2,

$$
s_{b 1}=24 \mathrm{~m}, \text { and } s_{b 2}=40 \mathrm{~m} \text {. }
$$

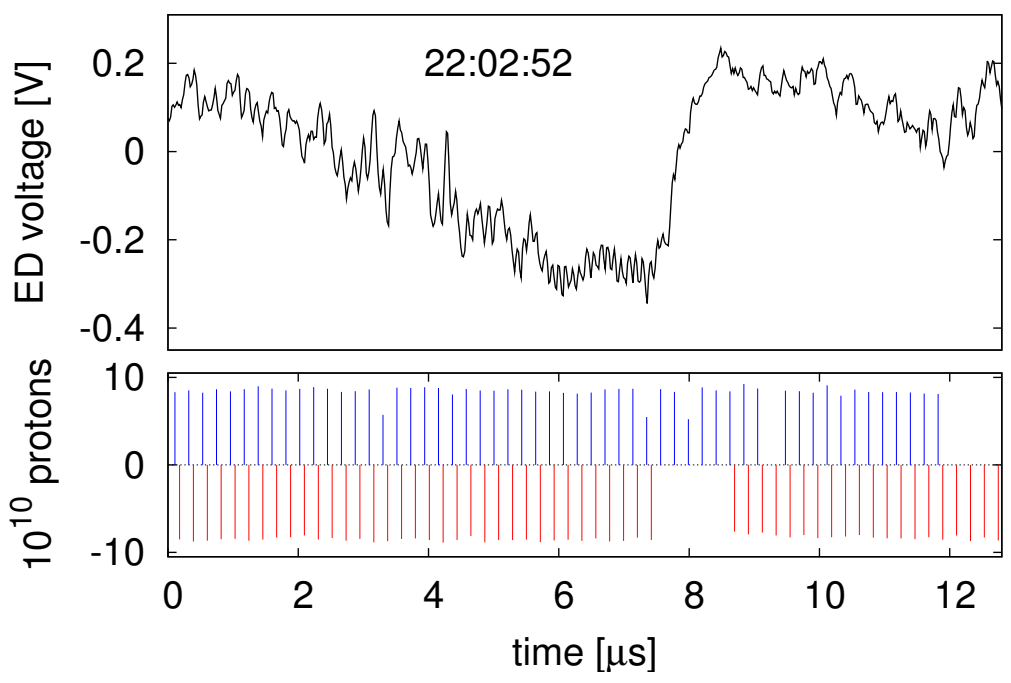

Figure 7.4: Electron detector snapshot at 22:02:52 (top plot) during one RHIC revolution period for fill \#5201 - see Fig. 5.7. The bottom plot shows the intensity of the blue and yellow bunches, which are depicted with a negative sign to represent their counter-circulating direction with respect to the blue bunches. The electron cloud is triggered when the two beams are present in the beam pipe, and it decays otherwise (in the presence of the blue or yellow abort gap).

The black trace in the top plot of Fig. 7.4 shows the signal in the ED, which is triggered with the first blue bunch passage. The blue pulses in the bottom plot correspond to the blue bunches. The red pulses represent the yellow bunch intensities, depicted with a negative sign

\footnotetext{
†The exact position is at $0.3 \mathrm{~m}$ from the middle point of the IR
} 
to show they are circulating in opposite direction of the blue bunches. The electron cloud saturates at approximately $6 \mu s$, and it decreases during the yellow abort gap passes through the IR (between 7.4 and $8.5 \mu \mathrm{s}$ ). After this time, the two beams coincide and the build up starts again now until the blue abort gap passes through the IR (at $\approx 11.8 \mu \mathrm{s})$, when the electron cloud decays again.

\subsubsection{Electron cloud decay in the interaction regions}

As performed in Section 6.4.1, the decay of the electron flux between 7.4 and $8.5 \mu s$ is fitted with Eq. 6.11. An example is shown in Fig. 7.5 (right), and it is carried out for all the ED snapshots taken during this fill. The results of these fits are shown in Fig. 7.5 (right). As opposed to the case of the single beam pipe in Fig. 6.5, the decay time is slower because blue bunches are present while the yellow abort gap occurs. Note the wide range of the decay time results in Fig. 7.5 (right). This is due to the difficulties in synchronizing the last yellow bunch passage, which shifts a lot during the acceleration and cogging processes (see Fig. 5.7). The final mean and rms values for the distribution in Fig. 7.5 (right) are:

$$
\tau_{d}=410 \pm 180 \mathrm{~ns},
$$

which represents almost a factor of 3 larger than the decay time calculated at the single beam pipes (see Eq. 6.12).
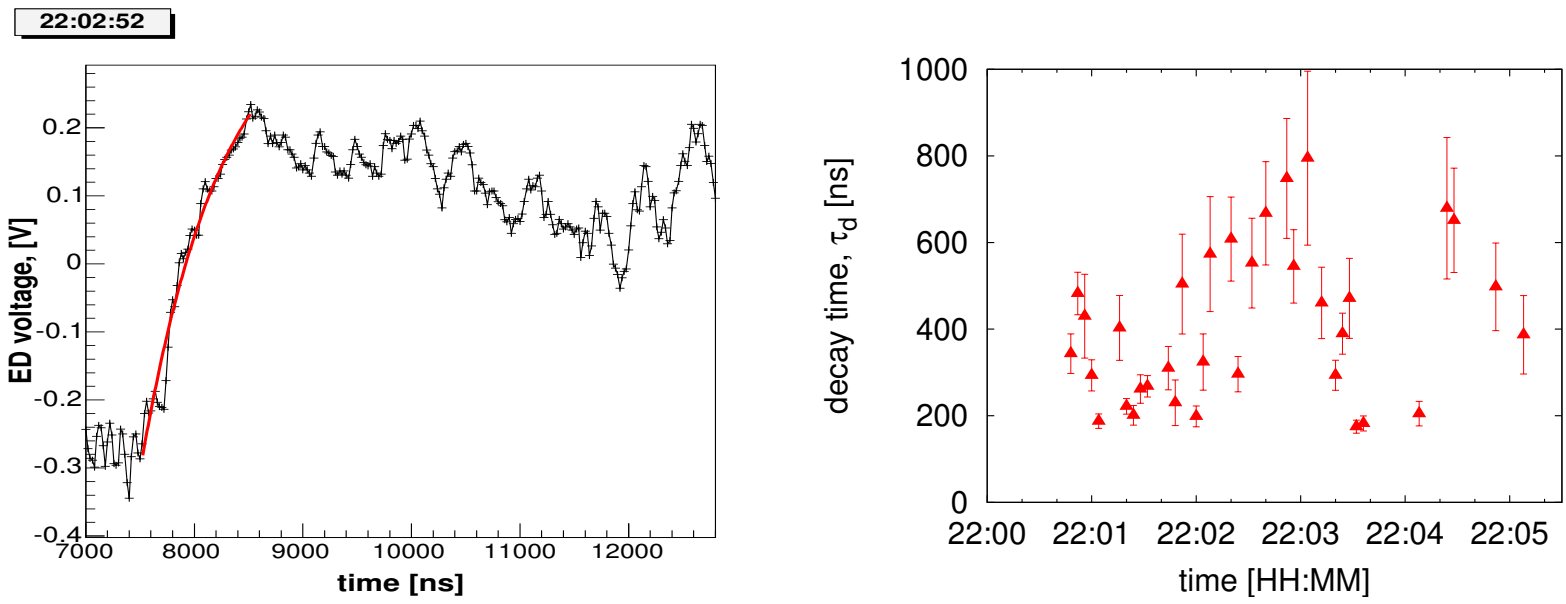

Figure 7.5: Left: Example of the fit to the decay of the electron flux after the last yellow bunch has passed by. This corresponds to the snapshot in Fig. 7.4. Right: Calculated decay times of the experimental ED snapshots along fill \# 5201, whose characteristics are seen in Fig. 5.7. The wide range of the values and error bar stems from the difficulties in synchronizing the yellow bunch passage during the acceleration and cogging processes. 


\subsection{Bunch length influence}

In Chapter 3 it is shown that the energy at which the electrons are accelerated towards the vacuum chamber due to the bunch passage in RHIC depends as $1 / \sigma_{z}$, being $\sigma_{z}$ the rms bunch length - see Fig. 3.2. Consistently, Fig. 7.1 suggests that the reduction of the bunch length is a key ingredient in the pressure rises at IR10 (PHOBOS).

The code CSEC is modified to reproduce the uneven bunch spacings/lengths/intensities created in a common beam pipe for two colliding beams. The situation at $7 \mathrm{~m}$ from the IP at IR10 is simulated modifying the SEY parameters to reproduce the observations in Fig. 7.1. Using the SEY parameters in Table C.1 for IR10 (Beryllium beam pipe), an electron cloud formation is found using CSEC for injection, upon transition crossing, and rebucketing conditions. However, since the PHOBOS beam pipes have not been opened to air since the RHIC commissioning, it is expected that the surface properties have been significantly affected by beam conditioning (for example, scrubbing by electron bombardment). Thus, the SEY parameters have been modified to reproduce the behaviour shown in Fig. 7.1: no multipacting under injection conditions (with bunch lengths of FWHM $\approx 12$ ns, and multipacting for transition/rebucketing conditions (with bunch lengths of FWHM $\approx 2.5 \mathrm{~ns}$ ). The final SEY parameterization listed in Table 7.1 (IR10 column) is consistent with similar simulations carried out for the PHOBOS characteristics [78].

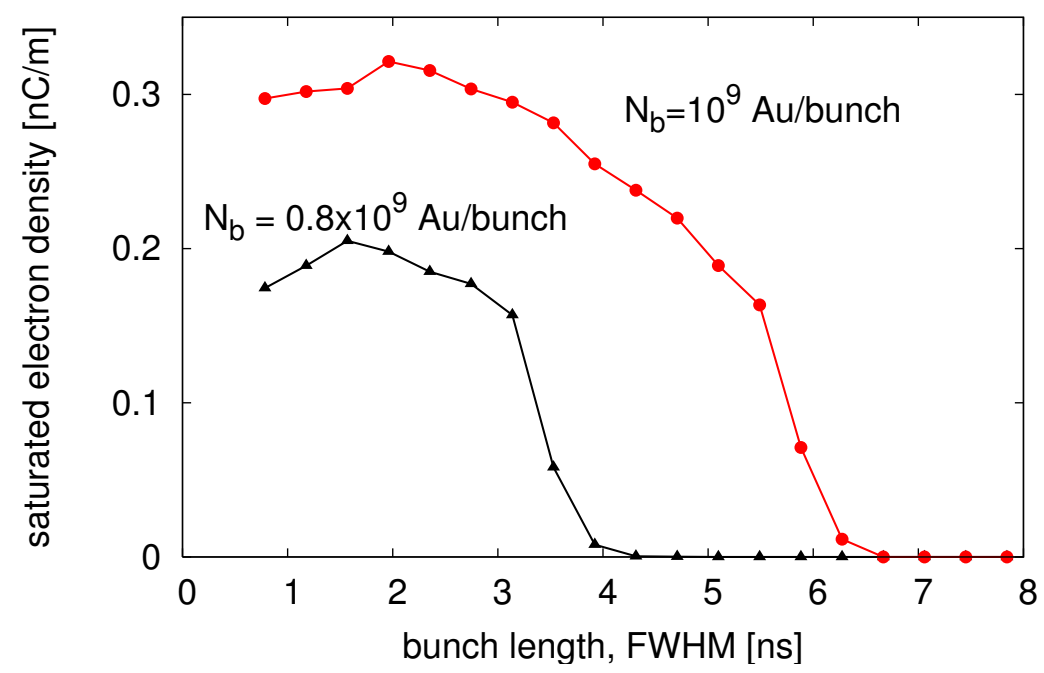

Figure 7.6: Effect of the bunch length for two different bunch intensities, $N_{b}=10^{9} \mathrm{Au}$ ions/bunch (red circles), and $N_{b}=0.8 \times 10^{9} \mathrm{Au}$ ions/bunch. Electron clouds are triggered by shortening bunches and increasing bunch populations.

The electron cloud density at $7 \mathrm{~m}$ from the IP in IR10 is simulated using CSEC for a bunch train of 56 bunches per ring. The total bunch length $2 \sigma_{l}$ is related to the FWHM as

$$
\mathrm{FWHM}=\frac{2 \sigma_{l}}{2.55}
$$


The saturated electron density as a function of the bunch length is depicted in Fig. 7.6 for two different bunch populations, $N_{b}=0.8 \times 10^{9} \mathrm{Au}$ ions/bunch (black triangles), and $N_{b}=10^{9} \mathrm{Au}$ ions/bunch (red points). Electron clouds are triggered when the bunch length is reduced. By using the SEY parameters listed in Table 7.1, electron clouds are formed when the FWHM $<7 \mathrm{~ns}$. However, note that Fig. 7.6 shows a piecewise continous transition between electron cloud "off" (zero saturated electron density), and "on" (nonzero saturated electron density). On the other hand, in Fig. 7.1, at about 13:46 the pressure abruptly decays while both the bunch intensity and length evolve smoothly. This behaviour, characteristic of first order phase transitions, is not qualitatively reproduced in Fig. 7.6 or other simulations for IR10 [78]. This was first addressed in Ref. [79] and is further developed in Chapter 11.

\subsection{Transition pressure rise}

An example of the pressure rise that occurs as the beams cross the transition energy is shown in Fig. 7.7. The pressure starts rising when the acceleration ramp begins, reaching a maximum upon transition crossing, first in IR12 (red line, top plot in Fig. 7.7) and later in IR10 (dashed line in the top plot in Fig. 7.7). After transition crossing, the pressure drops back. The pressure rise in most cases precedes, and sometimes coincides with visible beam loss (about $0.5 \%$ losses, corresponding typically to about $1 \times 10^{9} \mathrm{Au}$ ions). During Physics operation, the transition pressure rises are visible only in the warm regions, in the common beam pipes at IR10, IR12, and to lesser extent in IR4. In the common beam pipes of other interaction regions, as well as some other warm regions, pressure rises are only observable for very high intensities. These regions have the most appropriate conditions for electron clouds because of the combination of beam pipe material and geometry: IR12 is made of baked stainless steel, but it has a larger radius $-6 \mathrm{~cm}$, and IR10 is made of $12 \mathrm{~m}$ of Beryllium, which has the largest SEY among the materials used in the RHIC IRs - see Appendix C for a description of the geometry, material and the SEY at the different IRs.

An analisys of the transition pressure rise as a function of location, beam intensity, bunch intensity, beam loss, and fill pattern is performed in [80]. While pressure rises at injection and store could be directly related to electron clouds (see Chapter 6 and Ref. [78,81]), the source of the transition pressure rise was not so clear because:

- no electron detector (ED) signals could be observed prior to Run 5. Possibly, this was because of the high noise level in the ED. During beam experiments in Run 5, electron signals were clearly observed upon transition crossing.

- during Run 3, the pressure showed some dependence on the total intensity [82], and beam loss was suspected as a possible source for the release of gas molecules.

Next, it is shown how electron induced desorption, after an electron cloud formation, can explain the observed transition pressure rise. 


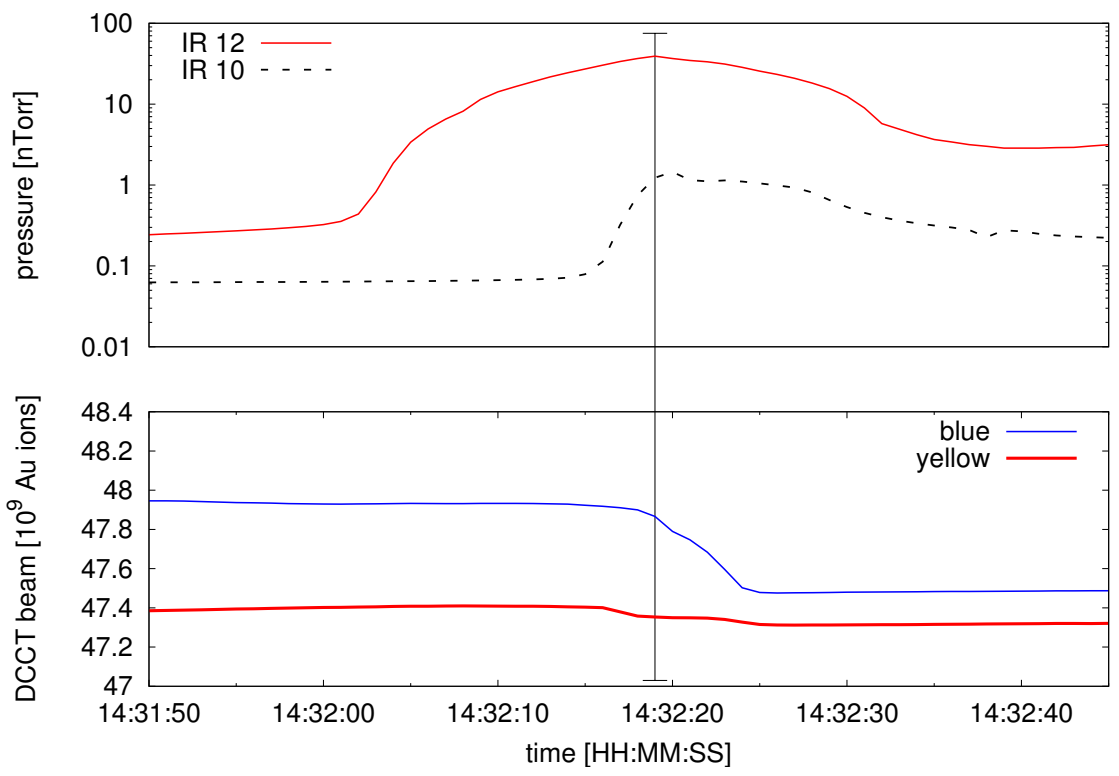

Figure 7.7: Typical intensities (bottom) and pressure evolution (top) in IR10 and IR12 during Run 4 when the beams cross the transition energy, indicated by the marker close to 12:32:20. The data correspond to fill \#4758.

\subsubsection{Transition pressure rise due to electron clouds}

Figure 7.8 shows the pressure in IR10 (left) and IR12 (right) when the beams cross the transition energy as a function of the total beam intensity (plots $\mathbf{a}$ and $\mathbf{b}$ ), and average bunch intensity (plots $\mathbf{c}$ and $\mathbf{d}$ ) for all fills during Run 4 in IR10 and IR12. The bunch intensity is the average of the blue and yellow ring intensities, before and after transition. The data show that the pressure rise is strongly beam intensity dependent (plots $\mathbf{a}$ and $\mathbf{b}$ ). Averaging the bunch intensity (plots $\mathbf{c}$ and $\mathbf{d}$ ) shows that the data is well separated into bunch patterns of 45,56, and 61 bunches per ring, showing that with more bunches (lower average bunch spacing), the pressure rise threshold is lower. This is consistent with the notion of electron clouds as the driving mechanism for this pressure rise.

\section{Simulation results}

However, the total beam intensity dependence needs further clarification. The electron cloud behavior in IR10 and IR12 for bunch patterns with 45, 56, and 61 bunches per ring is simulated using CSEC. A constant total intensity of $90 \times 10^{9} \mathrm{Au}$ ions is chosen, a value at which there seems to be no difference in the pressure rise for the different bunch patterns (see Fig. 7.8, bottom plots). The average bunch intensity changes accordingly (see Table 7.1). It is considered the bunch lengths at injection, transition, and store, with the simulation parameters listed in Tab. 7.1. The observation points are the locations close to a vacuum gauge, which are different in both IRs (Tab. 7.1). For simplicity, it is assumed that the abort 
$\operatorname{IR} 10$
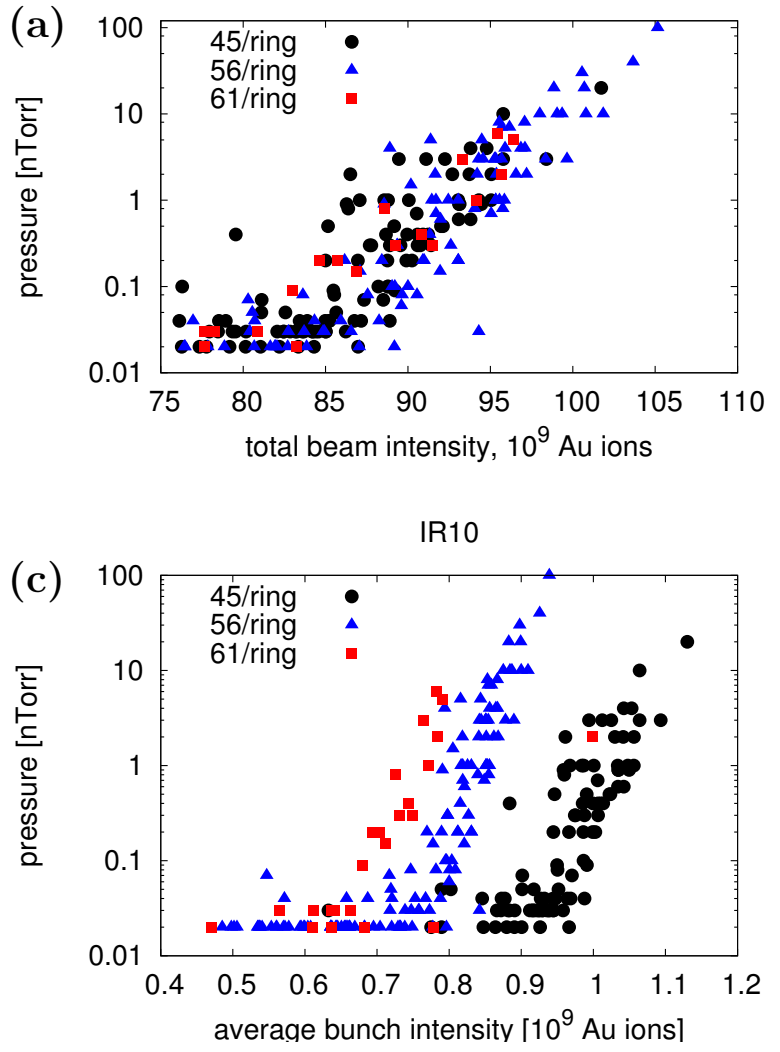

IR12

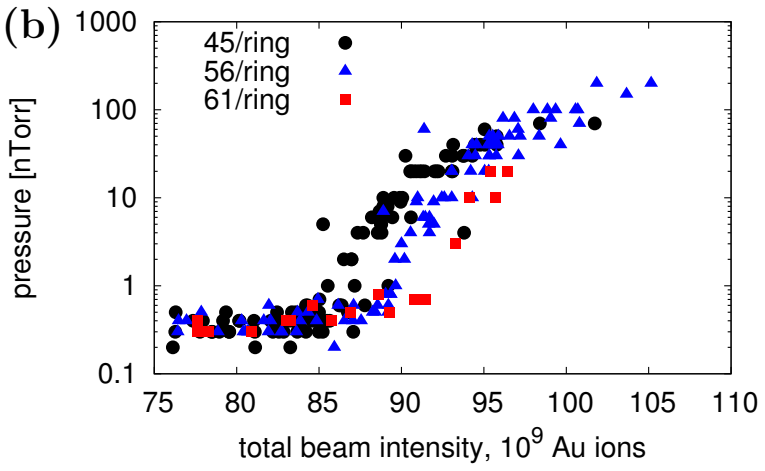

IR12

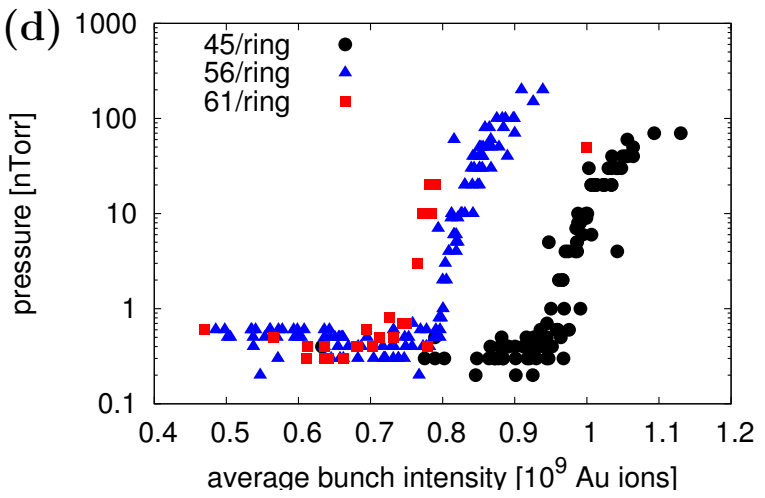

Figure 7.8: Transition pressure rise at IR10 (left column) and IR12 (right column), as a function of the total beam intensity (top plots, $\mathbf{a}$ and $\mathbf{b}$ ), and average bunch intensity (bottom plots, $\mathbf{c}$ and d). Total beam intensity refers to the sum of the blue and yellow intensities at transition. The bunch intensity is averaged over the blue and yellow ring intensities, and the values before and after transition. The data in the bottom plot is further separated into ramps with 45, 56, and 61 bunches/ring.

gaps coincide at both IRs.

The final SEY values listed in Tab. 7.1 were chosen to reproduce the observation that there is no electron cloud at injection with a full parabolic bunch length of $2 \sigma_{l}=17.5 \mathrm{~ns}$, and an electron cloud at transition $\left(2 \sigma_{l}=3.5 \mathrm{~ns}\right)$ and store $\left(2 \sigma_{l}=5 \mathrm{~ns}\right)$. As seen in Ref. [6, 47], there is a significant uncertainty in the SEY behavior as a function of the electron energy (especially at low energy) and the electron dose. Both dependencies have a strong influence in the studied cases. The surface parameters listed in Tab. 7.1 correspond to typical values found for stainless steel, OFHC and beryllium [46-48, 50,74].

In Fig. 7.9 the simulation results are shown for IR10. No electron cloud is observed for the injection conditions. For transition and store conditions, there are some variations in the electron flux into the wall when going from 61 to 56 to 45 bunches per ring. The energy of the cloud electrons increases monotonically when going to a smaller bunch number with larger 
Table 7.1: List of input parameters for electron cloud simulations at IR10 and IR12. The beam particles in all cases are gold ions $(\mathrm{Z}=79)$.

\begin{tabular}{|c|c|c|c|}
\hline parameter & unit & $\begin{array}{l}\text { IR10 } \\
\text { value }\end{array}$ & $\begin{array}{l}\text { IR12 } \\
\text { value }\end{array}$ \\
\hline bunch spacing & $\mathrm{ns}$ & \multirow{2}{*}{\multicolumn{2}{|c|}{$\begin{array}{c}324 / 216 / 108 \\
45 / 56 / 61\end{array}$}} \\
\hline bunches/ring & $\ldots$ & & \\
\hline single bunch population & $10^{9}$ & \multicolumn{2}{|c|}{$1.0 / 0.8 / 0.74$} \\
\hline single beam intensity & $10^{9}$ & \multicolumn{2}{|c|}{45} \\
\hline full bunch length & ns & \multicolumn{2}{|c|}{$17.5 / 3.5 / 5.0$} \\
\hline rms beam radius & $\mathrm{mm}$ & \multicolumn{2}{|c|}{2.0} \\
\hline pipe radius & $\mathrm{mm}$ & 36 & 60 \\
\hline distance from IP, $\Delta z$ & $\mathrm{~m}$ & 7.0 & 1.0 \\
\hline electrons generated/bunch & $\ldots$ & \multicolumn{2}{|c|}{20000} \\
\hline electron generation radius & $\mathrm{mm}$ & 36 & 60 \\
\hline longitudinal slices & per turn & \multicolumn{2}{|c|}{312000} \\
\hline macro-particles, initially & $\ldots$ & \multicolumn{2}{|c|}{25} \\
\hline smoothing length $d$ & $\mathrm{~mm}$ & \multicolumn{2}{|c|}{1.0} \\
\hline$\rho_{c e}$, initial & $\mathrm{pC} \cdot \mathrm{m}^{-1}$ & \multicolumn{2}{|c|}{0.16} \\
\hline$\delta_{\max }$ & $\ldots$ & 2.4 & 1.9 \\
\hline$E_{\max }$ & $\mathrm{eV}$ & 450 & 310 \\
\hline$\delta_{0}$ & $\ldots$ & 0.6 & 0.5 \\
\hline$\delta_{\infty}$ & $\ldots$ & \multicolumn{2}{|c|}{0.1} \\
\hline$E_{\text {reflect }}$ & $\mathrm{eV}$ & \multicolumn{2}{|c|}{60} \\
\hline$E_{\text {secondary }}$ & $\mathrm{eV}$ & \multicolumn{2}{|c|}{8.9} \\
\hline$P_{\text {rediffuse }}$ & $\cdots$ & \multicolumn{2}{|c|}{0.5} \\
\hline$\alpha_{P}$ & $\ldots$ & \multicolumn{2}{|c|}{1.0} \\
\hline
\end{tabular}

bunch intensity. Note that the shorter the bunch length, the larger the electron density and energy to the wall.

Figure 7.10 shows the results for IR12. Here too no electron cloud is observed for the injection conditions. For transition and store conditions, when going from 61 to 56 to 45 bunches per ring there is a small reduction in the electron flux into the wall. The difference in the electron energy for different fill patterns is more pronounced than at IR10. This is best seen in the maximum electron energy, which rises, for the transition case, from about $500 \mathrm{eV}$ for 61 bunches per ring to about $700 \mathrm{eV}$ for 45 bunches per ring. At IR10 the maximum electron energy is about $100 \mathrm{eV}$ lower.

An increase in the electron energy leads to a higher pressure rise if the desorption coefficient increases with the electron energy. In Ref. [70] molecular desorption coefficients are shown for electron energies between 20 to $300 \mathrm{eV}$. In this range they monotonically increase. No recent literature is found with measurements at higher energies, but it is possible that the desorption coefficients do not (or only slightly) increase further with higher electron energies [45]. 
Electron flux
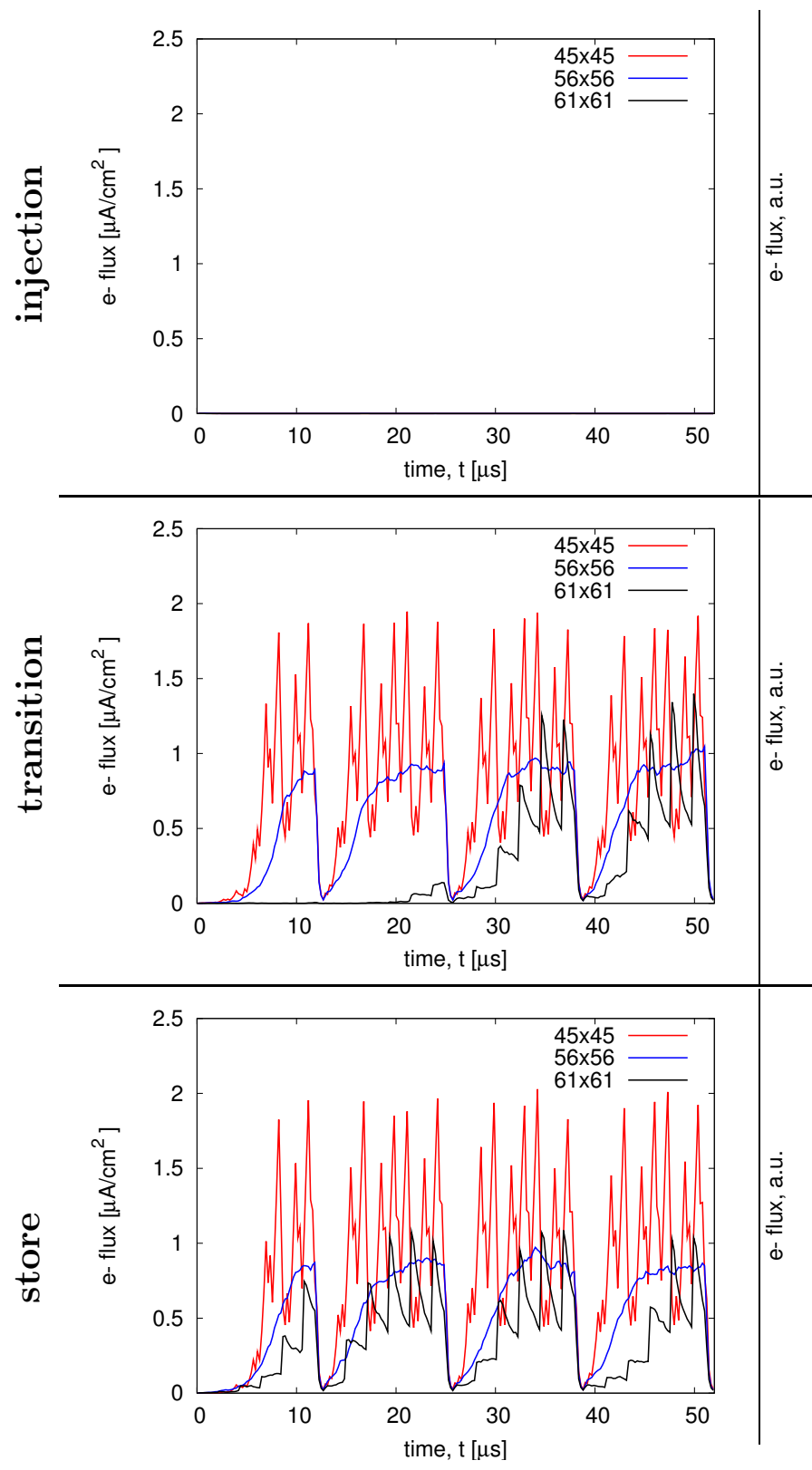

Energy spectrum
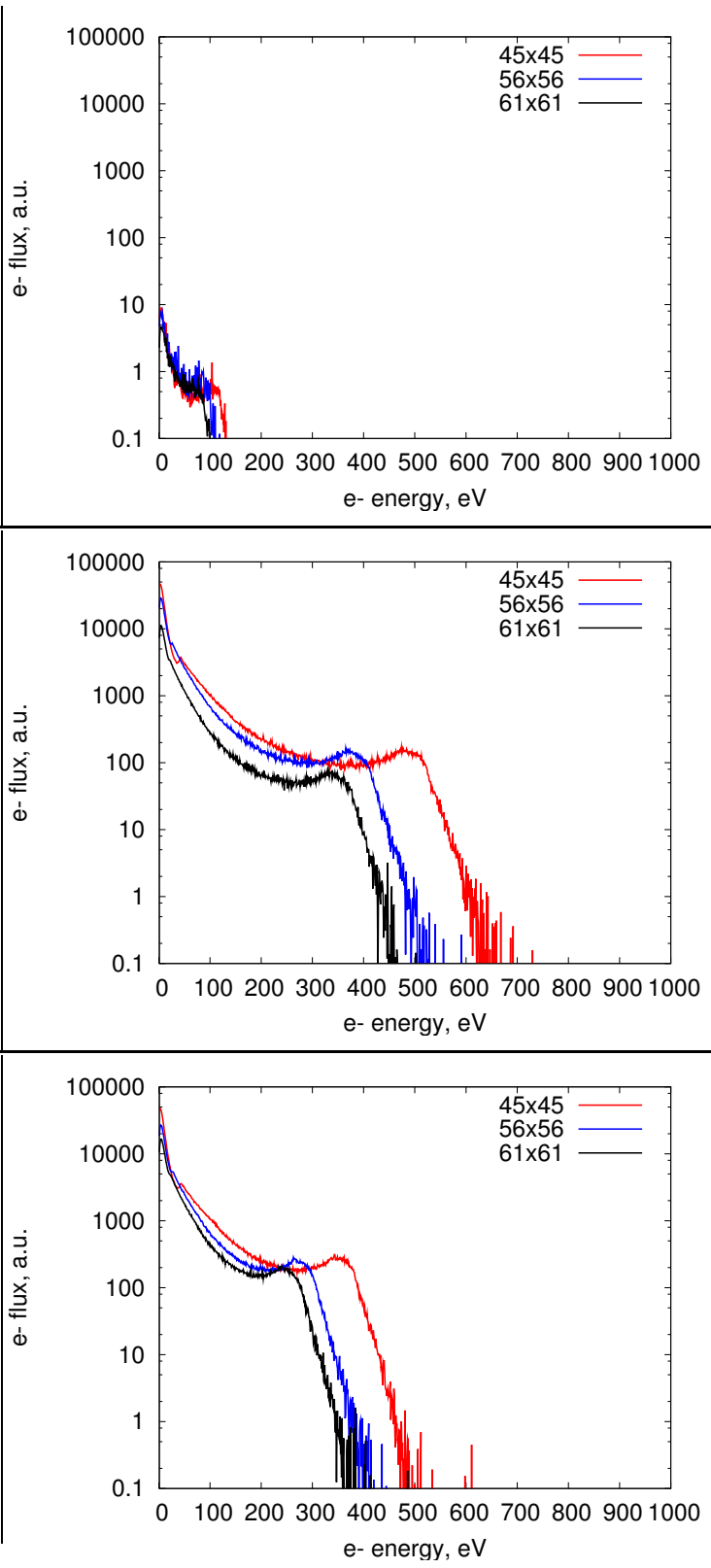

Figure 7.9: Electron cloud simulations for IR10. Depicted are the electron flux evolution, and the electron energy spectrum for bunch patterns with 45, 56, and 61 bunches per ring. 
Electron flux
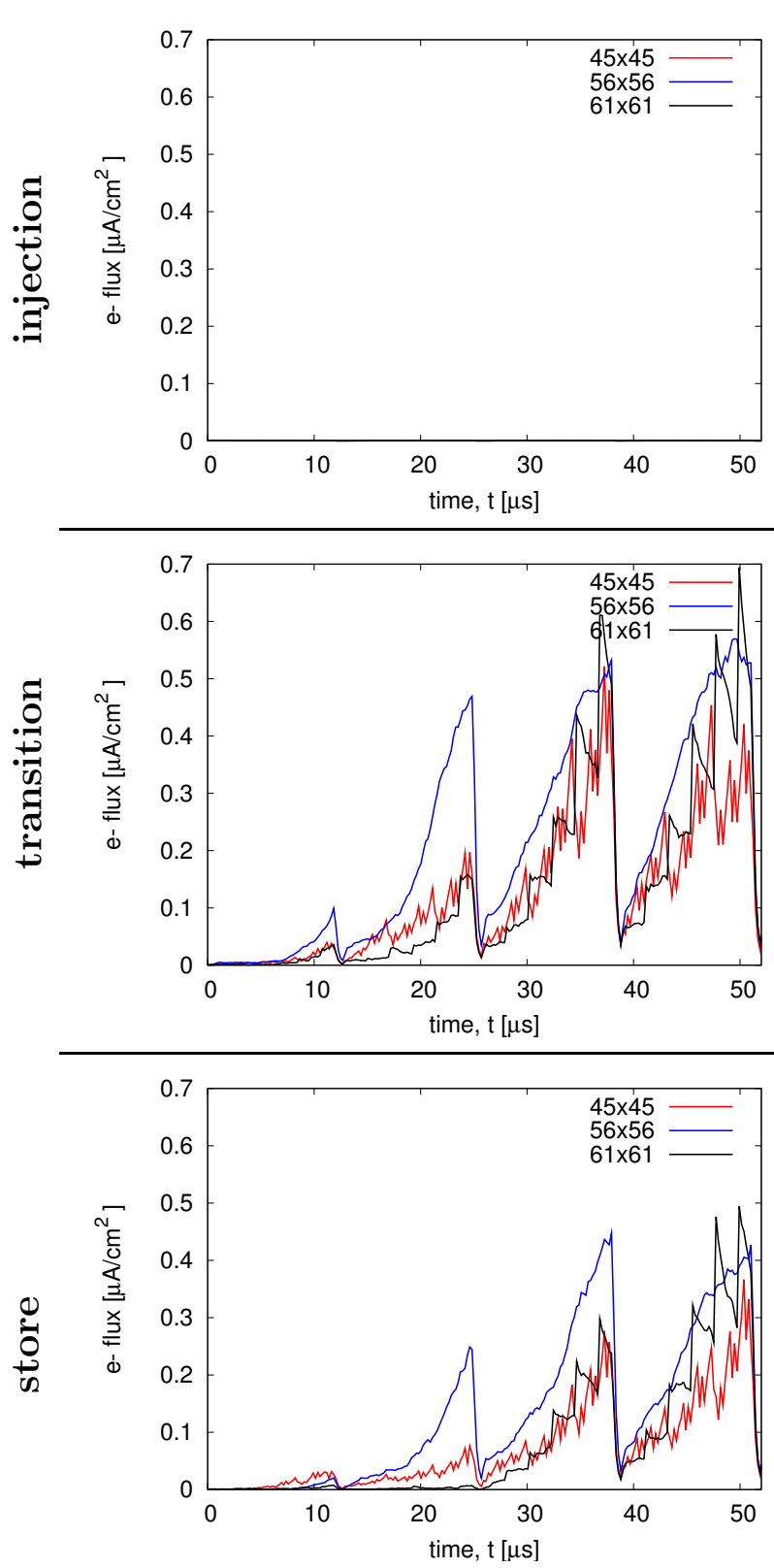

Energy spectrum
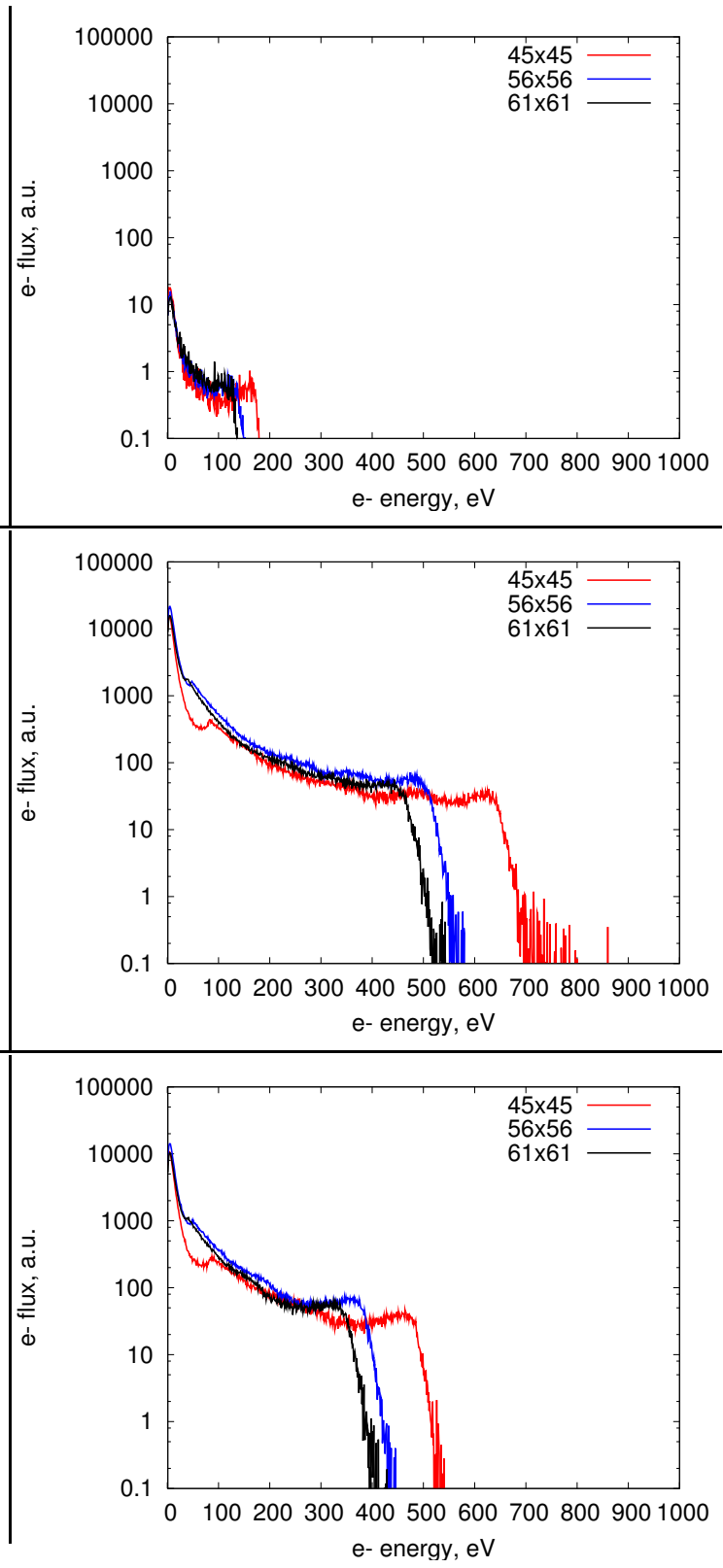

Figure 7.10: Electron cloud simulations for IR12. Depicted are the electron flux evolution, and the electron energy spectrum for bunch patterns with 45, 56, and 61 bunch. 
The intensity scaling of the transition pressure rise is within the expectations from electron clouds as the main source of the pressure rise. In the simulations the electron flux into the wall changes only slightly with the bunch pattern. A monotonic increase of the electron energy with a reduced bunch number and the same total intensity is seen. This compensates for a reduced flux into the wall and reproduces the behavior seen in the top plots in Fig. 7.8 ( $\mathbf{a}$ and $\mathbf{b}$ ), where the total beam intensity seems a relevant factor. Therefore, the conclusion is that electron clouds can explain the observed transition pressure rises.

Since the priority for an optimum collider performance is to maximize luminosity (recall Eq. 1.1), RHIC was operated with large bunch intensities and fewer bunches to reduce electron cloud effects.

\subsubsection{Beam loss induced desorption}

Figure 7.11 shows the transition pressure rise as a function of the beam loss while crossing the transition energy for IR10 (left plot) and IR12 (right plot). There appears to be no correlation between the pressure rise and the amount of beam loss. As seen in Fig. 7.7, in IR12 the pressure increase happens before any beam loss is measurable. Nonetheless, beam losses in the form of halo scraping were proposed as the main cause of the transition pressure rise [83]. For this to be true, one must assume that:

1. The halo losses are not visible in the DCCT beam (this is needed from observations in Fig. 7.7 and the middle plots at Fig. 7.8).

2. Halo losses occur in the IRs where the pressure rise is more pronounced, and

3. The desorption coefficient $\eta_{\text {loss }}$ is sufficiently large

The second point implies single-turn single-location losses. Multi-turn losses from limited dynamical aperture, tend to be concentrated in the ring locations with smallest physical aperture. Table 7.2 shows that the IRs between the DX magnets are not the limiting betatron aperture. Due to small dispersion, they are also not the limiting momentum aperture. Another single-turn loss mechanism is the electromagnetic interaction of a beam ion with the nucleus of a rest gas molecule. This is, however, not enhanced at transition and there is no reason for that process to be enhanced at a certain IR. Therefore, no loss mechanism accounting for halo scraping is seen upon transition crossing.

Table 7.2: Comparison of betatron and momentum aperture in the RHIC arcs and interaction regions.

\begin{tabular}{lcccc}
\hline \hline & $\begin{array}{c}\beta_{\mathrm{x}, \max } \\
{[\mathrm{m}]}\end{array}$ & $\begin{array}{c}\beta_{\mathrm{y}, \max } \\
{[\mathrm{m}]}\end{array}$ & $\begin{array}{c}\mathrm{D}_{\mathrm{x}, \max } \\
{[\mathrm{m}]}\end{array}$ & $\begin{array}{c}d_{\text {pipe }} \\
{[\mathrm{cm}]}\end{array}$ \\
\hline arc & 48 & 48 & 1.5 & 7 \\
DX(outside)-DX(outside) & 45 & 45 & 0.03 & 12 \\
\hline \hline
\end{tabular}



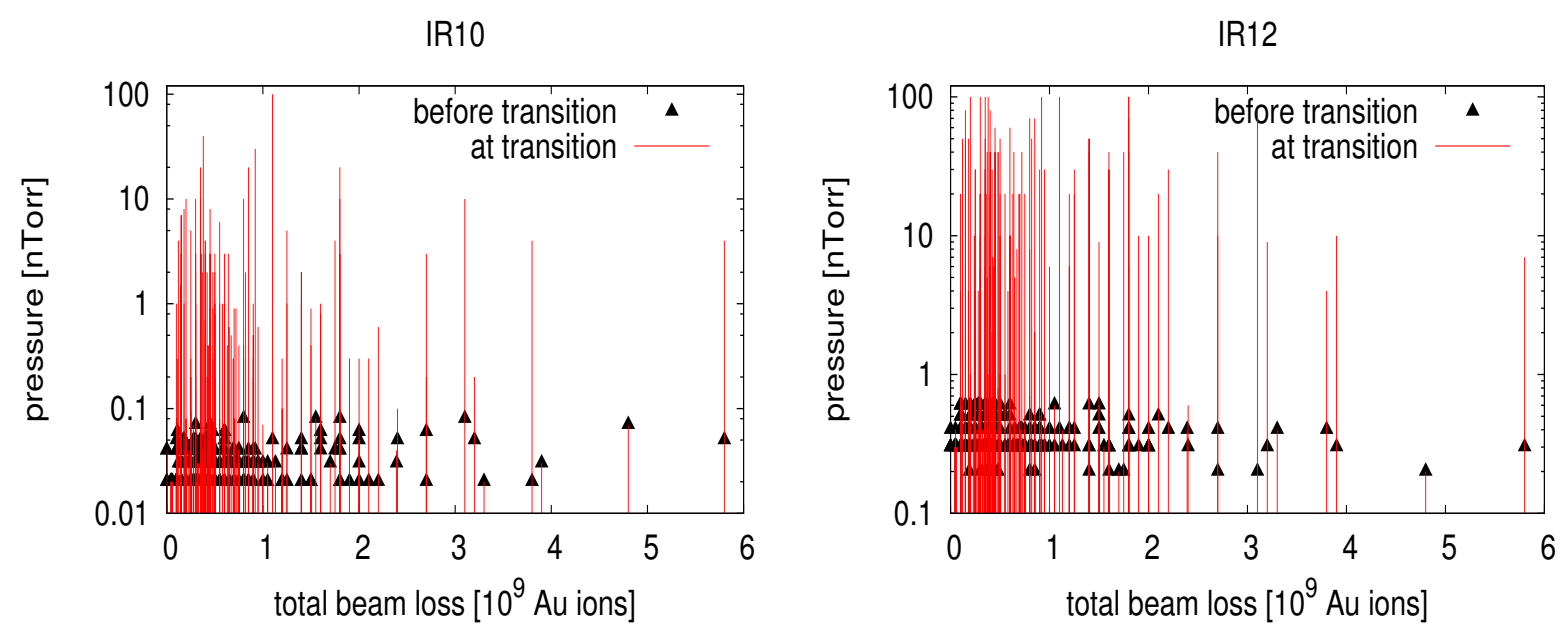

Figure 7.11: Transition pressure rise at IR10 (left) and IR12 (right), as a function of the total (blue and yellow) beam loss. The black triangles show the pressure before transition is crossed, the red vertical lines mark the pressure rise at or shortly after transition.

For the third point, a rough estimate for the required desorption coefficient $\eta_{\text {loss }}$ for beam loss driven desorption is performed. Assuming halo scraping is the main cause, the pressure rise is approximately

$$
\Delta P=\frac{k T}{2 \pi b^{2}} \frac{d N}{d l} \eta_{\text {loss }},
$$

where $k$ is the Boltzmann constant, $T$ the temperature, $b$ the beam pipe radius, and $d N / d l$ the particle loss per unit length. For a conservative estimate, assume that $10^{8} \mathrm{Au}$ ions (still visible in the DCCT) are lost within $10 \mathrm{~m}$ in IR12 leading to a pressure rise of $10^{-7}$ Torr. For this, a desorption coefficient of $\eta_{\text {loss }} \approx 10^{7}$ is needed. This is at the high end of all observations, and was only inferred from some RHIC observations [83-85]. Again, no reason is detected to enhance losses at IR12 rather than, for example, IR8.

From the above it is concluded that beam loss induced desorption cannot explain the observed transition pressure rises. 


\section{Chapter 8}

\section{Possible remedies}

Three methods are shown in this Chapter to suppress or mitigate electron cloud formation. First, it is seen that by choosing the appropriate bunch pattern, the electron density is minimized (yet the electron cloud is not fully suppressed). Second, the introduction of a longitudinal solenoid field distorts the electron motion and results in an effective mitigation of the multipacting effect. Finally, perhaps the most useful method is to decrease the SEY of the surface. In this case the beam pipe is coated with a thin layer $(\approx 1 \mu m)$ of Non Evaporable Getter (NEG), whose $\delta_{\max }$ can be as low as 1.2.

In other accelerators (see SPS case at CERN [86]), an efficient countermeasure is the so-called scrubbing effect, which consists in reducing the SEY of the chamber's material by removing the first few monolayers of its surface with electron bombardment from the multipacting process itself. Experimental data in Chapter 5 show that this measure might take a substantial amount of time, or that it requires large electron impact energies (produced by large beam bunch intensities). The latter requires that all electronics should be removed from the tunnel. These conditions could not be achieved in RHIC until 2005, and so scrubbing is not considered here. In the following, results of the three methods (bunch pattern, solenoids, and NEG) are presented.

\subsection{Bunch pattern}

Only bunch patterns with constant bunch spacing could be implemented up to 2003. Until then, RHIC operated with 55 bunches and 6 buckets spacing (214 ns), or 110 bunches with 3 buckets spacing (107 ns). In deuteron-gold operation during Run-3 (2002/2003), the pressure rise due to electron clouds at the PHOBOS experiment produced too much background, and the total number of bunches had to be reduced during beam operations from 110 to 55 in order to decrease the pressure rises (the rest of beam parameters were kept constant).

However, bunch numbers between 55 and 110 might have solved the problem as well, and would have given a larger luminosity to the experiments. For instance, for a given bunch intensity, using 68 bunches instead of 56 yields a luminosity increase of $20 \%$. How do we distribute 68 bunches to minimize the electron density? This is efficiently addressed using Maps for Electron Clouds in Chapter 9, where it is seen that the most sparse distribution of 
Chapter 8. Possible remedies

a fixed number of equal population bunches is the optimum to minimize the electron cloud density [87].

After this conclusion, the RHIC injection control system was upgraded for Run-4 (2003/2004) to implement flexible bunch patterns (i.e., bunch patterns with different bunch spacings) [88]. During gold-gold operation in Run-4, with increasing bunch intensities (from about 0.5 to about $1 \times 10^{9} \mathrm{Au}$ ions per bunch) the bunch number was gradually reduced from 68 , to 61,56 , and 45 . This allowed the PHOBOS background to be reduced while still increasing the luminosity for all experiments [78,89]. Figure 8.1 shows a snapshot of the GUI to control the injection into the RHIC ring. It shows how 68 bunches are distributed along the 120 possible buckets (with the exception of the abort gap), combining bunch spacings of 3 and 6 buckets (107 and 214 ns). Due to RHIC's 6 -fold symmetry, the bunch pattern must also have a 3-fold symmetry to provide approximately the same number of collisions to all experiments.

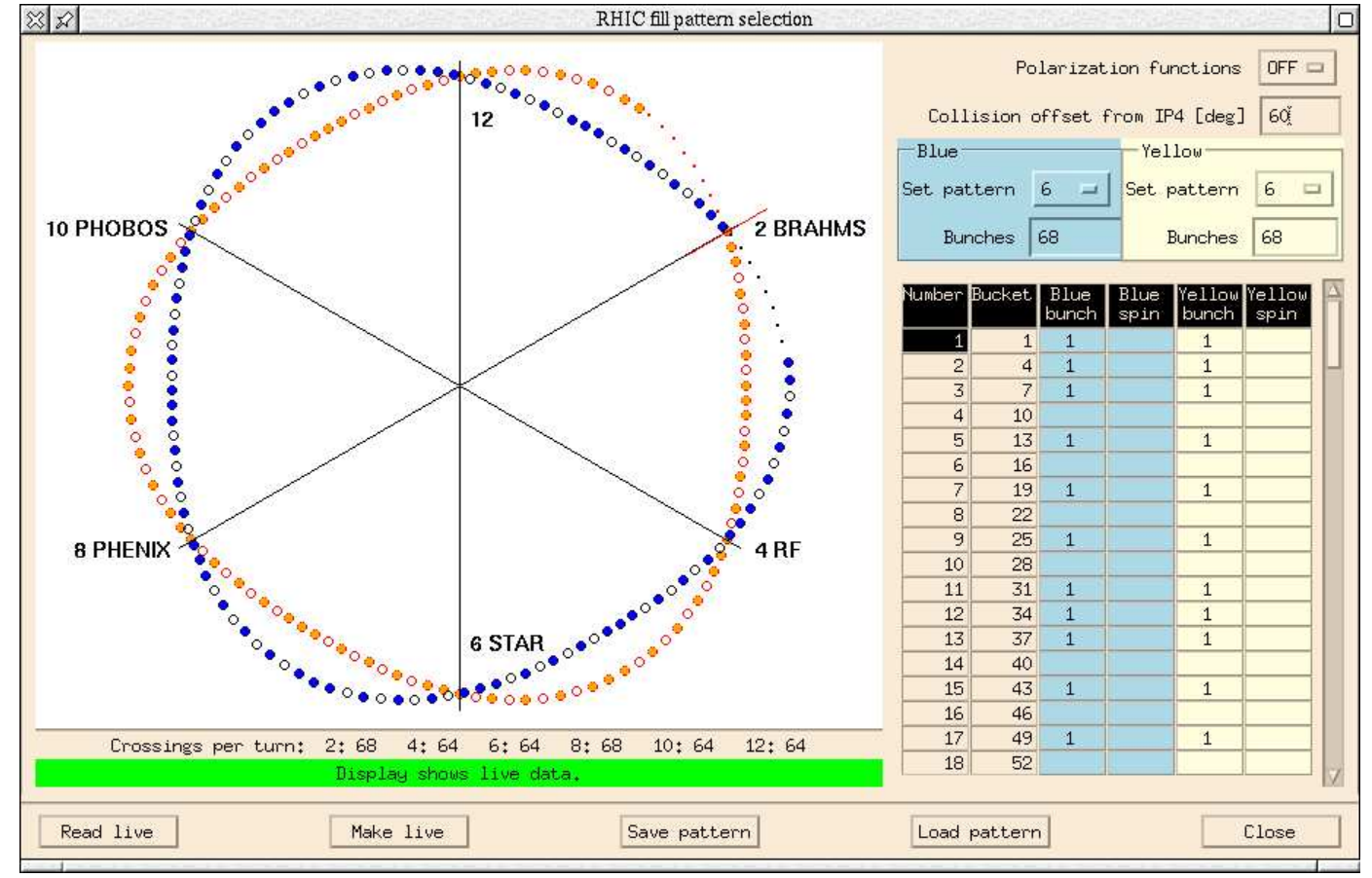

Figure 8.1: Snapshot of the GUI to control the RHIC beam injection, with the interface to set the bunch pattern [90]. Bunches can be arbitrarily placed in 110 possible locations. In this example, 68 bunches are used combining 3 and 6 buckets spacing, except for the required abort gap. Bunch 1 of the Blue beam collides with bunch 1 of the Yellow in IR2 and IR8.

\subsubsection{Operational experience using different bunch patterns}

Both the peak electron density and the average electron density are maximized if the bunches are concentrated in a single train of minimum bunch spacing, and minimized if the bunches 
are uniformly distributed around the circumference $[87,89,90]$. This will be seen in Chapter 9 , and it is not developed here to avoid redundancy.

In Chapter 7, it is seen how a reduction in the number of bunches increases the bunch intensity threshold for the transition pressure rise (see Fig. 7.8). Another experimental observation supporting the conclusion that clumping the bunches together favours an electron cloud formation is shown in Fig. 8.2. The left hand side plot in Fig. 8.2 shows the injection of 30 proton bunches in the Blue ring and 24 bunches in the Yellow ring, with 107 ns bunch spacing (3 buckets spacing). The pressure at the PHOBOS experiment in IR10 reaches $10^{-7}$ Torr. The right hand side plot in Fig. 8.2 shows the injection of 28 proton bunches in both Blue and Yellow rings with 428 ns (12 buckets spacing). The total intensity of both beams in IR10 exceeds the previous case (leftmost plot in Fig. 8.2), yet the pressure stays below $10^{-10}$ Torr. Thus, uniform bunch distributions are clearly favored to suppress electron cloud formation. This is also consistent with observations at the B-factories [90].
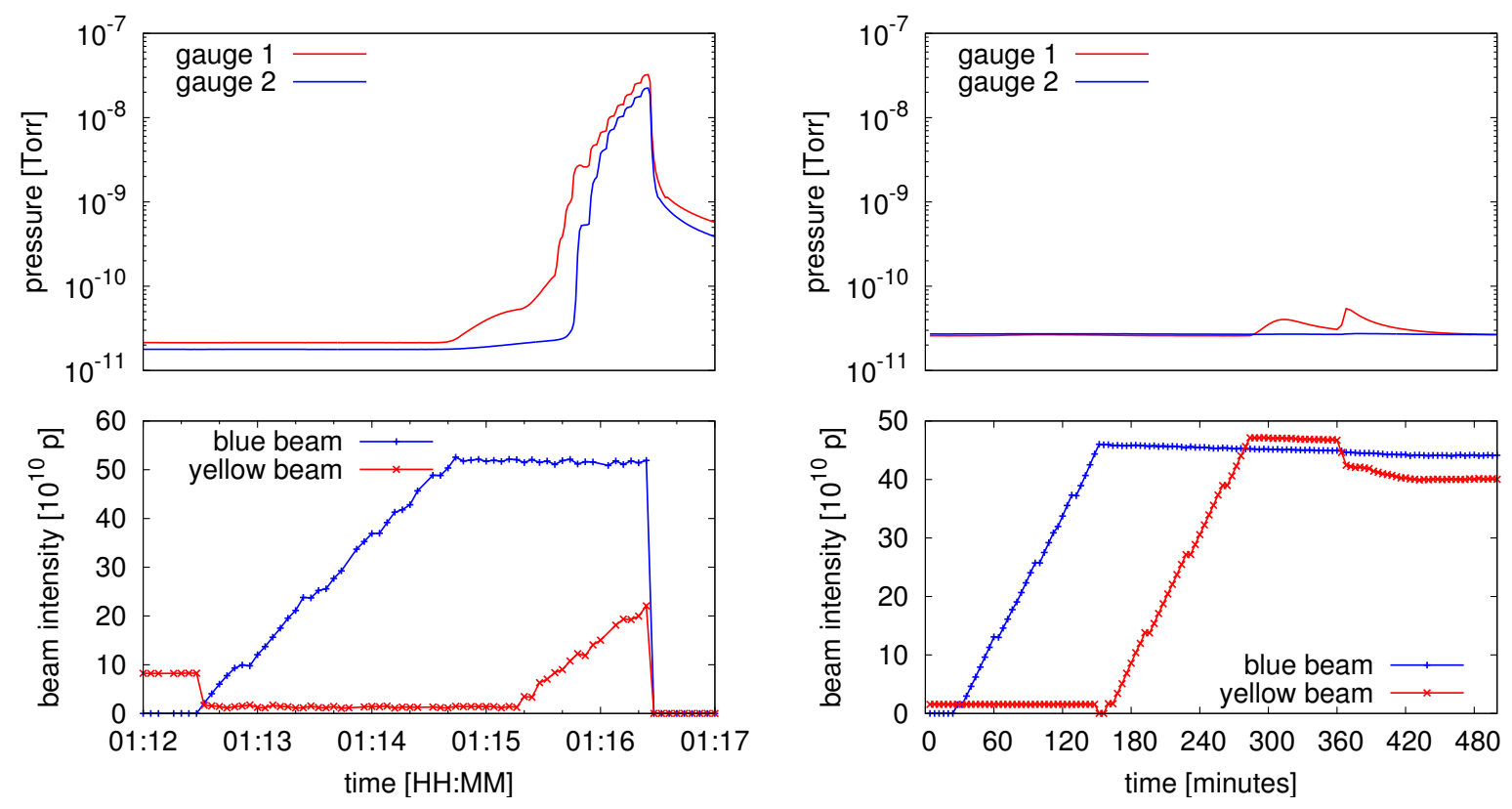

Figure 8.2: Left: Injection of 30 proton bunches in the Blue ring and 24 bunches in the Yellow ring with 3 buckets spacing. The pressure in IR10 reaches almost $10^{-7}$ Torr. Right: Injection of 28 proton bunches in both Blue and Yellow rings with 12 buckets spacing. The total intensity of both beams in IR10 is similar to the one shown in the leftmost plot, yet the pressure stays below $10^{-10}$ Torr [89].

\subsection{Solenoid fields}

In the absence of the space-charge force and in field free regions, low energy $(\sim 5 \mathrm{eV})$ electrons drift and bounce back and forth from one side of the chamber to the other. A 
weak longitudinal solenoid field forces electrons into circular orbits that hit the chamber surface after performing about half a period. Hence, the solenoid field clears the electrons between two bunch passages. In the presence of the bunch potential, electrons still perform circle-like orbits and are maintained close to the chamber's wall. Large energy gains are avoided (ideally, below $E_{1}$ in Fig. 2.1) and thus the SEY of the electron-wall collision is reduced, resulting in an effective mitigation of the multipacting effect.

Two main conditions are needed to mitigate electron clouds using solenoid fields:

1. The first condition involves the cyclotron period $T$ and the bunch spacing, $s_{b}$. A resonance occurs when half a period equals the interval between two consecutive bunches [91]

$$
T / 2=s_{b} / c
$$

where $c$ is the speed of light. For a solenoid field $B$, the cyclotron period is

$$
T=\frac{2 \pi m_{e}}{e B},
$$

where $m_{e}$ is the electron mass and $e$ is the absolute value of the electron charge. Combining Eqs. 8.1 and 8.2, the resonant magnetic field is given by

$$
B_{\mathrm{res}}=\pi \frac{m_{e} c}{s_{b} e},
$$

which amounts to 1.6 Gauss for the RHIC bunch spacing $s_{b} / c=107$ ns. If this resonance occurs, electrons hit the chamber wall in synchronism with the bunch passage and multipacting is enhanced.

2. Secondly, the Larmor radius $\rho$ of the electron trajectory has to be much smaller than the beam pipe radius, $b$. Otherwise the trajectory is not sufficiently bent, and the electron motion drifts similarly as in a field free region. This is expressed by

$$
\rho \ll b .
$$

For an electron with energy $E$, the Larmor radius is expressed by

$$
\rho=\frac{\sqrt{2 m_{e} E}}{e B} .
$$

Taking into account the electron energy spectrum shown in Fig. 3.7, two typical trajectories are expected for a given solenoid field. Assume a solenoid field of 10 Gauss: during the bunch passage, the electron energy is $E \approx 100 \mathrm{eV}$, and the Larmor radius is $\rho=3 \mathrm{~cm}$. In the interbunch, when $E \approx 8 \mathrm{eV}$, the Larmor radius is $\rho=0.6 \mathrm{~cm}$. 


\subsubsection{Electron motion in the presence of a solenoid field}

Several electron cloud simulation codes include the option of a solenoid field. At each time step, these codes compute the physical forces influencing the electron motion. The force generated by the beam's space charge potential plays an important role during a bunch passage. The transverse equations of motion in polar coordinates are [92]

$$
\begin{aligned}
& m_{e}\left(\frac{d^{2} r}{d t^{2}}-r\left(\frac{d \phi}{d t}\right)^{2}\right)=e v_{\phi} B_{z}+e E_{r} \\
& m_{e}\left(\frac{d^{2} \phi}{d t^{2}}+2 r \frac{d r}{d t} \frac{d \phi}{d t}\right)=e v_{r} B_{z} .
\end{aligned}
$$

The combination of the beam transverse electric field and the longitudinal solenoid field causes the electron to move on non trivial circular-like trajectories. In these circumstances, the Larmor radius increases because the accelerating electric field increases the electron energy. As an example, the electron cloud behaviour corresponding to the experimental conditions that will be seen in Section 8.2.2 are simulated using the ECLOUD code. The input parameters are listed in Table 8.1.
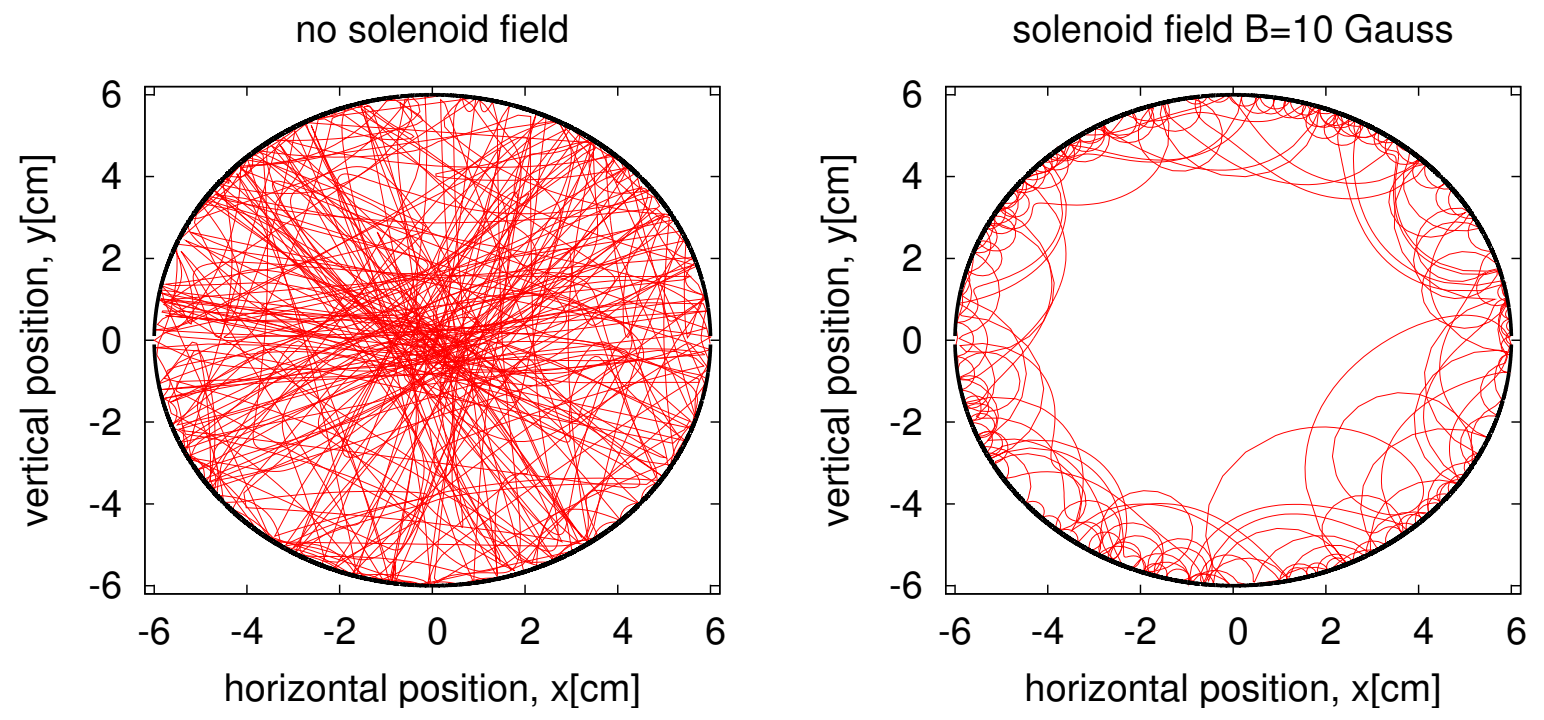

Figure 8.3: Electron trajectories in the absence (left) and in the presence of a 10 Gauss solenoid field (right).

Figure 8.3 shows the transverse $(\mathrm{x}, \mathrm{y})$ position of an electron at each time step during several bunch passages in the absence of a solenoid field (left), and in the presence of a solenoid field of $B=10$ Gauss (right). The different electron trajectories are easily recognized. In absence of the solenoid field, the electron trajectory is mainly composed by straight lines going from one side of the chamber to the other, and the electrons are almost uniformly distributed. A weak solenoid field keeps the electrons close to the chamber's wall 
(rightmost plot in Fig. 8.3). Two typical orbits are visible: first, the combination of the beam electric field and the solenoid field causes a larger Larmor radius and the electron trajectory goes close to the beam pipe axis. Second, since during the interbunch period, the solenoid field dominates, the Larmor radius is small, and the electron moves near the chamber surface with a circular-like motion.

Table 8.1: List of beam parameters during fill \#6248, when the solenoid fields were tested. The lower part of the Table shows the SEY parameters used in the ECLOUD simulation (see Ref. [47]). The charge state for $\mathrm{Cu}$ is $Z=29$.

\begin{tabular}{|l|c|c|c|}
\hline \hline parameter & symbol & unit & value \\
\hline number of bunches & $\mathrm{n}$ & $\ldots$ & 42 \\
average bunch population & $N_{b}$ & $\mathrm{Cu}$ ions & $4.5 \times 10^{9}$ \\
bunch spacing & $s_{b}$ & $\mathrm{~m}$ & 32 \\
rms bunch length & $\sigma_{z}$ & $\mathrm{~m}$ & 0.9 \\
rms bunch radius & $\sigma_{r}$ & $\mathrm{~mm}$ & 2.4 \\
revolution time & $\tau$ & $\mu s$ & 12.8 \\
beam energy & $E$ & $\mathrm{GeV} / \mathrm{n}$ & 11.2 \\
chamber radius & $b$ & $\mathrm{~mm}$ & 60 \\
solenoid field & $B$ & $\mathrm{Gauss}$ & 10 \\
\hline maximum SEY & $\delta_{\max }$ & $\ldots$ & 1.9 \\
reflection probability for $E \rightarrow 0$ & $\delta_{0}$ & $\ldots$ & 1.0 \\
energy at maximum SEY & $E_{\max }$ & $\mathrm{eV}$ & 300 \\
reflection energy & $E_{r}$ & $\mathrm{eV}$ & 60 \\
energy of secondary emitted electrons & $E_{\mathrm{sec}}$ & $\mathrm{eV}$ & 5 \\
distribution width of secondary electrons & $\sigma_{\mathrm{sec}}$ & $\mathrm{eV}$ & 4.5 \\
SEY exponent & $s$ & $\ldots$ & 1.83 \\
\hline \hline
\end{tabular}

\subsubsection{Experimental results}

For testing purposes, about $75 \mathrm{~m}$ of solenoids were installed around the RHIC circumference with a maximum field of 70 Gauss. Figure 8.4 shows an example of one of these solenoids. The inner (right) tube is the blue ring, the outer (left) is the yellow ring. This region is called BI12, and 30 out of $34 \mathrm{~m}$ in the blue ring are wrapped with a solenoid coil. This is not a uniform coil but six consecutive solenoids of about $5 \mathrm{~m}$, which are controlled by different power supplies. The bottom left part in Fig. 8.4 shows the tee with an electron detector, which is located in a gap of $\approx 0.5 \mathrm{~m}$ between two of these $5 \mathrm{~m}$ solenoids.

This setup was tested during fill \#6248 in Run 5 (2004/2005), whose characteristics are listed in Table 8.1. The evolution of the peak electron signal and the pressure along the BI12 chamber is depicted in Fig. 8.5. Both the pressure and the peak electron signal in the detector increases sharply after 28 bunches are injected, and the electron cloud builds up (at about 13:46). Next, the six solenoids are powered to 13.5 Gauss (at 13:47), and consequently the pressure decays from 42 to 8 nTorr and the electron signal decreases by a 
factor of about 3. Note that a later increase in the solenoid field to 27 Gauss reduces neither the pressure nor the electron signal, compared to previous levels. This points towards a saturation of the solenoid effect. At 13:49:30, the acceleration ramp starts and the bunches become shorter, producing a peak in the electron and pressure signals up to the transition crossing (13:50:10). At this point, the beam loses about half of its intensity and the electron cloud is not sustained anymore.

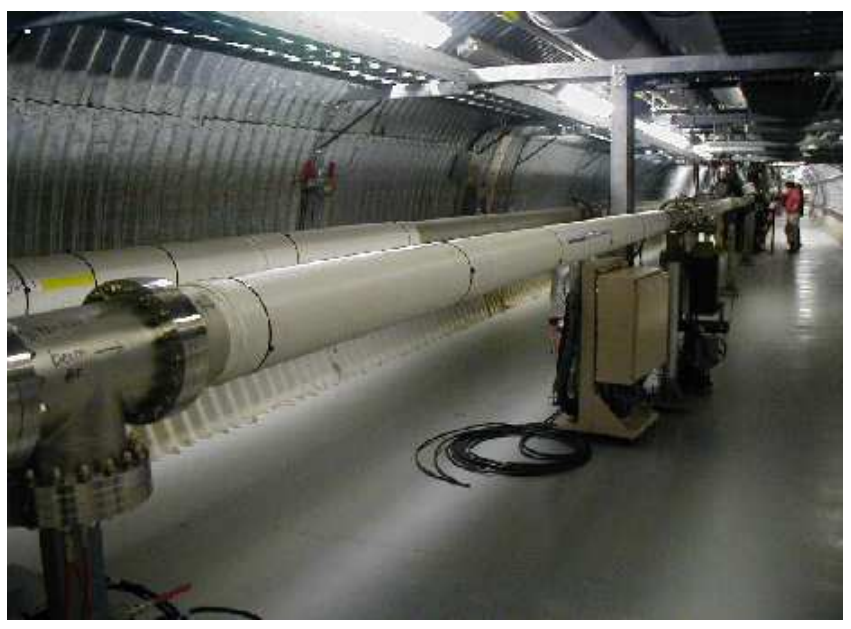

Figure 8.4: The picture shows an example of a solenoid at BI12, where 30 out of $34 \mathrm{~m}$ are wrapped with solenoids.

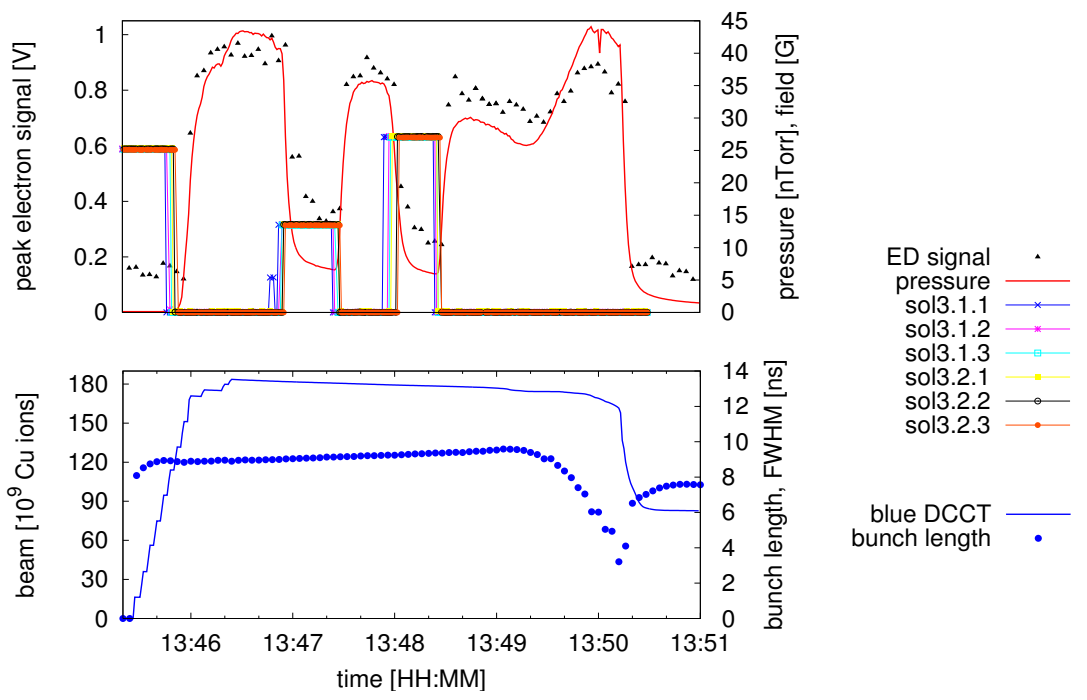

Figure 8.5: Effect of the solenoid field at BI12. Both the pressure and the electron detector signal decrease to a similar level for a magnetic field of 13.5 Gauss and 27 Gauss. At about 13:49:30, the acceleration ramp starts, the bunch length is reduced, and pressure and electron signals increase. Half of the beam is lost while crossing the transition energy (13:50:05), after which the electron cloud disappears because of the low bunch intensity. 
Note that both the pressure and the electron signals show a residual effect when the solenoid field is applied. This might be due to the non ideal solenoid field created in the inter-solenoid gaps.

\subsection{Non Evaporable Getter coatings}

\subsubsection{Motivation for a getter film}

One way to reduce the electron cloud is to decrease the SEY of the chamber wall. Several methods have been tried. For instance, baking a stainless steel chamber reduces the maximum SEY from $\delta_{\max } \approx 2.1$ [46] to about $\delta_{\max } \approx 1.8$ [93]. Baking not only reduces the SEY, but also the electron induced molecular desorption coefficient $\eta_{e}$. Unfortunately, electron clouds are still created in baked stainless steel surfaces (see Fig. 6.3 for a difference between a baked and an unbaked surface).

Lower SEYs than those characteristic of baked surfaces are achieved by coating the vacuum chamber with a thin film of Non Evaporable Getter (NEG). A getter is a material widely used to achieve Ultra High Vacuum pressures. It sorbs gas molecules on the surface in the form of stable chemical compounds. These materials are generally alloys made of Titanium (Ti), Barium (Ba) or Zirconium (Zr). There are typically two ways to initiate the chemical process to trap the molecules into the getter bulk:

1. by "in situ" deposition of a fresh getter film, called an evaporable getters. The best known example is a Titanium Sublimation Pump, in which a Ti filament is heated up to about $1500^{\circ}$, a temperature at which the Ti vapour pressure is about $10^{-3}$ Torr. Titanium then provides large sticking probabilities for the components present in the accelerator vacuum chamber $\left(H_{2}, C O\right.$, etc $)$.

2. by heating an oxidized getter to a temperature high enough to diffuse oxygen from the surface into the getter bulk. This is called a Non Evaporable Getter (NEG). The required heating temperature is called activation temperature, and it is typically around $200^{\circ} \mathrm{C}$.

Coating the inner surface of the beam pipe wall with a thin film (around $1 \mu \mathrm{m}$ ) of NEG transforms the vacuum chamber from a gas source to a pump. And, most important for electron clouds, an activated NEG decreases the chamber SEY. Activation is carried out by heating up the NEG material and promoting the diffusion of oxygen of the passivating surface layer until the surface is sufficiently clean to start sorbing the impingin gases - see Fig. 8.6. If the NEG material is exposed to large enough $\mathrm{CO}$ or $\mathrm{CO}_{2}$ partial pressures, the NEG becomes saturated or contaminated. That is to say, one monolayer of $\mathrm{CO}$ or $\mathrm{CO}_{2}$ is formed on the getter surface and the chemical reactivity (hence, the pumping effectivity) is drastically reduced. More information about getters can be found in Refs. [93-95]. The influence of these processes on the SEY of a NEG material deposited on a stainless steel surface is shown in Fig. 8.7, where the SEY is plotted as a function of the impinging electron energy. Note that $\delta_{\max }$ is reduced from about 2.0 ("as received" NEG) to about 1.3 after 
activation. Recontamination increases its SEY, but it is still smaller than an "as received" NEG (top plots in Fig. 8.7). By re-activating the NEG, $\delta_{\max }$ is reduced again to about 1.3 .
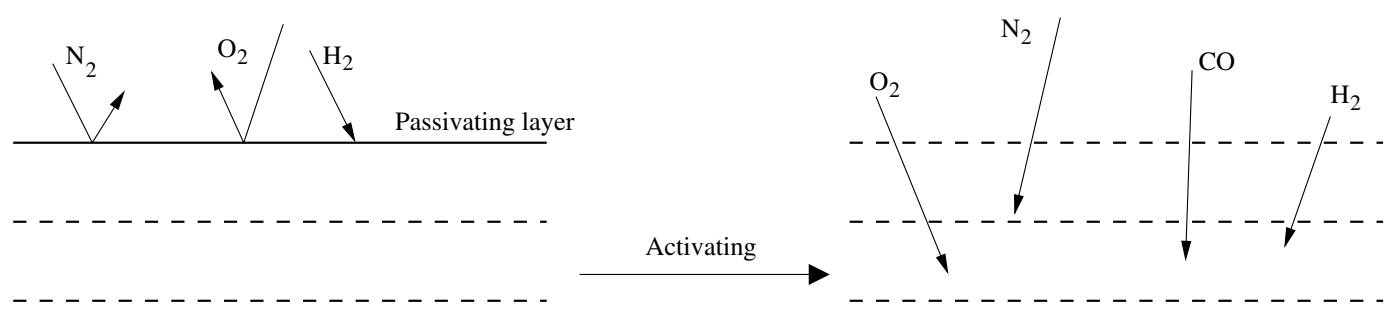

Inactive TiZrV NEG

Active TiZrV NEG

Figure 8.6: Activation of the TiZrV getter film [21]: heating up the material removes the passivating layer so that the molecules impinging the NEG are trapped in the bulk material.

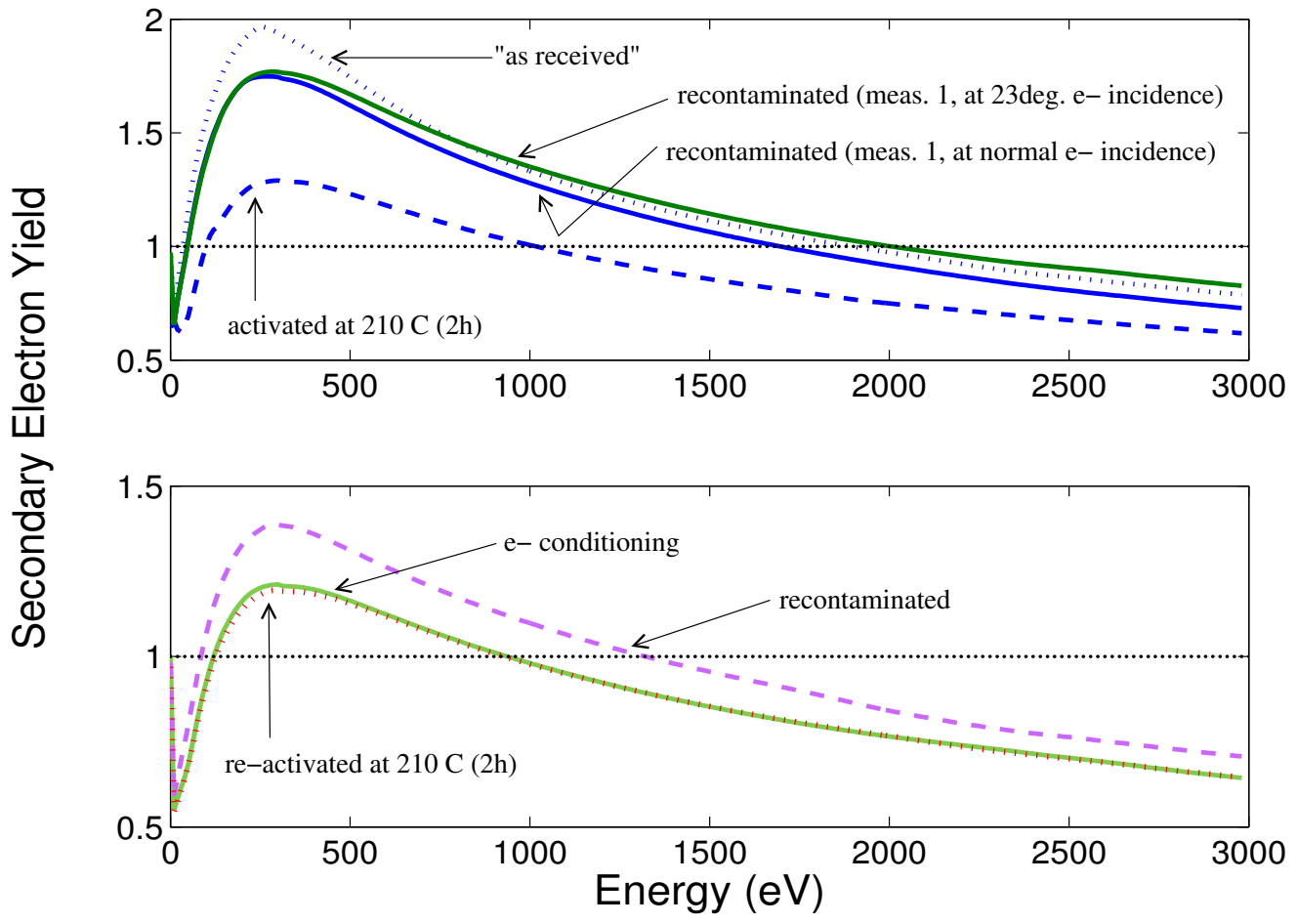

Figure 8.7: SEY of the TiZrV getter film on a stainless steel surface [93]. The top plot shows NEG "as received" (dashed upper line), activated at $210^{\circ} \mathrm{C}$ during $2 \mathrm{~h}$ (dashed line with lower $\delta_{\max }$ ), and vacum recontaminated (middle curves with solid lines). The bottom plot shows the electron conditioning (solid line), vacuum recontaminated after 34 days (dashed line), and re-activated by re-heating the material at $210^{\circ} \mathrm{C}$ during $2 \mathrm{~h}$ (dotted line). Data courtesy of F. Le Pimpec. 


\subsubsection{Evaluation of the NEG coating installed at RHIC}

The NEG coating on the chambers installed at RHIC is an alloy made of Titanium, Zirconium, and Vanadium, TiZrV [95]. The main reasons for this choice of alloy are the low maximum SEY after activation and the low activation temperature $\left(\approx 200^{\circ} \mathrm{C}[95]\right)$. The maximum SEY after activation is expected to be $\delta_{\max } \approx 1.3$, remaining low even after recontamination of the surface (see Fig. 8.7).

Around $200 \mathrm{~m}$ of NEG coating was performed during the summer 2004 by magnetron sputtering at SAES Getter in Milan [96,97]. The coated (but not yet activated!) chambers were then shipped to BNL, and they were finally installed around the RHIC's warm bore sectors by Run 5 (2004/2005). The activation process was carried out during the standard baking process, providing a linear pumping. Static pressures below $10^{-11}$ Torr are expected in the RHIC vacuum pipe, although the pressure gauges cannot measure such low values [98].

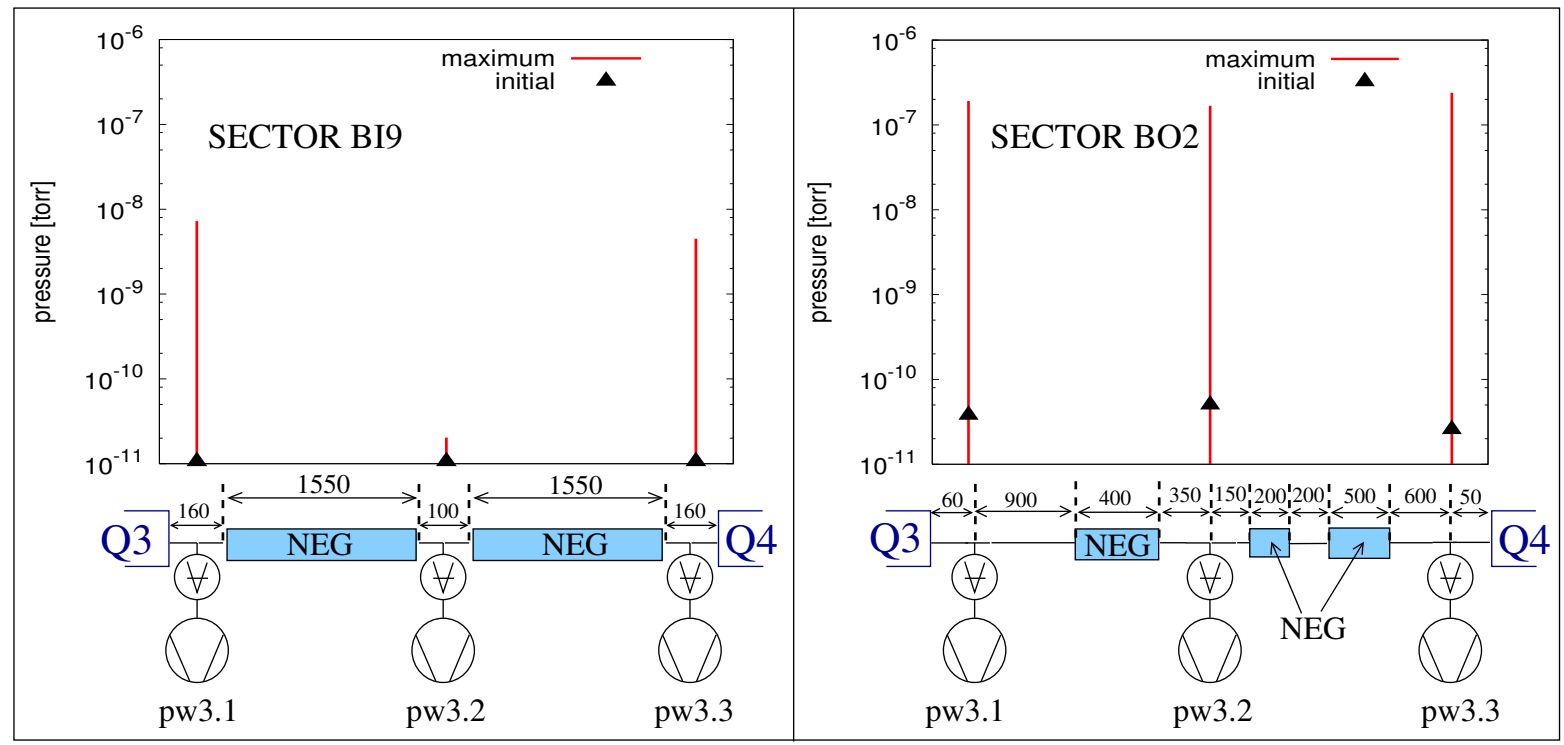

Figure 8.8: Effect of the NEG coated beam pipes between the magnets Q3 and Q4 (the two cold bores) in the blue warm bores at sector 9 (left) and 2 (right).

In case of electron clouds, the final pressure at a given gauge depends on the fraction of the beam pipe coated with NEG, and the gauge position relative to the NEG coating. This was tested during fill \#6248 (characteristics listed in Table 8.1 and shown in Fig. 8.5 bottom plot). The effectiveness of the NEG coating is shown in Fig. 8.8, which depicts the static (black triangles) and maximum pressure (red lines) during fill \#6248 for sector BI9 (left), and BO2 (right). The bottom plot shows a sketch of the vacuum equipment at each location: NEG coating, vacuum pumps and gauges. For testing purposes, the vacuum pump at port "pw3.2" in sector BI9 was switched off. At BI9, the static pressure at all gauges is at the lower limit of the gauge detection $\left(10^{-11}\right.$ Torr $)$, and it barely increases in the middle 
gauge ("pw3.2") during all the fill. However, at both edges (gauges "pw3.1" and "pw3.3") the pressure increases to about $5 \times 10^{-9}$ Torr. The reasons for these pressure rises are uncertain, but it is believed they are due to the electron cloud produced within the $160 \mathrm{~cm}$ of stainless steel where the gauges are located. Gas diffusion from the cold bore is also possible. In the middle gauge, although electron clouds might be produced in the $100 \mathrm{~cm}$ of uncoated stainless steel, the large amount of NEG coating close to this location compensates the outgassing due to the multipacting even though the vacuum pump at "pw3.2" is switched off.

By contrast with BI9, the pressure at all three gauges in BO2 increases to about $2 \times 10^{-7}$ Torr (two orders of magnitude larger than in BI9), even though there are $11 \mathrm{~m}$ of NEG coated beam pipes. In this case gas desorption from electron clouds is (likely to be) produced in the $\sim 23 \mathrm{~m}$ of the uncoated stainless steel. Molecules rapidly diffuse along the beam pipe and the NEG capacity is not enough to suppress the pressure rise. Thus, due attention should be paid to the distribution of the NEG coated pipes along a real machine section. Unfortunately, BO2 is a region with many beam instruments and so a complete coating (as in BI9) is difficult.

\subsection{Future strategies}

In the the near future, it is not planned to install solenoids on a large scale in RHIC. Solenoids are as expensive as the NEG coating, and NEG efficiency is more reliable. Moreover, powering the solenoids slightly heats the vacuum chamber, which produces a thermal outgassing that results in a larger static pressure. NEG coating is especially suitable for the interaction regions: while solenoids reduce the transparency of the experiments because of the Copper wires wrapped around the chamber, the $\sim 1 \mu \mathrm{m}$ NEG layer does not affect the chamber transparency, decreases the vacuum pressures, and hence reduces the experimental background. Meanwhile, the bunch pattern is always optimally distributed to mitigate the detrimental effects of the unavoidable electron clouds. 



\section{PART III}

MAPS FOR ELECTRON CLOUDS 



\section{Chapter 9}

\section{Maps for electron clouds}

The electron cloud effect has been studied by means of detailed simulation codes that typically track the particles evolution under the influence of the corresponding electromagnetic forces and fields. In this Chapter it is shown that, for the RHIC case, the electron cloud can be treated from an abstract point of view as a bunch to bunch evolution using simple maps. Next, it is shown how this treatment yields a useful conclusion, which is otherwise difficult to obtain: for a fixed number of bunches and total beam current in RHIC, it is possible to determine the best way to distribute the bunch pattern around the ring to minimize the electron cloud formation.

\subsection{Motivation}

Several computer simulation codes were (and still are being) developed and compared with experimental observations to study the electron cloud effect. A pioneer model is found in Ref. [99], while a comparison among the different codes can be seen at Ref. [54]. Typically, an electron cloud code work either by Particle In Cell methods (like CLOUDLAND), or by tracking electrons grouped into macro-particles, where each macro-particle comprises up to a $10^{5}$ electrons (like ECLOUD, or CSEC). When a macro-particle produces more electrons, its total Coulomb charge is increased. At every time step, these detailed codes compute the necessary physical forces and fields influencing the motion of the macro-particles. If electron cloud formation takes place, the codes track about $10^{10}$ electrons per meter of beam pipe (depending on the parameters). Hence, these codes use a large amount of CPU time: a complete electron cloud simulation, depending on the input code parameters, can last from around 1 hour to some days. In the cases studied here (for the parameters in Table 9.1), a single simulation lasts about 1 hour.

In the following, it is considered that for given beam pipe characteristics (SEY, chamber dimensions, et cetera), the electron density after bunch $m$ passes by (referred to as $\rho_{m+1}$ ), is a function only of the interaction between the bunch and the electron density before bunch $m$ passed by (referred to as $\rho_{m}$ ). This is expressed by means of an iterative formalism. For 
instance, in a parabolic map:

$$
\rho_{m+1}=a \rho_{m}+b \rho_{m}^{2},
$$

where the parameters $a$ and $b$ are functions of beam parameters such as bunch intensity, $N$; bunch spacing, $s_{b}$; rms bunch length, $\sigma_{z}$; and rms bunch transverse size, $\sigma_{t}$. Ultimately, $a$ and $b$ are functions of the beam pipe characteristics as well: maximum SEY, $\delta_{\max }$; electron energy at which SEY is maximum, $E_{\max }$; reflectivity at zero electron energy, $\delta_{0}$; beam pipe dimensions, et cetera. Therefore, the coefficients $a$ and $b$ summarize the electron cloud dependence on the physical parameters. This parabolic map is equivalent to the "logistic" difference equation [100], since by introducing the dimensionless variable $X \equiv b \rho / a$, and for $a>0, b<0$, Eq. 9.1 can be expressed as:

$$
X_{m+1}=a X_{m}\left(1-X_{m}\right),
$$

which reproduces the logistic map formalism [100] with all its richness. For small $\rho$, Eq. 9.1 reflects the exponential growth with the bunch passage [67],

$$
\rho_{m} \approx \rho_{0} e^{(a-1) m},
$$

where it is clear that electron cloud density grows for $a>1$, and otherwise the cloud collapses. Eventually, this unlimited growth is stopped by the space charge effects created by the electrons themselves.

From Eq. 9.1 in a parabolic mode, the saturated electron cloud density, $\rho_{\text {sat }}$ is determined as a function of the bunch intensity $N$ simply by:

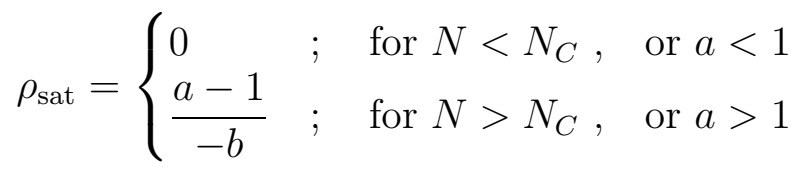

where $N_{C}$ marks the bunch intensity threshold for the electron cloud. Equation 9.4 shows a phase transition from electron cloud "off" to "on". If $a$ and $b$ increase smoothly with $N$, the phase transition is second order. However, RHIC data show both first and second order electron cloud phase transitions [79]. This is not yet well understood, and is further addressed in Chapter 11. The parabolic model of Eq. 9.1 is a mathematical tool illustrating the goal in this Chapter: to simplify the electron cloud problem by using only a small number of mathematical parameters. In this example, these parameters are $a$ and $b$.

If the electron cloud evolution can be described using a simple map $\rho_{m+1}=f\left(\rho_{m}\right)$, this frees up the detailed simulation codes and enhances physical intuition through the use of simple mathematics. Next, an evaluation of whether it is possible to follow the electron density in a bunch to bunch evolution is performed (Section 9.2). Second, it is desirable to obtain a suitable function to follow this evolution (Section 9.3). Finally, one application of map modeling is presented (Section 9.4). 


\subsection{The bunch to bunch evolution}

A typical time evolution of the electron density is shown in Fig. 9.1. This evolution corresponds to a CSEC simulation where 60 bunches of $1.4 \times 10^{11}$ protons each, spaced $107 \mathrm{~ns}$ apart, are injected into the RHIC ring. The red line shows CSEC output, while the gray circles mark the average electron density between the passage of two bunches. The presence of a bunch is indicated by the gray bars at the bottom of the figure, the light blue bars mark an empty bunch. The electron density per beam pipe meter as a function of time, $\rho$ grows exponentially until the space charge effects produce a saturation level (see Section 3.4 and References therein). Once the saturation level is reached the average electron density does not change significantly. In the bunch to bunch evolution, the time step is one bunch passage. Figure 9.1 shows that sampling the evolution on a bunch-to-bunch' basis is sufficient for retaining information about the build-up and decay times, although the details of the behaviour of the electron density oscillation between two bunches is lost.

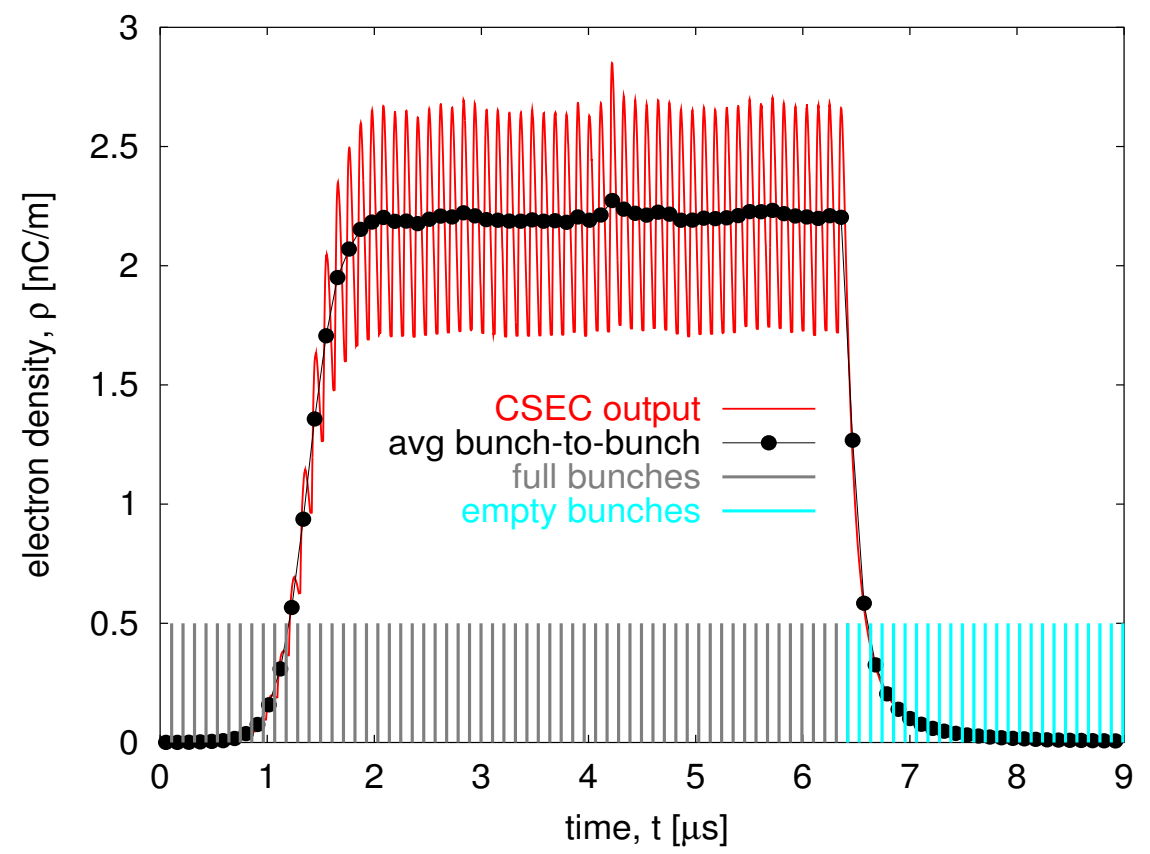

Figure 9.1: Time evolution of the electron density (red line) computed with CSEC during $9 \mu$ s. The RHIC revolution period is $12.8 \mu \mathrm{s}$. This case corresponds to the injection of 60 successive bunches with a bunch spacing of $107 \mathrm{~ns}$ and a bunch intensity of $N=1.4 \times 10^{11}$ protons (marked with grey bars), followed by 60 "empty" bunches (marked with light blue bars). The grey circles mark the average electron density between two consecutive bunches.

Do existing computer simulations confirm that the electron cloud evolution can be represented by maps? Two codes are used to test this hypothesis: CSEC (described in Chapter 3) and ECLOUD [101], focusing the studies on the RHIC case. Table 9.1 shows the physical parameters used for these simulations. All simulation codes depend strongly 
Table 9.1: Input parameters for electron cloud simulations testing the map hypothesis. In all cases, the simulations using CSEC and ECLOUD are performed for protons bunches.

\begin{tabular}{lcccc}
\hline \hline & & & CSEC & ECLOUD \\
\hline parameter & symbol & unit & value & value \\
\hline bunch spacing & $s_{b}$ & $\mathrm{~ns}$ & 107 & 107 \\
number of bunches & $M$ & $\ldots$ & 60 & 60 \\
rms bunch radius & $\sigma_{t}$ & $\mathrm{~mm}$ & 2.4 & 2.4 \\
full bunch length & $\sigma_{z}$ & $\mathrm{~ns}$ & 18 & 21 \\
protons per bunch & $N$ & $10^{10}$ & 8 to 20 & 8 to 20 \\
revolution time & $t_{\mathrm{rev}}$ & $\mu s$ & 12.8 & 12.8 \\
beam energy & $E$ & $\mathrm{GeV}$ & 27.7 & 11.46 \\
\hline beam pipe diameter & $d$ & $\mathrm{~mm}$ & 120 & 120 \\
reflectivity at zero energy & $\delta_{0}$ & $\ldots$ & 0.6 & 1.0 \\
reflectivity at infinite energy & $\delta_{\infty}$ & $\ldots$ & 0.2 & $\ldots$ \\
rediffusion probability & $P_{\mathrm{rd}}$ & $\ldots$ & 0.5 & $\ldots$ \\
reflection energy & $E_{\mathrm{rf}}$ & $\mathrm{eV}$ & 60 & 60 \\
maximum SEY & $\delta_{\max }$ & $\ldots$ & 2.3 & 2.3 \\
energy for maximum SEY & $E_{\max }$ & $\mathrm{eV}$ & 310 & 310 \\
energy for secondary electron & $E_{\mathrm{sec}}$ & $\mathrm{eV}$ & 8.9 & 7.0 \\
energy width for secondary electron & $\sigma_{\mathrm{sec}}$ & $\mathrm{eV}$ & 4.5 & 5.5 \\
\hline initial e- density & $\rho_{\mathrm{ce}}$ & $\mathrm{pC} / \mathrm{m}$ & 0.2 & - \\
electrons generated per bunch & $\ldots$ & $\ldots$ & 35000 & - \\
electron generation radius & $\ldots$ & $\mathrm{mm}$ & 60 & - \\
\hline number of slices per bunch & $\ldots$ & $\ldots$ & 60 & 100 \\
number of slices per inter-bunch & $\ldots$ & $\ldots$ & 840 & 100 \\
initial number of macro-particles & $\ldots$ & $\ldots$ & 25 & - \\
maximum number of macro-particles & $\ldots$ & $\ldots$ & $10^{5}$ & $\approx 10^{5}$ \\
\hline \hline
\end{tabular}

on the model used for the SEY behavior [47]. CSEC uses the model described in Ref. [46], while ECLOUD uses the model described in Ref. [47].

With 107 ns bunch spacing and the RHIC revolution period, one can inject up to 120 bunches (not counting the limitations given by the abort gap kickers, which decrease this number to 110). For the purpose of this study we are interested in the build up and decay of the electron density. Therefore and to minimize CPU time, simulations only need to be performed with a bunch train of 60 consecutive bunches (until saturation is reached).

The bunch to bunch evolution of the electron cloud density is followed for different bunch intensities, $N$, ranging from $8 \times 10^{10}$ to $2 \times 10^{11}$ protons, in steps of $\Delta N=2 \times 10^{10}$ protons using the parameters listed in Table 9.1. Figure 9.2 shows how the electron density after bunch $m$ passes by, $\rho_{m+1}$, behaves as a function of the previous electron density, $\rho_{m}$, for different bunch intensities, $N$. The points in Fig. 9.2 show the average electron cloud density between two bunches using results from CSEC (Fig. 9.2, left) and ECLOUD (Fig. 9.2, right). The lines correspond to cubic fits with no constant term (see below). 
Figure 9.2 is explained as follows: starting with a small seed of electrons, electron density $\rho_{0} \approx 0 \mathrm{nC} / \mathrm{m}$, the density grows and reaches the saturation line $\left(\rho_{m+1}=\rho_{m}\right.$, red trace) when the space charge effects due to the electrons of the cloud itself limit further growth. In this situation, all the points (corresponding to the passage of full bunches) are in the same spot on the $45^{\circ}$ line. This particular line, showing $\rho_{m+1}=\rho_{m}$, is also called identity map [100].

CSEC

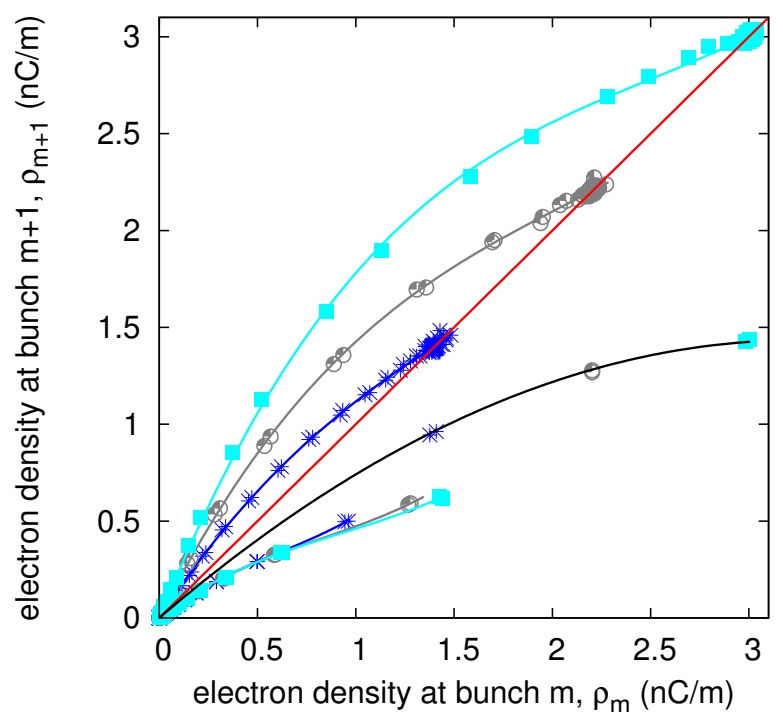

ECLOUD

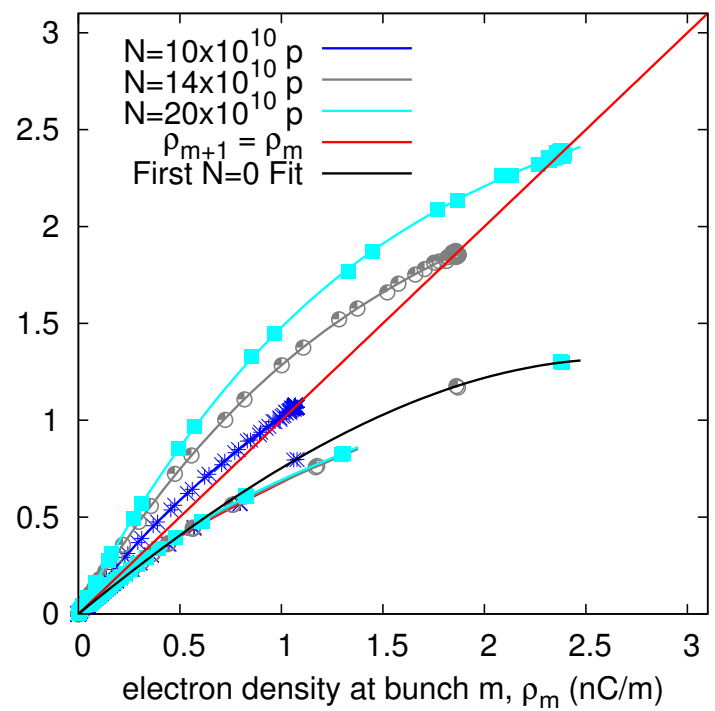

Figure 9.2: Average electron density after bunch passage $m, \rho_{m+1}$, as a function of the electron density before bunch $m$ passed by, $\rho_{m}$, for different bunch intensities, $N$. The left plot shows the CSEC output, while the points on the right hand side come from ECLOUD simulation. In both cases, the lines correspond to cubic fits applied to the average bunch to bunch points.

Electron cloud decay occurs during a succession of bunches with null intensity, $N=0$. Except for the point corresponding to the electron cloud density after the first empty bunch, the electron density follows a universal curve independent of the initial value of the saturated electron density. The "first empty bunch" points after the identity map (from different saturation values, $\rho_{\text {sat }}$ ) lie off the universal curve on the "first $N=0$ ", or "first empty bunch" curve. This is arguably related to the space charge effects during saturation and the energy of the cloud electrons: the energy of the cloud electrons is due to the bunch kick, and this energy should be strictly zero for bunches with null intensity $(N=0)$. However, after an electron cloud formation, the electrons energy is not null because of the reminiscences of the previous non-null bunch interaction. For the CSEC case, the difference is larger than for the ECLOUD case. A possible explanation is that ECLOUD does not take into account the "rediffused" electrons (see Section 3.3): the secondary electrons are either "reflected" or "true secondaries". Thus, the cloud energy decreases more rapidly for ECLOUD than CSEC. Despite the difference between the two codes, data in Fig. 9.2 shows it takes two bunches to jump from a curve $N \neq 0$ to the decay ( $N=0$ curve). 


\subsection{The map candidates}

Different candidate forms for the parametric maps include the abovementioned "parabolic" map (Eq. 9.1), the "cubic" map (with no independent term),

$$
\rho_{m+1}=a \rho_{m}+b \rho_{m}^{2}+c \rho_{m}^{3}
$$

and an "asymptotic" map,

$$
\rho_{m+1}=\frac{a \rho_{m}}{1+b \rho_{m}}
$$

which is also known as "Hassell" model in density-dependent population dynamics $[102,103]$.

Figure 9.3 shows the results for the $\chi^{2}$ coefficient for each fit to the data in Fig. 9.2, and for each bunch intensity, $N$, for both CSEC (left plot) and ECLOUD (right). Since the smallest $\chi^{2}$ value corresponds to the cubic map, we continue the analysis using cubic maps, stating clearly that this map is valid only for electron densitites within the ranges used here.
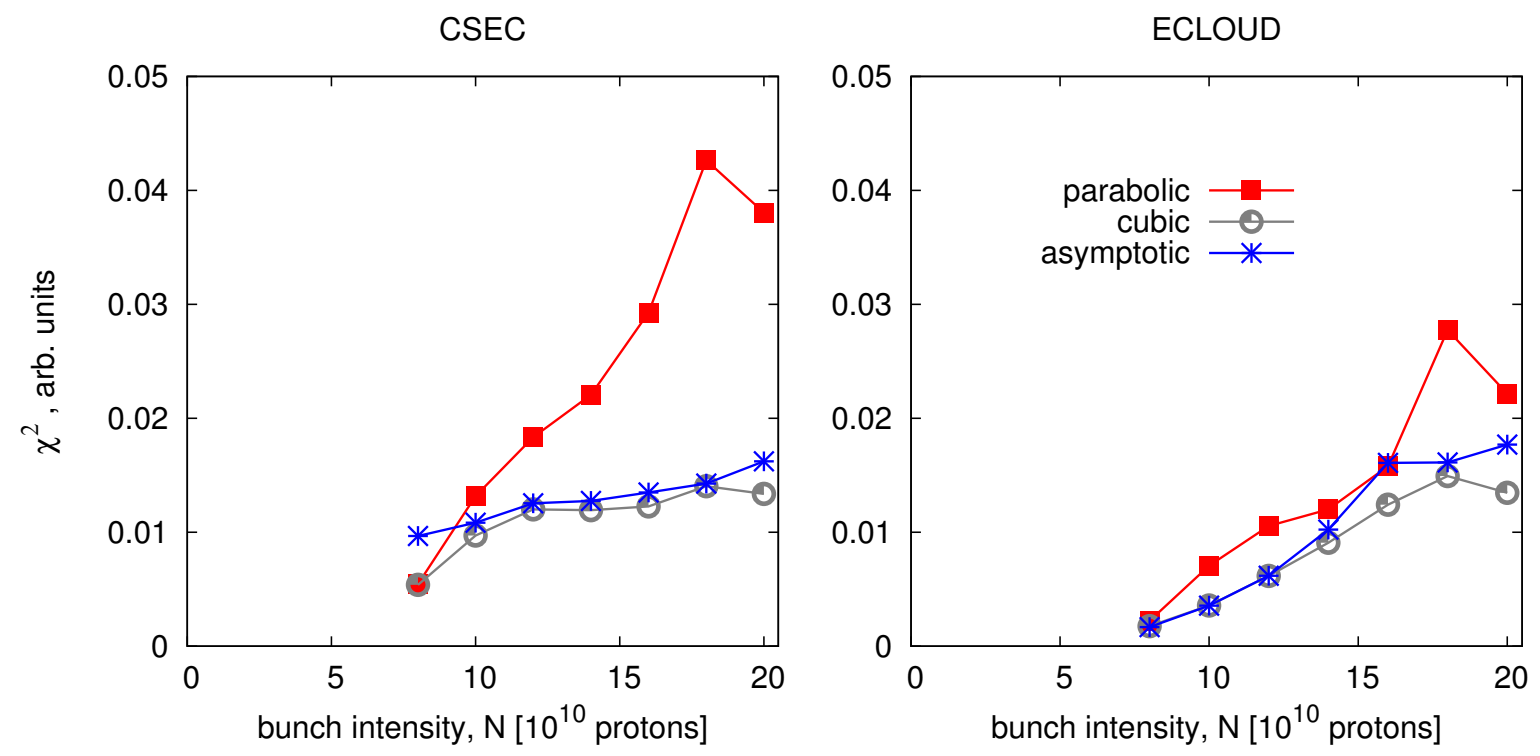

Figure 9.3: Evolution of the $\chi^{2}$ coefficient as a function of bunch intensity, $N$, for the different maps tested: parabolic, cubic, and asymptotic for CSEC (left plot) and ECLOUD (right). Smaller values of $\chi^{2}$ indicate a better quality fit.

Thus, for the parameters shown in Table 9.1, the electron density growth for a given bunch intensity is determined by a 3 -vector $\vec{A}(N)=(a, b, c)$, while decay is described by two 3 -vectors, one corresponding to the "first empty bunch", and a second vector for the rest of them. Later in the text, it is shown that two 3-vectors are required as well when the bunch pattern is not uniform. 
Figure 9.4 shows how the build-up coefficients $(a, b, c)$ evolve as a function of the bunch intensity, $N$, for both CSEC (grey points), and ECLOUD (red squares). Both codes give a similar phase transition threshold, $N_{C}$ around $7 \times 10^{10}$ protons, when $a\left(N_{C}\right)=1$. The linear coefficient, $a$, becomes larger than 1 when $N>N_{C}$, and increases linearly to a first approximation. In all cases (different $N$ ), and using both codes, the quadratic coefficient, $b$, is negative. This gives concavity to the electron cloud density evolution in the space $\left(\rho_{m}, \rho_{m+1}\right)$ and ensures a positive saturation value (see Eq. 9.4). The $b$ coefficient decreases (increases in absolute value) for CSEC results, but using ECLOUD $b$ only decreases for bunch intensities $N>12 \times 10^{10}$ protons. It is surprising that $b$ is not a monotonic function of the bunch intensity. The cubic coefficient, $c$, is positive and about one order of magnitude smaller than the linear term for $N>10^{10}$ protons. However, both codes differ significantly as we approach the bunch intensity threshold ( $N<10^{10}$ protons).

The behavior of these coefficients is not well understood from first principles: the determination of their values is purely empirical. The dependence of these coefficients on the bunch intensity, $N$, is derived from electron cloud simulation codes. Analytical expressions for them have not been found so far, except for the linear map coefficient (see Chapter 10).
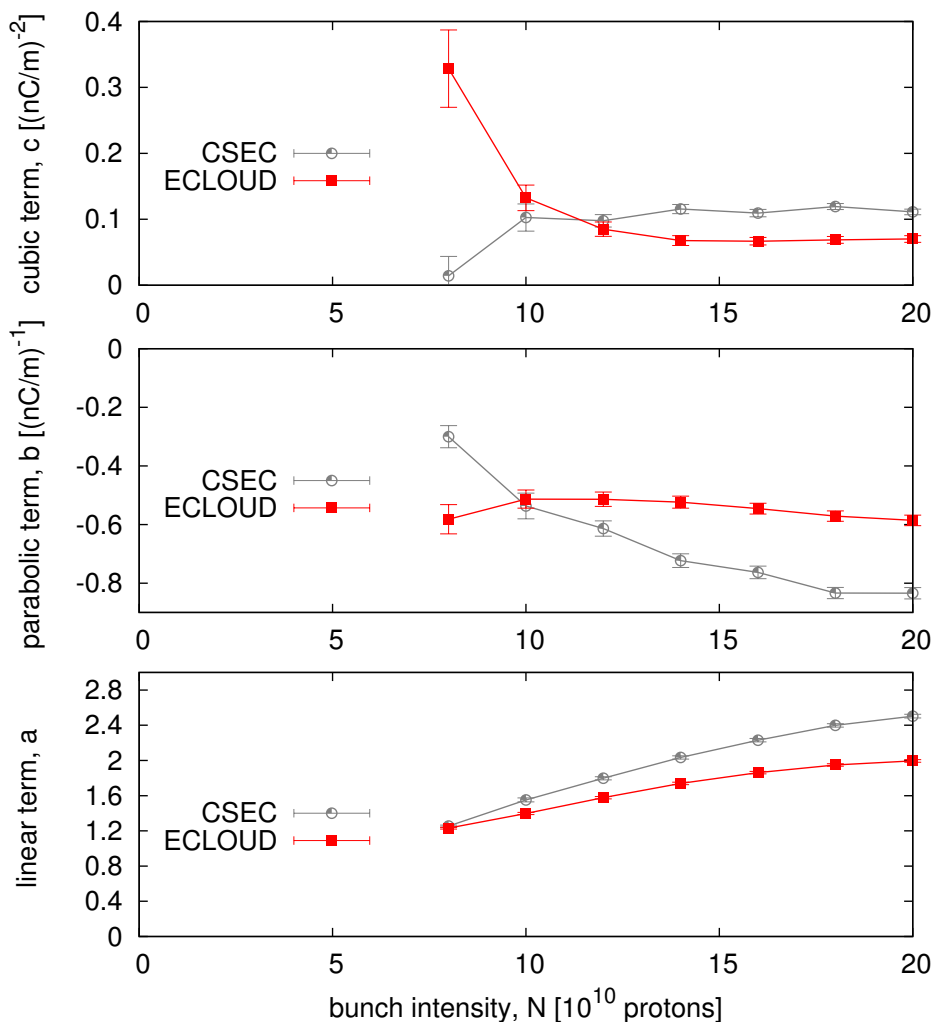

Figure 9.4: Evolution of the linear, $a$, parabolic, $b$, and cubic $c$ terms determining the electron cloud build up as a function of the bunch intensity, $N$, for both ECLOUD (red squares) and CSEC (grey points). 


\subsection{Minimization of electron density at RHIC}

After experimental observations in RHIC during Run-3 [82,90,104], it was found that gaps along the bunch train are useful against the electron cloud build up. Since the growth time is longer than the decay time (see, for example, Fig. 9.1) the goal is to find a bunch pattern around the RHIC circumference that does not trigger the electron cloud, or minimizes the detrimental effects of the phenomenon. In the following triplets of integers $\left(k_{s}, k_{b}, k_{g}\right)$ are used to describe bunch patterns. $k_{s}$ gives the bunch spacing in buckets (whose length is $36 \mathrm{~ns}), k_{b}$ the number of bunches filled with that spacing, and $k_{g}$ the number of "ghost" bunches added ("empty" bunches that are not filled in and therefore create a gap). Changing patterns can then be described by adding a new triplet. For example the configuration $(2,2,1)(3,4,0)$ would correspond to the pattern

$$
\text { 1-0-1-0-0-0-1-0-0-1-0-0-1-0-0-1-0-0 }
$$

where 1 denotes a filled and 0 denotes an empty bucket. If not otherwise noted, it is assumed that a pattern repeats until the abort gap is reached. RHIC has 360 buckets, and injection is allowed into every third bucket (minimum), with an abort gap of 30 buckets. This implies a maximum of 110 bunches.
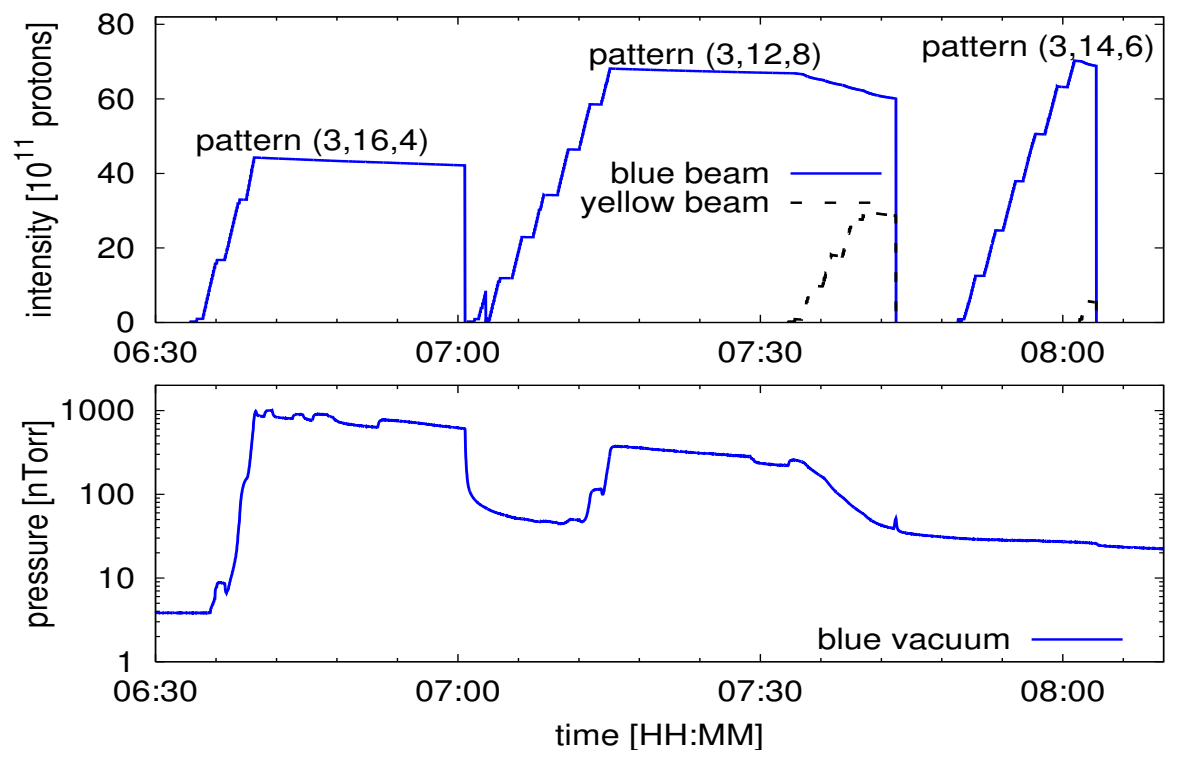

Figure 9.5: Attempts to fill RHIC with three different bunch patterns: $(3,16,4),(3,12,8)$, and $(3,14,6)$. The top plot shows the total intensity in the ring, while the bottom plot shows the pressure in one of the unbaked warm regions at RHIC (blue vacuum). Unlike the first attempt, $(3,16,4)$, the injection in the second case, $(3,12,8)$, does not prevent machine operation although the pressure rise is noticeable. The third case, $(3,14,6)$, is not relevant here due to an unusually large bunch length. 
Consider different distributions of 68 bunches. Figure 9.5 shows the result of three injection attempts with three different bunch patterns: $(3,16,4),(3,12,8)$, and $(3,14,6)$. Even though pressure rises are detected for the first two cases and not for the third one, (see pressure rise at the blue section in Fig. 9.5, bottom), we can inject up to 68 bunches using the configuration $(3,12,8)$, whereas injection of bunch pattern $(3,16,4)$ cannot be completed. A comparison among the three cases is complicated by the fact that bunch intensity and bunch length are not the same for all fills. Figure 9.5 also shows that the attempt to fill bunch pattern $(3,14,6)$ was successful, but it is not taken into account for this study because the bunch length was twice that of the previously attempted fills. Bunch intensity and length are comparable for fills $(3,16,4)$ and $(3,12,8)$, which also show similar vacuum behavior. On the other hand, the larger bunch lengths for fill $(3,14,6)$, together with the reduced bunch intensity, can account for the suppression of electron clouds. Table 9.2 summarizes the characteristics of the different cases and compares the relative luminosity.

Table 9.2: Comparison of bunch patterns tested in RHIC at injection.

\begin{tabular}{lccccc}
\hline \hline parameter & unit & $\begin{array}{c}\text { reference } \\
\text { case }\end{array}$ & $\begin{array}{c}\text { fill } \\
\text { no 1 }\end{array}$ & $\begin{array}{c}\text { fill } \\
\text { no 2 }\end{array}$ & $\begin{array}{c}\text { fill } \\
\text { no 3 }\end{array}$ \\
\hline bunch pattern & $\ldots$ & $(6,1,0)$ & $(3,16,4)$ & $(3,12,8)$ & $(3,14,6)$ \\
no of bunches & $\ldots$ & 56 & 41 & 69 & 78 \\
average proton/bunch & $10^{11}$ & 1.0 & 1.1 & 1.0 & 0.9 \\
total intensity & $10^{11}$ & 56.0 & 44.3 & 68.1 & 70.2 \\
full bunch length & $\mathrm{ns}$ & $\ldots$ & 16.5 & 17.6 & 34.2 \\
pressure rise & $\ldots$ & $\ldots$ & yes & yes & no \\
luminosity scaling factor & $\ldots$ & 1.00 & 0.88 & 1.23 & 1.13 \\
\hline \hline
\end{tabular}

Reference [90] studies the effect of the bunch pattern on the electron cloud. Several computer simulation runs were launched with different bunch patterns. The two criteria to minimize the effects of the electron cloud were the average, and the maximum value of the electron density created by each bunch pattern. The conclusion, consistent with the experience at B-factories [90], is that the most sparse distribution of bunches is the best way to optimize luminosity. However, since one CSEC run takes about $1 \mathrm{~h}$, if we want to study all the possibilities of distributing 68 bunches in 110 possible buckets, it is obvious that we cannot simulate

$$
\frac{110 !}{(110-68) ! 68 !} \approx 10^{30}
$$

different bunch distributions. Instead, electron cloud mpas are used in two ways to address the following question: given a fixed number of bunches and beam intensity, what is the optimum bunch distribution along the bunch train to minimize the electron cloud density? The first way is via the fast simulation MEC (Maps for Electron Cloud). The second is via a linear approximation, valid at small cloud densities. 


\subsubsection{Simulations for different bunch patterns}

The simulation code MEC uses a cubic interpolation map to follow the bunch to bunch evolution of the electron cloud density. Simulations of different bunch patterns carried out with CSEC and reported in [90] are compared using MEC in this section. The parameters used by CSEC are reported in Table 9.1, but in this case we fix the bunch intensity at $N=N_{0}=8 \times 10^{10}$ protons.

The use of MEC is divided into four cases, depending on the intensity of the bunches $m$ and $m-1$ passing by:

- First "full" bunch, which denotes a full bunch after an empty one, i.e. $N_{m}=N_{0}$ and $N_{m-1}=0$, with cubic map coefficients represented by the vector: $\vec{A}_{10}=\left(a_{10}, b_{10}, c_{10}\right)$.

- "Full" bunches, denoting the passage of a bunch with $N_{m}=N_{0}$ protons after another full bunch, $N_{m-1}=N_{0}$. The cubic map coefficients for this case are denoted by $\vec{A}_{11}=\left(a_{11}, b_{11}, c_{11}\right)$.

- First "empty" bunch, an empty bunch after a populated bunch, i.e. $N_{m}=0$ and $N_{m-1}=N_{0}$. The corresponding cubic map coefficients are represented by $\vec{A}_{01}=$ $\left(a_{01}, b_{01}, c_{01}\right)$.

- "Empty" bunches, succession of bunches with intensity $N_{m}=0$ and $N_{m-1}=0$. The corresponding cubic map coefficients are denoted by $\overrightarrow{A_{00}}=\left(a_{00}, b_{00}, c_{00}\right)$.

The need for this subdivision is justified by analysing two figures: in Fig. 9.2 one sees that the "first $N_{m}=0$ " is out of the evolution of decay curve, i.e. the curve corresponding to "ghost" bunches. Figure 9.6 justifies the case for the "first $N_{m}=N_{0}$ " curve. Figure 9.6 shows that the transition from "empty" to "full" also requires two bunches, in the same way that the transition from "full" bunch to "empty" bunch is done in two bunches.

One obtains successful results when comparing the bunch to bunch evolution using CSEC and MEC: see Fig. 9.7 with the different bunch patterns. Table 9.3 compares the maximum and average values for the linear electron cloud density at the last turn using CSEC and MEC. The largest difference for the maximum density is about 15\% (corresponding to the case $(3,2,0)(6,4,0))$; while for the average density the maximum difference is about $17 \%$, corresponding to the case $(3,23,17)$. While CSEC uses about $1 \mathrm{~h}$ CPU time for each case, MEC is obviously much faster and only uses $\sim 1 \mathrm{~ms}-$ a speed up of seven orders of magnitude! 


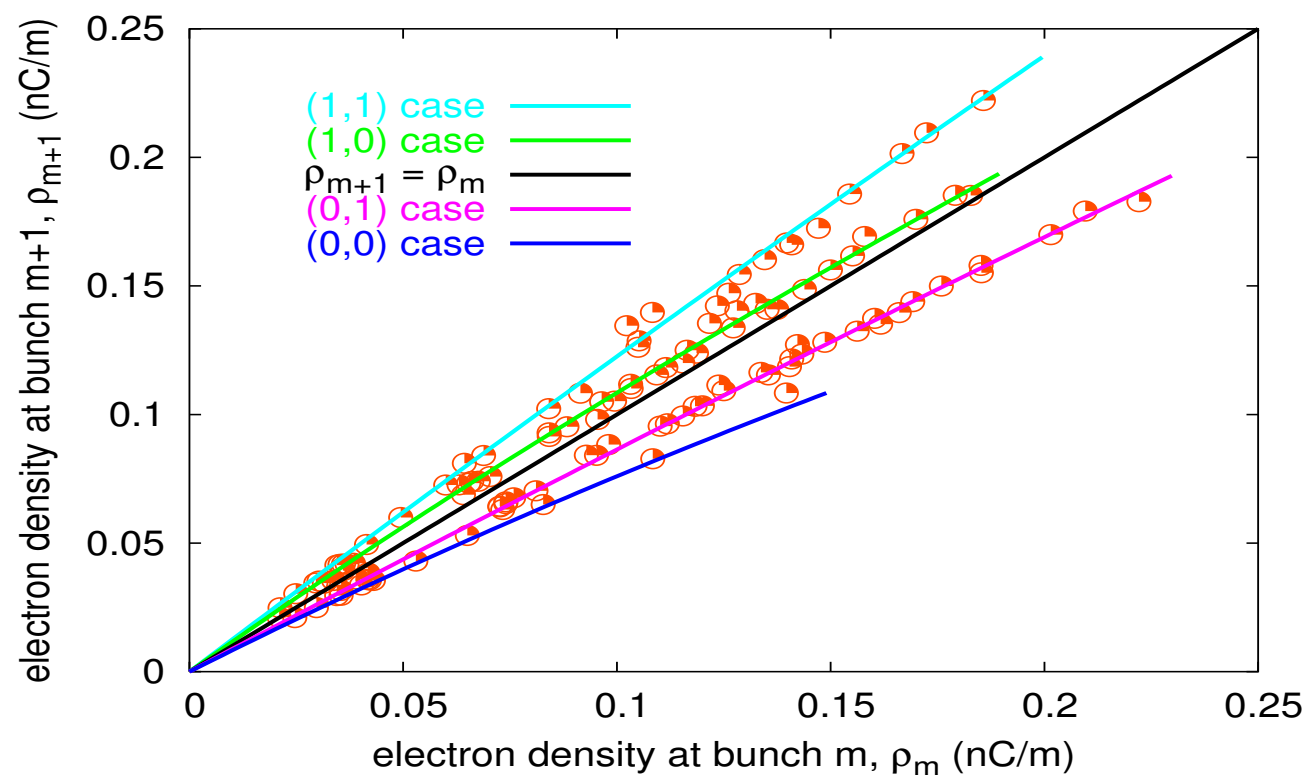

Figure 9.6: Electron cloud density in $\left(\rho_{m}, \rho_{m+1}\right)$ space for the bunch pattern $(3,4,0)(6,8,0)$ with $N_{0}=8 \times 10^{10}$ protons. The plot shows that four different behaviors are required: the case $(1,1)$ refers to "full" bunches preceded by another "full" bunch; the case $(1,0)$ refers to "full" bunches preceded by an "empty" bunch; the case $(0,1)$ to "empty" bunches preceded by a "full" bunch; while the case $(0,0)$ denotes a "empty" bunch preceded by another "empty" bunch.

Table 9.3: Maximum, $\rho_{\max }$, and average, $\rho_{\text {avg }}$, bunch to bunch values of the linear electron density simulated with CSEC and MEC for different bunch patterns. The results agree within about $15 \%$.

\begin{tabular}{lcccccc}
\hline \hline parameter & unit & $\begin{array}{c}\text { case } \\
\text { no 1 }\end{array}$ & $\begin{array}{c}\text { case } \\
\text { no } 2\end{array}$ & $\begin{array}{c}\text { case } \\
\text { no 3 }\end{array}$ & $\begin{array}{c}\text { case } \\
\text { no } 4\end{array}$ & $\begin{array}{c}\text { case } \\
\text { no 5 }\end{array}$ \\
\hline bunch pattern & $\ldots$ & $(3,68,52)$ & $(3,23,17)$ & $(3,12,8)$ & $(3,4,0)(6,8,0)$ & $(3,2,0)(6,4,0)$ \\
number of bunches & $\ldots$ & 68 & 68 & 68 & 68 & 68 \\
protons per bunch & $10^{10}$ & 8.0 & 8.0 & 8.0 & 8.0 & 8.0 \\
$\rho_{\max }$ using CSEC & $\mathrm{nC} / \mathrm{m}$ & 0.8991 & 0.6203 & 0.2849 & 0.2221 & 0.2033 \\
$\rho_{\max }$ using MEC & $\mathrm{nC} / \mathrm{m}$ & 0.9302 & 0.6645 & 0.2861 & 0.2184 & 0.2370 \\
$\rho_{\text {avg }}$ using CSEC & $\mathrm{nC} / \mathrm{m}$ & 0.3023 & 0.1433 & 0.0981 & 0.1006 & 0.0922 \\
$\rho_{\text {avg using MEC }}$ & $\mathrm{nC} / \mathrm{m}$ & 0.3216 & 0.1156 & 0.1045 & 0.0992 & 0.0924 \\
figure & - & - & $9.7, \mathrm{~A}$ & $9.7, \mathrm{~B}$ & $9.7, \mathrm{C}$ & $9.7, \mathrm{D}$ \\
\hline \hline
\end{tabular}



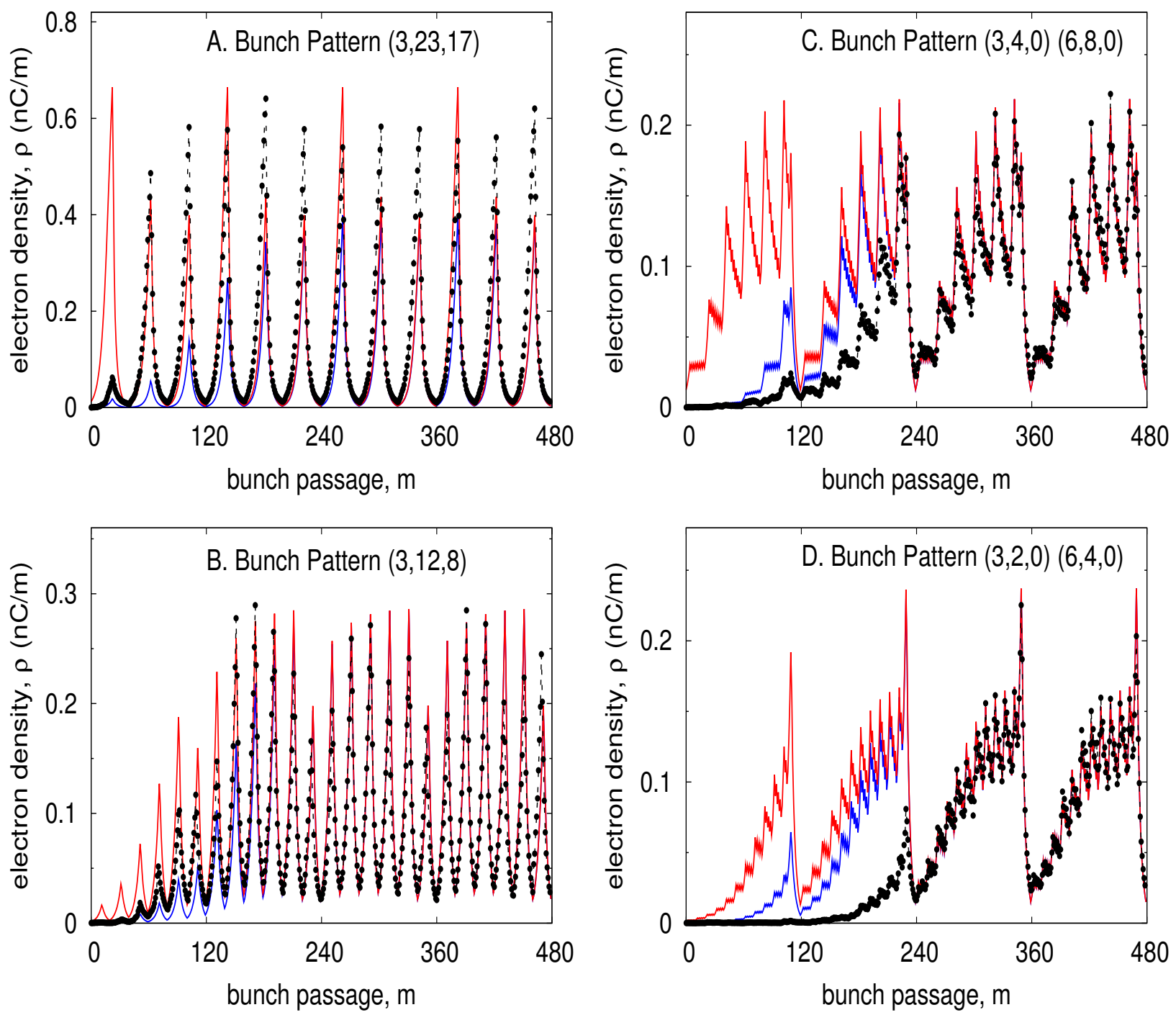

MEC, initial $\rho_{0}=10^{-4} \mathrm{nC} / \mathrm{m}-$
MEC, initial $\rho_{0}=10^{-3} \mathrm{nC} / \mathrm{m} \longrightarrow$
CSEC $\cdots$.

Figure 9.7: Electron cloud density evolution for four of the different bunch patterns in Table 9.3 using CSEC (dashed black trace with dots) and MEC with two different initial electron densities: $\rho_{0}=10^{-4} \mathrm{nC} / \mathrm{m}$ (blue line) and $\rho_{0}=10^{-3} \mathrm{nC} / \mathrm{m}$ (red line). No matter the initial electron density, MEC results agree for the last turn (from bunch passage 360 to 480) within an error range of $\approx 15 \%$ for all bunch patterns. Plots for bunch patterns $\mathrm{C}$ and $\mathrm{D}$ (on the right hand side) have the same vertical scale. Scales for A and B differ. 


\subsubsection{The linear approximation}

MEC requires four sets of polynomial coefficients, $\vec{A}_{11}(N), \vec{A}_{01}(N), \vec{A}_{00}(N)$, and $\vec{A}_{10}(N)$, to follow the bunch to bunch evolution of the electron cloud density. Figure 9.6 suggests that for small electron densities, the bunch to bunch evolution can be considered as linear in the $\left(\rho_{m}, \rho_{m+1}\right)$ space. That is, if there is a total number of $M$ bunches in a ring with a "bunch harmonic" number of $H$, the linearization of the problem gives a one turn map that is simply:

$$
\rho_{m+H} \approx F(N) \rho_{m}
$$

where the "one turn factor" is

$$
F \equiv\left(a_{10} a_{01}\right)^{i} a_{11}^{M-i} a_{00}^{H-M-i},
$$

and $i$ is the number of transitions from full to empty (and empty to full) bunches. In general the minimum possible number of transitions is $i=1$ (if all the bunches are clumped together), and the maximum number of transitions is the smaller of $M$ and $H-M$ (when the bunches are spread as sparsely as possible). The special case $i=0$ applies when there is no abort gap, $M=H$.

It is clear that if $F>1$ then the electron cloud density increases (to some saturated value), while if $F<1$ then the cloud disappears. When the one turn factor is rewritten as

$$
F=\left(\frac{a_{10} a_{01}}{a_{11} a_{00}}\right)^{i}\left(\frac{a_{11}}{a_{00}}\right)^{M} a_{00}^{H}
$$

it is clear that, for given $M, H$ and $N$, the smallest (largest) value of $F$ occurs for the largest (smallest) allowed value of $i$ if

$$
\left(\frac{a_{10} a_{01}}{a_{11} a_{00}}\right)<1
$$

and vice versa. Since Eq. 9.10 is valid for RHIC parameters, the most sparse distribution of a fixed number of fixed population bunches is the most stable against electron cloud growth.

Thus, from the mapping approach and using a linearized approximation we have demonstrated that the most sparse distribution of bunches in RHIC minimizes the detrimental effects of the multi-bunch electron cloud effects. This is not a big surprise if we consider the possibility of evenly distributed bunches, i.e. the same bunch spacing between all bunches. In this case, the most sparse distribution of bunches is equivalent to using a larger bunch spacing between them. However, Eq. 9.9 demonstrates that this is also valid for unevenly spaced bunches along a bunch train. This "rule of thumb" has been used in RHIC operations when deciding which bunch pattern to inject $[89,105]$.

\subsection{Summary}

Multi-bunch electron cloud build up at RHIC is modelled by simple maps. A third order polynomial map, denoted by the vector $\vec{A}=(a, b, c)$ optimally reproduces the bunch to 
bunch evolution. For a given vacuum chamber, these coefficients are a function of the beam parameters. The coefficients $(a, b, c)$ are empirically determined as a function of the bunch intensity, $N$, using the existing electron cloud simulations codes, like CSEC or ECLOUD.

When jumping back and forth from full to empty bunches, a memory of two bunches is found to be necessary. Therefore a complete algorithm requires four vectors: $\vec{A}_{11}, \vec{A}_{10}$, $\vec{A}_{00}$, and $\vec{A}_{01}$. A simulation program, MEC, uses these vectors to reproduce (within about $15 \%$ ) the evolution of the electron density in a bunch to bunch approximation. The CPU time used in this case is 7 orders of magnitude smaller than that used by the conventional electron simulation codes (CSEC or ECLOUD).

The importance of this analysis lies not only in the acceptable reproducibility of the results using MEC, but also in the ability to abstract the way to tackle electron clouds. This helps to deliver conclusions that would otherwise be difficult to obtain. For instance, using the linearized maps, actual values for the vectors analytically demonstrate that for the straight sections of RHIC, the most sparse distribution of bunches is the most stable against electron cloud formation, even when they cannot be evenly spaced. 


\section{Chapter 10}

\section{The linear map coefficient}

In Chapter 9 it is seen that the evolution of the electron density during an electron cloud formation can be reproduced using maps in the form $\rho_{m+1}=f\left(\rho_{m}\right)$. However, the function $f\left(\rho_{m}\right)$ depends on coefficients that are calculated from long electron cloud simulation runs. This Chapter presents an analysis to obtain the linear map coefficient from first principles. The results are compared with the linear coefficients obtained using CSEC. This analysis is useful for setting the thresholds upon which the electron cloud build up occurs, and shows how the resonance condition is not a sine qua non for the phenomenon.

\subsection{Introduction}

Chapter 3 describes two crucial ingredients for the calculation of the linear map coefficient. The first is the energy gained by an electron during the passage of a bunch with a non-uniform charge distribution (see Section 3.2.2). The second is the number of secondary electrons produced after an electron-wall collision as a function of the electron energy (parameterization of $\delta(E)$, see Section 3.3). The quantitative picture of how an electron cloud builds is then reduced to: 1) evaluate the energy gain due to a bunch passage, 2) compute the multiplication at the chamber wall, and 3) calculate the electrons surviving until the next bunch arrival. With some approximations, the three steps are calculated in the following. A similar calculation was first introduced in Ref. [67], although it did not include the survival time of the electrons.

Consider $N_{m}$ quasi-stationary electrons uniformly distributed in the transverse crosssection of the beam-pipe, as shown in Fig. 10.1. When a bunch passes by, electrons are accelerated to an energy $E$, and produce $\delta(E)$ secondary electrons when they hit the chamber wall. These secondary electrons still perform several wall collisions before the next bunch arrives, and then a total of $N_{m+1}$ electrons have survived. If $N_{m+1} / N_{m}>1$, the number of electrons increases with each bunch passage, leading to the development of a "cloud" of electrons. For small values of $N_{m}$ and $N_{m+1}$, the number of electrons at bunch passage $m$ grows exponentially

$$
N_{m+1}=a^{m} N_{0},
$$


where $a$ is defined as

$$
a=N_{m+1} / N_{m}
$$

This is equivalent to the linear map coefficient (independently of the map form - see Eqs. 9.1, 9.5, and 9.6). The unlimited growth predicted by Eq. 10.1 is eventually stopped by space charge effects, or, using map language, the influence of a second order terms.

In the following, a cilindrical beam pipe of radius $R_{p}$ is taken (RHIC case). In the absence of external electromagnetic fields, survival between two bunches is based on the fact that an electron can only "disappear" (with a certain probability) after a wall collision. It is useful to compute the electron time of flight $t_{F}$ as the time between two consecutive wall collisions and compare it with the bunch spacing $t_{\mathrm{sb}}$. Assuming that the electron motion is limited to the transverse radial direction (transverse plane, electron trajectories crossing along the beam pipe diameter), the time of flight is $t_{F}=2 R_{p} / v$, where $v$ is the electron speed. As a function of energy, this is

$$
t_{F}(E)=\frac{2 R_{p}}{\sqrt{2 E / m_{e}}},
$$

where $m_{e}$ is the electron mass. The assumption of transverse radial motion implies that the electron-wall collisions are at perpendicular incidence angle. Although this is not a bad approximation for field free regions, it is not valid for magnetic field regions (see for example, the electron motion in Fig. 8.3).

\subsubsection{Energy gain and first electron-wall collision}

The average energy gain of a uniformly distributed cloud of $N_{m}$ electrons in a cylindrical beam pipe of radius $R_{p}$ depends on the "critical radius". As seen in Section 3.2.2, this parameter is in the same order of the beam pipe radius for typical RHIC parameters, so the energy gain is given by the intermediate regime - Eq. 3.19. This expression is

$$
E_{g}=m_{e} c^{2} \frac{Z N_{b} r_{e}}{\sqrt{2 \pi} \sigma_{z}}\left(\ln \frac{R_{p}}{c_{0} \sigma_{r}}-\frac{1}{2}\right)
$$

Assume now that all the electrons initially at rest gain this energy after the bunch passage. This monoenergetic electron jet travels from the center of the beam pipe until the first wall collision (traveling a distance $R_{p}$ ). Using Eq. 10.3, this time is

$$
t_{F}\left(E_{g}\right) / 2=\frac{R_{p}}{\sqrt{2 E_{g} / m_{e}}} \equiv t_{\mathrm{FG}} / 2
$$

where $t_{\mathrm{FG}} \equiv t_{F}\left(E_{g}\right)$ denotes the time of flight for the electron jet with energy $E_{g}$. As a result of this first wall collision there are

$$
\begin{array}{lll}
N_{\mathrm{G} 1, \mathrm{sec}}=N_{m} \delta_{t}\left(E_{g}\right) \equiv N_{m} \delta_{t} & \text { true secondaries, and } \\
N_{\mathrm{G} 1, \mathrm{ref}}=N_{m} \delta_{r}\left(E_{g}\right) \equiv N_{m} \delta_{r} & \text { elastically reflected electrons, }
\end{array}
$$


corresponding to the two major processes occurred after an electron-wall collision (see Section 3.3): electrons that penetrate a few tens of nanometers into the material and are emitted at low energies (around $5 \mathrm{eV}$ ), and elastically reflected electrons, whose energy equals the energy of the incident particle. The symbol $G 1$ stands for the first collision of the electron jet with energy $E_{g}$. To avoid long mathematical expressions, the notation

$$
\begin{aligned}
\delta_{t}\left(E_{g}\right) & \equiv \delta_{t} \quad \text { and } \\
\delta_{r}\left(E_{g}\right) & \equiv \delta_{r}
\end{aligned}
$$

has been introduced. Now there are two jets to "track":

1. a high energy jet of $N_{\mathrm{G} 1 \text {,ref }}$ electrons, given by Eq. 10.7, whose energy $E=E_{g}$. In case multipacting takes place, $E_{g} \approx 200 \mathrm{eV}$ (depending on the electron-bunch interaction, Eq. 10.4). This jet is depicted with a bold black line in Fig. 10.1.

2. a low energy jet of $N_{\mathrm{G} 1 \text {, sec }}$ electrons, given by Eq. 10.6, whose energy $E=E_{\mathrm{sec}}$ is typically around $5 \mathrm{eV}$. This jet is depicted with a dotted red line in Fig. 10.1.

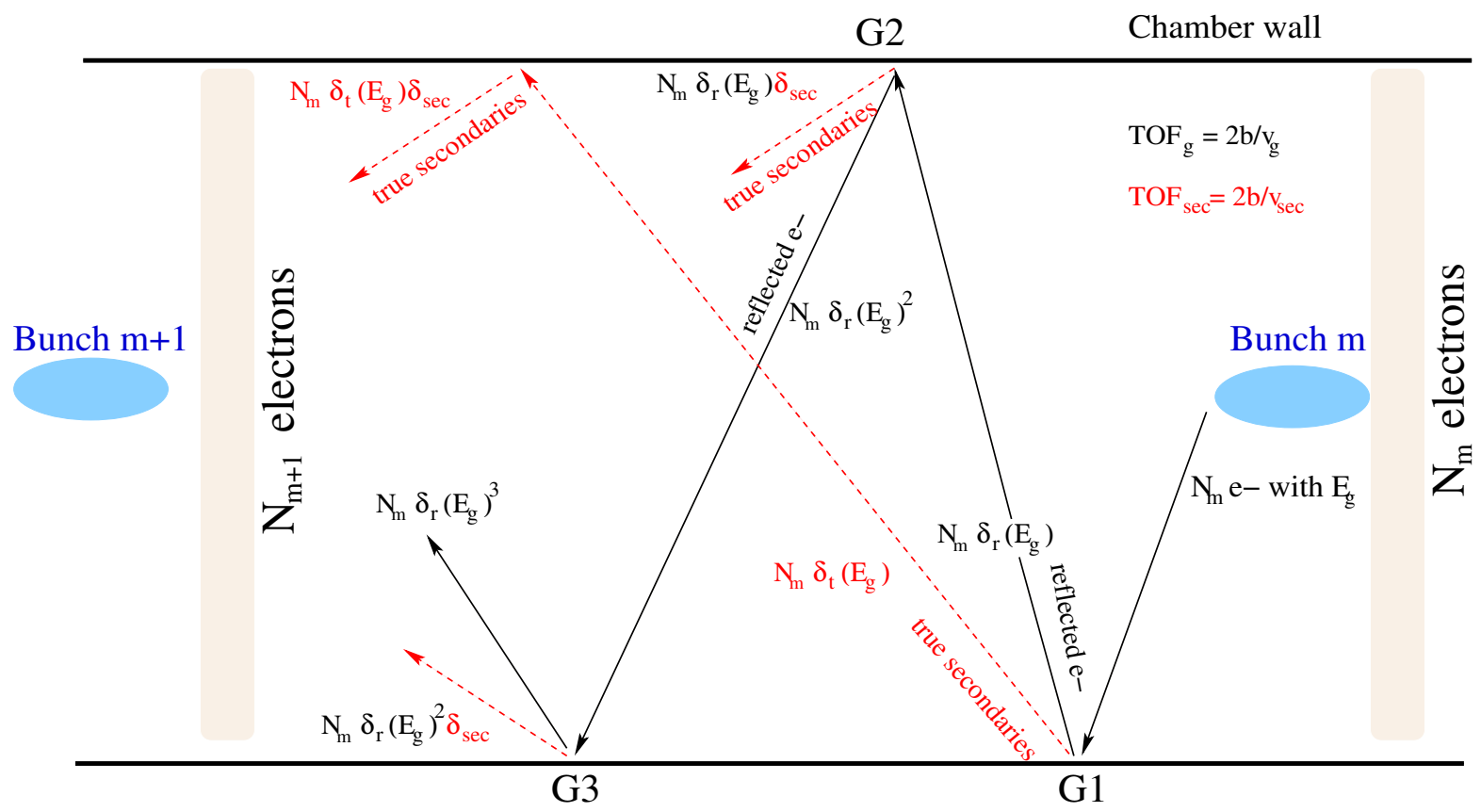

Figure 10.1: Schematic analysis of the evolution of an electron cloud between two bunch passages. The bunch $m$ arrives at the location where the $N_{m}$ electrons are uniformly distributed and initially at rest. After the bunch passage, electrons are accelerated towards the chamber wall with energy $E_{g}$ and have their first wall collision, $G 1$, when two new jets are created: one with energy $E_{g}$ and $N_{m} \delta_{r}$ electrons, corresponding to backscattered electrons (black line); the second with low energy and $N_{m} \delta_{t}$ electrons, corresponding to the true secondaries (red dotted line). Before bunch $m+1$ arrives, these two jets perform several wall collisions, which in turn create more jets. The contribution of all these jets becomes the number of surviving electrons, $N_{m+1}$. 
The contribution of the so-called "rediffused" electrons is neglected in this analysis. These are emitted at intermediate energies, i.e. between $E_{\mathrm{sec}}$ and $E_{g}$ (see Fig. 10.2, and Section 3.3), corresponding to random processes. Figure 10.2 shows a comparison between the measured energy distribution curve (red points) and the energy distribution curve assumed in this analysis (blue boxes). The energy distribution curves in this case becomes two Dirac delta functions centered at $E_{\mathrm{sec}}$ and $E_{g}$, whose height is proportional to $\delta_{t}$ and $\delta_{r}$, respectively.
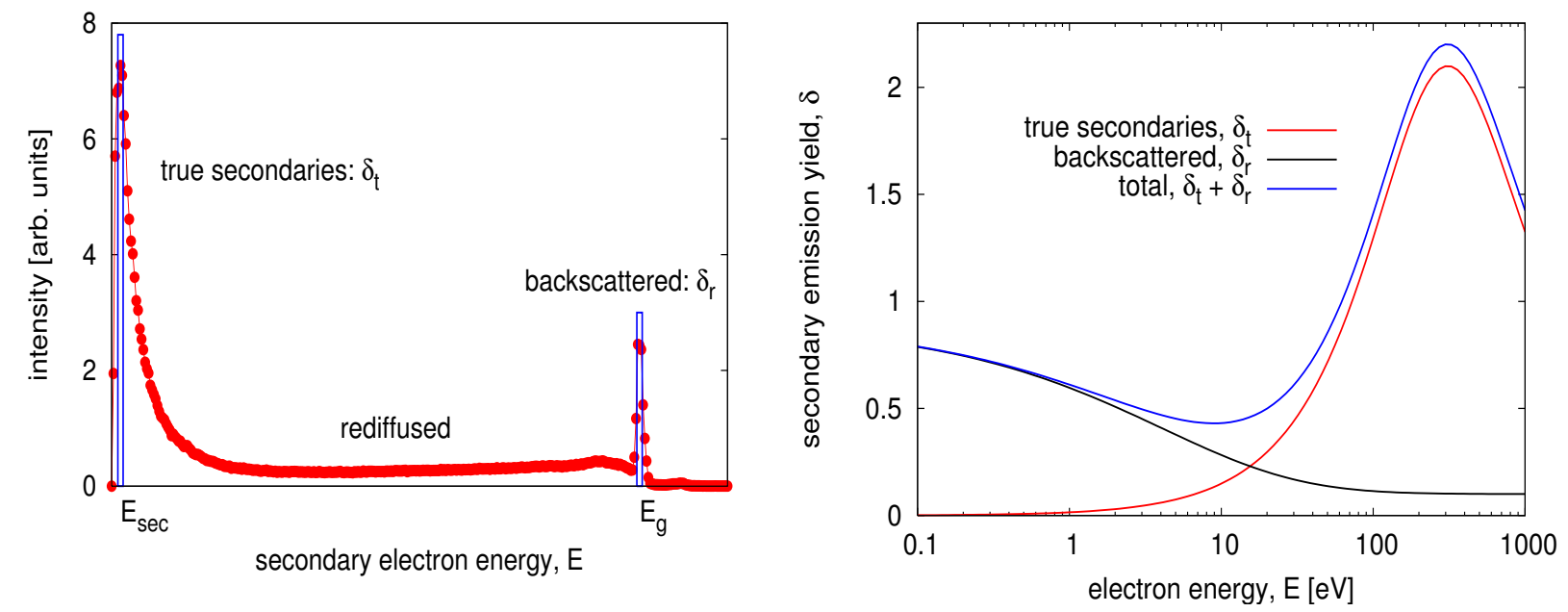

Figure 10.2: Left: The red points show the measured energy distribution curve in the secondary electron emission process: true secondaries, rediffused, and backscattered (see Fig. 3.3). The blue boxes show the energy distribution curve used for this analysis: the backscattered electrons are emitted with the same energy the primary electrons had when they impacted the wall (called $E_{g}$ in the text). The true secondaries are emitted with the same energy, $E_{\mathrm{sec}}$. The rediffused electrons are neglected in this approximation. Right: Secondary emission yield as a function of the primary electron energy. The total SEY (or $\delta$ ) accounts for the contribution of the "true secondaries" $\left(\delta_{t}\right.$, emitted with an energy $E_{\mathrm{sec}}$ ) and the elastically reflected (or backscattered, $\delta_{r}$, emitted with energy $\left.E_{g}\right)$.

\subsubsection{High energy electron-wall collisions}

The evolution of the elastically reflected electrons after the first collision, $N_{\mathrm{G} 1 \text {,ref }}$ in Eq. 10.7 are first followed. Considering the time origin at the bunch $m$ passage, these electrons have a second wall impact $G 2$ at

$$
\frac{t_{\mathrm{FG}}}{2}+t_{\mathrm{FG}}=\frac{3}{2} t_{\mathrm{FG}}
$$

producing

$$
\begin{array}{ll}
N_{\mathrm{G} 2 \text {,ref }}=N_{\mathrm{G} 1, \mathrm{ref}} \delta_{r}=N_{m} \delta_{r} \delta_{r} & \text { elastically reflected electrons, and } \\
N_{\mathrm{G} 2 \text {,sec }}=N_{\mathrm{G} 1, \mathrm{ref}} \delta_{t}=N_{m} \delta_{r} \delta_{t} & \text { true secondaries. }
\end{array}
$$


The elastically reflected electrons $\left(N_{\mathrm{G} 2 \text {,ref }}\right.$ in Eq. 10.11) have another collision (marked with G3 in Fig. 10.1) at

$$
\frac{t_{\mathrm{FG}}}{2}+t_{\mathrm{FG}}+t_{\mathrm{FG}}=\frac{5}{2} t_{\mathrm{FG}}
$$

again producing elastically reflected electrons (similar to in Eq. 10.11), and "true secondaries" (similar to Eq. 10.12). At Gi collision, the electrons leaving the wall are

$$
\begin{array}{ll}
N_{\mathrm{Gi}, \mathrm{ref}}=N_{m} \delta_{r}^{i} & \text { elastically reflected electrons, and } \\
N_{\mathrm{Gi}, \mathrm{sec}}=N_{m} \delta_{r}^{i-1} \delta_{t} & \text { true secondaries. }
\end{array}
$$

\subsubsection{Low energy electron-wall collisions}

For low energy electrons impinging on a surface, there is no fundamental distinction between true secondaries and elastically reflected electrons. All secondary electrons are considered to have been produced after elastic processes (see Section 3.3). Thus, define

$$
\delta\left(E_{\mathrm{sec}}\right)=\delta_{t}\left(E_{\mathrm{sec}}\right)+\delta_{r}\left(E_{\mathrm{sec}}\right) \equiv \delta_{\mathrm{sec}},
$$

insofar as the secondary electrons are all emitted with the same energy $E_{\mathrm{sec}} \sim 5 \mathrm{eV}$.

\section{Number of low energy electrons after the $G 1$ collision:}

After the first wall collision ( $G 1$ in Fig. 10.1), the emitted true secondary electrons $\left(N_{m} \delta_{t}\right.$ in Eq. 10.6) perform $k 1$ wall collisions with energy $E_{\mathrm{sec}}$ between the time of the first collision and the passage of the next bunch:

$$
k_{1}=\frac{t_{\mathrm{sb}}-t_{\mathrm{FG}} / 2}{t_{\mathrm{sec}}},
$$

where

$$
t_{\mathrm{sec}} \equiv t_{F}\left(E=E_{\mathrm{sec}}\right)
$$

is the time of flight for the true secondary electrons. The number of electrons surviving until the next bunch arrives is:

$$
N_{k_{1}}=N_{\mathrm{G} 1, \mathrm{sec}} \delta_{\mathrm{sec}}^{k_{1}}=N_{m} \delta_{t} \delta_{\mathrm{sec}}^{k_{1}}
$$

\section{Number of low energy electrons after the $G 2$ collision:}

Next, add the contribution from the true secondary electrons created after the $G 2$ collision (see Fig. 10.1). These are the true secondary electrons created by the elastically reflected jet produced after the first wall collision, G1. At the time of its creation, this jet has

$$
N_{\mathrm{G} 2, \mathrm{sec}}=N_{m} \delta_{r} \delta_{t}
$$


electrons with energy $E_{\mathrm{sec}}$. The number of collisions this jet has before the bunch $m+1$ arrives is

$$
k_{2}=\frac{t_{\mathrm{sb}}-3 t_{\mathrm{FG}} / 2}{t_{\mathrm{sec}}},
$$

and thus the number of electrons surviving until the next bunch arrives is

$$
N_{k_{2}}=N_{\mathrm{G} 2, \mathrm{sec}} \delta_{\mathrm{sec}}^{k_{2}}=N_{m} \delta_{r} \delta_{t} \delta_{\mathrm{sec}}^{k_{2}}
$$

\section{Number of low energy electrons after the $G i$ collision:}

This process is extrapolated to compute the surviving true secondary electrons created after the $G i^{\text {th }}$ collision,

$$
N_{k_{i}}=N_{m} \delta_{r}^{i-1} \delta_{t} \delta_{\mathrm{sec}}^{k_{i}}
$$

where $k_{i}$ is the number of collisions for the jet with $N_{\mathrm{Gi}, \mathrm{sec}}$ electrons at energy $E_{\mathrm{sec}}$,

$$
k_{i}=\frac{t_{\mathrm{sb}}-(2 i-1) t_{\mathrm{FG}} / 2}{t_{\mathrm{sec}}} .
$$

\subsubsection{Total survival of low and high energy electron-wall colli- sions}

The total number of electrons surviving between two bunch passages is computed by adding up the contribution from the elastically reflected and the true secondary electrons. The elastically reflected electrons surviving are just the ones coming after the last $G n$ collision:

$$
N_{\mathrm{Gn}, \mathrm{ref}}=N_{m} \delta_{r}^{n},
$$

where $n$ is the number of collisions for the monoenergetic jet with energy $E_{g}$ :

$$
n=\frac{t_{\mathrm{sb}}-t_{\mathrm{FG}} / 2}{t_{\mathrm{FG}}}
$$

The surviving true secondaries are the summation of the contribution by all the true secondaries created after each $G i$ collision:

$$
\sum_{i=1}^{n} N_{k_{i}}=N_{m} \delta_{t} \sum_{i=1}^{n} \delta_{r}^{i-1} \delta_{\mathrm{sec}}^{k_{i}}
$$

Using Eqs. 10.24 and 10.26, $k_{i}$ can be further simplified as:

$$
k_{i}=(n+1-i) \xi
$$

where the parameter

$$
\xi \equiv \sqrt{E_{\mathrm{sec}} / E_{g}}
$$


has been introduced for convenience. The contributions of both the surviving reflected electrons (Eq. 10.25) and all the true secondaries (Eq. 10.27) provides the number of electrons before bunch $m+1$ arrives:

$$
N_{m+1}=N_{m}\left[\delta_{r}^{n}+\delta_{t} \sum_{i=1}^{n} \delta_{r}^{i-1} \delta_{\mathrm{sec}}^{(n+1-i) \xi}\right]
$$

Hence, the linear map coefficient $a$ is

$$
a=\delta_{r}^{n}+\delta_{t} \sum_{i=1}^{n} \delta_{r}^{i-1} \delta_{\mathrm{sec}}^{(n+1-i) \xi}=\delta_{r}^{n}+\delta_{t} \frac{\delta_{\mathrm{sec}}^{(n+1) \xi}}{\delta_{r}} \sum_{i=1}^{n}\left(\frac{\delta_{r}}{\delta_{\mathrm{sec}}^{\xi}}\right)^{i},
$$

which can be further simplified to

$$
a=\delta_{r}^{n}+\delta_{t} \delta_{\mathrm{sec}}^{\xi} \frac{\delta_{\mathrm{sec}}^{n \xi}-\delta_{r}^{n}}{\delta_{\mathrm{sec}}^{\xi}-\delta_{r}} .
$$

Recall that the terms in $\delta_{t}, \delta_{r}, n$, and $\xi$ are functions of the energy gain produced by the bunch passage (parameter $E_{g}$, see Eq. 10.4), while $n$ is also a function of the bunch spacing as well. Thus, Eq. 10.32 merges beam and wall surface chamber parameters in a single expression.

This calculation of $a$ has been performed assuming only one monoenergetic jet of energy $E_{g}$ results from the electron-bunch interaction. More realistic calculations should involve not a single jet with energy $E_{g}$ but the distribution of the energy spectrum $h(E)$. In this case, the general expression becomes

$$
a=\int_{0}^{\infty}\left[\delta_{r}(E)^{n(E)}+\delta_{t}(E) \delta_{\mathrm{sec}}^{\xi(E)} \frac{\delta_{\mathrm{sec}}^{n(E) \xi(E)}-\delta_{r}^{n(E)}}{\delta_{\mathrm{sec}}^{\xi(E)}-\delta_{r}(E)}\right] h(E) d E .
$$

From this expression, it is compelling to call the parameter $a$ the effective secondary emission yield of the beam pipe wall, $\delta_{\text {eff }}$, depending on both the chamber material and the beam characteristics.

\section{Limit of long bunch spacing and $\delta_{0} \rightarrow 1$}

A long held understanding about the electron cloud is that multi-bunch multipacting is not triggered with "long" bunch spacings. It is easy to mathematically prove this using Eq. 10.32. Take the case of an infinitely long bunch spacing $t_{\mathrm{sb}} \rightarrow \infty$. Using Eq. 10.26, it follows

$$
\lim _{t_{\mathrm{sb}} \rightarrow \infty} n \rightarrow \infty
$$

that is to say, the number of collisions tends to infinity. In this case, the effective secondary emission yield $\delta_{\text {eff }}$ becomes

$$
\begin{array}{r}
\lim _{n \rightarrow \infty} a=\lim _{n \rightarrow \infty}\left[\delta_{r}^{n}+\delta_{t} \delta_{\mathrm{sec}}^{\xi} \frac{\delta_{\mathrm{sec}}^{n \xi}-\delta_{r}^{n}}{\delta_{\mathrm{sec}}^{\xi}-\delta_{r}}\right]= \\
\lim _{n \rightarrow \infty} \delta_{r}^{n}+\delta_{t} \delta_{\mathrm{sec}}^{\xi} \lim _{n \rightarrow \infty} \frac{\delta_{\mathrm{sec}}^{n \xi}}{\delta_{\mathrm{sec}}^{\xi}-\delta_{r}}-\delta_{t} \delta_{\mathrm{sec}}^{\xi} \lim _{n \rightarrow \infty} \frac{\delta_{r}^{n}}{\delta_{\mathrm{sec}}^{\xi}-\delta_{r}} \rightarrow 0
\end{array}
$$


which forbids electron cloud formation. (Recall that $\delta_{r}<1$, and $\delta_{\text {sec }}<1$, since these terms refer to the backscattered probability - see Fig. 10.2, right).

However, latest experimental results show $\delta_{\text {sec }} \rightarrow 1$ (see Fig. 3.5 and Ref. [47]). In this case, the second summand in Eq. 10.35 does not vanish:

$$
\lim _{n \rightarrow \infty}\left(\lim _{\delta_{\mathrm{sec}} \rightarrow 1} a\right)=\lim _{n \rightarrow \infty}\left(\lim _{\delta_{\mathrm{sec}} \rightarrow 1} \delta_{t} \delta_{\mathrm{sec}}^{\xi} \frac{\delta_{\mathrm{sec}}^{n \xi}}{\delta_{\mathrm{sec}}^{\xi}-\delta_{r}}\right)=\frac{\delta_{t}}{1-\delta_{r}} .
$$

This shows that multipacting is triggered when

$$
\delta_{t}(E) /\left(1-\delta_{r}(E)\right)>1,
$$

(independently of the bunch spacing!). This result gives a crucial importance to the initial assumption, $\delta_{\sec } \rightarrow 1$. Unfortunately, due to technical limitations there is a significant uncertainty in the SEY measurements of low energy electrons, that is, $\delta_{\text {sec }}[6,47]$.

\section{Limitations}

This analysis has been restricted to field free regions, as a consequence of the consideration of straight line electron trajectories and perpendicular electron-wall collisions. The model is also restricted to situations in which:

- The energy gain is below the energy at which the maximum SEY occurs: $E_{g} \leq E_{\max }$. This is a consequence of neglecting the contribution of the rediffused electrons. The rediffused electrons created after an electron-wall collision at $E_{g} \gg E_{\max }$ have a significant influence since they may fall in the region where SEY has its maximum, $E_{\max }$. For RHIC, this excludes the cases with large bunch intensities (above $\approx 15 \times 10^{10}$ protons or equivalent ion charge) and short bunch lengths (below $\sigma_{z} \approx 0.5 \mathrm{~m}$ ).

- The bunch spacing should be at least twice the time of flight of the high energy electrons, $n \geq 2$.

This stems from the assumption that the initial $N_{m}$ electrons were at rest. From Eq. 10.31, it follows that the fraction of the $N_{m+1}$ electrons (after bunch passage $m$ ) with energies larger than $E_{\text {sec }}$ is proportional to $\delta_{r}(E)^{n}$. Since

$$
\delta_{r}(E) \approx 0.1 \quad ; \quad \forall E \gg E_{\mathrm{sec}},
$$

and this proportion is neglectable for $n>2$. Otherwise, at bunch passage $m+2$, there will be a significant fraction of electrons with energy $E_{g}$ that obtain a similar energy gain, which can result in a larger SEY when they strike the wall. For RHIC, this excludes bunch spacings shorter than $72 \mathrm{~ns}$.

\subsection{Application to RHIC}

As an example take the case of a stainless steel beam pipe in RHIC with an average energy gain $E_{g}=160 \mathrm{eV}$, and assume $n=1$. From the SEY parameters in Table 10.1,

$$
a=\delta_{\mathrm{eff}}=\delta_{r}+\delta_{t} \delta_{\mathrm{sec}}^{\xi}=1.79,
$$


which approaches $\delta\left(E_{g}\right)=1.8$. This corresponds to the resonance condition in Ref. [4,26]: time of flight equals the time between bunches and $\delta>1$ at wall impact. This resonance picture has been widely used to explain electron cloud formation. However, Eq. 10.31 proves that it is not a sine qua non because it neglects the survival of the low energy electrons.

A typical value for RHIC is $n \approx 6$ (corresponding to an energy $E_{g}=160 \mathrm{eV}$ ). Equation 10.31 now becomes

$$
a=\delta_{\mathrm{eff}}=\delta_{r}^{6}+\delta_{t} \delta_{\mathrm{sec}}^{\xi} \frac{\delta_{\mathrm{sec}}^{6 \xi}-\delta_{r}^{6}}{\delta_{\mathrm{sec}}^{\xi}-\delta_{r}}=1.42 .
$$

It is useful to study the circumstances under which $a=1$, corresponding to the electron cloud formation threshold. Table 10.1 lists typical values for RHIC regarding beam and chamber parameters. A scan in six different parameters is shown in Fig. 10.3. For each scan, the rest of the parameters correspond to the reference case in Table 10.1.

Table 10.1: Beam and SEY parameters used for the calculations in Fig. 10.3.

\begin{tabular}{lccccc}
\hline \hline parameter & symbol & unit & reference & scan range & Figure \\
\hline beam pipe radius & $b$ & $\mathrm{~cm}$ & 6 & $1.2-6$ & $10.3, \mathrm{a}$ \\
bunch spacing & $t_{\mathrm{sb}}$ & $\mathrm{ns}$ & 108 & $72-324$ & $10.3, \mathrm{~b}$ \\
maximum secondary emission yield & $\delta_{\max }^{*}$ & $\ldots$ & 1.9 & $1.3-2.5$ & $10.3, \mathrm{c}$ \\
secondary emission yield for $E \rightarrow 0$ & $\delta_{0}$ & $\ldots$ & 0.7 & $0.2-1$ & $10.3, \mathrm{~d}$ \\
bunch charge & $N_{b}$ & $e \times 10^{10}$ & 10 & $2-20$ & $10.3, \mathrm{e}$ \\
rms bunch length & $\sigma_{z}$ & $\mathrm{~m}$ & 1 & $0.5-3$ & $10.3, \mathrm{f}$ \\
energy of the true secondaries & $E_{\mathrm{sec}}$ & $\mathrm{eV}$ & 5 & $\ldots$ & $\ldots$ \\
reflection probability at $E \rightarrow \infty$ & $\delta_{\infty}$ & $\ldots$ & 0.15 & $\ldots$ & $\ldots$ \\
energy for max. SEY & $E_{\max }$ & $\mathrm{eV}$ & 300 & $\ldots$ & $\ldots$ \\
rms transverse beam size & $\sigma_{r}$ & $\mathrm{~mm}$ & 2 & $\ldots$ & $\ldots$ \\
reflection energy & $E_{r}$ & $\mathrm{eV}$ & 60 & $\ldots$ & $\ldots$ \\
energy exponent & $s$ & $\ldots$ & 1.83 & $\ldots$ & $\ldots$ \\
\hline \hline
\end{tabular}

Figure 10.3 (a) shows that larger beam pipe radius results in a gradual increase of $a$. A smaller beam pipe radius produces more electron-wall collisions, hence the electron survival is reduced. Note that the dependence is proportional to $\ln R_{p}$, as in Eq. 3.19. This shows that a "cheap" counter-measure for the electron cloud in RHIC is to reduce the beam pipe radius, consistent with resuts in [106]. As expected, $a$ decreases with increasing bunch spacings - see Fig. 10.3 (b). Figure 10.3 (c) shows how $a$ increases significantly with small changes on $\delta_{\max }$. Perhaps the most relevant influence is due to $\delta_{0}$. Figure 10.3 (d) shows that for $\delta_{0}<0.5$ it is very hard to obtain values $a>1$, and that $a$ increases to $a \simeq 2$ for $\delta_{0} \rightarrow 1$. Finally, the dependence of $a$ on the beam parameters (bunch charge - Fig. 10.3 (e), and rms bunch length - Fig. 10.3 (f)) follows the dependence on Eq. 3.19 in the range where the approximation is valid $\left(N<15 \times 10^{10}\right.$ protons, and $\left.\sigma_{z}<0.5 \mathrm{~m}\right)$.

All in all, Fig. 10.3 shows how maps become a useful tool for exploring the electron cloud evolution in parameter space. 

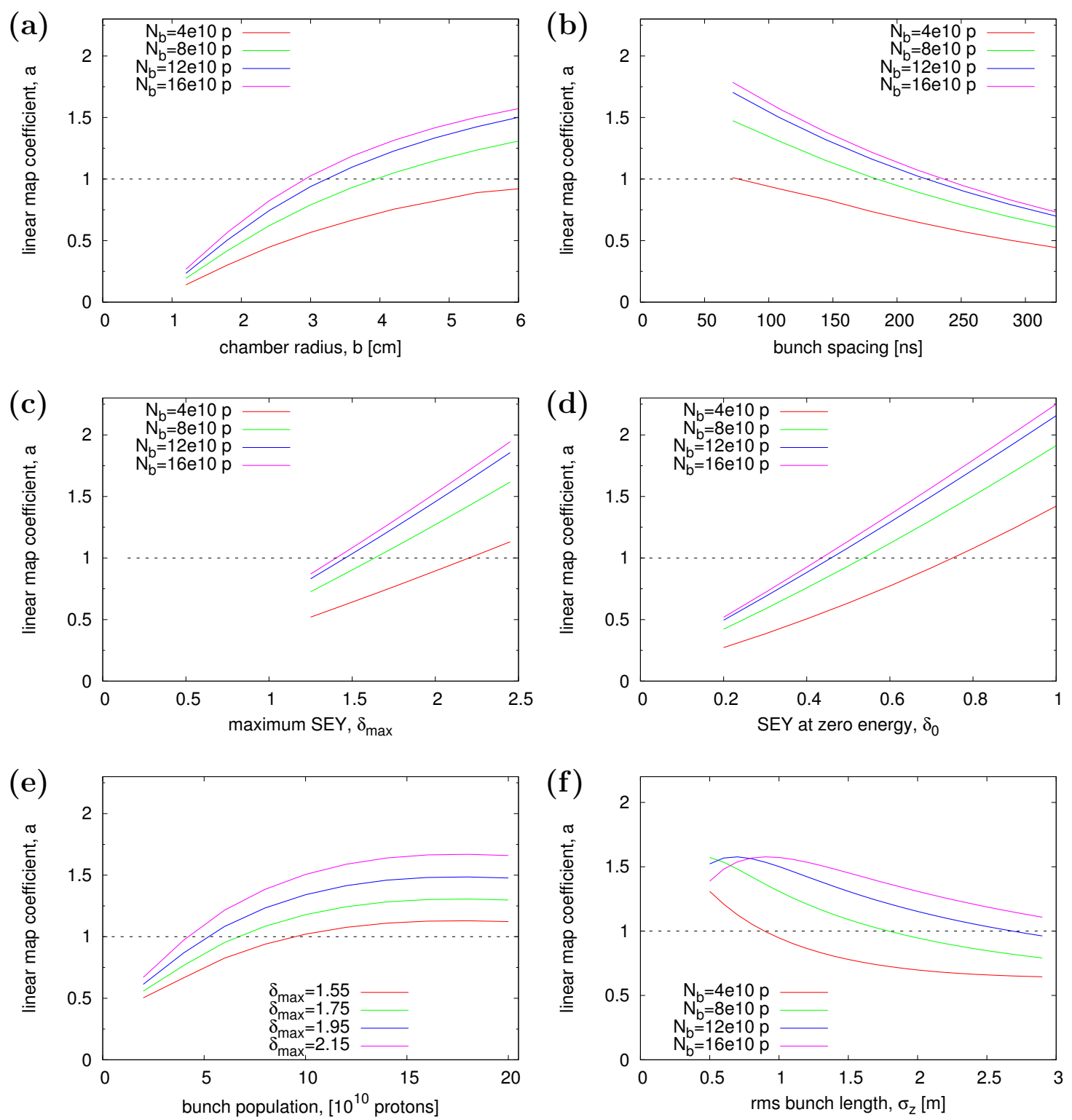

Figure 10.3: Analytical prediction of the influence of different beam and chamber parameters on electron cloud build up. Depicted is $a=N_{m+1} / N_{m}$ as a function of: (a) chamber radius, (b) bunch spacing, (c) maximum value of the SEY, (d) reflection probability at zero energy, (e) bunch population, and (f) bunch length. The value $a=1$ sets the threshold for electron cloud build up. 


\subsection{Comparison with simulation codes}

The map coefficients shown in Chapter 9 (obtained using CSEC and ECLOUD) are only calculated for bunch intensities above the electron cloud threshold, $N_{C}$. For a proper comparison between the simulation codes and the method used in Section 10.1, it is necessary to obtain the map coefficients for a whole range of parameters.

For bunch intensities below $N_{C}$, since the electron cloud does not build up, there is no data to fit. Two different simulations are launched to calculate the evolution of the map coefficients for a given value of bunch population $N$. These two simulations are:

1. a bunch train with the first 40 bunches populated with $N_{1}>N_{C}$, followed by 80 bunches with $N<N_{1}$ protons/bunch. An example for this simulation is shown in the top plot of Fig. 10.4 (left), where 40 bunches with $N_{1}=25 \times 10^{10}$ protons are followed by 80 bunches with $N=10 \times 10^{10}$ protons. The evolution of the electron density produced by this bunch pattern is shown by the red trace in the middle plot of Fig. 10.4 (left).

2. a bunch train of 80 bunches with $N$ protons per bunch. An example for $N=10 \times 10^{10}$ of this simple bunch pattern is shown in the bottom plot of Fig. 10.4 (left). The corresponding evolution of the electron density with the bunch passage is shown in the middle plot of of Fig. 10.4 (left). Note that the values for the final saturated electron density do not depend on the initial electron conditions.

Next, the points with the same bunch intensity are grouped together and plotted in the $\left(\rho_{m}, \rho_{m+1}\right)$ space, as shown in Fig. 10.4 (right). Note that values above or below the identity map lie on a general common curve. Thus, the fit to these points provides the values of the map coefficients for a given bunch intensiy, regardless of whether this bunch intensity is above or below the threshold. The fits correspond to second order map, third order map, and the assymptotic map (see Eqs. 9.1, 9.5, and 9.6). The left plot in Fig. 10.4 shows that the cubic fit is again slightly better, but small differences are found for the three fits. Figure 10.5 shows the cubic map coefficients as a function of the bunch intensity for the different $\delta_{\max }$.

In the following, the values of the linear map coefficient obtained using Eq. 10.31 are compared with CSEC results following the above method. This comparison is performed for a range of bunch intensities (from 0 to $20 \times 10^{11}$ protons), and for different $\delta_{\max }$. Table 10.2 shows the parameters used in the CSEC simulations. 


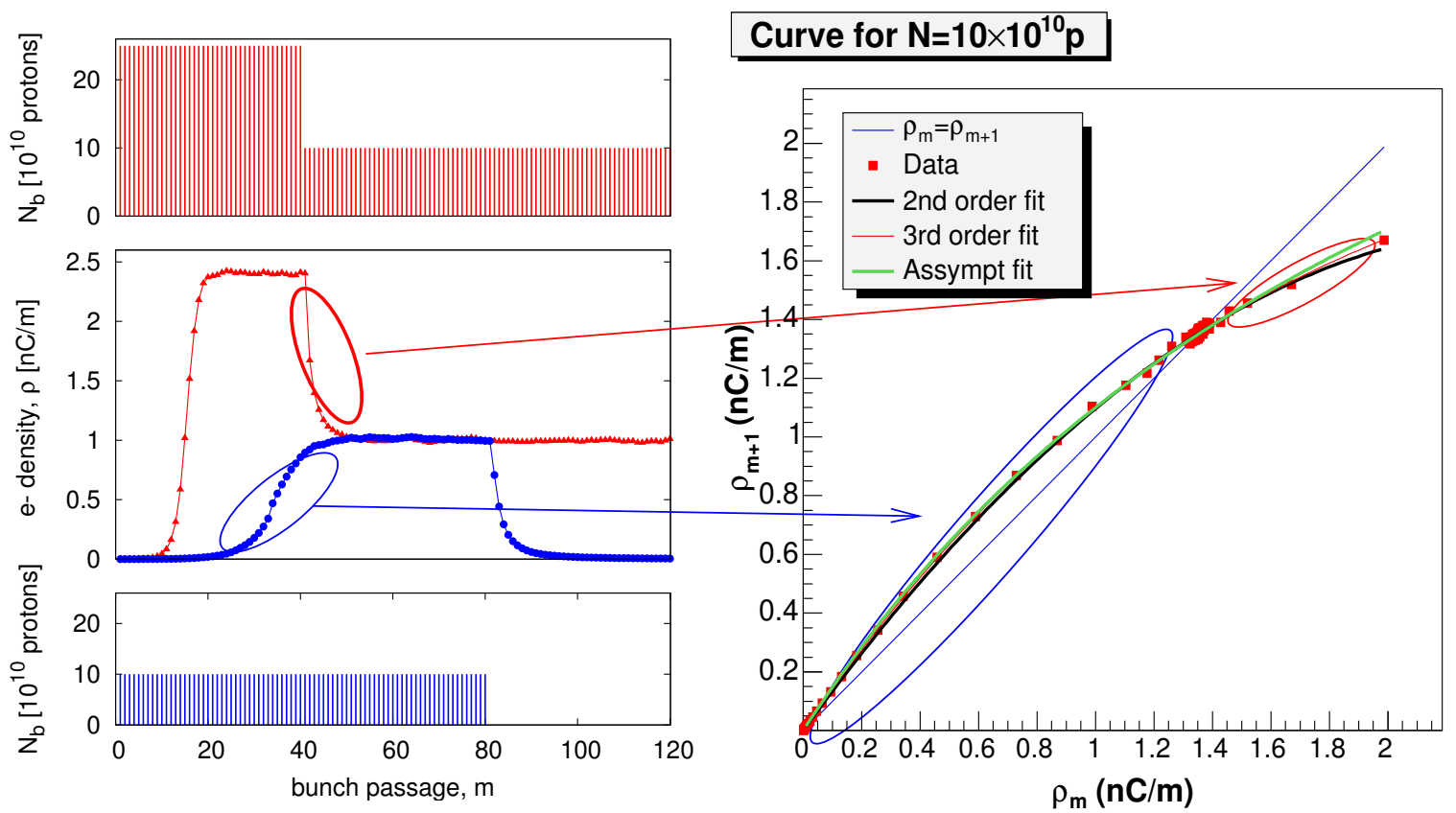

Figure 10.4: Left: Two different bunch trains (top and bottom plots) produce the same final saturated density (middle plot) when the final bunch intensity coincides. Right: Evolution in the $\left(\rho_{m}, \rho_{m+1}\right)$ space of the electron density merging the points with the same bunch intensity from the left hand side picture. All data points can be fitted using the same curve.

Table 10.2: List of input parameters for CSEC simulations, and for the calculations of the linear map coefficient, also called $\delta_{\text {eff }}$. The scan in bunch intensity $N$ is carried out in steps of $2 \times 10^{10}$ protons, the scan in $\delta_{\max }$ is carried out in steps of 0.2 .

\begin{tabular}{|c|c|c|c|}
\hline parameter & symbol & unit & value \\
\hline bunch spacing & $t_{s b}$ & ns & 108 \\
\hline full bunch length & $\sigma_{l}$ & ns & 15 \\
\hline bunch population & $N$ & $10^{10}$ protons & scan from $2-20$ \\
\hline rms beam radius & $\sigma_{r}$ & $\mathrm{~mm}$ & 2.0 \\
\hline pipe radius & $b$ & $\mathrm{~mm}$ & 60 \\
\hline maximum SEY & $\delta_{\max }$ & $\ldots$ & scan from $1.6-2.2$ \\
\hline reflection probability for $E \rightarrow 0$ & $\delta_{0}$ & $\ldots$ & 0.6 \\
\hline reflection probability for $E \rightarrow \infty$ & $\delta_{\infty}$ & $\ldots$ & 0.2 \\
\hline energy at maximum SEY & $E_{\max }$ & $\mathrm{eV}$ & 300 \\
\hline reflection energy & $E_{r}$ & $\mathrm{eV}$ & 60 \\
\hline energy of secondary emitted electrons & $E_{\mathrm{sec}}$ & $\mathrm{eV}$ & $8.9 / 5$ \\
\hline SEY exponent & $s$ & $\ldots$ & 1.83 \\
\hline
\end{tabular}



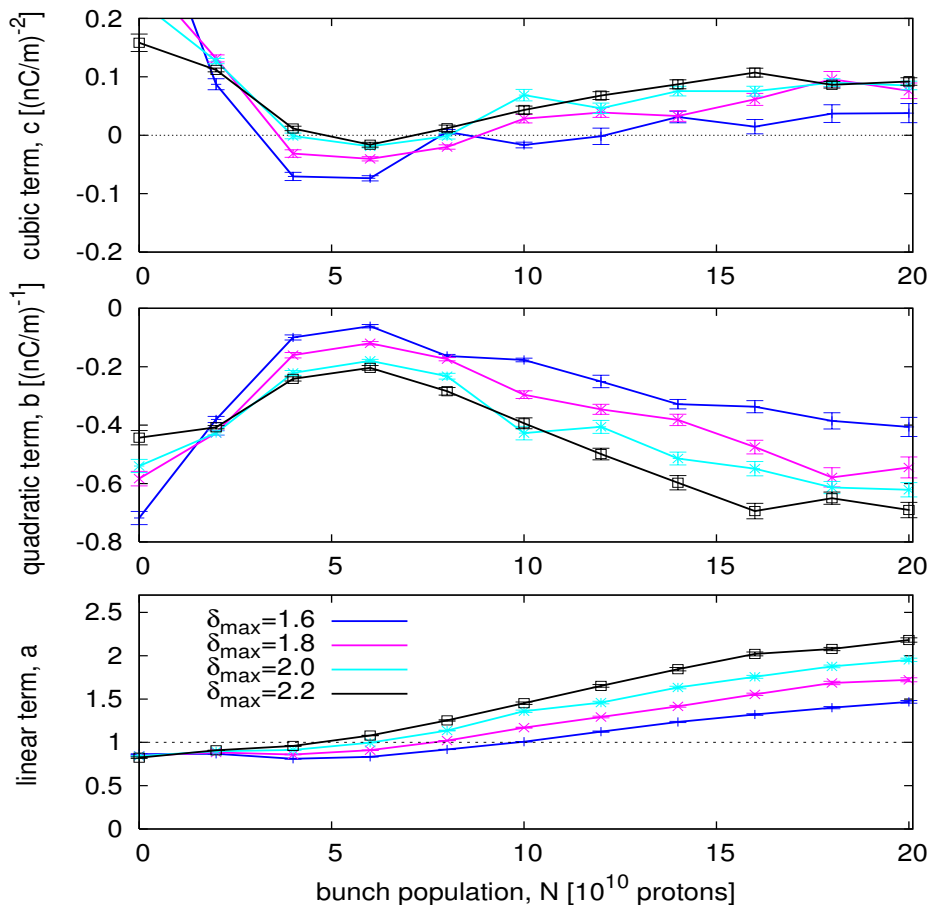

Figure 10.5: Evolution of the linear $a$, second order $b$, and third order $c$ terms of the cubic map as a function of the bunch intensity $N$ for different values of $\delta_{\max }$. The curves are obtained by fitting to CSEC simulations results.

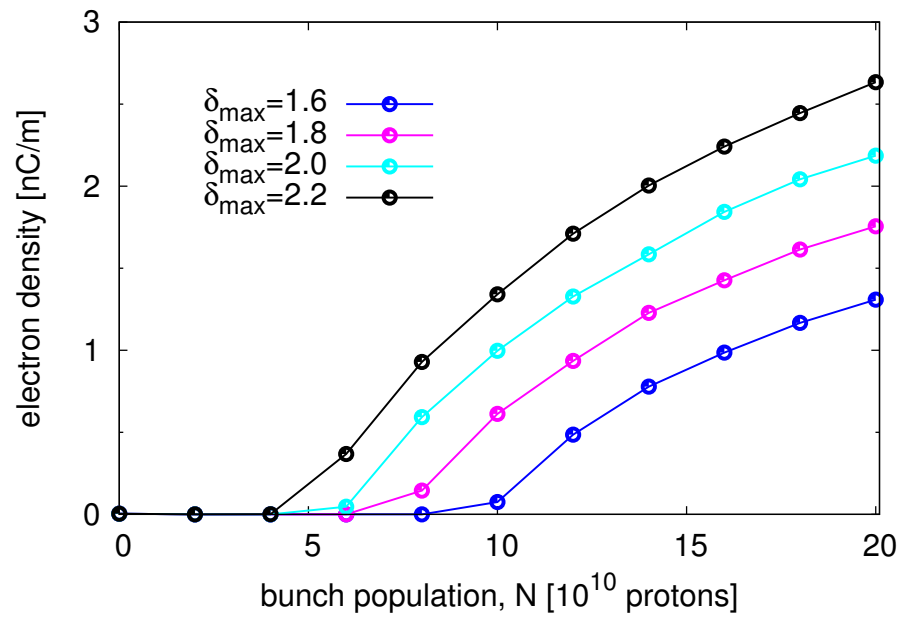

Figure 10.6: Peak electron densities as a function of the bunch intensity $N$ for the simulations in Fig. 10.5. Larger values of $\delta_{\max }$ decreases the electron cloud threshold, which consistently coincides with the bunch population at which $a=1$ in Fig. 10.5. 


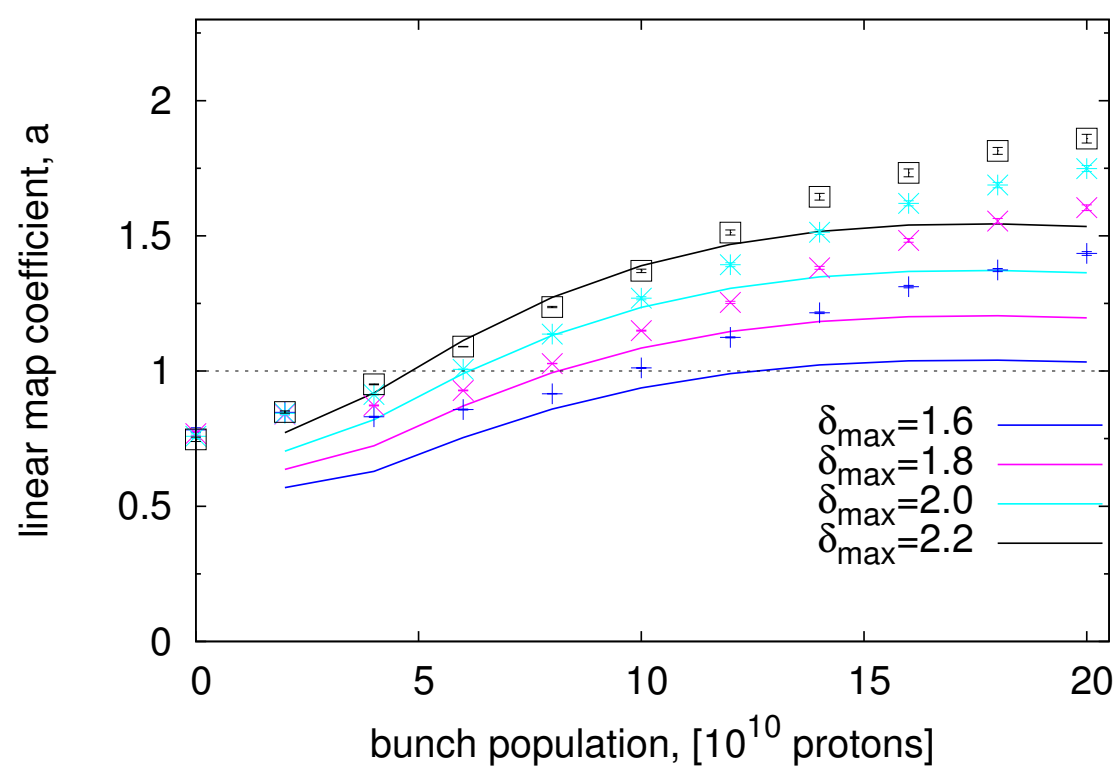

Figure 10.7: Comparison of the linear map coefficient $a$ as a function of the bunch population $N$ for different values of $\delta_{\max }$. The coefficients are derived from two ways: 1) using CSEC simulations (points), and 2) using the analysis in Section 10.1 (lines with the same color).

Figure 10.7 compares the result of the calculation of the linear map coefficient $a$ (also called the effective secondary emission yield, $\left.\delta_{\text {eff }}\right)$ using two methods and for different values of $\delta_{\max }$ : after fitting the CSEC simulation results (marks), and through the calculation following Eq. 10.32 (lines). The color of the marks and lines coincide for the same $\delta_{\max }$. Both results acceptably agree in the general evolution of the parameter $a$. Nonetheless, three regimes are distinguished:

1. From $N=0$ to $N \approx 5 \times 10^{10}$ protons/bunch.

The simulation results coincide independently of $\delta_{\max }$, indicating that in this range $a$ depends mainly on the behaviour of the low energy electrons. When using the analytical approach, smaller values of $a$ are found for smaller values of $\delta_{\max }$.

2. From $N \approx 5$ to $\approx 12 \times 10^{10}$ protons/bunch.

The agreement is reasonably good in this range, except perhaps for the case $\delta_{\max }=1.6$, for which the analytical approach results in slightly smaller values of $a$.

3. For $N>12 \times 10^{10}$ protons/bunch.

Above this limit, the average energy gain $E_{g}$ (due to the bunch passage) is larger than the energy at which the SEY has its maximum, $E_{\max }$. As explained previously, the contribution of the rediffused electrons is important (but not taken into account by the analytical approach). 


\section{Chapter 11}

\section{Maps for coupled electron and ion clouds}

Chapters 9 and 10 introduced the use of maps for reproducing the electron cloud in a bunchto-bunch evolution. A simple mathematical model is developed here to investigate possible explanations for sudden changes in multipacting-related outgassing rates, so called first-order phase transitions, regularly observed (and not explained) in RHIC [79]. Dynamical models of cloud build-up, and of the phase transition from "cloud off" to "cloud on", are enhanced when simple coupling between electron and ion clouds is included. Maps are then capable of reproducing the first order phase transitions seen in practice. They also predict that hysteresis, period doubling, and chaotic phenomena may be observed.

\subsection{Introduction}

A linear relation between the pressure and the electron flux was experimentally demonstrated in Chapter 5. Chapters 6 and 7 showed that computer simulation codes (like CSEC or ECLOUD) acceptably reproduce the experimental observations in the electron detector and pressure gauges. However, not all of the experimental observations related to the pressure behaviour in RHIC are fully understood. Next, two examples are described where RHIC observations are hardly explained with the present understanding of the electron cloud.

\subsubsection{A vacuum instability}

Figure 11.1 shows a pressure instability with a slow exponential growth (growth time, $\tau_{g} \approx$ $10 \mathrm{~s}$ ). This points towards the existence of a feedback mechanism in which the pressure is crucially involved. This instability is likely to be caused by rest gas ionization by the beam and the electron cloud, and it is described by [107]

$$
P=\frac{Q_{0}+\eta_{e} k T \frac{L}{e} \frac{d I}{d l}}{S-\eta_{\text {ion }} L b_{0}}
$$




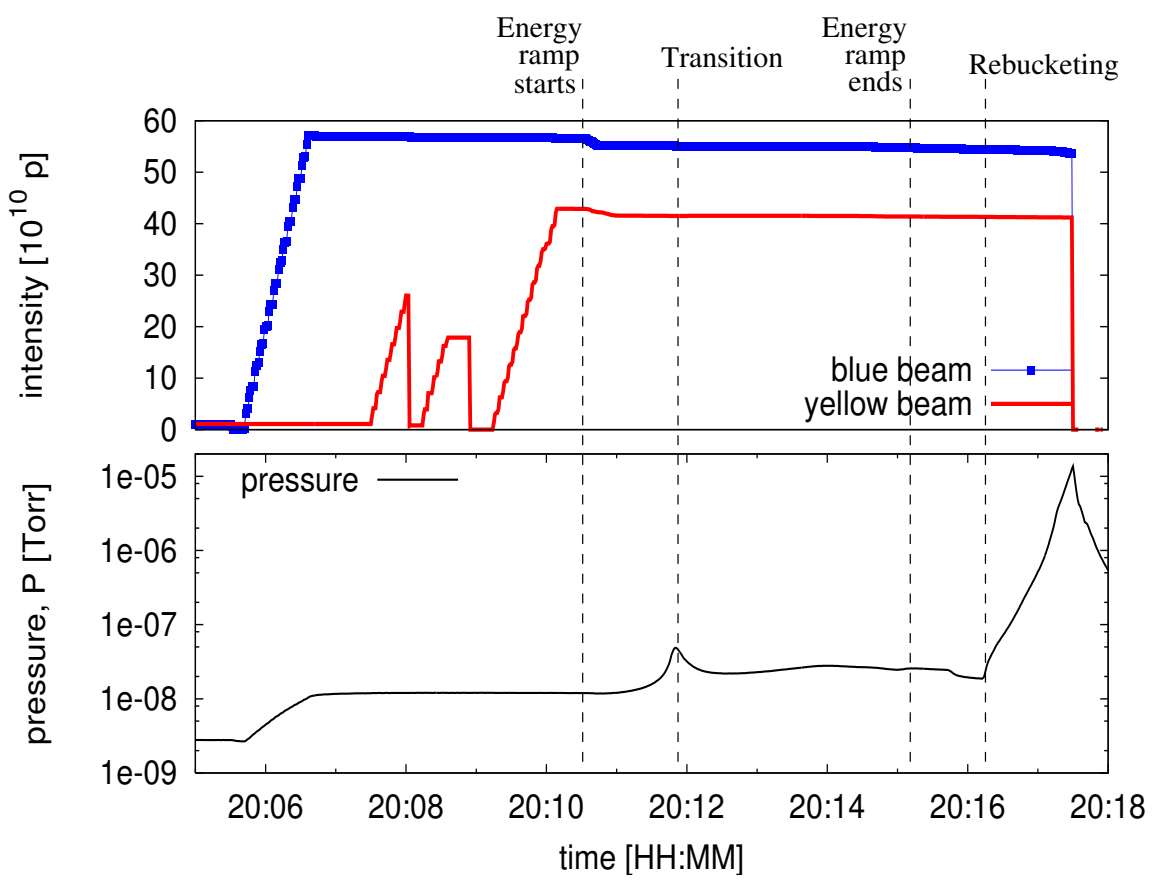

Figure 11.1: A vacuum instability with gold beams in RHIC [107]. The upper plot shows the evolution of the beam intensity for both rings during injection, acceleration, and storage. The bottom plot shows the pressure instability that occurred in the collimator region of the blue ring.

where $Q_{0}$ is the static gas load, $\eta_{e}$ is the electron induced molecular desorption coefficient, $k$ is the Boltzmann's constant, $T$ is the temperature, $e$ is the absolute electron charge, $2 L$ is the distance between two consecutive vacuum pumps, $d I / d l$ is the linear electron flux to the wall, $2 S$ is the pumping speed, and $\eta_{\text {ion }}$ is the ion induced molecular desorption coefficient. The parameter $b_{0}$ is defined as [107]

$$
b_{0}=\sigma_{e} \frac{2 R_{p}}{e} \frac{d I_{e}}{d l}+\sigma_{b} \frac{I_{b}}{e},
$$

where $\sigma_{e}$ is the cross section for rest gas ionization from an impact of cloud electrons, $R_{p}$ is the beam pipe radius, $\sigma_{b}$ is the cross section for rest gas ionization by the beam, and $I_{b}$ is the beam current.

In order to obtain similar growth rates, the ion desorption coefficient $\eta_{\text {ion }}$ in Eq. 11.1 needs to be between one or two order of magnitudes larger than those described by the literature: while the literature in the field reports $\eta_{\mathrm{CO}}=0.3$ molecules/CO-ion [66], Ref. [107] calculates $\eta_{\mathrm{CO}}=2.8$ molecules/CO-ion.

\subsubsection{First and second order phase transitions in RHIC}

Experimental data shown in Fig. 11.2 illustrate how both first and second order phase transitions are seen in RHIC, as a threshold bunch population is crossed. Figure 11.2 plots 
pressure versus average bunch population for two gauges in IR10, and two more gauges in IR12. While the pressure in IR12 smoothly decreases as the bunch population threshold is crossed, in IR10 an abrupt transition is seen. The beam pipe is Beryllium in IR10, while is stainless steel in IR12 (see Fig. C.1 in Appendix C).

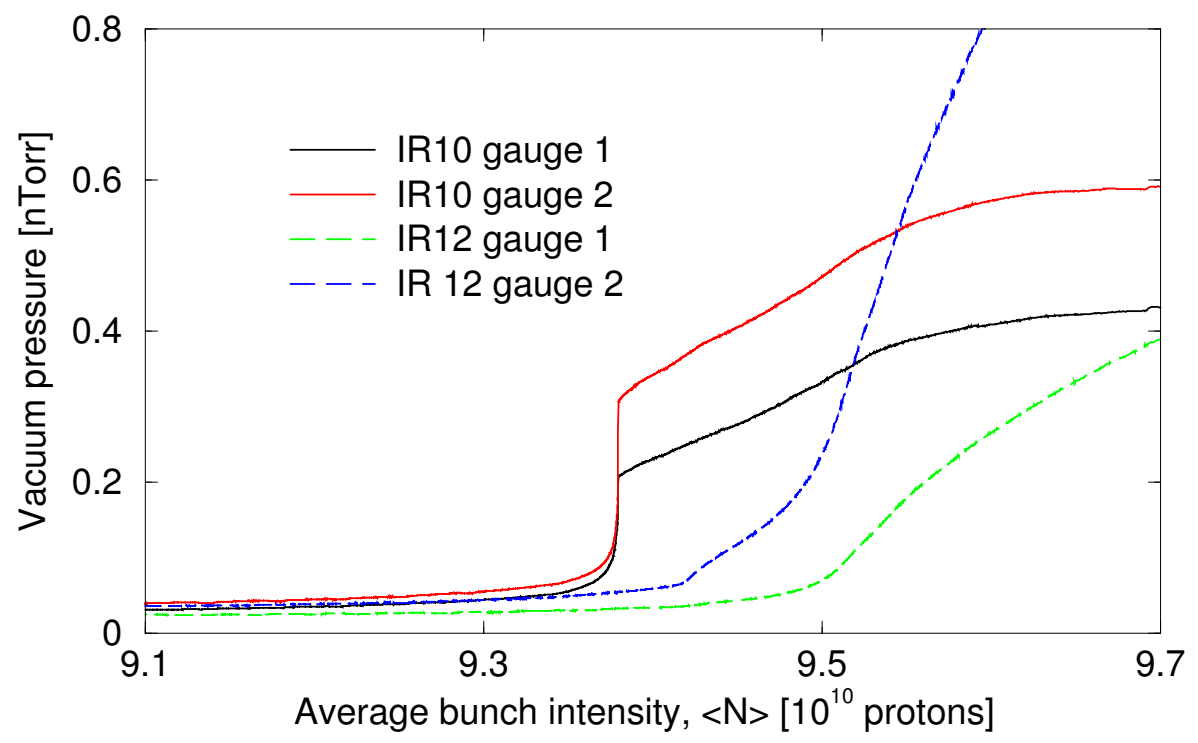

Figure 11.2: First and second order electron cloud phase transitions observed in IR10 and IR12. The data were taken as the bunch population slowly decayed during beam fill 5905 . The pairs of gauges in IR10 and IR12 are about $15 \mathrm{~m}$ and $1 \mathrm{~m}$ apart, respectively. The actual copper ion bunch population is converted to an equivalent average number of protons per bunch.

Since the pressure is proportional to the average electron flux over one turn, $\langle d I / d l\rangle_{\tau}$ (recall Chapter 5), it is sensible to expect that a jump in pressure is due to a jump in the electron density, i.e. a first order phase transition in the pressure indicates a first order phase transition in the electron density. In contrast, Fig. 10.6 shows that independent of $\delta_{\max }$, the phase transition of the saturated electron density as a function of the bunch population is second order.

\subsection{Phase transitions using Maps for Electron Clouds}

Using bunch-to-bunch maps in Chapter 9, a saturated electron density is found when

$$
\rho_{m+1}=\rho_{m} \equiv \rho^{*},
$$

and for the parabolic map in Chapter 9 (Eq. 9.1), it follows that

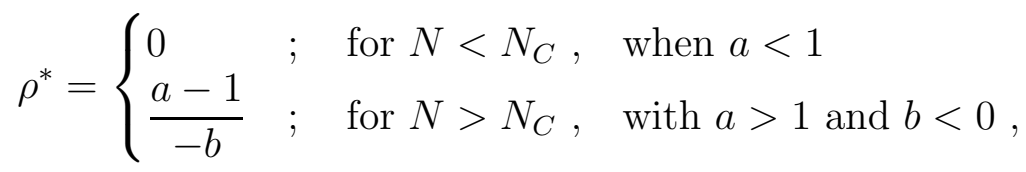


where $N_{C}$ is the critical bunch intensity at which the transition (from electron cloud "off" to "on") occurs. The coefficient $a$ increases monotonically with the bunch population $N$ for a fixed set of beam pipe parameters, - see the results from both CSEC simulations and the analytical approach in Fig. 10.7. In this case, the stable electron cloud density $\rho^{*}(N)$ is also a function of bunch population. Equation 11.4 then predicts that the phase transition is second order $-\rho^{*}(N)$ increases smoothly from zero above a critical threshold population, when $a>1$. This is also seen in Fig. 10.6 and Ref. [79].

The catastrophic collapse of the pressure routinely observed in IR10 represents an unexpected behaviour. A sudden drop in the pressure is difficult to understand since the surface parameters show a smooth dependence on the impact electron energy at the wall (see Section 3.3 and References therein). Thus, the observation of both first and second order phase transitions in RHIC suggests that additional physics should be taken into account.

\subsection{Ion clouds}

A candidate for additional physics is the interplay between electron clouds and the positive ion clouds present when enhanced pressures are produced inside the vacuum chamber (this is in part motivated by the vacuum pressure instability seen in Fig. 11.1). An early analysis for the Large Hadron Collider in Ref. [108] concluded that this interplay would not significantly affect accelerator performance. Nonetheless, there may be circumstances when such effects become significant, especially for heavy ions. In particular, it is re-introduced to explain experimental observations at RHIC (see Eq. 11.1 and Ref. [107]). These effects have resurfaced more recently as a future concern again for the LHC [109].

Models of this interplay face two main challenges: first, a significant number of uncertain surface physics parameters for both electron and ions. Apart from the usual SEY parameters for the electron cloud build up, Eqs. 11.1 and 11.2 involve ionization cross-sections, and electron and ion desorption coefficients, which are not only a function of the impacting particle energy, but also of the gas species. Secondly, the time scales for electron and ion cloud dynamics are extremely different. Long ion lifetimes imply very long CPU times for simulations. Not only is the typical time-of-flight between surfaces much longer for a massive ion than for an electron of similar kinetic energy, but also the backscattering probability for ion energies below about $30 \mathrm{eV}$ is close to unity [45]. The lifetime of such ions is not characterized by their time-of-flight, as with electrons, but by vacuum pumping times, usually measured in seconds. Therefore, electron clouds evolve with a lifetime on the order of 1 microsecond, while ion clouds evolve between three and six orders of magnitude more slowly.

Thus, achieving a detailed understanding of such complex phenomena is a challenging problem to say the least. Next, maps are used to circumvent these challenges and give an improved intuitive understanding of the evolution of electron clouds in conjunction with ion clouds and show how, at least in principle, first order phase transitions may come about. 


\subsection{Coupled maps and fixed points}

In a map model, the interplay between electron clouds and ion clouds is generally expressed by

$$
\begin{aligned}
\rho_{m+1} & =f\left(\rho_{m}, R_{m}\right) \\
R_{m+1} & =g\left(\rho_{m}, R_{m}\right),
\end{aligned}
$$

where $R_{m}[\mathrm{nC} / \mathrm{m}]$ is the ion cloud density after the passage of the $m$ 'th bunch. (Both $\rho$ and $R$ are defined to be positive.) In the following, we use the vector $\vec{r}$ for the electron and ion densities

$$
\vec{r}_{m}=\left(\begin{array}{c}
\rho_{m} \\
R_{m}
\end{array}\right) \text {. }
$$

A fixed point is found when

$$
\vec{r}_{\mathrm{m}+1}=\vec{r}_{m} \equiv \vec{r}^{*}
$$

Furthermore, we need the fixed point to be stable. That is, small perturbations around the fixed point $\vec{r}^{*}$ must result in an evolution that converges towards the fixed point.

\subsubsection{Stability condition}

Close to a fixed point $\vec{r}^{*}$, the linear motion from bunch $m$ to $m+1$ is

$$
\vec{r}_{\mathrm{m}+1}=J \vec{r}_{m}
$$

where $J$ is the $2 \mathrm{x} 2$ Jacobian matrix

$$
J=\left(\begin{array}{cc}
\frac{\partial f}{\partial \rho_{m}} & \frac{\partial f}{\partial R_{m}} \\
\frac{\partial g}{\partial \rho_{m}} & \frac{\partial g}{\partial R_{m}}
\end{array}\right)_{\vec{r}^{*}},
$$

which determines the stability of the fixed point through its trace and determinant. Appendix $\mathrm{D}$ shows that a fixed point is stable if one of these two conditions is fulfilled:

$$
\begin{aligned}
\text { i) } t^{2}<d^{2} & \text {; and } d^{2}<1 \\
\text { ii) } t^{2}>d^{2} & \text {; and }|t|+\sqrt{t^{2}-d^{2}}<1
\end{aligned}
$$

where the convenient definitions

$$
\begin{aligned}
t & \equiv \operatorname{Tr}\left(J^{2}\right) / 2 \\
d & \equiv \operatorname{det}(J)
\end{aligned}
$$

have been introduced. If neither of these conditions applies, then the motion diverges. Appendix D shows, moreover, that two different motions can be obtained around the fixed points: elliptical or hyperbolic. Figure 11.3 shows the stability regions in $(t, d)$ space.

Note that the stability conditions developed here do not depend on the form of the functions $f$ and $g$ (Eqs. 11.5 and 11.6). That is to say, these conditions are valid for any coupling mechanism expressed by $f$ and $g$. 


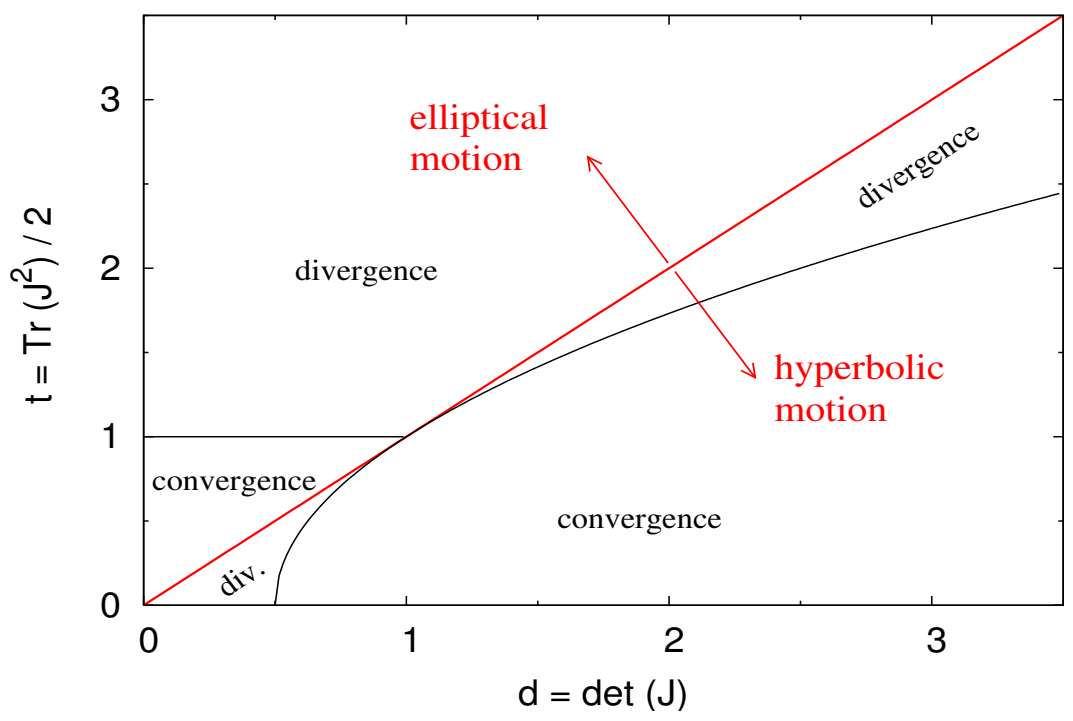

Figure 11.3: Stability regions as a function of the parameters $t$ and $d$ (Eqs. 11.13 and 11.14), obtained from the Jacobian matrix. Appendix D shows that above the $45^{\circ}$ line the motion shows elliptical behaviour, while below the $45^{\circ}$ line the motion shows a hyperbolic behaviour.

\subsection{A simple coupled maps model}

In order to visualize the phenomena that these conditions can generate, take an example for the functions $f$ and $g$ based on the cubic map for the electron density (Eq. 9.5). Consider the "proof-of-principle" coupled maps

$$
\begin{aligned}
\rho_{m+1} & =\left(a+b \rho_{m}+y R_{m}\right) \rho_{m}+c \rho_{m}^{3} \\
R_{m+1} & =A R_{m}+Y \rho_{m} .
\end{aligned}
$$

If the coupling coefficients are turned off $(y=Y=0)$, then the electron cloud map Eq. 9.5 is recovered, along with the uncoupled ion map

$$
R_{m+1}=A R_{m}
$$

Values of $A$ smaller than unity correspond to ion cloud clearance, for example through vacuum pumping and neutralization. Since the massive ion clouds only clear slowly, it is expected that $A \approx 1$, where $A>1$ marks the threshold for an unlimited ion cloud growth (ion instability).

There are two coupling mechanisms in Eqs. 11.15 and 11.16:

1. First, electrons generate a positive ion cloud, for example by colliding with the rest gas in the vacuum chamber. This is represented by the term $Y \rho_{m}$. $Y$ is positive, but its order of magnitude is not trivially apparent. 
2. Second, the slow moving positive ions tend to neutralize the negative electron space charge of the accumulated electron cloud. This is represented by the term $y R_{m}$. Physical values of $y$ are positive, and of the same magnitude as $b$, so $y \sim|b|$.

In this example, the electron cloud densities at the fixed points correspond to the roots of the cubic equation

$$
\rho^{*}=a \rho^{*}+b^{\prime} \rho^{* 2}+c \rho^{* 3}
$$

where the "effective space charge coefficient"

$$
b^{\prime}=b-(y Y /(A-1))
$$

has been conveniently introduced. One of the 3 roots $\left(\rho^{*}=0\right)$ is trivial. The 2 nd and $3 \mathrm{rd}$ roots,

$$
\rho^{*}=\frac{-b^{\prime} \pm \sqrt{{b^{\prime}}^{2}-4 c(a-1)}}{2 c}
$$

are only physical if their values are real and positive.

Finally, the stationary ion density is simply related to the stationary electron density by

$$
R^{*}=-\frac{Y}{A-1} \rho^{*},
$$

(recall that $Y$ is positive and $(A-1)$ is negative).

\subsubsection{Numerical application}

Next, we assume that all of the coupled map coefficients are constants except for the weak electron cloud growth rate $a$, which is presumed to depend linearly on the bunch population according to

$$
a=0.4+0.1\left(N / 10^{10}\right) .
$$

The coupled map coefficients used throughout below and quoted in Table 11.1 are illustrative. (They are not intended to quantitatively reproduce RHIC results.)

Table 11.1: Map parameters used in the following examples.

\begin{tabular}{|c|c|c|c|c|c|}
\hline \hline$a$ & $b$ & $c$ & $y$ & $A$ & $Y$ \\
\hline Eq. 11.22 & -0.1 & -0.08 & 0.4 & 0.96 & 0.03 \\
\hline \hline
\end{tabular}

The right hand side of Eq. 11.18 is plotted in Fig. 11.4 for three bunch populations. The fixed point solutions are found where these curves intersect with the left hand side of Eq. 11.18 - that is, the identity map or the $45^{\circ}$ line. Figure 11.4 shows that three fixed electron densities exist for $N=5 \times 10^{10}$ protons/bunch: $\rho_{1}^{*}=0 \mathrm{nC} / \mathrm{m}, \rho_{2}^{*}=0.69 \mathrm{nC} / \mathrm{m}$ and $\rho_{3}^{*}=1.81 \mathrm{nC} / \mathrm{m}$. Calculating the Jacobian matrix at the three solutions, their corresponding stability is obtained using Eqs. 11.11 and 11.12: 


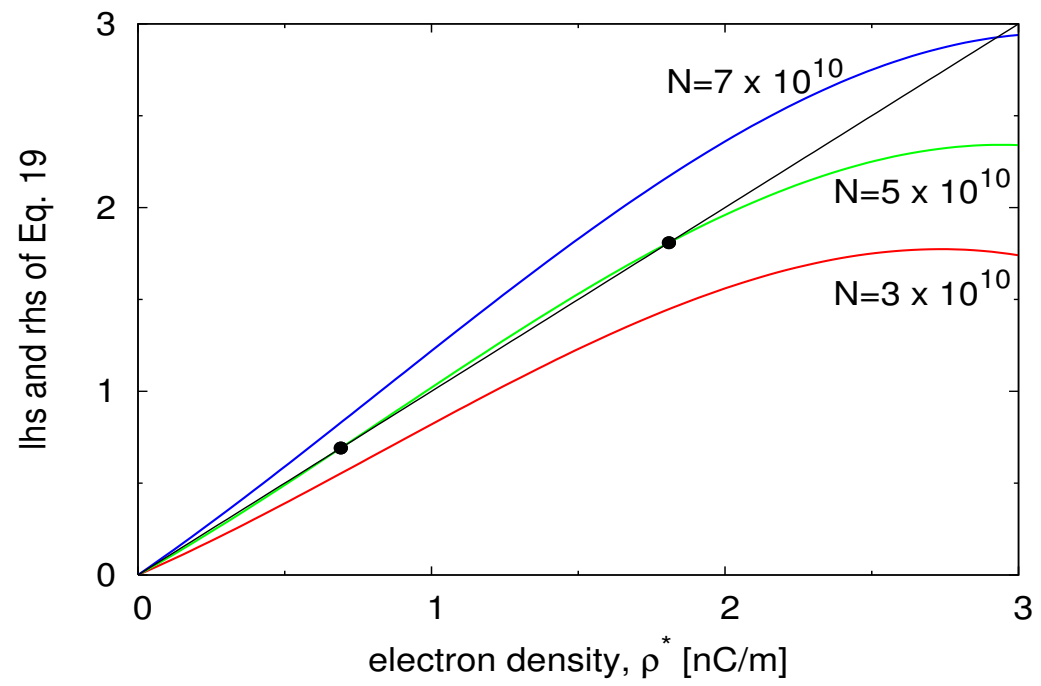

Figure 11.4: Three different representations of the right hand side (rhs) of Eq. 11.18 corresponding to $N=3,5$ and $N=7 \times 10^{10}$ protons/bunch. The straight line shows the left hand side (lhs) of Eq. 11.18. Intersections between the rhs and lhs of Eq. 11.18 mark the stationary electron densities. Two non-zero solutions exist for $N=5 \times 10^{10}$ protons per bunch: at $\rho^{*}=0.69$ and $\rho^{*}=1.81 \mathrm{nC} / \mathrm{m}$. Their stability is examined in Fig. 11.5.
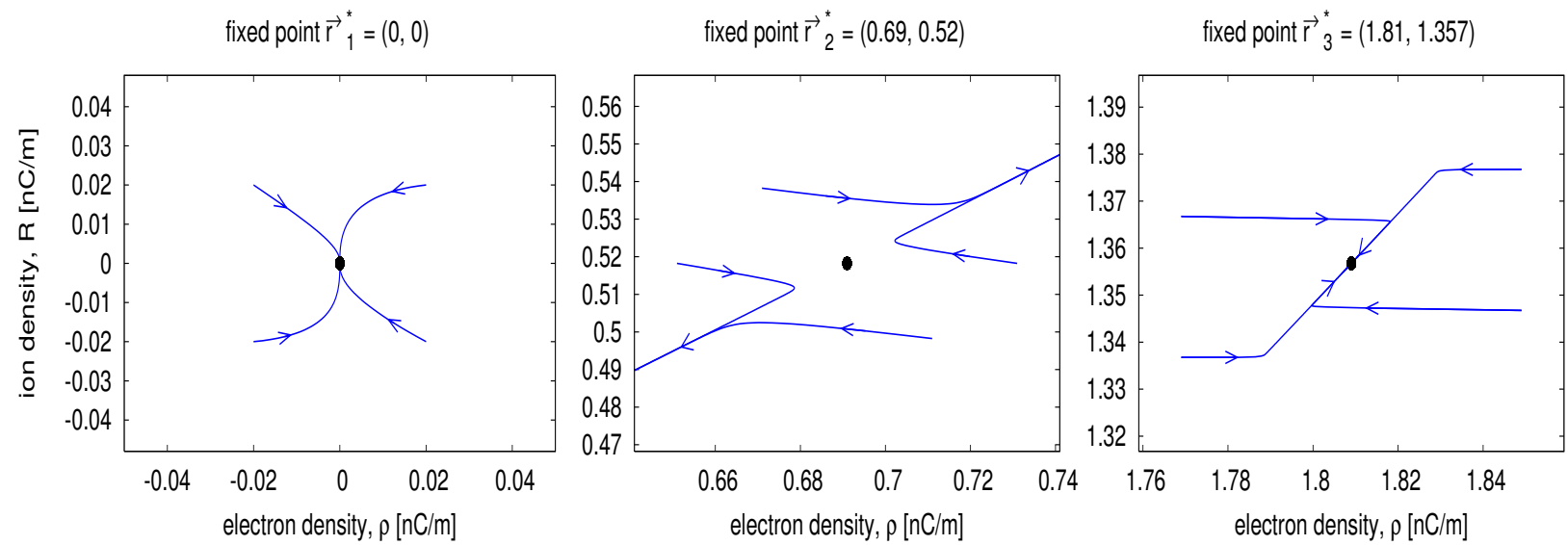

Figure 11.5: Stability around the 3 fixed points (marked with a bold point) for $N=5 \times 10^{10}$ protons/bunch. The first solution (left) shows an elliptical converging motion. The second solution (middle plot) shows a hyperbolic diverging motion, through which small perturbations around the fixed point $\vec{r}_{2}^{*}$ diverge quickly towards $\vec{r}_{1}^{*}$ or $\vec{r}_{3}^{*}$, depending on the initial conditions. The third solution (right) shows a hyperbolic converging motion that attracts all the points in its vicinity. Recall that only trajectories with positive $\rho, R$ are physically sensible; the motion depicted around $\vec{r}_{1}^{*}$ includes negative values of $\rho, R$ just as a numerical example. Fixed points $\vec{r}_{1}^{*}$ and $\vec{r}_{3}^{*}$ are attractors, while $\vec{r}_{2}^{*}$ is a repeller. 
1. the first fixed point $\vec{r}_{1}^{*}=(0,0)$ shows (see left plot of Fig. 11.5):

$$
t^{2}<d^{2} \rightarrow \text { elliptic motion ; } \quad d^{2}<1 \rightarrow \text { convergence }
$$

2. the second fixed point $\vec{r}_{2}^{*}=(0.69,0.52)$ shows (see left plot of Fig. 11.5):

$$
t^{2}>d^{2} \rightarrow \text { hyperbolic motion ; }|t|+\sqrt{t^{2}-d^{2}}>1 \rightarrow \text { divergence }
$$

3. the third fixed point $\vec{r}_{2}^{*}=(1.81,1.357)$ shows (see right plot of Fig. 11.5):

$$
t^{2}>d^{2} \rightarrow \text { hyperbolic motion ; }|t|+\sqrt{t^{2}-d^{2}}<1 \rightarrow \text { convergence }
$$

Note that for $N=5 \times 10^{10}$ protons/bunch, Eq. 11.22 sets $a=0.9<1$, which, according to the first order uncoupled map (Eq. 10.1 or Eq. 11.4), this does not produce any electron cloud (stable or unstable). However, the presence of a coupled ion cloud enhances the electron survival, and thus electron clouds are created even when $a<1$. Another crucial effect of the coupling is to change the sign of the effective space charge coefficient $b^{\prime}$ - positive ions neutralize the negative space charge of the electrons at intermediate bunch populations, permitting different physical stationary solutions.

\subsection{First order phase transitions and hysteresis}

These conditions lead to a first order phase transition, and to hysteresis. Figure 11.6 shows the results of a dynamical simulation, in which the coupled maps are applied directly, first as the bunch population is slowly decreased, and then as it is slowly increased. The solid line shows that the stable electron cloud density decreases as the bunch population is reduced, until at $N \approx 4.7 \times 10^{10}$ the electron cloud collapses catastrophically. When the bunch population is then slowly increased, no electron (or ion) cloud forms up to a population of $N=6.0 \times 10^{10}$, when the cloud grows rapidly to a stable stationary value.

Figure 11.7 shows the flow in $(\rho, R)$ space for different bunch populations: $N=3,5$ and $7 \times 10^{10}$ protons/bunch. These plots result from tracking several simulations with different initial conditions. For $N=3 \times 10^{10}$ (left plot), all trajectories are attracted to the global attractor at the $\vec{r}^{*}=(0,0)$ fixed point. Similar behaviour is found for $N=7 \times 10^{10}$ (right plot), where all trajectories converge to the global attractor at $\vec{r}^{*}=(2.9,2.17)$, no matter what initial conditions are used. Note that there is also a fixed point global repeller at $\vec{r}^{*}=(0,0)$.

However, the situation is different for $N=5 \times 10^{10}$ protons/bunch (middle plot in Fig. 11.7). Two different basins of attraction coexist: one corresponding to the fixed point $\vec{r}^{*}=(0,0)$, the second corresponding to the point $\vec{r}^{*}=(1.81,1.357)$. This feature is the origin of the hysteresis and the first order phase transitions. The boundary between the two basins (dashed line, middle plot in Fig. 11.7) moves to the upper right of the plot as the bunch population smoothly decreases below $N=5 \times 10^{10}$, until the two non-zero fixed points coincide at about $N=4.7 \times 10^{10}$ protons/bunch. At this point the second basin disappears, and all trajectories collapse to $\vec{r}^{*}=(0,0)$, no matter what their initial conditions. 


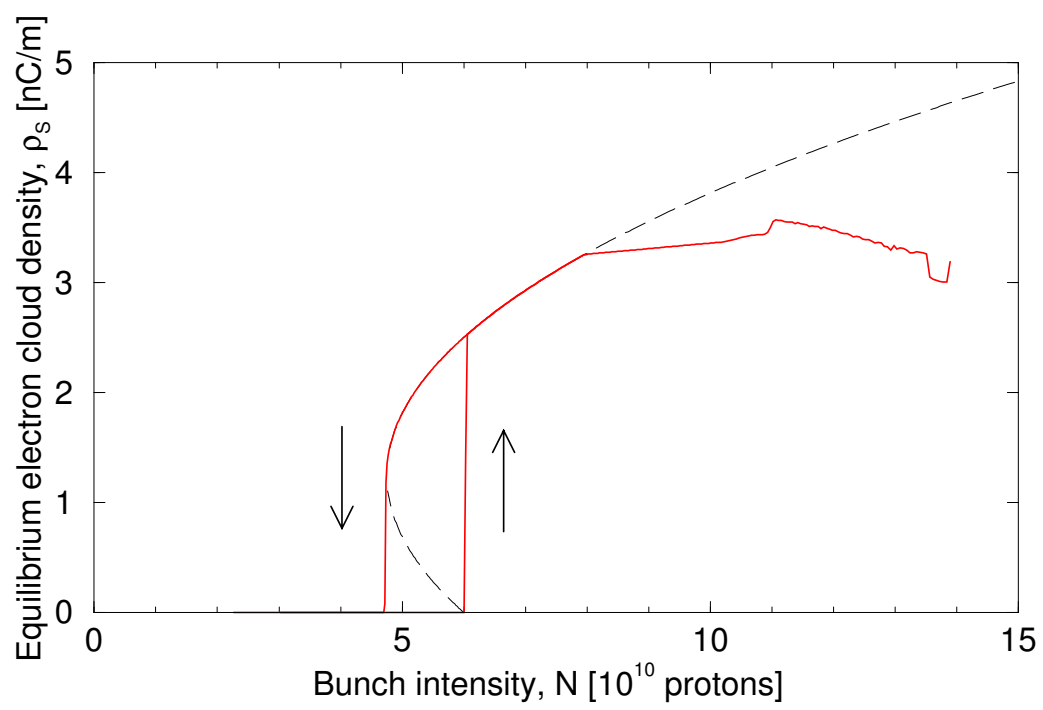

Figure 11.6: Evolution of the electron cloud density as the bunch population $N$ is first slowly decreased, and then slowly increased. The precipitous and hysteretic behavior is typical of first order phase transitions. The dashed lines represent the two stationary solutions described in Eq. 11.20.
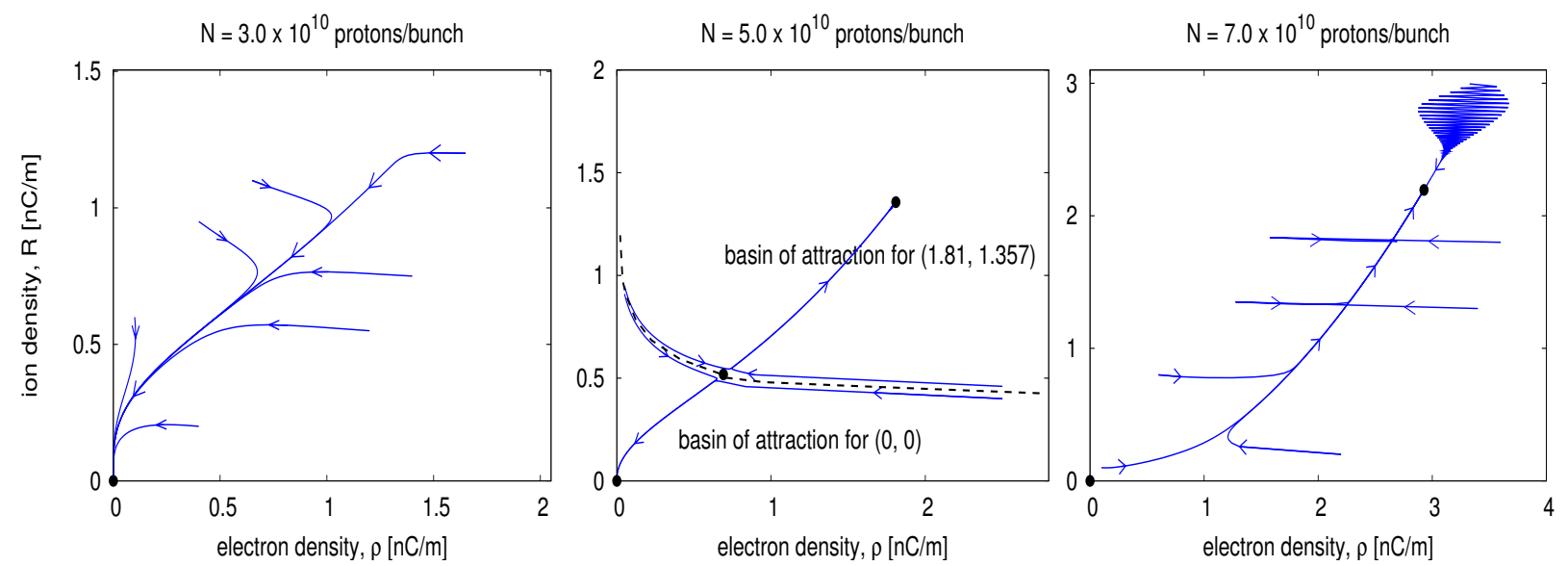

Figure 11.7: Coupled motion in $(\rho, R)$ space is tracked for $N=3 \times 10^{10}$ (left plot), $N=5 \times 10^{10}$ (middle plot), and $N=7 \times 10^{10}$ protons/bunch (right plot). Only one fixed point $-\vec{r}^{*}=(0,0)-$ exists for $N=3 \times 10^{10}$, which acts as a global attractor. There are two basins of attraction for $N=5 \times 10^{10}$ protons/bunch: one containing the fixed point $\vec{r}_{1}^{*}=(0,0)$, and the second containing $\vec{r}_{3}^{*}=(1.81,1.357)$. The fixed point $\vec{r}_{2}^{*}=(0.69,0.52)$ sits on the boundary between the two basins, acting as a global repeller. Thus, the system evolve towards $\vec{r}_{1}^{*}$ or $\vec{r}_{3}^{*}$, depending on the initial conditions. The fixed point $\vec{r}^{*}=(2.9,2.17)$ is a global attractor for $N=7 \times 10^{10}$ protons/bunch, and all trajectories converge to it as $m \rightarrow \infty$. In this case the fixed point $(0,0)$ is a repeller. 

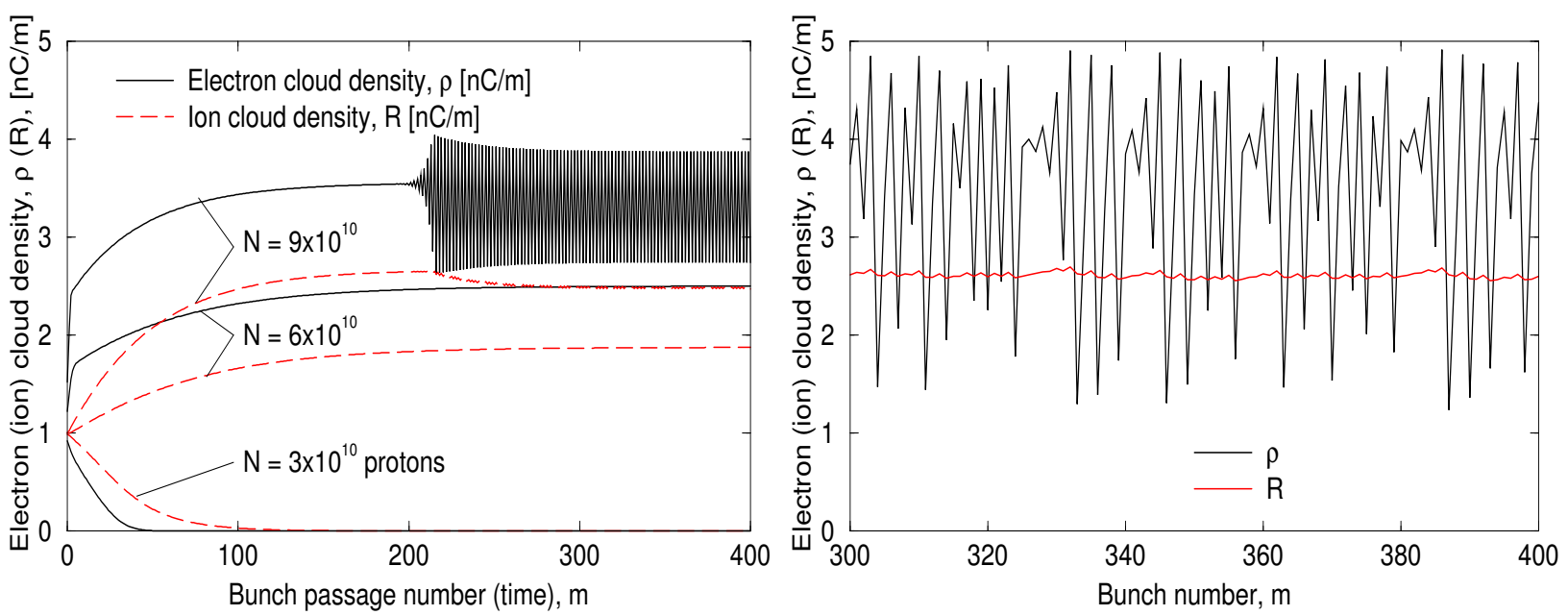

Figure 11.8: The left hand side plot shows the dynamical evolution of the electron and ion cloud densities as a function of time (bunch passage number) for 3 different bunch intensities, $N=3 \times 10^{10}, 6 \times 10^{10}$, and $9 \times 10^{10}$ protons per bunch. The right hand side depicts a zoom between the bunch passages 300 and 400 to show the chaotic behavior of electron and ion cloud densities when $N=12 \times 10^{10}$ protons per bunch.

\subsection{Additional dynamical phases}

Figure 11.8 (left) shows the evolution of the electron and ion clouds for different bunch populations, always starting with the same (arbitrary) initial cloud densities. The clouds decay away or build to stable solutions with $N=3 \times 10^{10}$ and $6 \times 10^{10}$, consistent with the usual behaviour of uncoupled electron clouds. However, the clouds evolve into a stable period-2 oscillation when $N=9 \times 10^{10}$ protons/bunch. Figure 11.8 (right) takes a closer look at the chaotic dynamics that evolve when $N=12 \times 10^{10}$. In this case, coupled maps enhance the generation of period doubling and chaos. Such additional dynamical phases have not (yet) been observed in electron clouds in accelerators, but it is possible they occur at, or near, typical operating conditions.

\subsection{Discussion}

The "proof-of-principle" coupled maps presented here can generate electron and ion clouds that turn "on" and "off" precipitously. Such first order phase transitions are sometimes seen (through its surrogate, the vacuum pressure) in practice, but up to date have been not reproduced by the contemporary simulation codes that model electron clouds in isolation. Other coupling mechanisms than those presented here are also plausible, and can be modeled in the map formalism. For instance, Ref. [110] presents a similar mechanism in which the electron survival is enhanced by the presence of positive ions, thus enlarging the linear map 
coefficient $a$. The dependence of $a$ on the electron density brings up hysteresis and first order phase transitions. Both descriptions are just schematic and should not be taken too literally. A detailed modeling of such complicated dependencies will require better knowledge of relevant ion and electron desorption coefficients, ionization cross-sections, and non trivial low density plasmas, which are beyond the scope of this work. 


\section{PART IV}

\section{CONCLUSIONS}





\section{Chapter 12}

\section{Conclusions}

In this thesis, electron multipacting in RHIC has been studied by means of computer simulation codes, experimental observations, and theoretical analysis. An electron detector has been successfully installed in RHIC and is routinely operated to monitor electron multipacting activity in the vacuum chamber. With this detector, the pressure rises that occurred at RHIC after its first year of operation have been unambiguously linked to electron clouds. It has been shown that the pressure is proportional to the electron flux received by the chamber wall. Using this linear relation, the electron desorption coefficient from baked and unbaked stainless steel surfaces have been inferred, and found to be in agreement with measurements from the literature. This coefficient strongly depends on the energy at which the electron strikes the chamber surface. For unbaked stainless steel, a measurement of the energy spectrum during multipacting conditions was performed using the electron detector. The spectrum shows a large peak of low energy electrons around $10 \mathrm{eV}$, and it extends up to about $300 \mathrm{eV}$. Neither the lower nor the upper energy limit could be precisely determined because of electron detector noise. The spectrum was found to be in good agreement with the results using the computer simulation code CSEC.

Analytical formulae were found that describe the pressure rise in the presence of an electron cloud as the beam bunches are injected into the RHIC ring. It allows electron clouds to be recognized in the sections of the ring without electron detectors. Typically, the pattern is characterized by an initial exponential growth that continues until about the time that the bunch at which cloud saturation occurs is injected, then the growth enters a linear regime. The peak electron flux into the wall and the decay time have been experimentally measured using the electron detector, and compared with the values calculated using CSEC simulations. The CSEC simulations have been performed with different input files that scan two of the most significant Secondary Electron Yield (SEY) parameters: the maximum SEY, $\delta_{\max }$, and the SEY for zero electron energy, $\delta_{0}$. A set of values for $\left(\delta_{0}, \delta_{\max }\right)$ are obtained that reproduce the experimental observations. This set of values is in agreement with the surface physics literature.

The pressure rise in the interaction regions has been studied in this thesis. After an electron cloud formation due to the presence of two beams, the cloud decays in presence of only one beam. This decay time has been experimentally measured to be almost a factor 
of three larger than the decay in the single beam pipes (i.e., in absence of any beam). Reducing the surface SEY through coated NEG beam pipes (of about $1 \mu m$ thickness) has been demonstrated to be the most efficient method to counteract the electron multipacting in RHIC. The use of solenoids keeps the electrons around the beam pipe wall and decreases the electron energy gain, which reduces the electron multiplication. Consequently, the pressure when the solenoids are used is lower than it would be otherwise. However, the pressure rise is not fully suppressed and a residual effect is still visible.

A new approach to electron cloud build up is also presented. This approach models the electron density using a bunch-to-bunch technique by means of simple nonlinear maps of the form $\rho_{\mathrm{m}+1}=f\left(\rho_{m}\right)$, where $\rho_{m}$ is the electron density at bunch passage $m$. The form of the function $f\left(\rho_{m}\right)$ depends on coefficients, whose values are obtained by empirical fits to detailed electron cloud simulation codes, namely CSEC or ECLOUD. Using a third order polynomial map, a heuristic computer code is developed, MEC, which successfully reproduces the electron cloud density with different bunch patterns in a bunch-to-bunch approximation. The computation time using $\mathrm{MEC}$ is reduced by about 7 orders of magnitude with respect to the CSEC computation time. The reproducibility of the CSEC results using MEC implies an underlying simplicity in the electron cloud dynamics. Using the MEC maps, the actual polynomial values for the straight sections in RHIC demonstrate that the most sparse distribution of bunches is the most favorable against electron cloud formation. This "rule of thumb" has been routinely applied during RHIC operation to minimize electron multipacting cases, thereby maximizing its luminosity.

To first order, the function $f\left(\rho_{m}\right)$ is linearized to $\rho_{\mathrm{m}+1}=a \rho_{m}$. An analytical expression for the linear map coefficient $a$ has been obtained, which reveals that $a$ can be interpreted as the effective SEY of the vacuum chamber, $\delta_{\text {eff }}$. This is a convolution of the energy gain after the electron-bunch interaction with the SEY of the vacuum chamber, which also accounts for the electron survival between two bunches. Unfortunately, no analytical expression was found for the function $f\left(\rho_{m}\right)$. Therefore, MEC could not be described as a self-consistent method to describe the electron cloud evolution at a given accelerator. Nonetheless, provided that $a=1$ sets the threshold for the formation of an electron cloud, safe regions in parameter space for RHIC operation are easily determined. The comparison with the values obtained through experimental fits to CSEC simulation results are reasonable.

In the search of an explanation for the sudden changes in the pressure behaviour observed in RHIC, corresponding to so-called first order phase transitions, coupled electron and ion cloud maps have been introduced. The required stability conditions for the stationary electron and ion density have been developed for a given coupling. As an example, a pair of "proof-of-principle" coupled maps have been presented. The analysis using this technique show that hysteresis effects can be observed if an electron cloud coexists with an ion cloud. In these circumstances, the transition from cloud "off" to "on" (and vice-versa) can be abrupt. Furthermore, it is shown that coupled maps predict period doubling and chaotic phenomena. 


\section{PART V}

APPENDIXES 



\section{Appendix A}

\section{Cut-off frequency in a high pass filter}

Together with the capacitor used to protect the amplifier, the impedance in the scope forms an $R C$ circuit similar to a high pass filter. A high pass filter, or differentiator, is shown in Fig. A.1. The frequency cut-off (corresponding to a $3 \mathrm{~dB}$ attenuation) is:

$$
f_{0}=\frac{1}{2 \pi R C},
$$

where $R$ is the line impedance ( $50 \Omega$ ), and the value of $C$ changes depending on the detector. At RHIC, taking into account two typical values of the capacitor,

$$
\begin{aligned}
& f_{0}=32 \mathrm{kHz}, \quad \text { if } \mathrm{C}=100 \mathrm{nF}, \text { and } \\
& f_{0}=320 \mathrm{kHz}, \quad \text { if } \mathrm{C}=10 \mathrm{nF} .
\end{aligned}
$$

Figure A.2 shows the attenuation as a function of the frequency obtained using a Network Analyzer, showing that, in good agreement with Eq. A.1, the $3 \mathrm{~dB}$ level is around 30 and $300 \mathrm{kHz}$, respectively.

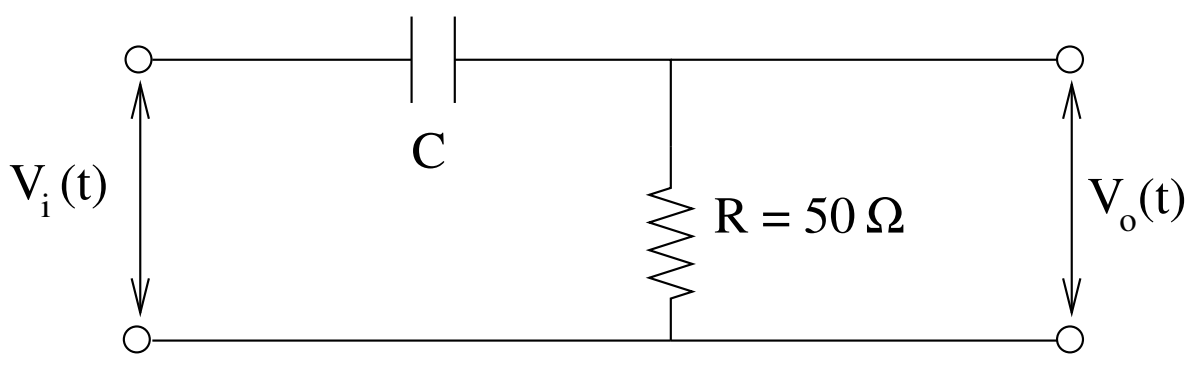

Figure A.1: Sketch of a high pass filter or differentiator.

Analytically, the output voltage as a function of the frequency for this system can be calculated by [111]

$$
V_{0}(f)=\frac{f / f_{0}}{\sqrt{1+\left(f / f_{0}\right)^{2}}} V_{i}(f) .
$$




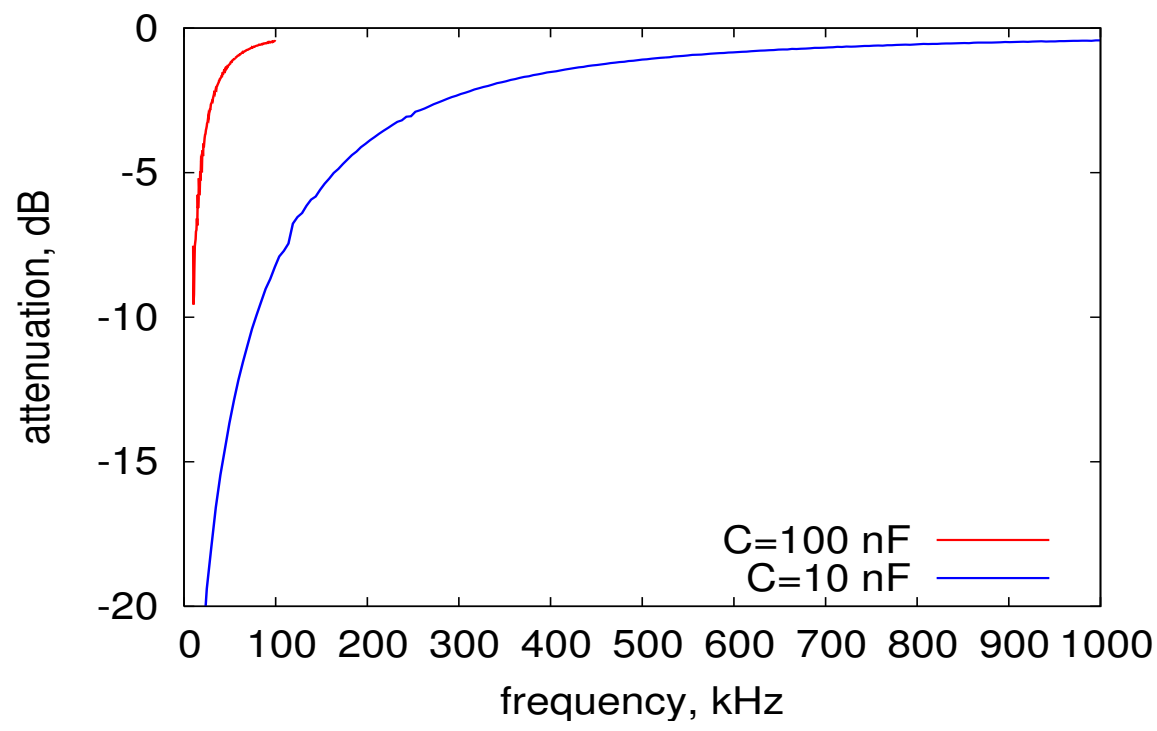

Figure A.2: Measured attenuation using a Network Analyzer of the electron detector electronics. The frequency cutoff decreases from $\approx 300 \mathrm{kHz}$ to $\approx 30 \mathrm{kHz}$ when using a capacitor of 10 or $100 \mathrm{nF}$.

For the RHIC revolution frequency, $f_{\text {rev }}=78 \mathrm{kHz}$, the output voltage is

$$
\begin{array}{ll}
V_{0}=0.95 V_{i}, & \text { if } \mathrm{C}=100 \mathrm{nF}, \text { and } \\
V_{0}=0.24 V_{i}, & \text { if } \mathrm{C}=10 \mathrm{nF},
\end{array}
$$

which shows that for those electron detectors using $C=100 \mathrm{nF}$, it is not needed to account for any attenuation. However, for those electron detectors using $C=10 \mathrm{nF}$, a factor of about 4 is needed due to the system attenuation. For larger frequencies (i.e., bunch spacing $f \approx 10 \mathrm{MHz}$, electron cloud decay $f \approx 5 \mathrm{MHz}$ ), Eq. A.4 shows that $V_{o} \approx V_{i}$. 


\section{Appendix B}

\section{Decay of an electron cloud}

This Annex analyzes the electron density decay after an electron cloud formation. Neglecting the self electric fields, the number of remaining electrons in a monoenergetic jet of $N_{0}$ electrons after $n$ wall collisions is expressed by

$$
N_{n}=N_{0} e^{-n t_{F} / \tau_{d}},
$$

where $\tau_{d}$ refers to the decay time, and $t_{F}$ is the time of flight between two consecutive wall collisions. Assume now the energy of the "jet" is low (around $2 \mathrm{eV}$ ), such that, as shown in Sect. 3.3, the SEY can be interpreted as the elastically reflected probability. The number of electrons after $n$ collisions is then

$$
N_{n}=\delta^{n} N_{0},
$$

where $\delta$ refers to the SEY for low energy electrons. Equating Eqs. B.1 with B.2 leads to

$$
\tau_{d}=\frac{t_{F}}{-\ln \delta} .
$$

To calculate the time of flight $t_{F}$, consider the electron jet only moves in the transverse plane and in the radial direction. In a cylindrical beam pipe with radius $b$, it then follows that

$$
t_{F}=\frac{2 b}{\sqrt{2 E / m_{e}}}
$$

where $E$ is the electron energy, and $m_{e}$ is the electron mass. Using Eq. B.4, the decay time as a function of the low electron energy is

$$
\tau_{d}=\frac{-2 b}{\ln \delta \sqrt{2 E / m_{e}}}=\frac{-b}{c \ln \delta} \sqrt{\frac{2 m_{e} c^{2}}{E}} .
$$

Figure B.1 shows the electron density evolution (red line) for the last four bunch passages in Fig. 3.6, following the CSEC simulation for the case of a bunch population $N_{b}=12 \times$ $10^{10}$ protons, whose input parameters are listed in Table 3.2. The blue line shows the 
average electron energy at wall impact. It is seen that the electron density does not follow the exponential decay expressed by Eq. B.1 along the $\approx 3 \mu$ s of the depicted electron density decay evolution. This is because the electron energy at wall impact decreases by about three orders of magnitude during the same time range. However, one can understand the decay as a combination of two exponential evolutions: a first decay with relatively large electron energies just after the last bunch passage, and as a result of the reminiscences of the electron-bunch interaction, the influence of the "rediffused" electrons (see Section 3.3), space charge effects, etc. The second decay, with very low electron energies (around $0.5 \mathrm{eV}$ ) is understood as a result of the absence of any accelerating electric field. The dashed line shows the fit to the electron density evolution between $t=[6.4,9.5] \mu s$ using the function

$$
f(t)=A_{1} e^{-\left(t-t_{1}\right) / \tau_{1}}+A_{2} e^{-\left(t-t_{2}\right) / \tau_{2}}, \quad \text { with } t_{2}>t_{1}
$$

where $\tau_{1}$ and $\tau_{2}$ are the decay times in the two different regimes described above. The amplitude $A_{1}$ can be considered as the electron density at time $t_{1}$ (the beginning of the first regime), and $A_{2}$ can be considered the electron density at time $t_{2}$ (the beginning of the second regime). The fitting results are listed in Fig. B.1.

For the first decay regime, assuming an average electron energy $E=20 \mathrm{eV}, \delta \approx \delta_{0}=0.6$ (from the CSEC input parameters in Table 3.2), Eq. B.5 results in a decay time $\tau_{d}=88$ ns. For the second decay regime, assuming an average electron energy $E=0.2 \mathrm{eV}, \delta \approx \delta_{0}=0.6$ (from the CSEC input parameters in Table 3.2), Eq. B.5 results in a decay time $\tau_{d}=880 \mathrm{~ns}$. Although the decays times using Eq. B.5 are not accurate, they agree within an order of magnitude with results for $\tau_{1}$ and $\tau_{2}$ in Fig. B.1.

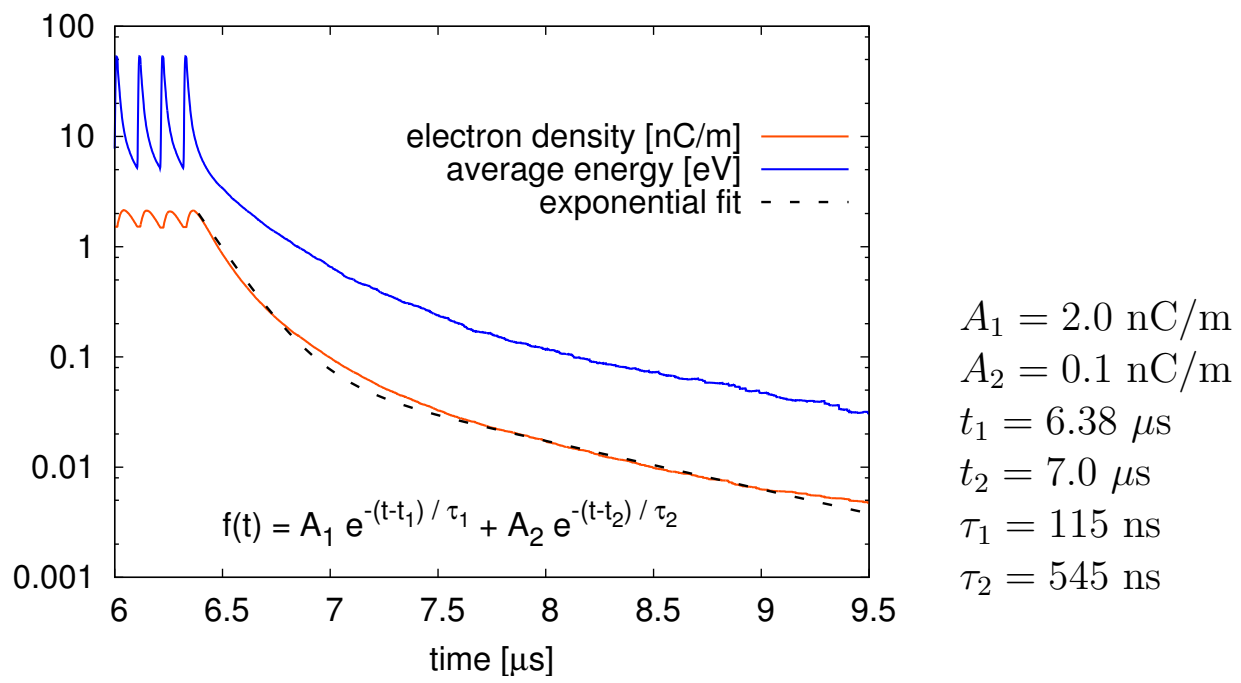

Figure B.1: Electron cloud decay for the CSEC simulation in Fig. 3.6, corresponding to a bunch population of $N_{b}=12 \times 10^{10}$ protons. Depicted is the electron density evolution (red line), and average electron energy at wall impact (blue line). The dashed line shows a fit using the exponential function in Eq. B.6, whose results are listed on the right hand side. 


\section{Appendix $\mathrm{C}$}

\section{Materials and geometry of the RHIC interaction regions}

Figure C.1 shows a sketch of the RHIC IRs geometry specifying the average radius and beam pipe materials. Table C.1 lists the main SEY parameters found in the literature for those materials. With the same beam pipe geometry, the IRs with large Beryllium components (like IR10) should be critical points for electron clouds. For the same beam pipe material, larger beam pipe radius decrease the electron clouds thresholds (like IR12). This is consistent with the observations in RHIC during Run 4 (see Chapter 7 and Ref. [80]).

Table C.1: List of SEY parameters for the different materials in the RHIC IRs. These values refer to raw materials, i.e. without any surface treatments like baking, coating, or electron bombarding.

\begin{tabular}{|l|ccc|}
\hline \hline & Stainless Steel & Aluminum & Beryllium \\
\hline maximum SEY, $\delta_{\max }$ & 2.0 & 2.3 & 2.8 \\
energy at maximum SEY, $E_{\max }$ & 310 & 300 & 250 \\
reference & {$[46,50]$} & {$[48]$} & {$[112]$} \\
\hline
\end{tabular}




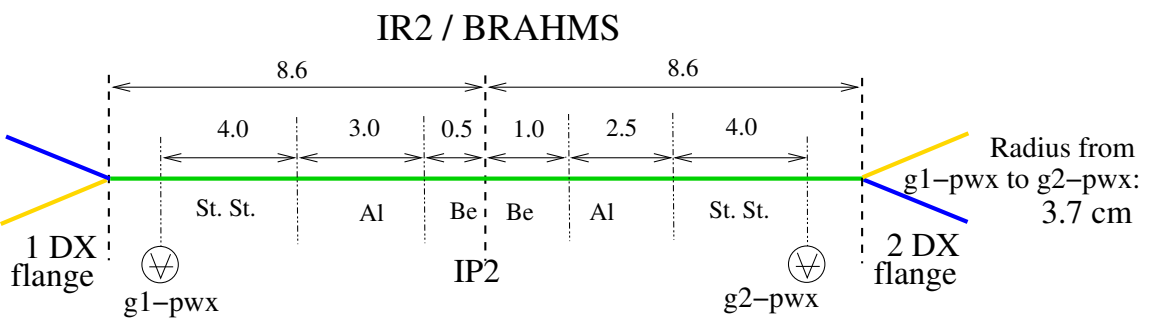

IR4 / RF

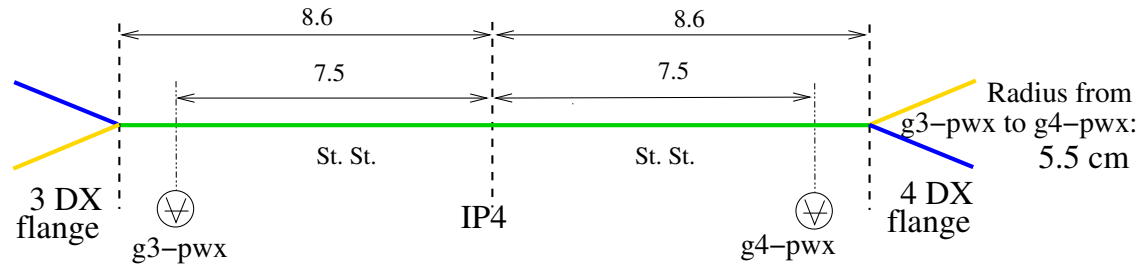

IR6 / STAR

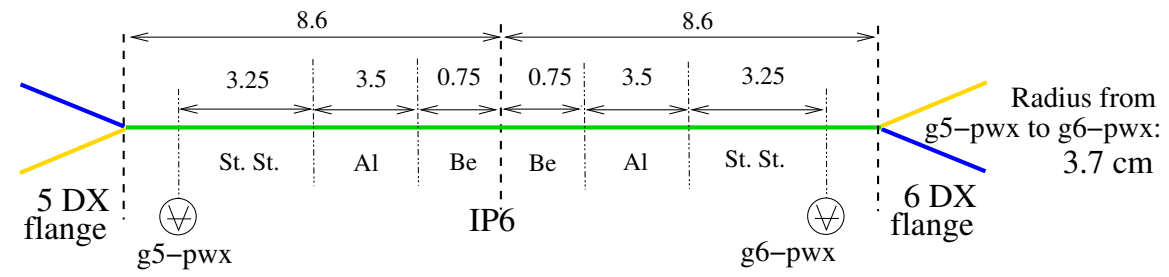

IR8 / PHENIX

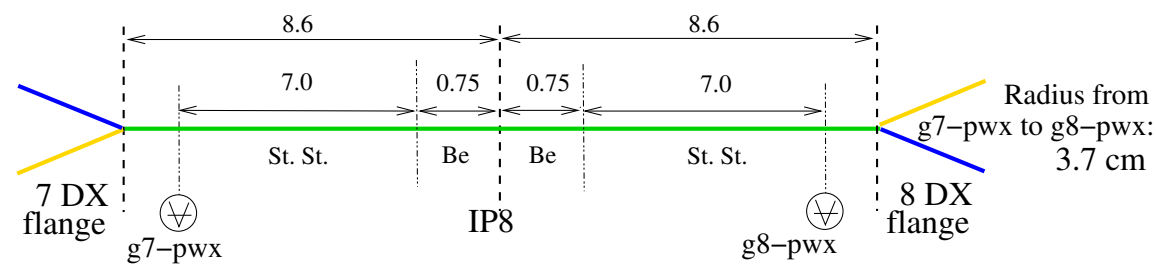

\section{IR10 / PHOBOS}

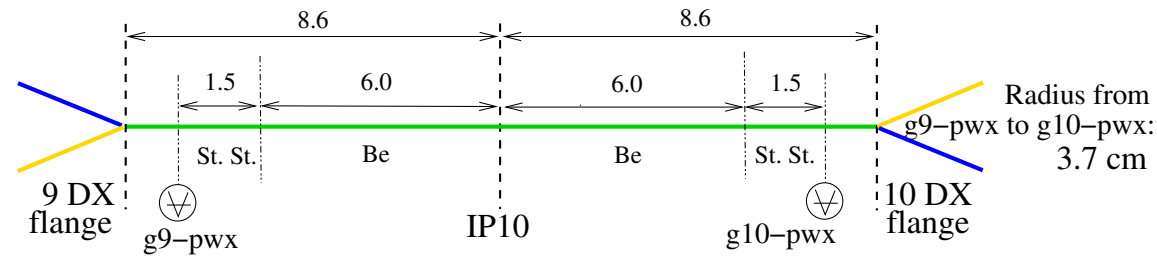

IR12 / H-Jet

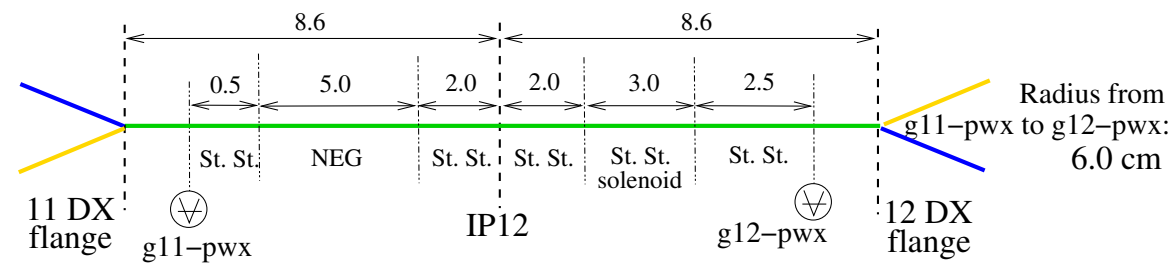

Figure C.1: Geometry of the Interaction Regions during Run 4. Distances are given in [m], and are not to scale. Between the pressure gauge and the DX flange, the beam pipe aperture usually changes by means of cold bore bellows. IR4 geometry is oversimplified due to the large amount of $\mathrm{RF}$ and other beam instrumentation. 


\section{Appendix D}

\section{Linear motion convergence near a fixed point}

The 2 by 2 Jacobian matrix $J$ from Eq. 11.10 at the fixed point can be decomposed as

$$
J=\sqrt{\operatorname{det}(J)} M_{1}
$$

where $M_{1}$ is a unimodular 2 by 2 matrix, with

$$
\operatorname{det}\left(M_{1}\right)=1
$$

Since $J$ is a real matrix, then either $\sqrt{\operatorname{det}(J)}$ and $M_{1}$ are both real, or they are both imaginary, depending on the sign of $\operatorname{det}(J)$. Considering instead the two-step motion

$$
\vec{r}_{\mathrm{n}+2}=J^{2} \vec{r}_{n}
$$

and using the identity

$$
\operatorname{det}\left(J^{2}\right)=\operatorname{det}^{2}(J)
$$

then the decomposition

$$
J^{2}=\operatorname{det}(J) M
$$

now conveniently guarantees that both $M$ and

$$
d \equiv \operatorname{det}(J)
$$

are always real, although $d$ may be negative. The equation of motion is now solved simply as

$$
\vec{r}_{2 n}=d^{n} M^{n} \vec{r}_{0}
$$

The question now is "how does $M^{n}$ behave?"

Next, solve for the two eigenvalues of $M, \lambda_{-}$and $\lambda_{+}$, in the equation

$$
M v=\lambda v
$$


where $v_{-}$and $v_{+}$are the eigenvectors. In other words

$$
\operatorname{det}(M-\lambda I)=0
$$

which is solved by

$$
\lambda_{ \pm}=\frac{1}{2}\left[\operatorname{Tr}(M) \pm \sqrt{\operatorname{Tr}^{2}(M)-4}\right]
$$

For convenience, introduce the notation

$$
T(A) \equiv \frac{1}{2} \operatorname{Tr}(A)
$$

where $A$ is a general matrix, so that the eigenvalues are written more compactly as

$$
\lambda_{ \pm}=T(M) \pm \sqrt{T^{2}(M)-1}
$$

There are now two possibilities - either the two-step motion represented by $M$ is elliptical, or it is hyperbolic.

\section{D.1 Elliptical motion}

The easiest case is if

$$
T^{2}(M)<1
$$

In this case the eigenvalues are both complex, with unit length

$$
\left|\lambda_{ \pm}\right|=1
$$

so that motion is elliptical around the fixed point, spiraling in to converge on the fixed point if

$$
|d|<1
$$

or spiraling out and "escaping" if

$$
|d|>1
$$

\section{D.2 Hyperbolic motion}

Slightly more complicated is the case if

$$
T^{2}(M)>1
$$

In this case the eigenvalues are both real and the motion is hyperbolic around the fixed point. If the initial vector is decomposed as

$$
x_{0}=c_{-} v_{-}+c_{+} v_{+}
$$


then for arbitrary $n$

$$
x_{2 n}=d^{n}\left(c_{-} \lambda_{-}^{n} v_{-}+c_{+} \lambda_{+}^{n} v_{+}\right)
$$

As time goes to infinity $n \rightarrow \infty$ the eigenmode with the larger absolute eigenvalue

$$
\left|\lambda_{1}\right|=|T(M)|+\sqrt{T^{2}(M)-1}
$$

comes to dominate, so that

$$
x_{2 n} \approx c_{1}\left(d \lambda_{1}\right)^{n} v_{1}
$$

This shows that, even though the motion is hyperbolic, it will still converge to the fixed point if (and only if)

$$
\left|d \lambda_{1}\right|<1
$$

Using the substitution

$$
T(M)=\frac{T\left(J^{2}\right)}{d}
$$

this condition is rewritten to explicitly depend only on $J$, as

$$
\left|T\left(J^{2}\right)\right|+\sqrt{T^{2}\left(J^{2}\right)-d^{2}}<1
$$

\section{D.3 Summary}

With the convenient definitions

$$
\begin{aligned}
t & \equiv \frac{\operatorname{Tr}\left(J^{2}\right)}{2} \\
d & \equiv \operatorname{det}(J)
\end{aligned}
$$

Then, if

$$
t^{2}<d^{2}
$$

the motion is elliptical, converging to the fixed point if

$$
d^{2}<1
$$

On the other hand, motion is hyperbolic if

$$
t^{2}>d^{2}
$$

but nonetheless still converges to the fixed point if

$$
|t|+\sqrt{t^{2}-d^{2}}<1
$$

If neither of these pairs of conditions applies, then the motion diverges from the fixed point. 


\section{Bibliography}

[1] P. Farnsworth. J. Franklin Inst., 218(4):411-444, October 1934.

[2] F. Zimmermann and G. Rumolo. Electron cloud build up in machines with short bunches. ICFA Beam Dynamics Newsletters, 33, April 2004.

[3] G.I. Dimov G.I. Budker and V.G. Dudnikov. In Proceedings of the International Symposium on Electron and Positron Storage Rings, pages VIII-6-1. Universitaires de France, Orsay, 1966.

[4] O. Gröbner. Bunch induced multipacting. In Proceedings of the 10th International Conference on High Energy Accelerators, page 277. USSR Academy of Science, Protvino, 1977.

[5] G. Rumolo and F. Zimmermann, editors. Proceedings of ECLOUD'02, Mini-Workshop on Electron-Cloud Simulations for Proton and Positron Beams. CERN, June 2002.

[6] R. Kirby et al. Instrumental effects in secondary electron yield and energy distribution measurement. In Proceedings of ECLOUD'04, 2004.

[7] H. Hahn (Ed.). RHIC design manual, October 2000, available at http://www.agsrhichome.bnl.gov/NT-share/rhicdm/00_toc1i.htm.

[8] W. Fischer, J.M. Brennan, M. Blaskiewicz, and T. Satogata. Electron cloud measurements and simulations for the Brookhaven Relativistic Heavy Ion Collider. Phys. Rev. $S T-A B, 5(124401), 2002$.

[9] http://www.bnl.gov/rhic/star.htm.

[10] http://www.phenix.bnl.gov/phenix/www/intro/index.html.

[11] http://www.phobos.bnl.gov/overview.htm.

[12] http://www4.rcf.bnl.gov/brahms/www/overview.html.

[13] http://www.rhic.bnl.gov/pp2pp/.

[14] M. Conti and W.W. McKay. An introduction to the Physics of Particle Accelerators. World Scientific, 1991. 
[15] D.A. Edwards and M.J. Syphers. An introduction to the physics of high energy accelerators. John Wiley \& Sons Inc., New York, 1993.

[16] H. Wiedemann. Particle Accelerator Physics I. Springer-Verlag, New York, 1998.

[17] Soren Pape Moller. Beam-residual gas interactions. In S. Turner, editor, CAS on Vacuum Technology. CERN, pp. 155, Geneva, 1999.

[18] M.J. Rhoades-Brown and M. Harrison. Vacuum requirements for RHIC. Technical report, BNL, AD-RHIC 106, December 1991.

[19] H.C. Hseuh et al. Comissioning of the RHIC vacuum systems. In Proceedings of PAC'99, 1999.

[20] W. Fischer, R. Connolly, S. Tepikian, J. van Zeijts, and K. Zeno. Intra-beam scattering measurement at RHIC. In Proceedings of EPAC'02, 2002.

[21] M. Pivi. Beam induced electron multipacting in the LHC. PhD thesis, Univ. of Torino., 2000.

[22] U. Iriso, F. Caspers, J.-M. Laurent, and A. Mostacci. Traveling wave resonant ring for electron cloud studies. Phys. Rev. ST-AB, 7(073501), 2004.

[23] L. Wang, M. Blaskiewicz, J. Wei, W.T. Weng, K. Ohmi, and R.J. Macek. Mechanism of electron multipacting with a long-bunch proton beam. Phys. Rev., E70(03651), 2004.

[24] M. Blaskiewicz, M.A. Furman, M. Pivi, and R.J. Macek. Electron cloud instabilities in the Proton Storage Ring and Spallation Neutron Source. PRST-AB, 6(014203), 2003.

[25] J. Wei and R.J. Macek. Electron cloud effects in high intensity proton accelerators. In Proceedings of ECLOUD'02 Workshop, Geneva, April 2002.

[26] O. Gröbner. Beam inducing multipacting. In pp. 3589, Proceedings of PAC'97, Vancouver, 1997.

[27] K. Harkay and R.A. Rosenberg. Properties of the electron cloud in a high energy positron and electron storage ring. PRST-AB, 6(034402), 2003.

[28] G. Rumolo, E. Benedetto, U. Iriso, and F. Zimmermann. Single bunch instabilities induced by electron cloud in short positron/proton/ion bunches. ICFA Beam Dynamics Newsletters, 33, April 2004.

[29] J. Wei, M. Blaskiewicz, W. Fischer, H.C. Hseuh, U. Iriso, L. Wang, and S.Y. Zhang. Electron cloud effects - observations, mitigation measures, and challenges in RHIC and SNS. In Proceedings of CARE Workshop, Geneva, November 2004.

[30] P. Thieberger, A.L. Hanson, D.B. Steski, V. Zajic, S.Y. Zhang, and H.Ludewig. Secondary-electron yields and their dependence on the angle of incidence on stainlesssteel surface for three energetic ion beams. Phys. Rev. A, 61(042901), March 2000. 
[31] S. Berg. Energy gain in an electron cloud during the passage of a bunch. Technical Report 97, CERN LHC Project Note, July 1997.

[32] M. Furman. Electron-cloud build-up in hadron machines. ICFA Beam Dynamics Newsletters, 33, April 2004.

[33] W. Fischer et al. Vacuum pressure rise with intense ion beams in RHIC. In Proceedings of EPAC'02, June 2002.

[34] H.C. Hseuh, L. Smart, and S.Y. Zhang. Analysis of beam induced pressure increases in RHIC warm vacuum sections. In Proceedings of EPAC'02, June 2002.

[35] S.Y. Zhang. RHIC vacuum pressure bump. Technical report, BNL, C-AD 67, 2002.

[36] M. Furman. Incoherent effects driven by the electron cloud. In Proceedings of PAC'99, 1999.

[37] K. Ohmi. Head-Tail Instability Caused by Electron Clouds in Positron Storage Rings . Phys. Rev. Lett., 85, 2000.

[38] G. Rumolo, F. Zimmermann, H. Fukuma, and K. Ohmi. Electron cloud studies for KEKb. In Proceedings of PAC 2001, 2001.

[39] G. Arduini, K. Cornelis, G. Ferioli, L. Jensen, and F. Zimmermann. Transverse instabilities of the LHC proton beam in the SPS. In Proceedings of EPAC 2000, 2000 .

[40] G. Arduini, K. Cornelis, W. Hofle, G. Rumolo, and F. Zimmermann. Transverse behaviour of the LHC proton beam in the SPS: an update. In Proceedings of PAC 2001, 2001.

[41] J.D. Jackson. Classical electrodynamics, chapter 11. John Wiley \& Sons Inc., 3rd edition edition, New York, 1998.

[42] L.D. Landau and E.M. Lifshitz. Mechanics, course of theoretical physics, chapter 7, pp. 132. Buterworth Heinemann, 3rd edition edition.

[43] G. Rumolo and F. Zimmermann. Electron-cloud simulations: build up and related effects. In Proceedings of ECLOUD'02, April 2002.

[44] H. Bruining. Physics and Applications of Secondary Electron Emission. Pergamon Press, McGraw-Hill Book Co., New York, 1954.

[45] P.A. Redhead, J.P. Hobson, and E.V. Kornelsen. The Physical Basis of Ultrahigh Vacuum. Chapman and Hall, London, 1968. Reprinted by the AIP in 1993 as part of the American Vacuum Society Classics series. 
[46] M. Furman and M. Pivi. Microscopic probabilistic model for the simulation of secondary electron emission. PRST-AB, 5(12404), December 2002.

[47] R. Cimino, I. Collins, M. Furman, M. Pivi, G. Rumolo, and F. Zimmermann. Can low energy electrons affect high energy accelerators? Phys. Rev. Lett., 93(014801), 2004.

[48] R. Kirby and F.K. King. Secondary electron emission from PEP-II accelerator materials. Nucl. Inst. and Meth. in Phys. Research, A(469), 2001.

[49] H. Seiler. Secondary electron emission in the scanning electron microscope. J. Appl. Phys, 54(11), 1983.

[50] V. Baglin, J. Bojko, O. Gröbner, B. Henrist, N. Hilleret, C. Scheuerlein, and M. Taborelli. The Secondary Electron Yield of technical materials and its variation with surface treatments. Technical Report LHC Project Report 433, CERN, Sept. 2000.

[51] Relevant publications about surface properties are updated at http://at-divvac.web.cern.ch/atdivvac/vacpages/ps/phys\& tech/phys/ecloud/sey/sey_paper.html.

[52] G. Bellodi. Code comparisons and benchmarking with different SEY models in electron cloud build-up simulations. In Proceedings of CARE'04, November 2004.

[53] S. Heifets. Electron cloud at high beam currents. In Proceedings of ECLOUD'02, Geneva, 2002.

[54] F. Zimmermann, G. Bellodi, G. Rumolo, M. Blaskiewicz, M.F. Furman, M. Pivi, L. Wang, and K. Ohmi et al. Review and comparison of simulation codes modeling electron-cloud build-up and instabilities. In Proceedings of EPAC'04, Lucern, 2004.

[55] M. Furman. Issues in the formation and dissipation of the electron cloud. In Proceedings of Pressure Rise Workshop, BNL, December, 2003.

[56] R. Macek et al. Recent experimental studies of the electron cloud at the Los Alamos PSR. In KEK Workshop on Two Stream Instabilities. KEK, September 2001.

[57] R.A. Rosenberg and K.C. Harkay. A rudimentary electron energy analyzer for accelerator diagnostics. NIM, A(453):507-513, 2000.

[58] U. Iriso and J-M. Laurent. Particle collectors for electron cloud studies. Technical Report TN03-05, LHC-VAC CERN, February 2003.

[59] Z.Y. Guo et al. Experiment to measure the electron cloud at BEPC. In Proceedings of $P A C^{\prime} 01,2001$.

[60] http://www.cadops.bnl.gov/people/gullotta/ed-config.html.

[61] http://www.orcadpcb.com/pspice. 
[62] E.R. Beadle and V.J. Castillo, editors. Accelerator instrumentation, Upton - NY, 1989. R.E. Shafer, "Beam Position Monitoring", pp.26.

[63] C. Montag and J. Kewisch. Commisisioning of a first-order matched transition jump at RHIC. PRST-AB, 7(011001), 2004.

[64] P. He, H.C. Hseuh, D. Gassner, J. Gullotta, D. Trbojevic, and S.Y. Zhang. Calibration of RHIC electron detectors. In Proceedings of PAC'03, May, 2003.

[65] U. Iriso et al. Electron cloud observations at RHIC during Run-3 (2002/2003). Technical report, BNL, C-AD, 129, 2003.

[66] S. Turner, editor. CAS on Vacuum Technology. CERN, Geneva, 1999.

[67] G. Stupakov. Photoelectrons and multipacting in the LHC: Electron cloud build-up. Technical Report 141, CERN, LHC Project Report, October 1997.

[68] H. Hseuh. Private communications, 2004.

[69] J. Gomez-Goni and A.G. Mathewson. Temperature dependence of the electron induced gas desorption yields on stainless steel, Copper and Aluminum. J. Vac. Sci. Technol., A(15), Nov./Dec 1997.

[70] F. Billard, N. Hilleret, and G. Vorlaufer. Some results on the electron induced desorption yield of OFHC Copper. Technical Report LHC/VAC/FB/NH/GV00-32, CERN, 2000 .

[71] K. Kanazawa and H. Hisamatsu. Presentation at BNL, October 2004.

[72] Y. Suetsugu. Observation and simulation of the nonlinear dependence of vacuum pressures on the positron beam current at the KEKb. In Proceedings of PAC'01, 2001.

[73] A. Zelenski, A. Bravar, D. Grahama, W. Haeberli, S. Kokhanovski, Y. Makdisi, G. Mahler, A. Nass, J. Ritter, T. Wise, and V. Zubets. Absolute polarized H-Jet polarimeter development for RHIC. In PST 2003 Workshop on polarized sources and targets, September 2003.

[74] N. Hilleret, B. Henrist, C. Scheuerlein, M. Taborelli, and G. Vorlaufer. The variation of the Secondary Electron Yield and of the desorption yield of Copper under electron bombardment: origin and impact on the conditioning of the LHC. In Proceedings of EPAC'02, page 2553, 2002.

[75] V. Baglin et al. Measurements at epa of vaccuum and electron-cloud related effects. In Proceedings of the $11^{\text {th }}$ Chamonix Workshop, Jannuary 2001.

[76] J. Wei, U. Iriso, M. Bai, M. Blaskiewicz, P. Cameron, R. Connolly, A. Della Penna, W. Fischer H. Huang, R. Lee, R. Michnoff, V. Ptitsin, T. Roser, T. Satogata, S. Tepikian, L. Wang, and S.Y. Zhang. Observation of electron-ion effects at RHIC transition. In Proceedings of $P A C^{\prime} 05,2005$. 
[77] M. Blaskiewicz. Private communications, 2005.

[78] G. Rumolo and W. Fischer. Observations on background in PHOBOS and related electron cloud simulations. Technical report, BNL, C-AD, 146, March 2004.

[79] S. Peggs and U. Iriso. Electron cloud phase transitions. Technical report, BNL, C-AD, 147, April 2004.

[80] W. Fischer and U. Iriso. Transition pressure rise in RHIC during Run-4. Technical report, BNL, C-AD, 184, December 2004.

[81] W. Fischer, M. Blaskiewicz, P. He, H. Huang, H.C. Hseuh, U. Iriso, G. Rumolo, L. Smart, D. Trbojevic, and S.Y. Zhang. Electron clouds and vacuum pressure rise in RHIC. In Proceedings of ECLOUD'04, 2004.

[82] S.Y. Zhang et al. RHIC pressure rise and electron cloud. In Proceedings of the PAC'03, May 2003.

[83] S.Y. Zhang. Ion Desorption at RHIC. Technical report, BNL/CAD/182, and Proceedings of the ICFA Workshop on High Intensity and High Brightness Beams, Bensheim, Germany, 2004.

[84] E. Mahner, I. Efthymiopoulos, J. Hansen, E. Page, and H. Vincke. Beam-loss induced pressure rise of LHC collimator materials irradiated with $158 \mathrm{GeV} / \mathrm{u} \mathrm{In}^{49+}$ ions at the CERN SPS. PRST-AB, 2005.

[85] U. Iriso, P. He, H.C. Hseuh, H. Huang, V. Ptitsyn, L. Smart, P. Thieberger, and D. Trbojevic. Interpretation of desorption measurements for high energy beams at RHIC. Technical report, BNL/CAD/178, September 2004.

[86] J. M. Jiménez. Measurement of Electron Cloud Effects in SPS. Beam Dynamics Newsletters, 33, April 2004.

[87] U. Iriso and S. Peggs. Maps for Electron Clouds. PRST-AB, 8:024403, January 2005.

[88] W. Fischer. Private communications, 2004.

[89] W. Fischer and U. Iriso. Bunch patterns and pressure rise in RHIC. In Proceedings of EPAC'04, Lucern, June 2004.

[90] W. Fischer and U. Iriso. Bunch patterns and pressure rise in RHIC. Technical report, BNL, C-AD/AP/118, October 2003.

[91] Y. Cai, M. Pivi, and M.A. Furman. Buildup of electron cloud in the PEP-II particle accelerator in the presence of a solenoid field and with different bunch pattern. PRST$A B$, 7:024402, February 2004. 
[92] L. Wang, D. Raparia, J. Wei, and S.Y. Zhang. Mechanism of electron cloud clearing in the accumulator ring of the SNS. PRST-AB, 7:034401, March 2004.

[93] F. Le Pimpec, K. King, R.E. Kirby, and M. Pivi. Properties of TiN and TiZrV thin film as a remedy against electron cloud. NIM - A, A, p.187-199, 2005.

[94] C. Benvenuti. Molecular surface pumping: the getter pumps. In S. Turner, editor, CAS on Vacuum Technology. CERN, pp. 43, Geneva, 1999.

[95] C. Benvenuti et al. Vacuum properties of TiZrV non-evaporable getter films for LHC vacuum system. Vacuum (UK), 60, no.1-2, p.57-65, Jan.-Feb. 2001.

[96] http://www.saesgetters.com.

[97] P. Costa Pinto et al. NEG coating of the non-standard LSS vacuum chambers. In TS Workshop - Site d'Archamps. CERN, May 2005.

[98] H.C. Hseuh. Private communications, September 2004.

[99] K. Ohmi. Beam-Photoelectron Interactions in Positron Storage Rings. Phys. Rev. Lett., 75, April 1995.

[100] R.M. May. Simple mathematical models with very complicated dynamics. Nature, 261:459, June 1976.

[101] G. Rumolo and F. Zimmeramnn. Practical user guide for ECLOUD. Technical Report SL-Note-2002-016-AP, CERN, May 2002.

[102] J.N. Lawton M.P. Hassell and R.M. May. Patterns of dynamical behaviour in singlespecies populations. Journal of Animal Ecology, 45:471-486, 1976.

[103] J. Bascompte and R. Sole. Spatially induced bifurcations in single-species population dynamics. Journal of Animal Ecology, 63:256-264, 1994.

[104] U. Iriso, M. Blaskiewicz, A. Drees, W. Fischer, S. Peggs, and D. Trbojevic. Electron cloud and pressure rise simulations for RHIC. In Proceedings of PAC'03, Portland, May 2003.

[105] W. Fischer et al. Luminosity increases in Gold-Gold operation in RHIC. In Proceedings of EPAC'04, Lucern, June 2004.

[106] U. Iriso et al. Analysis of the electron cloud in RHIC. In Proceedings of EPAC'04, Lucern.

[107] W. Fischer, U. Iriso, and E. Mustafin. Electron cloud driven vacuum instability. In AIP Conference Proceedings 773, 33rd ICFA Advanced Beam Dynamics Workshop on High Intensity and High Brightness Hadron Beams, pages 204-206, 2005. 
[108] G. Rumolo and F. Zimmermann. Interplay of ionization and sputtering with the electron cloud. Technical report, CERN, 2001.

[109] O. Gröbner. Electron cloud driven vacuum instability. In Proc. of CARE HHH-2004 Workshop .

[110] P. Thieberger, U. Iriso, and S. Peggs. Electron cloud maps and first order phase transition at RHIC. to be published, 2005.

[111] G.M. Jenkins and D.G. Watts. Spectral analysis and its aplications. Holden-Day Inc., 1968. Ch. 2.

[112] N. Hilleret and B. Henrist. Secondary Emission Yield of Beryllium. Technical report, CERN LHC/VAC/TN-00-025, 2000. 


\section{Publications}

\section{Refereed publications:}

- U. Iriso and W. Fischer. Electron induced molecular desorption from electron clouds at the Relativistic Heavy Ion Collider. Phys. Rev. ST Accel. Beams 8, 113201 (2005).

- U. Iriso and S. Peggs. Maps for electron clouds. Phys. Rev. ST Accel. Beams 8, 024403 (2005).

- U. Iriso, F. Caspers, J-M. Laurent, and A. Mostacci. Traveling wave resonant ring for electron cloud studies. Phys. Rev. ST Accel. Beams 7, 073501 (2004).

- U. Iriso, and S. Peggs. Coupled maps for electron and ion clouds. Submitted to Phys. Rev. ST Accel. Beams, (2005).

- P. Thieberger, U. Iriso, and S. Peggs. Maps, electron clouds, and first order phase transitions in the Relativistic Heavy Ion Collider. Submitted to Phys. Rev. ST Accel. Beams, (2005).

\section{Non-refereed publications:}

- J. Wei, U. Iriso, et al. Observation of Electron-Ion effects at RHIC Transition. Proceedings of PAC 2005, Oak Ridge (USA), May 2005.

- S.Y. Zhang et al. Beam induced pressure rise in RHIC. Proceedings of PAC 2005, Oak Ridge (USA), May 2005.

- F. Pilat et al. Operation and performance of RHIC as a Cu-Cu collider. Proceedings of PAC 2005, Oak Ridge (USA), May 2005.

- M. Bai et al. Polarized proton collisions in RHIC. M. Bai et al. Proceedings of PAC 2005, Oak Ridge (USA), May 2005.

- W. Fischer, U. Iriso, and E. Mustafin. Electron cloud driven vacuum instability. Proceedings of Beam Dynamics Workshop on High Intensity and High Brightness Beams, Bensheim, Germany, October 2004.

- U. Iriso. Electron cloud observations at RHIC. ICFA Beam Dynamics Newsletter, N\# 33: Two-stream effects in accelerators, 2004. 
- G. Rumolo, E. Benedetto, U. Iriso, and F. Zimmermann. Single bunch instabilities induced by electron cloud in short positron/proton/ion bunches. ICFA Beam Dynamics Newsletter, N\# 33: Two-stream effects in accelerators, 2004.

- U. Iriso et al. Analysis of electron cloud at RHIC. Proceedings of EPAC 04, Lucerne, Switzerland. July 2004.

- U. Iriso and S. Peggs. Maps for fast electron cloud simulations at RHIC. Proceedings of EPAC 04, Lucerne, Switzerland. July 2004.

- U. Iriso and S. Peggs. Using maps for exploration of electron cloud parameter space. Proceedings of ECLOUD 04, Napa Valley, USA. April 2004.

- W. Fischer and U. Iriso. Bunch patterns and pressure rise at RHIC. Proceedings of EPAC 04, Lucerne, Switzerland. MOPLT164. July 2004.

- U. Iriso et al. Electron Detectors for Vacuum Pressure Rise Diagnostics at RHIC. Proceedings of PAC03, Portland. May 2003.

- U. Iriso et al. Electron Cloud and Pressure Rise Simulations for RHIC. Proceedings of PAC03, Oregon, May 2003.

- S.Y. Zhang et al. RHIC Pressure Rise and Electron Cloud, S.Y. Zhang. Invited talk at PAC03, Oregon. Proceedings of PAC03. May 2003.

- U. Iriso, F. Caspers, A. Mostacci. Evaluation of the Horizontal to Vertical Transverse Impedance Ratio for LHC Beam Screen Using a 2D Electrostatic Code. Proceedings of PAC03, Oregon. May 2003.

- A. Mostacci, U. Iriso, F. Caspers, L. Vos. Bench Measurements of Low Frequency Transverse Impedance. Proceedings of PAC03, Oregon. May 2003.

- U.Iriso, J.-M. Laurent, F. Caspers and A. Mostacci. Ring Resonator test bench for electron cloud studies. Proceeding of EPAC'02, Paris, June 2002.

- U.Iriso, J.-M. Laurent, F.Caspers and A.Mostacci. RF test benches for electron cloud studies. Talk given in ECLOUD'02, Mini-Workshop on Electron-Cloud Simulations for Proton and Positron Beams. CERN, April 15-18, 2002. 


\section{Acknowledgments}

I wish to thank Dr. Steve Peggs, who gave me the opportunity to join BNL and carry out this Phd thesis. His fanciful knowledge of Physics and his enthusiasm have been a great motivation during this time.

I must acknowledge Dr. Joan Esteve for his patience and support, which ultimately made possible the presentation of this work at the University of Barcelona.

I am indebted to Dr. Angelika Drees, not only for providing precious help in any Accelerator Physics topic but also for her support and friendship. Without her kind hosting, it wouldn't have been possible to land at BNL.

I have to thank Mike Blaskiewicz for sharing his fascination for Physics and because he basically helped me a hell of a lot. He originally wrote the code CSEC, and was always ready to help me out with it.

I am grateful to Dr. Wolfram Fischer because I learned a lot from his vast knowledge and his efficient way of tackling problems. Certainly, attention to detail finally pays off.

I am indebted to Dr. Rogelio Tomás, and so is this thesis. His sharing of computing knowledge and fruitful discussions became a source of learning and laughing.

I wish to thank S.Y. Zhang for pointing out many interesting phenomena occurring at RHIC, H.C. Hseuh for his endless patience answering any question I could have, to L. Smart and $\mathrm{P}$. He for their help in the vacuum business. The reliability of the hardware for the electron detector is a result of the great work of D. Gassner and J. Gullotta, while the electron detector's software owes its efficiency to V. Ponnaiyan and R.C. Lee. I am very grateful to T. Satogata for his remarkable patience with all my computing problems.

I would like to show my gratitude to T. Roser, D. Trbojevic, M. Bai, F. Pilat, H. Huang, V. Ptitsyn, all the C-AD department, all the Administrative staff, and all the operations group for their valuable support and help.

This thesis owes a lot to the discussions I had with external collaborations from CERN (F. Zimmermann, G. Arduini, F. Ruggiero, J.M. Jiménez, A. Rossi, V. Baglin...), from LBNL (M. Furman), from SLAC (M. Pivi, F. Le Pimpec), from RAL (G. Bellodi). Among them, I am obliged to remark the kindness, knowledge, and tirelessly support of J.-M. Laurent (CERN). During this time, I was very lucky to account with G. Rumolo (CERN, GSI, CELLS, etc) as a friend and collaborator.

I have to thank my office mates Javier, Ray, Rama, and Jay for the useful comments, doubts solved, and great times shared.

A significant number of people made my stay in BNL and New York a great time. Sanjee was one of the first in appropriately filling my leisure time, Keith provided nice 
gastronomical discussions and tours around New York, and Nick was a constant source of interesting (language, history, politics...) discussions. This thesis would have not been possible without their help, their language assessments, the caffeine doses from KSS and the subsequent excursions to the theater. I must thank the manhattaners, Paloma, Angel, Fernanda, Bittor, and Nico for the funny nights around the city.

My CERN colleagues for the nice time I spent in Geneva, Arturo, Jeremie, Fabienne, Pilar, Eric, Laurent, Carlos B., Julien, Cristina, Toni, Marco C., Marco L., Luis, Juan, Mario, Mirko, Jan, Federico, Stefano R., Stefano P., Bobo, Alberto, Eva, Laura...

Haig d'agrair als companys de facultat els divertits inicis que vam compartir a la facultat. Al Ramó i la Susanna, i a l'ordinador de la Susanna per l'ajuda prestada.

Seguidament, he de reconèixer a tota la tropa ametlletana l'amistat que m'han proporcionat durant 30 anys, que es diu ràpid i en són molts. I clar, al Litus i al Quim, perquè han passat pocs dies en aquests anys sense que el seu record m'hagi fet somriure.

A mi família al completo, porque es cierto eso de que todo esto no hubiese sido posible sin su total apoyo.

I a l'Esther, per estar amb mi tots aquests anys malgrat la maleïda distància. Sé que mai podré agrair-li com es mereix. 